\title{
ASSESSMENT OF MICROBIAL BIODEGRADATION OF MIXED SOIL CONTAMINANTS AT THE SANTA SUSANA FIELD LABORATORY USING TRFLP, qPCR, AND CULTURING
}

\author{
A Thesis \\ Presented to \\ the Faculty of California Polytechnic State University, \\ San Luis Obispo \\ In Partial Fulfillment \\ of the Requirements for the Degree
}

Master of Science in Engineering with Specializations in Biochemical Engineering

$$
\text { By }
$$

Kenneth William Croyle

August 2014 
(C) 2014

Kenneth William Croyle

ALL RIGHTS RESERVED 


\section{COMMITTEE MEMBERSHIP}

TITLE:

AUTHOR:

DATE SUBMITTED:

COMMITTEE CHAIR:

COMMITTEE MEMBER:
Assessment of Microbial Biodegradation of Mixed Soil Contaminants at the Santa Susan Field Laboratory Using TRFLP, qPCR, and Culturing

Kenny William Croyle
Yarrow Nelson, Ph.D.

Professor of Environmental Engineering
Chris Kitts, Ph.D.

Professor of Biology

Nirupam Pal, Ph.D.

Professor of Environmental Engineering 


\begin{abstract}
Assessment of Microbial Biodegradation of Mixed Soil Contaminants at the Santa Susan Field Laboratory Using TRFLP, qPCR, and Culturing Kenny William Croyle
\end{abstract}

The potential for biodegradation of contaminants in soil was assessed using an array of molecular methods, including terminal restriction fragment length polymorphism (TRFLP), quantitative polymerase chain reaction (qPCR), and traditional culturing techniques combined with sequencing of the 16S or ITS regions of the cultured bacteria and fungi. Soil was collected from the Santa Susana Field Laboratory (SSFL), which was the site of numerous liquid-propulsion rocket engine tests as well as nuclear energy research and development, which led to contamination of the soil with a wide variety of constituents. The contaminants of interest (COIs) at this site include polychlorinated biphenyls (PCBs), dioxins, polycyclic aromatic hydrocarbons (PAHs) and non-PAH petroleum hydrocarbons (PHCs). Various metals, most notably mercury and silver, are also present on the site. The purpose of this study was to determine if biodegradation is contributing to natural attenuation of contaminants in the soil, what organisms are likely causing biodegradation, and what rate(s) can be expected in the future. A literature review was conducted to investigate the chemical properties of theses COIs, their toxicity, and abiotic and biotic degradation. This research concluded that these COIs can be biodegraded if the right bacteria and/or fungi are present and active in the soil in sufficient numbers under the right conditions. Many known biodegraders of the COIs 
were identified in the literature review along with the most common pathways of biodegradation and degradation rates observed in field and laboratory studies.

Soil was collected from 30 sample locations, with 3 sets of 10 samples containing high concentrations of one COI but low concentration of the others. PHCs and PAHs were found to be largely co-located, so 10 samples were selected for both of them. The remaining 20 samples were split evenly between PCBs and dioxins. DNA was extracted directly from all 30 soil samples and amplified using PCR for TRFLP analyses. Two soil samples were sent to Microbial Insights® for qPCR analysis. This analysis included 18 gene targets for the degradation of PHCs and PAHs, as well as the target gene for Dehalococcoides (an anaerobic dechlorinating bacteria). For each culturing a model chemical was selected to represent each COI and added to Bushnell-Haas agar plates containing no added carbon source other than the model compounds. The model chemicals were No. 2 diesel fuel for PHCs, naphthalene for PAHs, PCB \#1 (monochloro) for PCBs, and dibenzofuran for dioxin. These plates were used to screen for biodegrading bacteria and fungi for each COI. Once cultured, 16S and ITS sequencing were used to identify these potential COI degraders and determine what TRFLP peak they would produce. The identity of isolated organisms was compared to information from the literature to assess the likelihood of COI biodegradation at SSFL.

From the culturing experiments, 45 organisms were isolated, sequenced, and identified. The 45 included 14 unique bacteria and seven unique fungi. Of these, 10 different bacterial species and 5 different fungal species have been reported as COI biodegraders or belong to genera that contain reported COI biodegraders. TRFLP analysis revealed that the soil type has more effect on the microbial population than the 
presence of any of the COIs. There were no specific peaks that were significantly correlated to any specific COI. The peak distributions were fairly even, indicating a large amount of biodiversity in the microbial populations of the soil samples. The qPCR analysis revealed that SSFL soils contain significant populations of microbes that can degrade PHCs aerobically. Anaerobic PHC, anaerobic PAH, and aerobic PAH targets were not detected. A small amount of Dehalococcoides was detected in one of the samples.

Collectively this study suggests that microbes present in SSFL soils are capable of biodegrading PHCs, and the genes for such biodegradation are actively being expressed. With the exception of a small population of Dehalococcoides, bacteria associated with the biodegradation of PAHs, PCBs, and/or dioxins were not detected. However, several strains of fungi were identified which have been reported to mediate cometabolic biodegradation of these compounds. Since these fungi do not require anaerobic conditions, they are more likely to contribute to natural attenuation than bacterial reductive dechlorination. Laboratory microcosm experiments are suggested for estimating rates of biodegradation at SSFL under natural attenuation conditions. Bioaugmentation and/or biostimulation methods should also be investigated in addition of natural attenuation. These microcosm experiments are currently underway in a companion study at Cal Poly by graduate student Mackenzie Billings. 


\section{ACKNOWLEDGMENTS}

I would like to thank my advisor Dr. Yarrow Nelson for his guidance, support, and assistance throughout this project. I would like to thank Dr. Chris Kitts for his help and advice regarding experimental design and TRFLP analysis. Thank you to Alice Hamrick for her help and guidance regarding TRFLP, DNA sequencing, and culturing techniques. I'd also like to thank Keegan Roberts and the staff at CDM Smith for their constant support and guidance. A special thanks to DOE for funding this study. I'd like to thank my fellow grad students Mackenzie Billings and Matt Poltorak for their help with the literature review and numerous other aspects of this project. I'd also like to acknowledge the contributions of my undergraduate lab assistants Adam Caughey and Krista Pratico for their assistance with the literature review and culturing experiments. 


\section{TABLE OF CONTENTS}

List of Tables ................................................................................................. xii

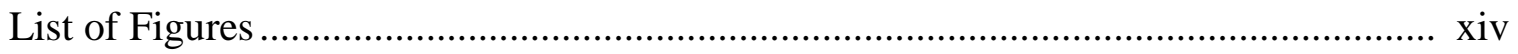

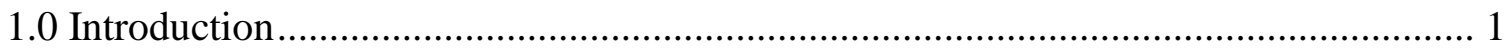

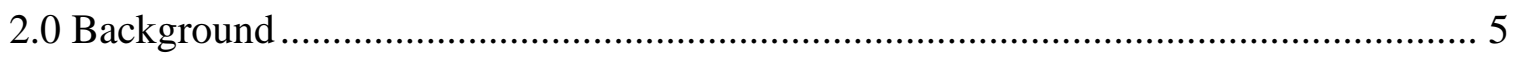

2.1 SSFL Site Background and Project Origin...............................................5

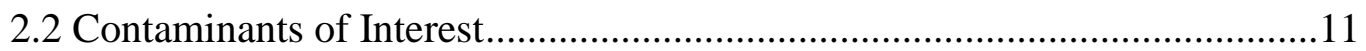

2.2.1 Petroleum Hydrocarbons (PHCs) ..................................................... 12

Physical Properties .............................................................. 12

Petroleum Hydrocarbon Weathering...........................................13

Bacterial Biodegradation of Petroleum Hydrocarbons .....................14

Fungal Biodegradation of Petroleum Hydrocarbons...........................18

2.2.2 Polyaromatic Hydrocarbons (PAHs) ..................................................19

Toxicity of PAHs ..................................................................... 19

Abiotic Weathering Processes Affecting PAHs in Soil ......................20

Bacterial Biodegradation of PAHs ...............................................22

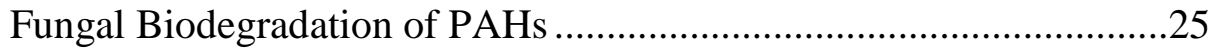

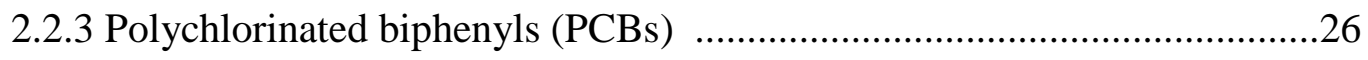

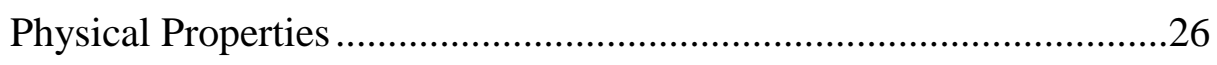

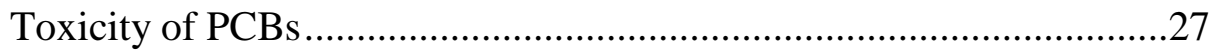


Abiotic Weathering of PCBs

Aerobic Pathways of Bacterial PCB Biodegradation .31

Fungal Biodegradation of PCBs

2.2.4 Dioxins (polychlorinated dibenzo-p-dioxins and dibenzofurans)

Physical Properties and Toxicity of Dioxins.

Abiotic Weathering Effects on Dioxins .36

Biodegradation of Dioxins .38

Bacterial Aerobic Mechanisms for Degradation of Lower

Chlorinated Dioxins .39

Fungal Biodegradation of Dioxins: White-rot fungi..... .40

2.3 TRFLP and qPCR Background 41

TRFLP Background

qPCR Background.

3.0 Natural Attenuation Rates and Microbial Degraders Reported in the Literature

3.1 Reported Rates of Natural Attenuation of Petroleum Hydrocarbons (non-

PAH)

3.2 Reported Rates of Natural Attenuation of PAHs

3.3 PCB Biodegradation 55

3.4 Dioxin Biodegradation .74

4.0 Methods .86 
4.1 Soil Sample Site Selection

4.2 DNA Extraction Protocol

4.3 TRFLP Analysis of Soil Samples

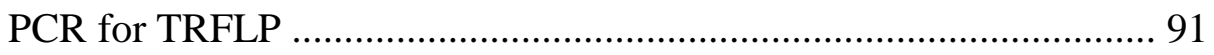

PCR Clean Up ..................................................................92

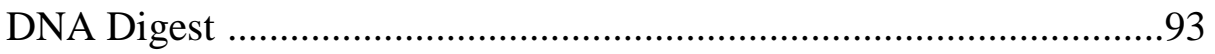

Ethanol Precipitation ..............................................................93

CEQ 8000 Sample Preparation ...................................................94

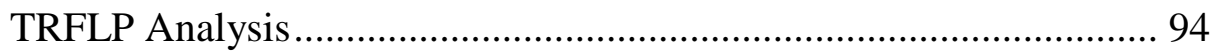

4.4 qPCR Analysis of Environmental Samples ............................................ 96

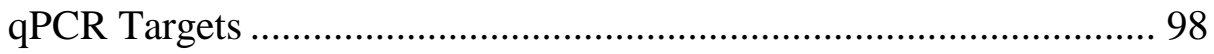

4.5 Culturing and 16S Sequencing of Microbes from Contaminated Site Soil

Media, Stock Solutions of Model Chemicals .................................................99

Inoculation Procedures: Plate Cultures ..........................................103

Inoculation Procedures: Liquid/Enrichment Cultures..................... 104

Culturing Positive Control Organisms ......................................... 106

DNA Extraction and Sequencing ................................................107

Sequencing and Data Analysis .................................................. 109

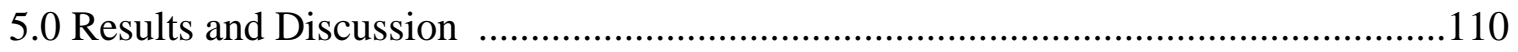

5.1 Soil Sample Locations and Contaminant Concentrations ........................ 110 


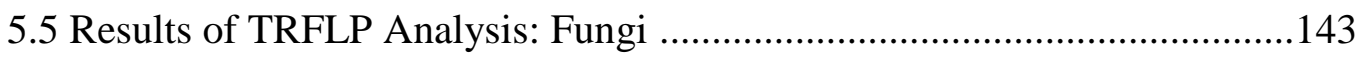

5.6 TRFLP MDS and Indices of Multivariate Dispersion Analysis.....

5.8 Cultured Potential Degraders and Control Organisms in TRFLP

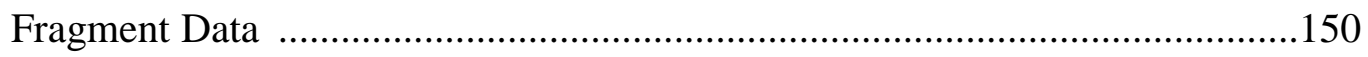

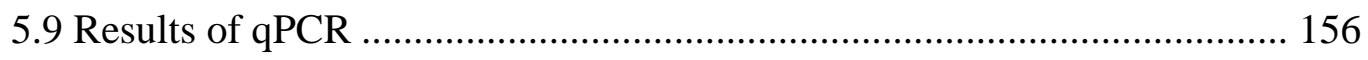

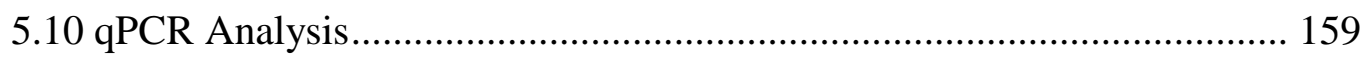

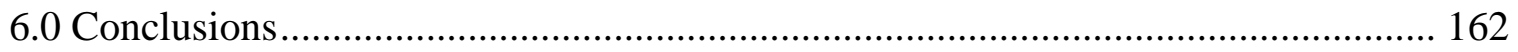

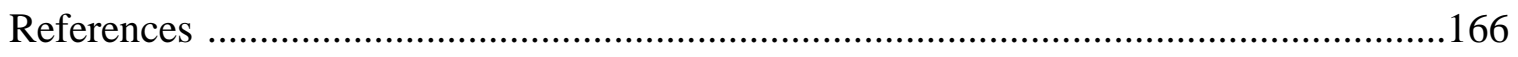

Appendices

Appendix A: Abbreviations 184

Appendix B: Additional MDS Scatter Plots .188 


\section{LIST OF TABLES}

Table 2.1: High and Low Concentrations of the Major Contaminants found in Area IV 8

Table 2.2: Soil Vapor Analysis 10

Table 2.3: A summary of microbial and fungal catabolism of polycyclic aromatic hydrocarbons (Gibson et al., 1990). Many of the end products shown here are easily biodegraded further to $\mathrm{CO} 2$ .15

Table 2.4: Dioxin toxic equivalency factors (TEFs) reported by the

World Health Organization (WHO) 36

Table 3.1: Biodegradation rates of petroleum hydrocarbons reported

in the literature (most cited field and laboratory studies)

Table 3.2: Reported Natural Attenuation Biodegradation Rates of PAHs in Soil .48

Table 3.3: Biodegradation Rates of PCBs under Natural Attenuation Conditions

Table 3.4: PCB-degrading bacterial species

Table 3.5: Summary of reported rates of dioxin biodegradation

under natural attenuation conditions. Initial and final concentrations

are listed in in $\mathrm{mg} / \mathrm{kg}$ or $\mathrm{ppm}$, and kinetics have been calculated

Table 3.6: Dioxin-degrading microorganisms cited in literature. Initial and

final concentrations were used to determine degradation rates .79

Table 4.1: Targets of QuantArray® Petro

Table 4.2: Composition and Recipes for Medias used in Culturing Experiments.......... 101

Table 4.3: Model Chemicals and Concentrations of Stock Solutions .102

Table 4.4: Positive Control Organisms for Culturing Experiments. 106 
Table 5.1: Chemical Data of the 30 Selected Sample Sites 112

Table 5.2: Bacterial Culturing Conditions and Results .114

Table 5.3: Fungal Culturing Conditions and Results .118

Table 5.4: Summary of Isolated Organisms 123

Table 5.5: Identified Organisms from Culturing Experiments .126

Table 5.6: Factors used in MDS Analysis of TRFLP Data .136

Table 5.7: Indices of Multivariate Dispersion for each Factor: Bacteria .139

Table 5.8: Indices of Multivariate Dispersion for each Factor: Fungi. 144

Table 5.9: Cultured Organisms in TRFLP Fragment Analysis. 152

Table 5.10: Summary of the QuantArray® Petro qPCR results .158 


\section{LIST OF FIGURES}

Figure 2.1: Contour Map of Area IV Subareas 5B and 5C showing the concentrations of the major contaminants .11

Figure 2.2: General pathway of aerobic degradation of small alkanes and other hydrocarbons .18

Figure 2.3: A summary of microbial and fungal catabolism of polycyclic aromatic hydrocarbons (Gibson et al., 1990). Many of the end products shown here are easily biodegraded further to $\mathrm{CO} 2$

Figure 2.4: Generic Structure of PCBs (ASTDR, 2010). Positions 2,2',6, and 6' are ortho positions, positions 3,3',5 and 5' are meta positions and positions 4 and 4' are para positions

Figure 2.5: Chemical structures of Dibenzo-p-dioxins (DD) and

dibenzofurans (DF), showing analogues and numbering conventions

Figure 5.1: Soil Sample Sites on SSFL

Figure 5.2: Some Plates with Colonies. The left image is a

Bushnell Haas plate inoculated with soil from site D02 and spiked with 1 ppm of DBZ showing fungal and bacterial colonies. The right image is a Bushnell Haas plate inoculated with soil from site T02 and spiked with $500 \mathrm{ppm}$ of naphthalene showing fungal colonies .125

Figure 5.3: An example of a TRFLP chromatogram

Figure 5.4: MDS from bacterial fragments using COI series as a factor.

T is for the TPH/PAH sample set, $\mathrm{P}$ is for the PCB sample set, and D is for the dioxin sample set 
Figure 5.5: MDS from bacterial fragments with TPH bubbles based on TPH concentrations

Figure 5.6: MDS from bacterial fragments with PAH bubbles based on concentrations of PAHs

Figure 5.7: MDS from bacterial fragments with PCBs bubbles based on $\log$ of PCB concentrations 142

Figure 5.8: MDS from bacterial fragments with dioxin bubbles based on dioxin concentrations

Figure 5.9: MDS from fungal fragments with TPH bubbles based on TPH concentrations

Figure 5.10: MDS from fungal fragments with PAH bubbles based on concentrations of PAHs 146

Figure 5.11: MDS from fungal fragments with PCBs bubbles based on $\log$ of PCB concentrations 146

Figure 5.12: MDS from fungal fragments with dioxin bubbles based on dioxin concentrations 


\subsection{Introduction}

Natural attenuation is one of several strategies being considered for the remediation of contaminated soil. The US EPA defines natural attenuation as the "use of natural processes to contain the spread of the contamination from chemical spills and reduce the concentration and amount of pollutants at contaminated sites (US EPA 2013).” These natural processes include biological degradation by existing bacteria, plants and fungi, as well as abiotic processes such as volatilization, dispersion, dilution, and sorption of contaminants onto organic matter and clay minerals in the soil. It is a viable and cost effective way to remediate contaminated soil on some sites under certain circumstances (Wiedemeier et al. 1999). The use of natural attenuation requires providing certain "lines of evidence" that processes such as biodegradation are contributing to reductions of contaminated concentrations. Also, the monitoring of a site during natural attenuation is essential to ensure there is no risk to the environment or public health. This study examines the applicability of natural attenuation to the Santa Susana Field Laboratory site in Southern California where rocket testing and energy development research led to soil contamination. The goal of the study is to determine if the constituents in SSFL soil be reduced to acceptable levels using natural attenuation. Contaminants of interest (COI) at this site include petroleum hydrocarbons (PHCs), polycyclic aromatic hydrocarbons (PAHs), dioxins, polychlorinated biphenyls (PCBs), perchlorate and various metals (mercury in particular). These contaminants are known to have adverse effects on human health (ASTDR; U.S. Department of Health and Human Services 1999; ATSDR 2009; Kang et al. 1991). DNA analyses, including TRFLP and qPCR, culturing and sequencing experiments, and microcosm experiments (part of a companion study) were chosen as the 
best options for determining if natural attenuation of COIs at the site is plausible. TRFLP was used to compare microbial communities, qPCR was used to quantitate specific genes, and culturing was used to determine if native soil microbes could degrade the COIs.

TRFLP analysis was selected because of its ability to compare microbial populations for both bacteria and fungi and relate them to various characteristics of the samples (Kaplan and Kitts 2004). This analysis was used to characterize the microbial communities in the soil and relate differences between these communities to soil type, COI series, sample location, and the presence/absence of each COI. Determining if the presence or concentration of COIs has an effect on microbial population is important in predicting if natural attenuation is possible for two reasons. If the presence of a COI dramatically changes the microbial population then there is likely to be either biodegradation occurring or toxicity effects. Both of these are important aspects of the soil environment. Comparison of observed TRFLP patterns to libraries of TRFLP patterns for known microorganisms were also used to infer the presence of certain types of microorganisms.

qPCR was selected because of its ability to quantify specific organisms and genes known to be involved in biodegradation (Udvardi, Czechowski, and Scheible 2008). Copies of a gene associated with biodegradation of a COI not only have to exist in the microbial population, but they need to exist in large enough quantities to significantly change the concentration of a COI; qPCR provides this information. Additionally, many targets can be selected for specific microbial metabolic activities that can reveal which COIs can be biodegraded and which ones can't. Per the recommendations of Microbial Insights ${ }^{\circledR}$, the analyses selected were the QuantArray® Petro and the Census: Dehalococcoides. The 
QuantArray ${ }^{\circledR}$ Petro included 18 targets for PHCs and PAH degradation, including biphenyl dioxygenase which is involved in PCB biodegradation.

Finally, culturing was selected because it is a classic method that can be used to show that microbes obtained directly from the site can grow on the contaminants. This is important, since many molecular methods rely on the DNA only, and sometimes only show that the genes necessary are present but not if they are being used. By sequencing the isolates it can be further confirmed if the microbes on the site are shown by other researchers to degrade the COIs. Spiked Bushnell Haas media was used to isolate potential COI degraders from soil from the site. For each COI a model compound was chosen and used to select for the growth of degraders of that COI. Isolated colonies were grown up in liquid media and sequenced. Bacteria colonies were identified using $16 \mathrm{~S}$ sequencing, while fungi were identified using sequences of the ITS region. These identified microbes were compared to the literature to determine if they are known degraders of that $\mathrm{COI}$.

Metagenomics was also explored for this application, but unfortunately Sandia Labs was unable to run the samples before this thesis was completed. It was determined that the results of these experiments would provide enough lines of evidence to determine if natural attenuation is occurring on the site and give an estimate for timeframe for remediation. Metagenomics would provide further detail to these results. These analyses were to be carried out on 30 soil samples from the site that were selected based on the site characterization data. 
Two companion studies are being conducted at Cal Poly San Luis Obispo by graduate students Mackenzie Billings and Matt Poltrak. These studies include microcosm experiments which will be used to study the feasibility of bioremediation and phytoremediation techniques on the site and determine rates of COI degradation occurring in soil from SSFL in a lab environment. The findings of this study will be used to inform the other studies and guide decisions within them. 


\subsection{Background}

\subsection{SSFL Site Background and Project Origin}

The SSFL site has a long history of activities and events that led to soil contamination with various chemicals ("Boeing: Santa Susana” 2014a). North American Aviation (NAA) established SSFL in 1947 for static-fire tests on large rocket engines ("Boeing: Santa Susana” 2014b). The site was used for government and commercial research and development of both nuclear technology liquid-fuel rocket engines. These two divisions eventually separated in 1955 into Atomics International (AI) and Rocketdyne, respectively. Rocket testing was conducted on the site continuously from 1950 to roughly 2000. These tests were performed by NASA, USAF, Rocketdyne, NAA, and Boeing. Area IV is a 290 -acre section of SSFL that was used for nuclear and energy research and development, led by AI and the US Department of Energy (DOE). In 1954 AI (as a subsidiary of NAA) began using Area IV of SSFL for energy research. Ninety of the 290 acres in Area IV were leased to the Atomic Energy Commission (AEC) and subsequently to DOE. This 90-acre portion was named the Energy Technology Engineering Center (ETEC), and was used for nuclear energy research as well as other research projects. The ETEC was also the site of DOE's Liquid Metals Center of Excellence. During this time commercial clients, supported by AI, conducted research on nuclear energy (Chew 2006). ETEC's operations included 10 small nuclear reactors and the operation of one of the first commercially available hot laboratories, which was used for the assessment and processing of nuclear fuels (SSFL WG 2014; Chew 2006). Sodium or other liquid metals (including mercury) were tested as coolants in these reactors as alternatives to water or gas. One of the test reactors overheated and the power plant experienced partial core 
melts releasing their coolant and other materials including radionuclides (California Energy Comission 2012). The last non-nuclear research in Area IV was halted in 2001 with the closure of the Sodium Pump Test Facility. Since then all nuclear materials have been removed from ETEC and only a few shells of buildings remain. The Radioactive Materials Handling Facility (RMHF) is the only remaining active facility and will assist in the final building demolition and soil cleanup operations in the event that radioactive materials are found during the cleanup process of SSFL (Chew 2006).

In 2005 the Topanga Wildfire burned almost all of the brush on the SSFL site, and the surrounding Simi Hills. The fire burned 24,000 acres, including 2,000 of the 2850 acres of SSFL (roughly $80 \%$ of the site) (ETEC 2005; Chew 2006). Some buildings sustained substantial fire damage, about 10 out of the 200 on the site. During the fire, roughly 150 pounds of Freon ${ }^{\circledR}$ were lost from air-conditioning units (ETEC, 2005). The effects of the fire on the contaminants are largely unknown. However, fires are known to produce dioxins, particularly when the fuel source has high chlorine concentrations (Thomas and Sprio 1994). Due to the large release of Freon (a chlorinated compound) during the fire, it is possible that more dioxins were produced (ETEC 2005).

During the various research projects, activities, and events that occurred in Area IV and in other areas of SSFL, a large number of chemicals were used. PCBs were used in electrical components such as transformers. Various hydraulic fluids and fuels were used to run generators, heat water for steam, and other applications. Solvents were used to clean parts during and after tests. Mercury and sodium were used in energy transfer applications, and silver was used in photograph development. Waste was burned on the site, which produced dioxins and released the PCBs, metals, fuels and lubricants, and 
solvents from transformers, storage tanks, drums in storage areas and at leach fields. Soil sampling and chemical analysis has determined the areas and extent of the contamination. The ranges of the contaminants found in the soil of Area IV are shown in Table 2.1. The locations with the highest contamination are shown in Figure 2.2. Soil vapor analysis was also performed at 18 sites to determine if the soil was aerobic or anaerobic. Gas was extracted from between 5 and 20.5 feet depending on the sample site. It was concluded that the soil is completely aerobic (Table 2.2).

In May 2011 the DOE contracted Sandia National Laboratories (Sandia) to initiate the treatability study process. Sandia evaluated the options for soil treatability and made recommendations for the best technology options applicable to Area IV. Sandia recommended natural attenuation, bioremediation, phytoremediation, and soil partitioning be considered as potential technologies to use at Area IV. To explore the feasibility of these approaches, the DOE has commissioned five treatability studies to address the soil contamination and determine the best way to restore the site to reasonable levels for health and safety. California Polytechnic State University was awarded the Natural Attenuation, Bioremediation, and Phytoremediation Studies. The methods, findings, and conclusions of the Natural Attenuation Study and part of the companion study on Bioremediation performed by California Polytechnic State University will be discussed here.

The Natural Attenuation Study consisted of an extensive literature review that resulted in a list of bacteria and fungi that can degrade petroleum hydrocarbons, PAHs, PCBs, and dioxins as well as the chemical mechanisms they use. It also produced a list of rates for all four chemical classes from both field and laboratory experiments. A collection of in- 
situ field studies and ex-situ lab studies provided natural attenuation rates observed in the field. A comparison of historical and current chemical data from the site was also performed to provide a line of evidence for natural attenuation.

Table 2.1. High and Low Concentrations of the Major Contaminants found in Area IV (CDM Smith, 2012).

\begin{tabular}{|c|c|c|c|c|}
\hline Contaminant & Class & $\begin{array}{c}\text { Lowest } \\
\text { Measured } \\
\text { Concentration } \\
\end{array}$ & $\begin{array}{l}\text { Highest Measured } \\
\text { Concentration }\end{array}$ & Units \\
\hline 2,3,7,8-TCDD TEQ & Dioxin & 52 & 650 & ppt \\
\hline TCDD TEQ & Dioxin & 9.1 & 292.4 & ppt \\
\hline Aluminum & Metal & 30500 & 31900 & ppm \\
\hline Antimony & Metal & 11.8 & 870 & ppm \\
\hline Arsenic & Metal & 24.6 & 350 & ppm \\
\hline Barium & Metal & 158 & 1000 & ppm \\
\hline Beryllium & Metal & 1.17 & 1.3 & ppm \\
\hline Boron & Metal & 17.9 & only 1 measurement & $\mathrm{ppm}$ \\
\hline Cadmium & Metal & 1.6 & 18.6 & ppm \\
\hline Chromium & Metal & 38.3 & 693 & ppm \\
\hline Chromium (hex) & Metal & 0.3 & 4.8 & ppm \\
\hline Cobalt & Metal & 27 & 48 & $\mathrm{ppm}$ \\
\hline Copper & Metal & 60 & 699 & ppm \\
\hline Lead & Metal & 41 & 27000 & ppm \\
\hline Lithium & Metal & 53.9 & only 1 measurement & ppm \\
\hline Manganese & Metal & 1010 & only 1 measurement & ppm \\
\hline Mercury & Metal & 0.127 & 53.8 & ppm \\
\hline Nickel & Metal & 37.5 & 538 & ppm \\
\hline Selenium & Metal & 0.727 & 2.43 & $\mathrm{ppm}$ \\
\hline Silver & Metal & 2.18 & 420 & ppm \\
\hline Thorium & Metal & 0.484 & 2.4 & ppm \\
\hline Vanadium & Metal & 63 & 93.3 & ppm \\
\hline Zinc & Metal & 160 & 3400 & $\mathrm{ppm}$ \\
\hline 1-Methylnaphthalene & PAH & 7000 & only 1 measurement & $\mathrm{ppb}$ \\
\hline 2-Methylnaphthalene & PAH & 12000 & only 1 measurement & $\mathrm{ppb}$ \\
\hline
\end{tabular}




\begin{tabular}{|c|c|c|c|c|}
\hline Contaminant & Class & $\begin{array}{c}\text { Lowest } \\
\text { Measured } \\
\text { Concentration } \\
\end{array}$ & $\begin{array}{l}\text { Highest Measured } \\
\text { Concentration }\end{array}$ & Units \\
\hline Benzo(a) anthracene & PAH & 20.8 & 16000 & $\mathrm{ppb}$ \\
\hline Benzo(a)pyrene & PAH & 29 & 31000 & $\mathrm{ppb}$ \\
\hline Benzo(b) fluoranthene & PAH & 28.3 & 26000 & $\mathrm{ppb}$ \\
\hline $\operatorname{Benzo}(\mathrm{g}, \mathrm{h}, \mathrm{i})$ perylene & $\mathrm{PAH}$ & 42.6 & 90000 & $\mathrm{ppb}$ \\
\hline Benzo(k) fluoranthene & $\mathrm{PAH}$ & 0.056 & 15000 & $\mathrm{ppb}$ \\
\hline Chrysene & $\mathrm{PAH}$ & 23.8 & 22000 & $\mathrm{ppb}$ \\
\hline $\begin{array}{c}\text { Dibenzo(a,h) } \\
\text { anthracene }\end{array}$ & PAH & 27 & 3900 & $\mathrm{ppb}$ \\
\hline Fluoranthene & $\mathrm{PAH}$ & 44 & 32000 & $\mathrm{ppb}$ \\
\hline Ideno(1,2,3-cd) pyrene & $\mathrm{PAH}$ & 46.3 & 77000 & $\mathrm{ppb}$ \\
\hline Naphthalene & $\mathrm{PAH}$ & 170 & only 1 measurement & $\mathrm{ppb}$ \\
\hline Phenanthrene & PAH & 65 & 14000 & $\mathrm{ppb}$ \\
\hline Pyrene & PAH & 114 & 27000 & $\mathrm{ppb}$ \\
\hline Aroclor 1248 & PCB & 34 & 24000 & $\mathrm{ppb}$ \\
\hline Aroclor 1254 & PCB & 19 & 8090 & $\mathrm{ppb}$ \\
\hline Aroclor 1260 & PCB & 15.7 & 630000 & $\mathrm{ppb}$ \\
\hline TPH- Lubricant Oil & $\mathrm{TPH}$ & 170 & 82000 & ppm \\
\hline TPH-C12-C14 & $\mathrm{TPH}$ & 36 & only 1 measurement & $\mathrm{ppm}$ \\
\hline TPH-C15-C20 & $\mathrm{TPH}$ & 44 & only 1 measurement & ppm \\
\hline TPH-C30-C40 & $\mathrm{TPH}$ & 130 & 5100 & $\mathrm{ppm}$ \\
\hline TPH-Diesel & $\mathrm{TPH}$ & 118 & 8300 & ppm \\
\hline TPH-Gasoline Range & $\mathrm{TPH}$ & 3 & 6.6 & ppm \\
\hline TPH-Kerosene Range & TPH & 138 & 350 & ppm \\
\hline
\end{tabular}


Table 2.2: Soil Vapor Analysis

\begin{tabular}{|c|c|c|c|c|}
\hline \multirow{2}{*}{$\begin{array}{c}\text { Depth } \\
\text { (ft } \\
\text { bgs) }\end{array}$} & \multicolumn{2}{|c|}{$\mathrm{CO}_{2}$} & \multicolumn{2}{|c|}{$\mathrm{O}_{2}$} \\
\hline & average & st. dev. (\% of avg) & average & st. dev. (\% of avg) \\
\hline 5 & 0.015 & $74 \%$ & 0.191 & $5 \%$ \\
\hline 6 & 0.007 & $60 \%$ & 0.195 & $5 \%$ \\
\hline 7 & 0.027 & $62 \%$ & 0.18 & $7 \%$ \\
\hline 8 & 0.013 & $53 \%$ & 0.185 & $7 \%$ \\
\hline 9 & 0.058 & $\mathrm{n} / \mathrm{a}$ & 0.127 & $\mathrm{n} / \mathrm{a}$ \\
\hline 10 & 0.02 & $117 \%$ & 0.178 & $10 \%$ \\
\hline 11 & 0.049 & $\mathrm{n} / \mathrm{a}$ & 0.164 & $\mathrm{n} / \mathrm{a}$ \\
\hline 12 & 0.02 & $\mathrm{n} / \mathrm{a}$ & 0.188 & $\mathrm{n} / \mathrm{a}$ \\
\hline 13 & no measurement & no measurement & no measurement & no measurement \\
\hline 14 & 0.01 & $\mathrm{n} / \mathrm{a}$ & 0.159 & $\mathrm{n} / \mathrm{a}$ \\
\hline 15 & 0.059 & $\mathrm{n} / \mathrm{a}$ & 0.144 & $\mathrm{n} / \mathrm{a}$ \\
\hline 16 & 0.016 & $\mathrm{n} / \mathrm{a}$ & 0.19 & $\mathrm{n} / \mathrm{a}$ \\
\hline 17 & no measurement & no measurement & no measurement & no measurement \\
\hline 18 & no measurement & no measurement & no measurement & no measurement \\
\hline 19 & 0.046 & $\mathrm{n} / \mathrm{a}$ & 0.162 & $\mathrm{n} / \mathrm{a}$ \\
\hline 20 & 0.061 & $\mathrm{n} / \mathrm{a}$ & 0.141 & $\mathrm{n} / \mathrm{a}$ \\
\hline
\end{tabular}




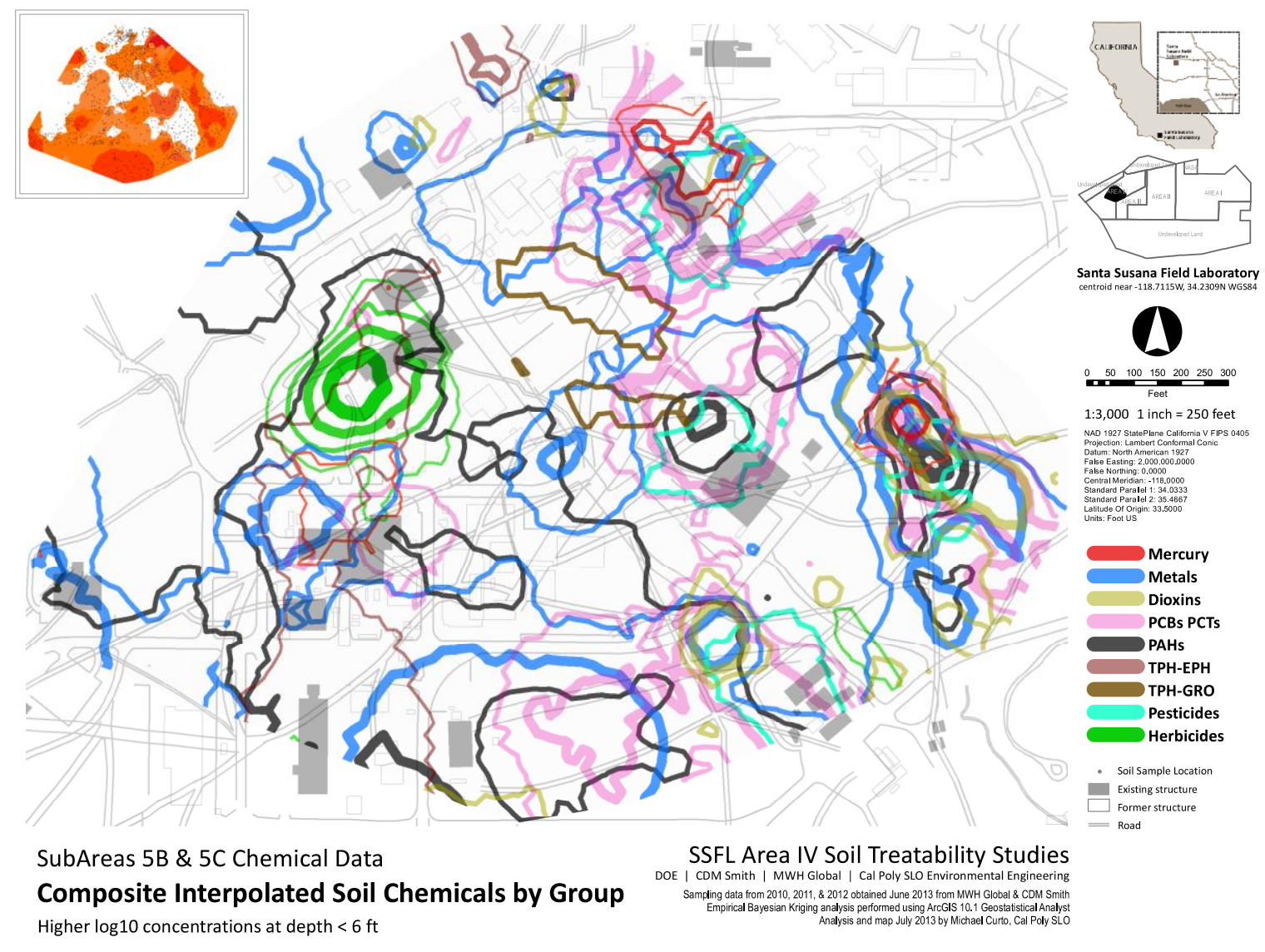

Figure 2.1. Contour Map of Area IV Subareas 5B and 5C showing the concentrations of the major contaminants (CDM Smith 2013b)

\subsection{Contaminants of Interest}

The four main classes of chemicals have unique properties, which creates the variability in their toxicities, degradation pathways, and weathering. This also means that different bacteria and/or fungi are needed to degrade different chemicals. It is important to look at each chemical class in order to understand which microbes can degrade them and how. 


\subsubsection{Petroleum Hydrocarbons (PHCs)}

\section{Physical Properties}

Petroleum hydrocarbons include mixtures of hydrocarbons found in crude oil and refined fuels. Technically this includes polycyclic aromatic hydrocarbons (PAHs), but these are considered separately in the next section. There are four main classes of the over 17,000 organic compounds found in oil: saturated hydrocarbons, aromatic hydrocarbons, asphaltenes (phenols, fatty acids, ketones, esters, and porphyrins), and resins (pyridines, quinolines, carbazoles, sulfoxides, and amides) (Marshall \& Rodgers, 2008). The contaminants of interest at SSFL include both saturated and aromatic hydrocarbons. Aromatics will primarily be addressed in the discussion of PAHs. Because the contaminants at the site are extensively weathered, benzene, toluene, ethylbenzene, and xylene (BTEX) are likely to have evaporated and/or biodegraded and thus are not discussed in this report.

Total petroleum hydrocarbon (TPH) is a term used for the collective quantification of petroleum hydrocarbons. TPH can be determined in ranges of equivalent carbon atoms per molecule. For example, TPH C12-C14 is a measurement of hydrocarbons with the equivalent of 12 to 14 carbons in terms of when they elute in a gas chromatogram. Hydrocarbons are generally hydrophobic. The longer the carbon chains, the more hydrophobic the compound. Most of the constituents in petroleum hydrocarbon mixtures have relatively high vapor pressures and low solubilities in water (PRO-ACT 1999).

Soil contamination by petroleum hydrocarbons is common due to human activity and accidents such as fuel and oil spills (Brooijmans, Pastink, \& Siezen, 2009). TPH has been 
measured in Area IV of SSFL at concentrations up to 82,000 ppm, but most of the soils in the clearly contaminated areas have TPH concentrations between 100 and 1,000 ppm.

Petroleum compounds have a range of toxic effects, including developmental, hematological, hepatic, immunological, and renal disturbances (“ATSDR - Toxic Substances - Total Petroleum Hydrocarbons (TPH),"). Concern about PHC exposure is primarily related to BTEX and PAHs. PAHs will be discussed later. There are known effects and established minimal risk levels for acute, intermediate, and chronic exposure to hydrocarbons. Toxicity from hydrocarbon ingestion most often affects the lungs (Levine, 2013). Neurological, respiratory, reproductive, and renal effects are associated with exposure to aliphatic hydrocarbons with 5-8 carbons; those with 8-35 carbons are associated with hepatic, adaptive, and metabolic effects (U.S. Department of Health and Human Services, 1999).

\section{Petroleum Hydrocarbon Weathering}

Petroleum compounds typically "weather" in the environment, meaning some components of the petroleum hydrocarbon mixture are either removed or transformed over time. Weathering processes include abiotic processes such as volatilization, chemical or photochemical oxidation, and adsorption into the pore structure of the soil, and biological processes such as biodegradation as described below. Volatilization may decrease the amounts of smaller hydrocarbons that have a higher vapor pressure. This primarily affects gasoline- and kerosene-range hydrocarbons and hydrocarbons in the C10-C16 range (Nishiwaki et al., 2011). Previous studies on diesel-contaminated soil show that volatilization can account for up to $2 \%$ of initial TPH concentration reduction 
(Namkoong et al., 2002a). Also, preferential biodegradation of the most biodegradable hydrocarbon substrates results in a change in composition, with weathered petroleum spills typically depleted of straight-chain alkanes (Whittaker \& Pollard, 1997).

Weathering processes can hinder bioremediation through sequestration of contaminants in the soil. Hydrocarbons are slowly absorbed into the organic phase of the soil, which can significantly reduce their bioavailability resulting in lower biodegradation rates (Gallego et al., 2010).

\section{Bacterial Biodegradation of Petroleum Hydrocarbons}

Petroleum hydrocarbon biodegradation by naturally occurring microflora is very well documented (Atlas, 1981; F. Bento et al., 2005; Sarkar et al., 2005; and Gieg et al., 1999). Aerobic bacteria are reported to perform the vast majority of biodegradation, but yeast and fungi also biodegrade hydrocarbons (Rahman et al., 2003 and Brooijmans et al., 2009). Common genera of hydrocarbon-degrading bacteria include Pseudomonas, Acinetobacter, Burkholderia, Mycobacterium, Haemophilus, Rhodocoicus, Paenibacillus, and Ralstonia and numerous other genera (Tyagi, da Fonseca, and de Carvalho 2011a; Margesin et al., 2003; and Das \& Chandran, 2011).

Petroleum hydrocarbons have a wide range of chemical properties and thus exhibit a wide range of biodegradation rates. The New Zealand Ministry for the Environment has cited benchmark half lives for different classes of hydrocarbons and several PAHs (Table 2.3). These rates can serve as a general guide for biodegradability of the different fractions. More specific published rates are presented below in Section 3.5. Clearly, short-chain and aliphatic hydrocarbons biodegrade faster than aromatic compounds. 
Linear and branched alkanes are particularly susceptible to microbial biodegradation (Ministry for the Environment 1996). This means that weathered petroleum contamination usually consists of longer chain and aromatic compounds, which are more difficult to biodegrade.

Table 2.3. Benchmark half lives adopted by the New Zealand Ministry for the Environment (Ministry for the Environment 1996)

\begin{tabular}{|c|c|}
\hline Hydrocarbon Fraction & Half Life \\
\hline C7-C9 & 2 years \\
\hline C10-C14 & 5 years \\
\hline C15-C36 & 10 years \\
\hline Naphthalene & 5 years \\
\hline Pyrene & 10 years \\
\hline Benzo(a)pyrene & 10 years \\
\hline
\end{tabular}

The mechanism of aerobic hydrocarbon biodegradation is oxidation mediated by enzymes such as monooxygenase, dioxygenase and peroxidase as well as cytochrome p450 systems (van Beilen et al. 2003). In aerobic biodegradation of petroleum hydrocarbons, monooxygenase enzymes typically attack alkanes, while dioxygenase enzymes attack aromatic compounds, both mechanisms using oxygen as an oxidizing 
agent (Das \& Chandran, 2011 and Wiedemeier et al., 1999). Oxidation of alkanes and many other compounds produces alcohols, aldehydes, epoxides and carboxylic acids. These compounds are then completely broken down and the carbon is either respired as carbon dioxide or incorporated into cell biomass (Figure 2.3).

There are several organisms that express enzymes related to monooxygenases that have a very narrow substrate range (for example, methane monooxygenase metabolizes methane). For the most part, these enzymes are responsible for oxidizing $\mathrm{C} 1$ through $\mathrm{C} 4$ hydrocarbons (van Beilen and Funhoff 2005). Microbes with these enzymes are fairly specialized and will not likely play a large role in the natural attenuation of the longer hydrocarbons at SSFL. A mechanism more likely to occur in SSFL soils is carried out by particulate alkane hydrolayses like those expressed in $P$. putida GPo1, which preferentially oxidize alkanes longer than $\mathrm{C} 10$.

Two classes of alkane-hydroxylating p450 systems have also been identified. Class 1 p450s consist of a three-component system comprised of cytochrome $\mathrm{p} 450$, ferredoxin, and ferredoxin reductase subunits (van Beilen and Funhoff 2007). Class 2 p450s have a microsomal 2-component system comprised of a membrane-bound p450 and a reductase. These are found in various soil yeast strains and oxidize n-alkanes to yield fatty acids and carboxylic acids. The most active of the p450 enzymes is p450BM-3. The Alk B gene, which is required for p450 enzymatic activity, is present in M. tuberculosis, Prauserella rugosa, Rhodocoicus erythropolis, Burkholderia cepacia, Pseudomonas aeruginosa, Acinetobacter sp. and Alcanivorax borkumensis, organisms that are prevalent in soil (van Beilen and Funhoff 2005). They have a wide range of substrates from C5 - C12, and others can oxidize C10 - C16 alkanes. 
Anaerobic petroleum hydrocarbon biodegradation has been studied far less than aerobic biodegradation (Wiedemeier et al., 1999). In order for anaerobic degradation to occur, both alternative electron acceptors and microorganisms that are able to use them must be present (Ulrich \& Suflita, 2001). Hydrocarbon constituents have been shown to biodegrade under Fe(III)-reducing, denitrifying, and sulfate-reducing conditions, and manganese oxides, soil humic acids, and fumarate have also been implicated in anaerobic hydrocarbon biodegradation (Van Hamme et al., 2003 and Townsend et al., 2003). Both facultative anaerobes (nitrate-, iron-, and manganese-reducing microorganisms) and strict anaerobes (e.g. sulfate-reducers) can biodegrade hydrocarbons anaerobically (Grishchenkov et al., 2000). However, compared to aerobic biodegradation, anaerobic biodegradation lends itself to fewer hydrocarbon substrates at much lower rates and to a lesser extent (Grishchenkov et al., 2000). A study by one of the participators of this research (Nelson) concluded that anaerobic degradation of petroleum compounds in groundwater at the former Guadalupe Oil Field was extremely slow compared to aerobic biodegradation (Chell et al., 2007). 


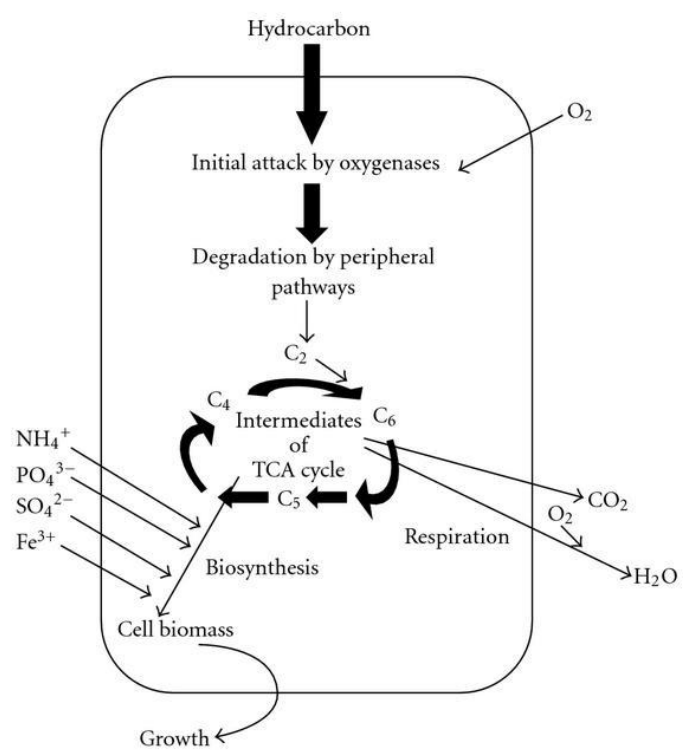

Figure 2.2: General pathway of aerobic degradation of small alkanes and other hydrocarbons (Das \& Chandran, 2011).

\section{Fungal Biodegradation of Petroleum Hydrocarbons}

Fungi are also common degraders of hydrocarbons. White-rot fungi (Phanerochaete sp.) have been shown to effectively biodegrade a wide variety of hydrocarbon compounds (Pointing, 2001). Ligninolytic enzymes are thought to be primary contributors in fungal breakdown of petroleum hydrocarbons. Most of the research on fungi such as white-rot fungi has been done for more recalcitrant compounds than alkanes, such as PAHs and PCBs (Pointing, 2001).

In a study conducted by Yateem et al. (1998), the fungi Phanerochaete chrysosporium, Pleurotus ostreatus, and Coriolus versicolor were tested for their ability to degrade petroleum hydrocarbons in soil microcosms. The results indicated that Coriolus versicolor was the most active degrader. After 12 months, $78.1 \%$ of TPH was 
biodegraded under nitrogen-rich conditions. P. chrysosporium removed $77.1 \%$ under nitrogen-limiting conditions.

\subsubsection{Polyaromatic Hydrocarbons (PAHs)}

\section{Physical Properties}

Polyaromatic hydrocarbons, also known as polynuclear aromatic hydrocarbons, are hydrocarbons with multiple aromatic rings (usually between 2 and 10), which do not contain heteroatoms or substituents. They have low solubility in water, which decreases as molecular weight increases (“ChemSpider" 2013; N. K. Nagpal 1993). They typically have high melting and boiling points and low vapor pressures. Melting and boiling points increase at higher molecular weights, while vapor pressure decreases (Haritash \& Kaushik, 2009). Information about molecular weight, formula, structure, solubility, vapor pressure, Log Kow, carcinogenicity, and number of rings of each PAH found in Area IV is reported in "ChemSpider," 2013 and Nagpal, 1993. PAHs are common airborne pollutants produced from burning fuel. They also occur naturally in oil, coal, and tar deposits. Their close link to fossil fuel processing and combustion makes them one of the most common organic pollutants (Lindsey et al., 1989). Many PAHs are known carcinogens, teratogens, and/or mutagens, and are therefore important to monitor (Srivastava et al., 2010). Since they are largely insoluble in water, air pollution is the primary concern for this group of hydrocarbons.

\section{Toxicity of PAHs}

Thirteen of the 15 PAHs found in Area IV are on the EPA's list of 127 Priority Pollutants (EPA, 2013). The toxicity of different PAHs is largely dependent on their chemical 
structure. The main concern of PAHs is their carcinogenic properties. The carcinogenic properties of PAHs are believed to be due to the binding of reactive PAH metabolites directly to DNA (ATSDR, 2009). These compounds are known mutagens that disrupt cell replication and bind to DNA, forming adducts (ATSDR, 2009).

\section{Degradation of PAHs}

Six possible fates of PAHs in the environment were reported by Wild \& Jones (1995): volatilization, adsorption on soil particles, photo-oxidation, chemical oxidation, leaching, or microbial degradation (bacterial and fungal). These abiotic and biotic processes are discussed in the sections below, and an assessment is made of their potential effect on the natural attenuation of PAHs at the SSFL site.

\section{Abiotic Weathering Processes Affecting PAHs in Soil}

Volatilization: Volatilization of PAHs from soil is likely only for PAHs with higher vapor pressures, such as naphthalene and methyl-naphthalenes. Experiments by Park et al. (1990) showed that naphthalene and 1-methyl naphthalene accounted for 30 and 20\% of the reductions, respectively. Volatilization of all other PAHs in the study was negligible because of their low vapor pressure (Park et al., 1990). The wildfire at SSFL in 2005 may have aided in the volatilization of some PAHs in the top few inches of the soil.

Adsorption to soil matrix: Weathering of PAHs for long periods of time (5-10 years or more) causes PAHs to become absorbed into the organic phase of soil (Pierzynski, Vance, and Sims 2005). This reduces the total toxicity of the contaminants in the soil, which relieves stress on the microbial community. However, at the same time it greatly 
reduces the bioavailability of these compounds, reducing the potential for their bioremediation (Alexander, 1995).

Photo-oxidation: Photo-oxidation of PAHs can be significant in aquatic environments, but is not thought to be significant in terrestrial environments (Vilanova et al., 2001). Photo-oxidation requires direct exposure to sunlight, which is likely only in the top few $\mathrm{mm}$ of soil, and thus it is unlikely to be a significant mechanism for SSFL soils.

Chemical oxidation: Abiotic chemical oxidation can degrade PAHs in significant amounts depending on the size of the PAH. For PAHs with 3 or fewer rings chemical oxidation can account for $2-20 \%$ of the total reduction. However, for PAHs that have more than 3 rings, chemical oxidation is not a significant reduction mechanism (Park et al., 1990). Ozone and hydrogen peroxide are the most commonly used chemical oxidants for remediation, and must be added to the soil. These additions can be performed in situ or ex situ (Lundstedt, 2003).

Leaching: Leaching of PAHs from soil into groundwater or surface water is limited by the low solubility of PAHs, particularly for higher molecular weight PAHs. In one study naphthalene and phenanthrene were reported to dissolve into water and be leached out of soil, while larger, more hydrophobic PAHs became bound to colloids, which could also be leached through the soil (Bergendahl, 2005). The study showed $80-90 \%$ of the PAHs in the leachate had 3 rings or less, while only constituting $18-25 \%$ of the PAH content in the soil. This further shows that smaller PAHs are preferentially leached from soils.

Biological weathering: Biodegradation can reduce the concentrations of PAHs in soil over time (see Sections 4.3 and 4.4 below). During this biological weathering most of the 
lower molecular weight PAHs are biodegraded early, leaving higher molecular weight fractions in the soil. This process has undoubtedly occurred at the SSFL, and needs to be accounted for in the plan for remediation of the site.

\section{Bacterial Biodegradation of PAHS}

PAHs biodegrade slowly under natural conditions, with the larger the PAHs (large number of rings) degrading particularly slowly (Haritash \& Kaushik, 2009). Along with the chemical structure of the $\mathrm{PAH}$, environmental conditions such as $\mathrm{pH}$, temperature, and oxygen availability have a large impact on biodegradation rates. These conditions are often interrelated and their effects are difficult to predict (Haritash \& Kaushik, 2009). In biological degradation, the bacterial and/or fungal species present and their population size is key (Haritash and Kaushik 2009). Other factors include microbial acclimation, nutrient accessibility, cellular transport, and chemical partitioning (Haritash \& Kaushik, 2009). Numerous PAH-degrading bacterial and fungal species have been isolated from PAH-contaminated soil (Jacques et al., 2009). Mechanisms of bacterial PAH biodegradation are described in this section, while fungal biodegradation is described in the following section. The New Zealand Ministry for the Environment has cited benchmark half lives for different PAHs (Table 2.3), and these rates can serve as a general guide for biodegradability.

Pathways of bacterial biodegradation of lower molecular weight PAHs (2-3 rings) have been studied extensively (Haritash \& Kaushik, 2009), but biodegradation pathways of higher molecular weight PAHs (4 or more rings), are not as well supported by research 
(Haritash \& Kaushik, 2009). There are far fewer organisms known that can use these larger molecules as carbon or energy sources.

The generalized pathways of bacterial and fungal PAH biodegradation are shown in Figure 2.4. In these catabolic pathways oxygen must be present to initiate a reaction with the PAH ring (Gibson et al., 1968). The hydroxylation of benzoid aromatics (aromatics containing benzene rings, which are especially stable) involves the integration of molecular oxygen (Gibson, 1984). Bacteria use dioxygenase enzymes to incorporate both oxygen atoms of molecular oxygen to form cis-dihydrodiols (Gibson et al., 1990). These compounds are selectively dehydrogenated by cis-dihydrodiol dehydrogenases (Patel \& Gibson, 1974). This process rearomatizes the benzene nucleus to form dihydroxylated intermediates. This is followed by either an ortho or meta fission by dioxygenases, with respect to the connected aromatic ring. This step is largely dependent on which dioxygenase is produced by the bacteria. For this reaction to occur, the benzene ring must have two hydroxyl groups ortho or para to each other. If this requirement is met, the benzene ring can be cleaved either between (intradiol fission) or adjacent (extradiol fission) to the hydroxyl groups (Cerniglia, 1992). The enzymes that perform this step are highly region- and stero-selective (Gibson, 1984). 


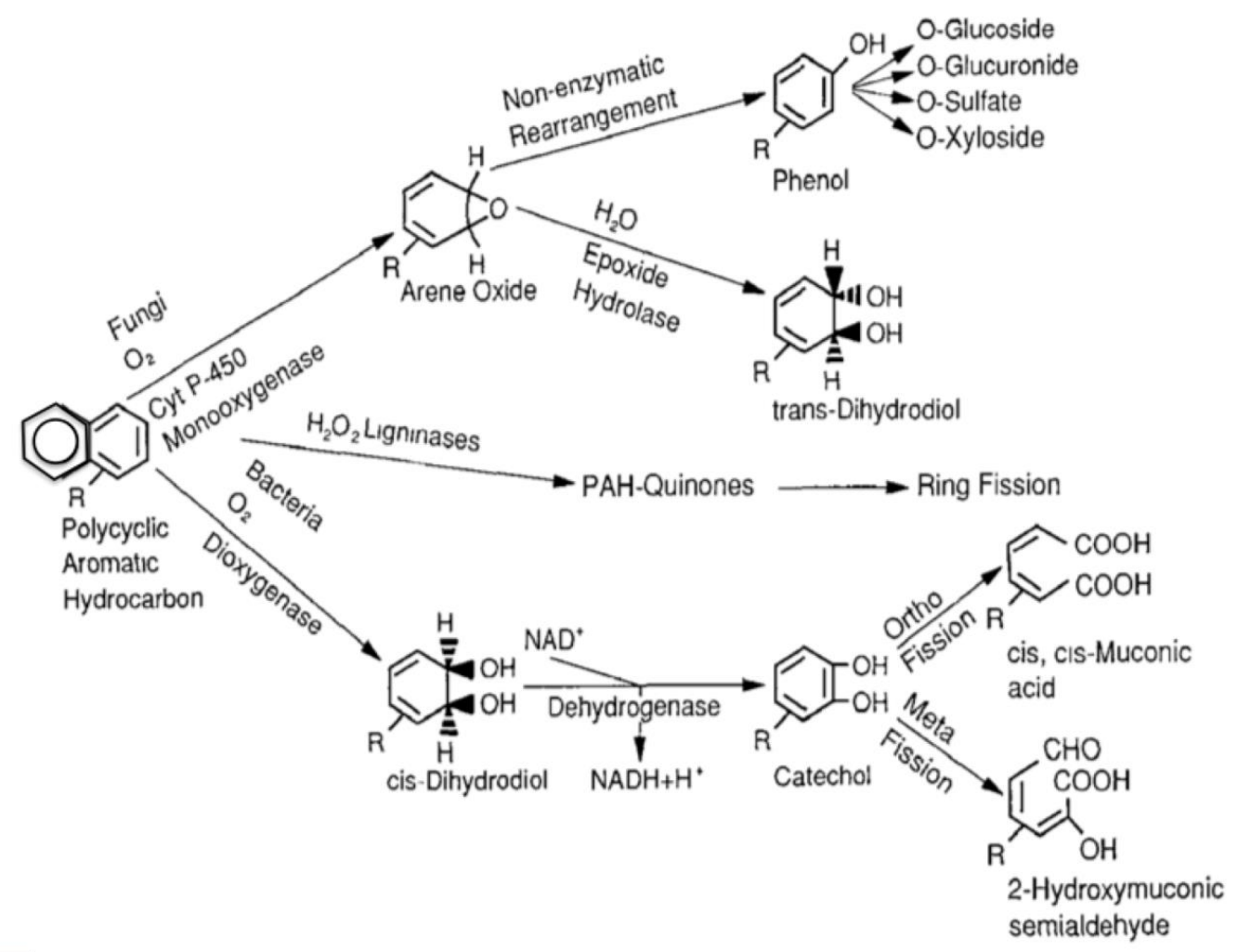

Figure 2.3: A summary of microbial and fungal catabolism of polycyclic aromatic hydrocarbons (Gibson et al., 1990). Many of the end products shown here are easily biodegraded further to $\mathrm{CO} 2$.

These processes can be repeated to breakdown large PAHs into smaller and smaller ones, yielding smaller, less-toxic molecules that are easier to breakdown by the natural soil microflora. However, the more rings in a PAH the less likely this process is to be initiated. 4 and 5 ring PAHs remain particularly resistant to biodegradation, due to their high resonance energy and low solubility in water (Cerniglia, 1992). It should also be noted that there has been relatively little research done on complex mixtures of PAHs, and most studies focus on single PAHs in order to minimize variables. However, PAHs 
often exist in complex mixtures in the environment, and this is certainly true in Area IV at SSFL.

\section{Fungal Biodegradation of PAHs}

A number of fungi species, both ligninolytic and non-ligninolytic, have been identified as being capable of PAH biodegradation (Tortella, Diez, and Durá 2005). Lingolytic enzymes include lignin peroxidase, laccase, and manganese peroxidase. They function by oxidizing carbon basecpolymers common in natural lignins, and these same enzymes can oxidize PAHs (Haritash and Kaushik 2009). Other enzymes involved include oxygenase and dehydrogenase, which are common catabolic enzymes (as described above). Fungi secrete these enzymes, and others, and digest molecules outside their cells then absorb the products of the enzymatic reactions for nutrients.

Ligninolytic fungi have also been shown to degrade PAHs using extracellular lignin peroxidases. These enzymes not only degrade lignin but also catalyze one-electron oxidations of PAHs to quinones (Haritash and Kaushik 2009). Lignin peroxidases are known to oxidize PAHs that have less than a $7.6 \mathrm{eV}$ ionization energy (Haemmerli et al., 1986). Those PAHs include pyrene, anthracene, coronene and others (Kuroda 1964).

The best studied non-ligninolytic fungus, Cunninghamella elegans, uses cytochrome P450 moonoxygenase to break down aromatic rings. The enzyme integrates 1 of the 2 oxygen atoms from molecular oxygen into the aromatic nucleus of a benzene ring. The remaining oxygen atom is used to form water (Laskin, 1984). The resulting structure is an arene oxide intermediate, which is further broken down by other pathways (Cerniglia, 1992). 


\subsubsection{Polychlorinated biphenyls (PCBs)}

\section{Physical Properties}

PCBs are chlorinated biphenyl compounds (Figure 2.5). Although the name is "polychlorinated", monochlorinated biphenyls are generally included under the name "PCBs". There can be between 1 and 10 chlorine atoms bonded to the biphenyl in 10 different positions, making 209 possible unique congeners. The number of chlorine atoms they contain often classifies PCB congeners. PCBs with the same number of chlorines are called homologs. The most common way of naming PCBs was created by Monsanto Corp., who produced PCBs commercially. This method names mixtures of PCBs with a 4-digit number, with the first 2 digits indicating the number of carbons (12) and the second 2 digits indication the chlorine content by weight percent. These mixtures were sold by Monsanto under the trade name Aroclor. For example, Aroclor 1254 is a mixture of PCBs that includes mono-through heptachloroinated homologs with a 54\% chlorine content by weight (ASTDR, 2010).

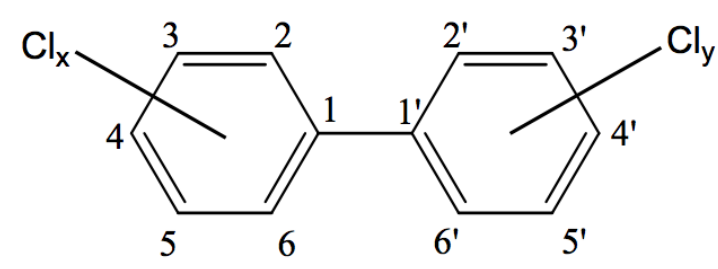

Figure 2.4: Generic Structure of PCBs (ASTDR 2010). Positions 2,2',6, and 6' are ortho positions, positions 3,3',5 and 5' are meta positions and positions 4 and 4' are para positions. 


\section{Toxicity of PCBs}

PCB contamination is a major health concern to both humans and wildlife for three main reasons: they are widespread due to the variety of applications they were used for, they are difficult to degrade and soluble in fatty tissue so they bioaccumulate, and they are toxic, carcinogenic, and mutagenic (Narquis, 2007). Studies also indicate that some PCBs can bind to receptors intended for estrogen or estradiol, making them potential endocrine disrupters (Wang et al., 2006). Bioaccumulation is also a major concern (Bernard et al., 2002). The half lives of PCBs in the human body are 7-10 years (Wang et al., 2006), which allows time for accumulation.

Because each congener has a different toxicity and PCBs are usually in mixtures, the toxicity of PCB congeners is measured as toxic equivalents (TEQ), which are calculated using toxic equivalency factors (TEFs) for each congener (Narquis, 2007). The TEF values of each PCB congeners are multiplied by the concentration of each congener in a sample to calculate the TEQ. These TEQ values are used in risk assessments and regulatory control (Van den Berg et al., 1998).

The PCBs measured in Area IV were quantitated based on the closest Aroclor product match to the congener concentrations measured. The PCBs analyzed in Area IV most closely match the congener make-up of Aroclors 1248, 1254, and 1260. The composition and physical properties including solubility, vapor pressure, Log Kow, molecular weight, and congener compositions of these PCB mixtures are detailed in Heidelore Fiedler, (1997) and ASTDR, (2010). 


\section{Abiotic Weathering of PCBs}

Due to the extremely low vapor pressure of PCBs, significant volatilization from soils is unlikely. PCB volatilization has been reported in measurable quantities at temperatures of $109^{\circ} \mathrm{C}$ (Dubey \& Dugal, 1977), but soil temperatures at SSFL are unlikely to exceed $30^{\circ}$ C. However, some PCB volatilization is likely to have occurred during the 2005 wildfire at the site.

PCBs in contaminated soil are likely to remain stationary in the organic fraction of the soil because of the chemical properties of PCBs, most notably their low solubility in water and high octanol-water partition coefficients (Davis \& Wade, 2003). This sequestration in the soil may lead to reduced bioavailability and therefore low biodegradation rates of PCBs in soil (Hyun et al., 2010). Abiotic degradation processes, such as photo-oxidation and chemical degradation, are expected to be negligible for this site (Sinkkonen \& Paasivirta, 2000) because photo-oxidation typically only affects degradation rates in aquatic environments.

\section{Biodegradation of PCBs}

Both bacteria and fungi have been shown to biodegrade complex PCB mixtures (Abraham et al. 2002; Čvančarová et al. 2012). Bacterial degradation of PCBs typically occurs via reductive dechlorination of highly chlorinated PCB congeners under anaerobic conditions (Quensen et al., 1990) followed by aerobic biodegradation of the lightly chlorinated congeners by bacteria using these compounds as a carbon source (Haggblom et al., 2012). These two pathways combined can completely mineralize PCB mixtures. However, this multiple-step process is complex, involving multiple microorganisms, and 
tends to be slow (Seeger et al., 1997). Additionally, aerobic microbes are fairly selective towards lower chlorinated PCBs, often leaving the higher chlorinated congeners untouched (Pieper, 2004).

\section{Bacterial Biodegradation of PCBs: Anaerobic Reductive Dechlorination of PCBs}

Anaerobic dechlorination is a process that uses a hydrogen atom to displace chlorine atoms on the PCB molecule, thus yielding a lower chlorinated congener. This process is typically cometabolic, involving an electron donor which must be available to the bacteria (Quensen et al., 1988). The rate of anaerobic dechlorination decreases as the degree of chlorination increases. The microbial population also has a large impact on which PCBs can be dechlorinated, and how fast (Quensen et al., 1990).

Anaerobic dechlorination does not work equally well for all positions of chlorine on the PCB molecule. Typically, meta and para chlorines are preferentially dechlorinated, while ortho chlorines are more recalcitrant. A study conducted by Quensen III et al. (1990), used microbial populations in sediment from the Hudson River, which has been highly contaminated with PCBs for decades, to reductively dechlorinate Aroclor 1242, 1248, 1254, and 1260. After 25 weeks, chlorines in meta and para positions showed significant dechlorination for all of these Aroclors except 1260. Aroclors 1242, 1248, and 1254 showed 85,75 , and $63 \%$ removal of chlorines from meta and para positions. Ortho chlorines remained largely unaffected during the 25 -week study. The experiment was repeated using sediments from Silver Lake, Massachusetts, and this time showed a 19\% decrease in meta and para chlorines removed (Quensen et al., 1990). However, 
dechlorination of ortho chlorines was observed for single congeners supplemented with fatty acids (Wiegel \& Wu, 2000).

Numerous researchers have isolated bacteria that can reductively dechlorinate PCBs in an anaerobic environment. A summary of the results of these studies is presented in the next section (Section 3). Rates measured in these laboratory experiments varied from $1 \%$ in 3 days to $98 \%$ in 48 hours. However it should be noted that these were controlled laboratory experiments that usually had high concentrations of PCBs spiked into the soil. Rates measured in the field are not expected to be accurately predicted by these values. It is important to understand that these rates were determined in the conditions stated in these studies, and that varying physical conditions can have a large impact on rates as well (Tiedje et al., 1991).

Physical and chemical factors can dictate the rate of dechlorination by limiting the microbial growth or the ability of the microbes to uptake and react with the PCBs. PCB concentration is one such factor. The optimum PCB concentration range for dechlorination is between 200 to $1000 \mathrm{ppm}$ (w/w) in sediment (Quensen et al., 1988). Below 50 ppm dechlorination is reported to be halted (Tiedje et al., 1993). Bioavailability of PCBs in the environmental matrix is also a key factor. If PCBs are dissolved in organic phases within the soil or covered by a layer of organic matter the microbes or extracellular enzymes could not easily access them. Temperature and climate can also affect PCB biodegradation rates. For example, dechlorination of a lower chlorinated PCB mixture (Aroclor 1242) was shown to occur at $25^{\circ} \mathrm{C}$, but not at $37^{\circ} \mathrm{C}(\mathrm{Wu}$, Bedard, and Wiegel 1996). Effects of temperature are also apparent in the studies on Woods Pond sediment, performed by Wu, Q.; Bedard, D.L.; and Wiegel, J (Q. Wu et al., 1996; Q. Wu, 
Bedard, \& Wiegel, 1997a, 1997b). The temperature that the microcosm was held at (between $4^{\circ}$ and $66^{\circ} \mathrm{C}$ ) dictated which pathway the microbes used to dechlorinate the PCB mixture.

If a population is starved of electron acceptors or carbon sources it will slow the rate of biodegradation. It has been shown that adding carbon sources to anaerobic soil can increase the rate of dechlorination (Nies \& Vogel, 1990). Nitrogen and phosphorous are also essential nutrients for bacteria, and are used for both biomass production and metabolism(Thirukkumaran \& Parkinson, 2000). If a soil is low in either nutrient, it will hinder biodegradation rate through one or both of those mechanisms. Finally, inhibitors and other contaminants should be considered as potential rate limiting agents. Metals, oils, grease, and solvents are sometimes toxic to microbial populations and may have an effect on their ability to dechlorinate (Tiedje et al., 1993). It has been found that high concentrations of oil and grease are associated with lower dechlorination rates (Tiedje et al., 1993).

\section{Aerobic Pathways of Bacterial PCB Biodegradation}

Following anaerobic dechlorination, aerobic bacteria can break down some of the remaining dechlorinated or lightly chlorinated biphenyls. Reported aerobic pathways all start with biphenyl 2,3-dioxygenases (Pieper, 2004). This is known as the upper, or bph pathway. This pathway may be initiated on PCBs that contain low amounts of chlorine (1-2 chlorines), but this depends on the degree and position of chlorination as well as the specificity of these 2,3-dioxygenases (Pieper, 2004). Although some bacteria possess and use this family of enzymes, PCBs are rarely used as carbon or energy sources themselves. 
Their degradation is usually part of some cometabolism process, and therefore the upper pathway often produces metabolites that are dead-end products (Bedard \& Haberl, 1990; Furukawa et al., 1979; and Seeger et al., 1997).

Although some bacterial species have been observed using these enzymes to degrade lower chlorinated biphenyls, it is not always possible. Some of the enzymes in the pathway may not be able to use the chlorinated product of the previous reaction, creating “dead-end" products including dihydrodiols (Brühlmann \& Chen, 1999), dihydroxybiphenyl (including 3,4-dihydroxylated derivatives) (Tř́iska et al., 2004), or chlorinated HOPDAs (Furukawa et al., 1979; Seeger et al., 1997). Although it is difficult, it is not impossible for these to be degraded. It is rare for a bacterium to express all the correct variations of these enzymes to be able to degrade a lower chlorinated PCB all the way via this pathway (Pieper, 2004 and Seeger et al., 1997).

\section{Fungal Biodegradation of PCBs}

Fungal biodegradation of PCBs is typically mediated by species like white-rot fungi which produce ligninolytic enzymes which are used to break down the complex organic molecules in lignin (Čvančarová et al. 2012; Eaton 1985; Thomas, Carswell, and Georgiou 1992; Novotný et al. 2004; Yin et al. 2011). These enzymes have a broad specificity, and have been reported to biodegrade PCB congeners with 1 to 6 chlorines (Čvančarová et al., 2012). By far the most commonly studied ligninolytic fungi for biodegradation of PCBs (as well as many other contaminants) is white-rot fungi (Phanerochaete sp.). Some pathways for PCB biodegradation by this fungi have been proposed by Čvančarová et al., 2012. The degradation products revealed that the various 
PCB congeners were most likely being transformed through a reductive pathway, producing chlorobenzoates (Čvančarová et al., 2012). As stated above, these chlorobenzoates are difficult for bacteria to breakdown, and thus are often "dead-end" products of bacterial biodegradation of PCBs (Pieper, 2004). However, ligninolytic fungi can degrade these further to produce even less toxic products (Čvančarová et al., 2012). The key to the fungi's success is the extracellular enzymes that they secrete with low substrate specificity.

There are 4 key groups of enzymes used to mediate PCB biodegradation by fungi: lignin peroxidase (LiP), manganese peroxidase (MnP), versatile peroxidases (VP) and laccases, which belong to the phenol oxidase family (Čvančarová et al., 2012). These enzymes are non-specific enough that they will react with a wide range of congeners, in great contrast to bacterial PCB degradation. Several studies suggest reducing PCB concentrations can improve the rate of degradation (about $200 \mathrm{ppm}$ for dichloro congeners, and $1 \mathrm{ppm}$ for hexachloro congeners) (Yin et al., 2011). Phanerochaete chrysosporium, Trametes versicolor and Pleurotus ostreatus are some of the most common ligninolytic fungal degraders reported for PCBs (Novotný et al., 2004).

\subsubsection{Dioxins (polychlorinated dibenzo-p-dioxins and dibenzofurans)}

\section{Physical Properties and Toxicity of Dioxins}

Chlorinated dioxins are comprised of two families of compounds, which are tricyclic, planar, and aromatic. Polychlorinated dibenzo-p-dioxins (PCDD) have a backbone of two benzene rings connected with two ether linkages and have chlorination possible at 10 different sites, resulting in 75 possible congeners. Polychlorinated dibenzofurans (PCDF) 
are connected with only one ether linkage and have 9 possible chlorination sites, resulting in 135 possible congeners (because of the reduced symmetry of the PCDF backbone, there are more unique congeners than for PCDD). See Figure 2.5 for the general structures of PCDD, PCDF, dioxins, and dibenzofuran. Physical properties of important PCDDs and PCDFs, including vapor pressure of subcooled liquid $\left[\mathrm{P}_{\mathrm{L}}\right]$, water solubility [S], octanol-water partitioning coefficient $\left[\log K_{o w}\right]$ and reported half-lives $\left(t_{1 / 2}\right)$ can be found at Haglund 2007a. Major sources of dioxin (including PCDDs and PCDFs) as unwanted byproducts are of industrial paper production, herbicide and pesticide synthesis, metal smelting, and waste incineration, (Tuppurainen et al., 2003). In particular, incineration of chlorinated waste has been shown to yield dioxins in quantities of concern for public health (Brzuzy and Hites 1996). Natural burning processes such as forest fires and volcanic activity can also produce these compounds.

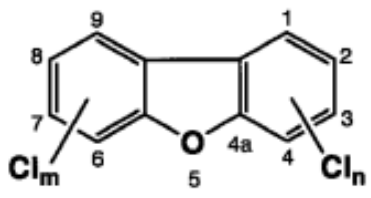

Polychlorinated dibenzofurans (PCDF)

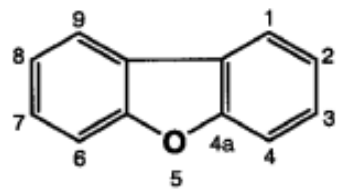

Dibenzofuran (DF)

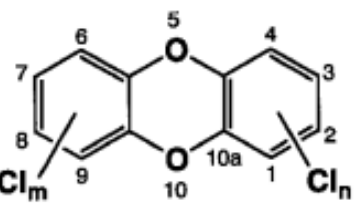

Polychlorinated dibenzo-p-dioxins (PCDDs)

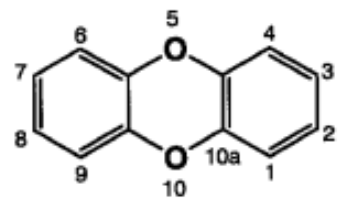

Dibenzo-p-dioxin (DD)

Figure 2.5: Chemical structures of Dibenzo-p-dioxins (DD) and dibenzofurans (DF), showing analogues and numbering conventions (Nojiri et al., 2001). 
Dioxins are highly hydrophobic. There are 17 PCDDs having a 2,3,7,8 substitution of chlorination, which results in the most toxicity to higher organisms (Van den Berg, Birnbaum, Bosveld, Brunstrom, et al. 1998; Boening 1998). PCDDs and PCDFs are of environmental concern due to their well-documented effects on the human endocrine, immune, and reproductive systems (Panteleyev and Bickers 2006; Consonni et al. 2008; Turyk, Anderson, and Persky 2007) as well as their dermal toxicity and carcinogenicity (Van den Berg et al. 1998). Of the 75 congeners of PCDDs and 135 congeners of PCDFs, 30 are considered significant toxins (Peng et al., 2013). When dioxins are formed in the environment, a number of different congeners are formed, all with different toxicities (Van den Berg et al. 1998). To collectively quantify the toxicity of dioxin mixtures, the toxic equivalency (TEQ) was developed which provides an estimate of the overall toxicity of such mixtures based on the concentration of each congener in the environmental medium (e.g. soil, air or water). To calculate the TEQ, toxic equivalency factors (TEFs) are used for each congener which is a measure of that congener's toxicity relative to the most toxic congeners. In this system, the most toxic congeners $(2,3,7,8$ TCDD) are assigned a TEF of one, and all other congeners have been assigned a number proportional to their toxicity (Table 2.4). For mixtures of congeners the TEQ is calculated by multiplying each individual concentration by the TEF for that congener and then summing these products for all congeners. 
Table 2.4: Dioxin toxic equivalency factors (TEFs) reported by the World Health Organization (WHO)

\begin{tabular}{|c|c|c|}
\hline $\begin{array}{c}\text { Group of } \\
\text { Compounds }\end{array}$ & Name of Compound & WHO TEF \\
\hline \multirow[t]{7}{*}{ Dioxins } & 2,3,7,8-Tetra-CDD & 1 \\
\hline & 1,2,3,7,8-Penta-CDD & 1 \\
\hline & 1,2,3,4,7,8-Hexa-CDD & 0.1 \\
\hline & $1,2,3,6,7,8$-Hexa-CDD & 0.1 \\
\hline & $1,2,3,7,8,9$-Hexa-CDD & 0.1 \\
\hline & 1,2,3,4,6,7,8-Hepta-CDD & 0.01 \\
\hline & OCDD & 0.0001 \\
\hline \multirow[t]{10}{*}{ Dibenzofurans } & 2,3,7,8-Tetra-CDF & 0.1 \\
\hline & $1,2,3,7,8$-Penta-CDF & 0.05 \\
\hline & 2,3,4,7,8-Penta-CDF & 0.5 \\
\hline & $1,2,3,4,7,8-\mathrm{Hexa}-\mathrm{CDF}$ & 0.1 \\
\hline & $1,2,3,6,7,8-$-Hexa-CDF & 0.1 \\
\hline & $1,2,3,7,8,9-\mathrm{Hexa}-\mathrm{CDF}$ & 0.1 \\
\hline & $2,3,4,6,7,8-\mathrm{Hexa}-\mathrm{CDF}$ & 0.1 \\
\hline & 1,2,3,4,6,7,8-Hepta-CDF & 0.01 \\
\hline & 1,2,3,4,7,8,9-Hepta-CDF & 0.01 \\
\hline & OCDF & 0.0001 \\
\hline \multirow[t]{4}{*}{ Coplanar } & $3,3^{\prime}, 4,4^{\prime}-\mathrm{TCB}(77)$ & 0.0001 \\
\hline & $3,4,4^{\prime}, 5-\mathrm{TCB}(81)$ & 0.0001 \\
\hline & $3,3^{\prime}, 4,4^{\prime}, 5-\mathrm{PeCB}(126)$ & 0.1 \\
\hline & $3,3^{\prime}, 4,4^{\prime}, 5,5^{\prime}-\mathrm{HxCB}(169)$ & 0.01 \\
\hline \multirow[t]{7}{*}{ Mono-ortho- } & $2,3,3^{\prime}, 4,4^{\prime}-\mathrm{PeCB}(105)$ & 0.0001 \\
\hline & 2,3,4,4',5-PeCB (114) & 0.0005 \\
\hline & $2,3^{\prime}, 4,4^{\prime}, 5-\mathrm{PeCB}(118)$ & 0.0001 \\
\hline & $2^{\prime}, 3,4,4^{\prime}, 5-\mathrm{PeCB}(123)$ & 0.0001 \\
\hline & $2,3,3^{\prime}, 4,4^{\prime}, 5-\mathrm{HxCB}(156)$ & 0.0005 \\
\hline & $2,3,3^{\prime}, 4,4^{\prime}, 5^{\prime}-\mathrm{HxCB}(157)$ & 0.0005 \\
\hline & $2,3^{\prime}, 4,4^{\prime}, 5,5^{\prime}-\mathrm{HxCB}(167)$ & 0.00001 \\
\hline
\end{tabular}

\section{Abiotic Weathering Effects on Dioxins}

Abiotic processes which could potentially contribute to weathering and natural attenuation of dioxins include volatilization, photo-oxidation and sequestration in soil (Vasquez, Regens, and Gunter 2004). Volatilization of dioxins from soil is a potential 
pathway for natural attenuation, but volatilization rates are reported to be very slow (Trapp and Matthies 1997), as expected from the low vapor pressure of dioxins. For highly contaminated soils, dioxins may be volatilized from soils and deposited onto plant tissue (Trapp and Matthies 1997), but this is not likely to be significant at SSFL because the concentrations of dioxins are relatively low.

Early studies suggested that chlorinated dioxins are not photodegraded in soils (Isensee and Jones 1975), but later studies implicated ultraviolet radiation in the photodegredation of dioxins, accounting for up to $10 \%$ of degradation rates observed in the field. However, this UV effect was only observed in the top 5-6 $\mathrm{mm}$ of the soil as UV light penetration is blocked in the deeper soils (Kieatiwong et al., 1990). Another study estimated that most of the photolytic activity occurs at soil depths of only 0.06-0.13 mm (Miller et al. 1989).

Dioxins have long been considered to be immobile in soils (Isensee and Jones 1975) due to their adsorption to organic material in soils. This adsorption leads to sequestration in the soil matrix which greatly reduces bioavailability of these compounds to biodegradation (Cornelissen et al. 2005). After deposition on soil, the pollutants will be redistributed from weak adsorption sites to stronger, from the surface to the interior of the soil, and even further into the finer pores. The contaminants become very recalcitrant to biodegradation in soils with finer soil particles such as clay and silt. On the positive side, this reduction in bioavailability could also lead to decreased toxicity (Alexander, 1995). Also, the hydrophobic nature of dioxins limits their solubility in water and thus contamination of groundwater and rainwater runoff do not pose serious threats. 


\section{Biodegradation of Dioxins}

Biodegradation of dioxins, as described below, typically leads to a reduction of concentrations of easily degraded components of dioxin mixtures, leaving the more recalcitrant fractions and more sequestered components to persist in the soil. This weathering process leads to a decrease in dioxin concentrations and toxicity over time, which eventually reaches a lower limit (Hatzinger \& Alexander, 1995).

PCDDs, PCDFs, and other dioxin-like chemicals are part of the natural chlorine cycle, which leads to their eventual biodegradation in the environment by either bacteria or fungi. Similar to PCBs, dioxins with fewer chlorine substitutions are typically biodegraded aerobically by bacteria, while chlorinated dioxins with more than four chlorines are not known to be biodegraded by aerobic bacteria (Sakaki \& Munetsuna, 2010). However, these higher chlorinated dioxins can be reductively dechlorinated by anaerobic bacteria, resulting in their transformation to lower chlorinated dioxins which are then amenable to aerobic biodegradation (Sakaki et al. 2010). Thus, bacterial biodegradation of dioxins may be mediated by a complex consortium of bacteria in anaerobic and aerobic zones of sediments or soils. The higher chlorinated compounds tend to persist longer in the environment due to decreased rates of degradation, with halflives reported from 12 to 170 years (Kjeller \& Rappe, 1995). Bacterially-mediated anaerobic dechlorination and aerobic biodegradation are described separately below in Sections 6.3.1 and 6.3.2, respectively. 


\section{Bacterial Anaerobic Reductive Dechlorination of Dioxins}

Many researchers have demonstrated the ability of microorganisms to dechlorinate dioxins through the process of reductive dehalogenation (Ballerstedt et al., 2004; Bunge et al., 2003). Dehalococcoides has been the most extensively studied genus of bacteria with the ability to reductively dechlorinate dioxins. These bacteria grow under anaerobic conditions, using multiple dehalogenase enzymes to remove chlorine from dioxin congeners through a cometabolic process which requires an external electron donor such as sugars, hydrogen or lactate. Successful dechlorination of higher chlorinated compounds may depend on consortia of different microorganisms rather than a single species (Beurskens et al. 1995; Wittich et al. 1999; Pelz et al. 1999; Bunge et al. 2008).

Anaerobic dechlorination of 1234-tetrachloro dibenzo-p-dioxin (TCDD) has been demonstrated by Dehalococcoides sp. CBDB1 (Bunge et al., 2003) and Dehalococcoides ethenogenesstrain 195 (Fennell et al., 2004). Both bacterial species produce the same end

product, 2-chloro dibenzo-p-dioxin, which can then be further metabolized aerobically by different microorganisms.

\section{Bacterial Aerobic Mechanisms for Degradation of Lower Chlorinated Dioxins}

Aerobic biodegradation of lower chlorinated dioxins, dibenzo-furans, and dibenzodioxins occurs via many pathways, which can lead to a wide range of intermediates and products. Biodegradation of these lightly chlorinated dioxins has been documented for bacteria in the genera of Sphingomonas, Pseudomonas and Burkholderia (Nam et al., 2006). The two major enzyme systems used by microorganisms to degrade lower chlorinated contaminants are dioxygenase and cytochrome P450 (Sakaki \& Munetsuna, 
2010). Dioxygenase enzyme pathways for aerobic dioxin biodegradation explained in detail by (Field and Sierra-Alvarez 2008). The complete metabolism of dioxin like compounds may be dependent on several different microbial populations to degrade the metabolites produced by biodegradation (Field and Sierra-Alvarez 2008; Arfmann, Timmis, and Wittich 1997; Bunge et al. 2008; Holliger et al. 1992).

\section{Fungal Biodegradation of Dioxins: White-rot fungi}

Ligninolytic fungi have also been shown to biodegrade dioxins. The lignin-degrading peroxidases secreted by fungi such as white-rot fungi have been shown to cometabolically biodegrade dioxins (Field and Sierra-Alvarez 2008). White rot fungi have been shown to have promising rates of biodegradation of PCDDs in numerous studies (Takada et al. 1996a). White-rot fungi have developed complex enzyme systems to degrade lignins, and these same enzyme systems can biodegrade many ordinarily recalcitrant compounds. Phanerochaete chrysosporium, the most studied species of the white rot fungi, has been shown to biodegrade several dioxins (Bumpus et al., 1985 and Valli et al., 1992) including highly chlorinated dioxins (Takada, Nakamura, Matsueda, Kondo, \& Sakai, 1996b) as well as chlorinated DD (Joshi \& Gold, 1994). In addition to P. chrysosporium, several other fungal species have been shown to biodegrade dioxins. Coprinellus spp., Phlebia lindtneri, Pseudallescheria boydii, and Cordyceps sinensis, along with other fungi have been associated with effective biodegradation of PCDDs and dioxin metabolites (Ishii et al. 2009; Kamei, Suhara, and Kondo 2005; Nakamiya et al. 2005; Suhara et al. 2011). 
Fungal dioxin biodegradation is mediated by a number of complex enzyme-mediated mechanisms, which are described only briefly here. More detailed descriptions of fungal biodegradation mechanisms are available in the literature (Bumpus et al., 1985) (Sakaki \& Munetsuna, 2010) (Valli et al., 1992). P. chrysosporium has been shown to secrete two heme peroxidases, lignin peroxidase (LiP) and manganese-dependent peroxidase (MnP). The LiP can also degrade various environmental pollutants including PCDDs (Sakaki \& Munetsuna, 2010). MnP can only react with these molecules after the cleavage of the dioxin ring (Valli et al., 1992). White-rot fungi may also mediate dioxin biodegradation using cytochrome P450 enzymes. It has also been shown that $P$. chrysosporium possesses 148 CYP genes, which suggests that cytochrome P450 enzymes may be involved in these processes (Kasai et al., 2010).

\subsection{TRFLP and qPCR Background}

\section{TRFLP Background}

TRFLP analysis is a method to characterize microbial communities without culturing the microorganisms. DNA is first extracted from the soil or other medium and then the DNA is amplified using polymerase chain reaction (PCR) using primers for the types of organisms to be characterized (16S for bacteria and ITS for fungi). The amplified DNA is cut with restriction enzymes resulting in fragments of DNA, and the lengths of the fragments are determined using chromatography. Different microorganisms have different $16 \mathrm{~S}$ or ITS sequences, and will therefore be cut in different places producing various fragment lengths. The pattern of resulting fragment lengths can be used to characterize the microbial diversity of the sample and compare community composition between samples. A large number of different peaks suggests high diversity, while few 
peaks suggests low diversity. Comparison of observed TRFLP patterns to libraries of TRFLP patterns for known microorganisms can be used to infer the presence of certain types of microorganisms.

\section{qPCR Background}

Quantative PCR, or sometimes real-time PCR, is known as qPCR. It is a molecular biology method that used PCR to amplify specific gene targets and quantifying the number of copies produced in real time (Wilhelm and Pingoud 2003). Quantified DNA can be measured in total copies or in relative amounts. qPCR can also be used for simple detection of a gene target. The process uses the standard PCR methodology, but includes a marker that is used to quantify the DNA as it is copied. This marker can be either a nonspecific fluorescent dye that binds to any double-stranded DNA or a sequencespecific DNA probe consisting of short, single-stranded DNA sequences that are labeled with a fluorescent molecule. These DNA probes get integrated into the complementary sequence so that messenger RNA (mRNA) can be quantified (Udvardi, Czechowski, and Scheible 2008; Sigma Aldrich 2012). Microbial Insights® (MI) is a well established company that offers qPCR services. Another service they offer is the QuantArray® Petro. The assay is a new technology that combines the parallel nature of DNA microarrays and the accuracy qPCR to quickly and precisely detect a number of gene targets all at once. This assay was developed by MI to quantify key organisms, important functional genes, and terminal electron acceptor processes for a particular application simultaneously and economically. In the case of the QuantArray® Petro assay, this application is the environmental remediation of soils contaminated with hydrocarbons (PHCs, BTEX, and PAHs) (Microbial Insights 2012). 
3.0 Natural Attenuation Rates and Microbial Degraders Reported in the Literature The literature review yielded a large amount of information regarding the biodegradation of the COIs, both qualitatively in terms of natural attenuation rates from both field and laboratory studies and quantitatively in terms of known degraders for all four COI groups. The reported natural attenuation rates can be used to gauge approximately how long it will take to remediate SSFL soil. The lists of known degraders were also used in conjunction with TRFLP and 16S sequencing from culturing experiments results to determine if the microbes on the site have the ability to biodegrade these COIs.

\subsection{Reported Rates of Natural Attenuation of Petroleum Hydrocarbons (non-PAH)}

Biodegradation rates of TPHs span a wide range and have been shown to decrease in the following general order: saturates $>$ light aromatics $>$ high-molecular-weight aromatics $>$ polar compounds (Leahy \& Colwell, 1990). These rates can be affected by multiple biological, physical, and chemical factors. Based on a thorough literature review, unamended first-order biodegradation rate constants range from approximately $3.8 \times 10^{-4}$ to $3.3 \times 10^{-2}$ day $^{-1}$ in field studies and $8.1 \times 10^{-4}$ to 0.27 in lab studies (Table 3.1). Since TPH biodegradation rates have been reported in over 100 publications, the rates reported in Table 3.1 are only those reported in the most cited papers.

Rates observed in laboratory studies are often higher than rates observed in the field (Table 3.1) and there are a number of possible reasons for this. Compared to some lab studies which spike fresh contaminants into soil, contaminants in the field are more weathered, leaving the more recalcitrant compounds. Contaminants in the field may also be sequestered in the soil matrix and less bioavailable (Osuji, Udoetok, and Ogali 2006). 
An important consideration is that petroleum hydrocarbon contamination is comprised of thousands of different compounds, each with its own biodegradation kinetics (Abalos et al. 2004). Biodegradation of some compounds is more complete than others, and some compounds are more recalcitrant than others (Leahy \& Colwell, 1990). For this reason, biodegradation may follow first-order kinetics during initial biodegradation, followed by much slower biodegradation of the more recalcitrant, sequestered compounds. This hindered kinetics is sometimes referred to as "hockey stick kinetics" because of the modified shape of the concentration vs. time curve (Dados et al. 2014). Thus, hydrocarbon-contaminated sites are often left with some residual contamination, which is recalcitrant and not very bioavailable. 
Table 3.1: Biodegradation rates of petroleum hydrocarbons reported in the

literature (most cited field and laboratory studies). (PHC: Petroleum Hydrocarbon)

\begin{tabular}{|c|c|c|c|c|c|c|c|c|}
\hline \multirow{2}{*}{ Contaminant } & \multirow{2}{*}{ Matrix } & \multirow{2}{*}{ Lab/Field } & \multicolumn{2}{|c|}{$\begin{array}{c}\text { Soil TPH or PHC } \\
\text { Concentration }(\mathrm{mg} / \mathrm{kg})\end{array}$} & \multirow{2}{*}{$\begin{array}{l}\text { Length of } \\
\text { Study } \\
\text { (days) }\end{array}$} & \multicolumn{2}{|c|}{ Biodegradation Metric } & \multirow{2}{*}{ Reference } \\
\hline & & & Initial & Final & & $\begin{array}{l}\text { Percent } \\
\text { Degraded }\end{array}$ & $\begin{array}{c}\text { Rate of } \\
\text { Degradation } \\
\text { (mg/kg/day) }\end{array}$ & \\
\hline Diesel & Soil & Lab & 10,000 & 3,550 & 30 & $65 \%$ & 215 & Namkoong et al., 2002 \\
\hline Octane & Soil & Lab & 700 & 691.6 & 15 & $1.20 \%$ & 0.56 & Moldes et al. 2011 \\
\hline Octane & Soil & $\mathrm{Lab}$ & 700,000 & 532,000 & 15 & $24 \%$ & 1,100 & Moldes et al., 2011 \\
\hline PHC & Soil & Lab & 2,815 & 1,439 & 60 & $48 \%$ & 22.9 & Llado et al., 2013 \\
\hline PHC & Soil & $\mathrm{Lab}$ & 2,985 & 788 & 160 & $74 \%$ & 13.7 & Li et al. 2006 \\
\hline PHC & Soil & Lab & 21,100 & 8,229 & 210 & $61 \%$ & 61.3 & Tang et al., 2012 \\
\hline PHC & Soil & Lab & 11,533 & 7,496 & 270 & $35 \%$ & 15 & Couto et al., 2010 \\
\hline PHC & Soil & Lab & 8,378 & 1,608 & 112 & $81 \%$ & 60 & Baek et al., 2007 \\
\hline PHC & Sludge & Lab & 48,800 & 20,984 & 365 & $57 \%$ & 76 & Hutchinson et al., 2001 \\
\hline $\mathrm{PHC}$ & Soil & $\mathrm{Lab}$ & 4,000 & 624 & 7 & $84.40 \%$ & 482 & Sarkar et al., 2005 \\
\hline PHC & Soil & Lab & 99.2 & 82.5 & 120 & $16.80 \%$ & 0.14 & Mishra et al., 2001 \\
\hline PHC & Soil & $\mathrm{Lab}$ & 11,975 & 5361 & 270 & $55 \%$ & 25 & Sabaté et al., 2004 \\
\hline $\begin{array}{l}\text { TPH }(\mathrm{C} 10- \\
\text { C32) }\end{array}$ & Soil & Field & 2,440 & 952 & 168 & $61 \%$ & 8.9 & Kaplan and Kitts, 2004 \\
\hline $\begin{array}{l}\text { TPH }(\mathrm{C} 12- \\
\mathrm{C} 23)\end{array}$ & Soil & Lab & 2,800 & $1,436.40$ & 84 & $48.70 \%$ & 16 & Bento et al., 2005 \\
\hline
\end{tabular}




\begin{tabular}{|c|c|c|c|c|c|c|c|c|}
\hline \multirow{2}{*}{ Contaminant } & \multirow{2}{*}{ Matrix } & \multirow{2}{*}{ Lab/Field } & \multicolumn{2}{|c|}{$\begin{array}{c}\text { Soil TPH or PHC } \\
\text { Concentration }(\mathrm{mg} / \mathrm{kg})\end{array}$} & \multirow{2}{*}{$\begin{array}{c}\text { Length of } \\
\text { Study } \\
\text { (days) }\end{array}$} & \multicolumn{2}{|c|}{ Biodegradation Metric } & \multirow{2}{*}{ Reference } \\
\hline & & & Initial & Final & & $\begin{array}{l}\text { Percent } \\
\text { Degraded }\end{array}$ & $\begin{array}{c}\text { Rate of } \\
\text { Degradation } \\
\text { (mg/kg/day) }\end{array}$ & \\
\hline TPH (C12- & Soil & $\mathrm{Lab}$ & 3,300 & 2,531 & 84 & $23.30 \%$ & 9.2 & Bento et al., 2005 \\
\hline $\begin{array}{l}\text { TPH }(\mathrm{C} 23- \\
\text { C40) }\end{array}$ & Soil & $\mathrm{Lab}$ & 9,450 & 5,131 & 84 & $45.70 \%$ & 51 & Bento et al., 2005 \\
\hline $\begin{array}{c}\text { TPH }(\mathrm{C} 23- \\
\mathrm{C} 40)\end{array}$ & Soil & $\mathrm{Lab}$ & 7,450 & 6,891 & 84 & $7.50 \%$ & 6.65 & Bento et al., 2005 \\
\hline $\mathrm{PHC}$ & Soil & Field & 14,000 & 12,200 & 365 & $12.80 \%$ & 4.9 & Balba et al., 1998 \\
\hline $\mathrm{PHC}$ & Soil & Field & 100,000 & 33,000 & 210 & $77 \%$ & 320 & Rhykerd et al., 1999 \\
\hline PHC & Soil & Field & 9,500 & 3,750 & 630 & $60 \%$ & 9.2 & Nedunuri et al., 2000 \\
\hline PHC & Soil & Field & 72,000 & 42,000 & 390 & $42 \%$ & 77 & Euliss et al., 2008 \\
\hline $\begin{array}{l}\text { TPH }(\mathrm{C} 10- \\
\text { C40) }\end{array}$ & Soil & Field & 9,000 & 7,164 & 7 & $20.40 \%$ & 260 & Lai et al., 2009 \\
\hline $\begin{array}{l}\text { TPH }(\mathrm{C} 10- \\
\mathrm{C} 40)\end{array}$ & Soil & Field & 3,000 & 2,838 & 7 & $5.40 \%$ & 23 & Lai et al., 2009 \\
\hline Crude oil & Soil & Field & 9,500 & 8,265 & 28 & $13 \%$ & 44 & Schaefer and Juliane, 2007 \\
\hline Crude oil & Soil & Field & 5,000 & 4,625 & 28 & $7.50 \%$ & 13 & Schaefer and Juliane, 2007 \\
\hline PHC & Soil & Lab & 60,600 & 57,570 & 35 & $5 \%$ & 87 & Mancera-López et al., 2008 \\
\hline PHC & Soil & Field & 7,000 & 7,448 & 730 & $-6.40 \%$ & -0.61 & Phillips et al. 2009 \\
\hline $\mathrm{PHC}$ & Soil & Field & 99,300 & 22,900 & 180 & $77 \%$ & 424 & Rojas-Avelizapa et al., 2007 \\
\hline
\end{tabular}




\subsection{Reported Rates of Natural Attenuation of PAHs}

A wide range of PAH biodegradation rates have been reported in the literature, and these are based almost exclusively on soil laboratory microcosm experiments (Table 3.2). The length of the reported microcosm studies ranged from 7 days to 96 months. In one study, no PAH biodegradation was observed over 500 days (Richardson et al., 2012), while in others greater than $50 \%$ degradation was observed in as little as 100 days (Espinoza \& Dendooven, 2007 and (Alvarez-Bernal et al., 2006). Half lives of 50 to 161 days were reported for mixtures of PAHs (Torlapati and Boufadel 2014). The rates shown in Table 3.2 do not show clear evidence that smaller PAHs biodegrade faster. The average first

order degradation rate constant for PAHs with 3 or less rings was $0.84 \mathrm{mg} / \mathrm{kg} / \mathrm{day}$, while PAHs with 4 or more rings have an average rate of $.92 \mathrm{mg} / \mathrm{kg} / \mathrm{day}$. 
Table 3.2: Reported Natural Attenuation Biodegradation Rates of PAHs in Soil

\begin{tabular}{|c|c|c|c|c|c|c|c|c|c|}
\hline \multirow[b]{2}{*}{ Compound } & \multirow[b]{2}{*}{$\begin{array}{l}\text { No. } \\
\text { Rings }\end{array}$} & \multirow[b]{2}{*}{ Matrix } & \multirow[b]{2}{*}{$\begin{array}{c}\text { Lab/ Field/ } \\
\text { Unknown } \\
\text { (L/F/U) }\end{array}$} & \multicolumn{2}{|c|}{$\begin{array}{c}\text { PAH Concentrations } \\
(\mathrm{mg} / \mathrm{kg})\end{array}$} & \multirow[b]{2}{*}{$\begin{array}{c}\text { Length of } \\
\text { study (days) }\end{array}$} & \multicolumn{2}{|c|}{ Biodegradation Kinetics } & \multirow[b]{2}{*}{ Reference } \\
\hline & & & & Initial & Final & & $\begin{array}{c}\text { Percent } \\
\text { Degraded }\end{array}$ & $\begin{array}{c}\text { Degradation } \\
\text { Rate (mg/kg/day) }\end{array}$ & \\
\hline 2-Ethenylnaphthalene & 2 & Soil & $\mathrm{L}$ & 50 & 43 & 150 & 14 & 0.047 & $\begin{array}{c}\text { Fava et al., } \\
2004\end{array}$ \\
\hline 2-Methylnaphthalene & 2 & Soil & $\mathrm{L}$ & 65 & 35.8 & 150 & 45 & 0.195 & $\begin{array}{l}\text { Fava et al., } \\
2004\end{array}$ \\
\hline Biphenyl & 2 & Soil & $\mathrm{L}$ & 1 & 0.7 & 21 & 30 & 0.015 & $\begin{array}{c}\text { Aronstein et } \\
\text { al, } 1991\end{array}$ \\
\hline Dimethylnaphthalene & 2 & Soil & $\mathrm{L}$ & 141 & 57.8 & 150 & 59 & 0.56 & $\begin{array}{c}\text { Fava et al., } \\
2004\end{array}$ \\
\hline Naphthalene & 2 & Soil & $\mathrm{L}$ & 42 & 19.7 & 150 & 53 & 0.15 & $\begin{array}{l}\text { Fava et al., } \\
2004\end{array}$ \\
\hline Naphthalene & 2 & Soil & $\mathrm{L}$ & 9.5 & 8.7 & 534 & 8 & 0.0015 & $\begin{array}{l}\text { Richardson } \\
\text { et al., } 2012^{1}\end{array}$ \\
\hline Naphthalene & 2 & Soil & $\mathrm{L}$ & 871 & 565 & 53 & 35 & 5.77 & $\begin{array}{c}\text { Tiehm et al., } \\
1997\end{array}$ \\
\hline Acenaphthene & 3 & Soil & $\mathrm{L}$ & 11.9 & 5.7 & 534 & 53 & 0.012 & $\begin{array}{l}\text { Richardson } \\
\text { et al., } 2012\end{array}$ \\
\hline Acenaphthene & 3 & Soil & $\mathrm{L}$ & 97 & 62.9 & 53 & 35 & 0.64 & $\begin{array}{c}\text { Tiehm et al., } \\
1997\end{array}$ \\
\hline Acenaphthene & 3 & Soil & $\mathrm{L}$ & 294 & 191 & 53 & 35 & 1.95 & $\begin{array}{c}\text { Tiehm et al., } \\
1997 \\
\end{array}$ \\
\hline Anthracene & 3 & Soil & $\mathrm{L}$ & 350 & 59.5 & 100 & 83 & 2.91 & $\begin{array}{c}\text { Alvarez- } \\
\text { Bernal et al., } \\
2006\end{array}$ \\
\hline
\end{tabular}




\begin{tabular}{|c|c|c|c|c|c|c|c|c|c|}
\hline \multirow[b]{2}{*}{ Compound } & \multirow[b]{2}{*}{$\begin{array}{l}\text { No. } \\
\text { Rings }\end{array}$} & \multirow[b]{2}{*}{ Matrix } & \multirow{2}{*}{$\begin{array}{c}\text { Lab/ Field/ } \\
\text { Unknown } \\
\text { (L/F/U) }\end{array}$} & \multicolumn{2}{|c|}{$\begin{array}{c}\text { PAH Concentrations } \\
(\mathrm{mg} / \mathrm{kg})\end{array}$} & \multirow[b]{2}{*}{$\begin{array}{l}\text { Length of } \\
\text { study (days) }\end{array}$} & \multicolumn{2}{|c|}{ Biodegradation Kinetics } & \multirow[b]{2}{*}{ Reference } \\
\hline & & & & Initial & Final & & $\begin{array}{c}\text { Percent } \\
\text { Degraded }\end{array}$ & $\begin{array}{c}\text { Degradation } \\
\text { Rate }(\mathrm{mg} / \mathrm{kg} / \text { day })\end{array}$ & \\
\hline Anthracene & 3 & Soil & $\mathrm{L}$ & 4002 & 3162 & 150 & 21 & 5.6 & $\begin{array}{c}\text { Fava et al., } \\
2004\end{array}$ \\
\hline Anthracene & 3 & Soil & $\mathrm{L}$ & 10.5 & 3.1 & 534 & 70 & 0.014 & $\begin{array}{l}\text { Richardson } \\
\text { et al., } 2012\end{array}$ \\
\hline Anthracene & 3 & Soil & $\mathrm{L}$ & 75 & 25 & 100 & 67 & 0.5 & $\begin{array}{c}\text { Rivera- } \\
\text { Espinoza \& } \\
\text { Dendooven, } \\
2007^{2}\end{array}$ \\
\hline Dibenzofuran & 3 & Soil & $\mathrm{L}$ & 355 & 198.8 & 150 & 44 & 1.04 & $\begin{array}{c}\text { Tiehm et al., } \\
1997 \\
\end{array}$ \\
\hline Fluorene & 3 & Soil & $\mathrm{L}$ & 970 & 776 & 150 & 20 & 1.29 & $\begin{array}{c}\text { Fava et al., } \\
2004\end{array}$ \\
\hline Fluorene & 3 & Soil & $\mathrm{L}$ & 83 & 30 & 60 & 64 & 0.88 & $\begin{array}{l}\text { Llado et al., } \\
2013\end{array}$ \\
\hline Fluorene & 3 & Soil & $\mathrm{L}$ & 9.5 & 3.6 & 534 & 62 & 0.011 & $\begin{array}{l}\text { Richardson } \\
\text { et al., } 2012\end{array}$ \\
\hline Fluorene & 3 & -- & -- & 308 & 200 & 53 & 35 & 2.04 & $\begin{array}{l}\text { Richardson } \\
\text { et al., } 2012\end{array}$ \\
\hline Phenanthrene & 3 & Soil & $\mathrm{L}$ & 500 & 15 & 100 & 97 & 4.85 & $\begin{array}{c}\text { Alvarez- } \\
\text { Bernal et al., } \\
2006\end{array}$ \\
\hline Phenanthrene & 3 & Soil & $\mathrm{L}$ & 1 & 0.952 & 21 & 5 & 0.0023 & $\begin{array}{l}\text { Aronstein et } \\
\text { al., } 1991\end{array}$ \\
\hline Phenanthrene & 3 & Slurry & $\mathrm{L}$ & 50 & 36.5 & 35 & 27 & 0.39 & $\begin{array}{c}\text { Providenti, } \\
\text { Flemming, } \\
\text { Lee, \& } \\
\text { Trevors, } \\
1995\end{array}$ \\
\hline
\end{tabular}




\begin{tabular}{|c|c|c|c|c|c|c|c|c|c|}
\hline \multirow[b]{2}{*}{ Compound } & \multirow[b]{2}{*}{$\begin{array}{l}\text { No. } \\
\text { Rings }\end{array}$} & \multirow[b]{2}{*}{ Matrix } & \multirow{2}{*}{$\begin{array}{c}\text { Lab/ Field/ } \\
\text { Unknown } \\
\text { (L/F/U) }\end{array}$} & \multicolumn{2}{|c|}{$\begin{array}{c}\text { PAH Concentrations } \\
(\mathrm{mg} / \mathrm{kg})\end{array}$} & \multirow[b]{2}{*}{$\begin{array}{c}\text { Length of } \\
\text { study (days) }\end{array}$} & \multicolumn{2}{|c|}{ Biodegradation Kinetics } & \multirow[b]{2}{*}{ Reference } \\
\hline & & & & Initial & Final & & $\begin{array}{l}\text { Percent } \\
\text { Degraded }\end{array}$ & $\begin{array}{c}\text { Degradation } \\
\text { Rate (mg/kg/day) }\end{array}$ & \\
\hline Phenanthrene & 3 & Slurry & $\mathrm{L}$ & 50 & 38.5 & 35 & 23 & 0.33 & $\begin{array}{c}\text { Providenti et } \\
\text { al., } 1995 \\
\end{array}$ \\
\hline Phenanthrene & 3 & Slurry & $\mathrm{L}$ & 114 & 31.5 & 27 & 72 & 3.06 & $\begin{array}{c}\text { Tiehm et al., } \\
1997^{3}\end{array}$ \\
\hline Phenanthrene & 3 & Soil & $\mathrm{L}$ & 129 & 37.2 & 534 & 71 & 0.17 & $\begin{array}{l}\text { Richardson } \\
\text { et al., } 2012\end{array}$ \\
\hline Phenanthrene & 3 & Soil & $\mathrm{L}$ & 325 & 163 & 100 & 50 & 1.62 & $\begin{array}{l}\text { Richardson } \\
\text { et al., } 2012\end{array}$ \\
\hline $\begin{array}{c}11 \mathrm{H}- \\
\text { Benzo[b]fluorene }\end{array}$ & 4 & Soil & $\mathrm{L}$ & 210 & 210 & 150 & 0 & 0 & $\begin{array}{c}\text { Tiehm et al., } \\
1997 \\
\end{array}$ \\
\hline Benz[a]anthracene & 4 & Soil & $\mathrm{L}$ & 13.8 & 7.1 & 534 & 49 & 0.0125 & $\begin{array}{l}\text { Richardson } \\
\text { et al., } 2012 \\
\end{array}$ \\
\hline Benz[a]anthracene & 4 & -- & -- & 254 & 165 & 53 & 35 & 1.68 & $\begin{array}{c}\text { Tiehm et al., } \\
1997 \\
\end{array}$ \\
\hline Benzo[a]anthracene & 4 & Soil & $\mathrm{L}$ & 37 & 21 & 62 & 43 & 0.26 & $\begin{array}{c}\text { Llado et al., } \\
2013\end{array}$ \\
\hline Chrysene & 4 & Soil & $\mathrm{L}$ & 68 & 40 & 63 & 41 & 0.44 & $\begin{array}{c}\text { Llado et al., } \\
2013 \\
\end{array}$ \\
\hline Chrysene & 4 & Soil & $\mathrm{L}$ & 14 & 6.7 & 534 & 52 & 0.137 & $\begin{array}{l}\text { Richardson } \\
\text { et al., } 2012 \\
\end{array}$ \\
\hline Chrysene & 4 & -- & -- & 173 & 112 & 53 & 35 & 1.15 & $\begin{array}{c}\text { Tiehm et al., } \\
1997\end{array}$ \\
\hline Fluoranthene & 4 & Soil & $\mathrm{L}$ & 2065 & 1652 & 150 & 20 & 2.75 & $\begin{array}{c}\text { Fava et al., } \\
2004\end{array}$ \\
\hline Fluoranthene & 4 & Soil & $\mathrm{L}$ & 25.2 & 11.3 & 534 & 55 & 0.026 & $\begin{array}{l}\text { Richardson } \\
\text { et al., } 2012\end{array}$ \\
\hline Fluoranthene & 4 & -- & -- & 681 & 442 & 53 & 35 & 4.51 & Tiehm et al., \\
\hline
\end{tabular}




\begin{tabular}{|c|c|c|c|c|c|c|c|c|c|}
\hline \multirow[b]{2}{*}{ Compound } & \multirow{2}{*}{$\begin{array}{l}\text { No. } \\
\text { Rings }\end{array}$} & \multirow[b]{2}{*}{ Matrix } & \multirow{2}{*}{$\begin{array}{c}\text { Lab/ Field/ } \\
\text { Unknown } \\
\text { (L/F/U) }\end{array}$} & \multicolumn{2}{|c|}{$\begin{array}{l}\text { PAH Concentrations } \\
(\mathbf{m g} / \mathbf{k g})\end{array}$} & \multirow{2}{*}{$\begin{array}{l}\text { Length of } \\
\text { study (days) }\end{array}$} & \multicolumn{2}{|c|}{ Biodegradation Kinetics } & \multirow[b]{2}{*}{ Reference } \\
\hline & & & & Initial & Final & & $\begin{array}{c}\text { Percent } \\
\text { Degraded }\end{array}$ & $\begin{array}{c}\text { Degradation } \\
\text { Rate }(\mathrm{mg} / \mathrm{kg} / \text { day })\end{array}$ & \\
\hline & & & & & & & & & 1997 \\
\hline Pyrene & 4 & Soil & $\mathrm{L}$ & 84 & 35 & 61 & 58 & 0.8 & $\begin{array}{c}\text { Llado et al., } \\
2013 \\
\end{array}$ \\
\hline Pyrene & 4 & Soil & $\mathrm{L}$ & 0.00688 & 0.00688 & 95 & 0 & 0 & $\begin{array}{c}\text { Cheung \& } \\
\text { Kinkle, } \\
2001\end{array}$ \\
\hline Pyrene & 4 & Soil & $\mathrm{L}$ & 0.00688 & 0.00647 & 95 & 6 & $4.26 \times 10^{-6}$ & $\begin{array}{c}\text { Cheung \& } \\
\text { Kinkle, } \\
2001\end{array}$ \\
\hline Pyrene & 4 & Soil & $\mathrm{L}$ & 0.00688 & 0.00673 & 95 & 2 & $1.49 \times 10^{-6}$ & $\begin{array}{c}\text { Cheung \& } \\
\text { Kinkle, } \\
2001\end{array}$ \\
\hline Pyrene & 4 & Soil & $\mathrm{L}$ & 0.00688 & 0.00659 & 95 & 4 & $2.98 \times 10^{-6}$ & $\begin{array}{c}\text { Cheung \& } \\
\text { Kinkle, } \\
2001\end{array}$ \\
\hline Pyrene & 4 & Soil & $\mathrm{L}$ & 1215 & 1215 & 150 & 0 & 0 & $\begin{array}{c}\text { Fava et al., } \\
2004 \\
\end{array}$ \\
\hline Pyrene & 4 & Soil & $\mathrm{L}$ & 100 & 35 & 32 & 65 & 2.03 & $\begin{array}{c}\text { Hwang \& } \\
\text { Cutright, } \\
2002 \\
\end{array}$ \\
\hline Pyrene & 4 & Soil & $\mathrm{L}$ & 100 & 21.7 & 32 & 78 & 2.45 & $\begin{array}{c}\text { Hwang \& } \\
\text { Cutright, } \\
2002\end{array}$ \\
\hline Pyrene & 4 & Soil & $\mathrm{L}$ & 100 & 18.2 & 32 & 82 & 2.56 & $\begin{array}{c}\text { Hwang \& } \\
\text { Cutright, } \\
2002\end{array}$ \\
\hline Pyrene & 4 & Soil & $\mathrm{L}$ & 40.9 & 18.4 & 534 & 55 & 0.042 & $\begin{array}{l}\text { Richardson } \\
\text { et al., } 2012\end{array}$ \\
\hline
\end{tabular}




\begin{tabular}{|c|c|c|c|c|c|c|c|c|c|}
\hline \multirow[b]{2}{*}{ Compound } & \multirow[b]{2}{*}{$\begin{array}{l}\text { No. } \\
\text { Rings }\end{array}$} & \multirow[b]{2}{*}{ Matrix } & \multirow[b]{2}{*}{$\begin{array}{c}\text { Lab/ Field/ } \\
\text { Unknown } \\
\text { (L/F/U) }\end{array}$} & \multicolumn{2}{|c|}{$\begin{array}{c}\text { PAH Concentrations } \\
(\mathrm{mg} / \mathrm{kg})\end{array}$} & \multirow[b]{2}{*}{$\begin{array}{l}\text { Length of } \\
\text { study (days) }\end{array}$} & \multicolumn{2}{|c|}{ Biodegradation Kinetics } & \multirow[b]{2}{*}{ Reference } \\
\hline & & & & Initial & Final & & $\begin{array}{c}\text { Percent } \\
\text { Degraded }\end{array}$ & $\begin{array}{c}\text { Degradation } \\
\text { Rate }(\mathrm{mg} / \mathrm{kg} / \text { day })\end{array}$ & \\
\hline Pyrene & 4 & Soil & $\mathrm{L}$ & 448 & 291 & 53 & 35 & 2.97 & $\begin{array}{c}\text { Tiehm et al., } \\
1997\end{array}$ \\
\hline Triphenylene & 4 & Soil & $\mathrm{L}$ & 302 & 302 & 150 & 0 & 0 & $\begin{array}{l}\text { Fava et al., } \\
2004\end{array}$ \\
\hline Benzo[a]pyrene & 5 & Soil & $\mathrm{L}$ & 22 & 17 & 66 & 23 & 0.76 & $\begin{array}{l}\text { Llado et al., } \\
2013\end{array}$ \\
\hline Benzo[a]pyrene & 5 & Soil & $\mathrm{L}$ & 150 & 39 & 7 & 74 & 0.16 & $\begin{array}{l}\text { Armenante, } \\
\text { et al } 1994\end{array}$ \\
\hline Benzo[a]pyrene & 5 & Soil & $\mathrm{L}$ & 13.5 & 10.4 & 534 & 23 & 0.0058 & $\begin{array}{l}\text { Richardson } \\
\text { et al., } 2012\end{array}$ \\
\hline Benzo[a]pyrene & 5 & Unknown & $\mathrm{U}$ & 169 & 110 & 53 & 35 & 1.12 & $\begin{array}{c}\text { Tiehm et al., } \\
1997\end{array}$ \\
\hline Benzo[a]pyrene & 5 & Soil & $\mathrm{L}$ & 95 & 66 & 100 & 31 & 0.29 & $\begin{array}{c}\text { Rivera- } \\
\text { Espinoza \& } \\
\text { Dendooven, } \\
2007 \\
\end{array}$ \\
\hline Benzo[k]fluoranthene & 5 & Soil & $\mathrm{L}$ & 38 & 24 & 65 & 37 & 0.215 & $\begin{array}{l}\text { Llado et al., } \\
2013\end{array}$ \\
\hline Benzo[k]fluoranthene & 5 & Soil & $\mathrm{L}$ & 4.2 & 3.2 & 534 & 24 & 0.0019 & $\begin{array}{l}\text { Richardson } \\
\text { et al., } 2012\end{array}$ \\
\hline Benzo[k]fluoranthene & 5 & Soil & $\mathrm{L}$ & 158 & 103 & 53 & 35 & 1.05 & $\begin{array}{c}\text { Tiehm et al., } \\
1997 \\
\end{array}$ \\
\hline Benzo[g,h,i]perylene & 6 & Soil & $\mathrm{L}$ & 5.3 & 5.3 & 534 & 0 & 0 & $\begin{array}{l}\text { Richardson } \\
\text { et al., } 2012\end{array}$ \\
\hline Acenaphthene & 3 & Soil & $\mathrm{L}$ & 11.9 & 5.7 & 534 & 52 & 0.0116 & $\begin{array}{c}\text { Cheung \& } \\
\text { Kinkle, } \\
2001\end{array}$ \\
\hline
\end{tabular}




\begin{tabular}{|c|c|c|c|c|c|c|c|c|c|}
\hline \multirow[b]{2}{*}{ Compound } & \multirow[b]{2}{*}{$\begin{array}{l}\text { No. } \\
\text { Rings }\end{array}$} & \multirow[b]{2}{*}{ Matrix } & \multirow[b]{2}{*}{$\begin{array}{c}\text { Lab/ Field/ } \\
\text { Unknown } \\
\text { (L/F/U) }\end{array}$} & \multicolumn{2}{|c|}{$\begin{array}{c}\text { PAH Concentrations } \\
(\mathrm{mg} / \mathrm{kg})\end{array}$} & \multirow[b]{2}{*}{$\begin{array}{l}\text { Length of } \\
\text { study (days) }\end{array}$} & \multicolumn{2}{|c|}{ Biodegradation Kinetics } & \multirow[b]{2}{*}{ Reference } \\
\hline & & & & Initial & Final & & $\begin{array}{l}\text { Percent } \\
\text { Degraded }\end{array}$ & $\begin{array}{c}\text { Degradation } \\
\text { Rate }(\mathrm{mg} / \mathrm{kg} / \text { day })\end{array}$ & \\
\hline Acenaphthene & 3 & Unknown & $\mathrm{U}$ & 97 & -- & 53 & -- & -- & $\begin{array}{c}\text { Cheung \& } \\
\text { Kinkle, } \\
2001\end{array}$ \\
\hline Acenaphthene & 3 & Unknown & $\mathrm{U}$ & 294 & -- & 53 & -- & -- & $\begin{array}{c}\text { Cheung \& } \\
\text { Kinkle, } \\
2001\end{array}$ \\
\hline Benzo[b]fluoranthene & 5 & Soil & $\mathrm{L}$ & 57 & 39 & 64 & 32 & 0.28 & $\begin{array}{l}\text { Llado et al., } \\
2013\end{array}$ \\
\hline Benzo[b]fluoranthene & 5 & Soil & $\mathrm{L}$ & 6.9 & 5.2 & 534 & 25 & 0.0032 & $\begin{array}{l}\text { Richardson } \\
\text { et al., } 2012\end{array}$ \\
\hline Benzo[b]fluoranthene & 5 & Soil & $\mathrm{L}$ & 125 & 81 & 53 & 35 & 0.83 & $\begin{array}{c}\text { Tiehm et al., } \\
1997 \\
\end{array}$ \\
\hline PAH mixture & varied & Soil & $\mathrm{L}$ & 293 & NSD & 1140 & NSD & -- & $\begin{array}{c}\text { Ouvrard et } \\
\text { al., } 2013\end{array}$ \\
\hline PAH mixture & varied & Soil & $\mathrm{L}$ & 1371 & NSD & 2490 & NSD & -- & $\begin{array}{c}\text { Ouvrard et } \\
\text { al., } 2013\end{array}$ \\
\hline PAH mixture & varied & Soil & $\mathrm{L}$ & 446 & NSD & 2880 & NSD & -- & $\begin{array}{l}\text { Ouvrard et } \\
\text { al., } 2013\end{array}$ \\
\hline PAH mixture & varied & Soil & $\mathrm{L}$ & 555 & 383 & 2880 & 31 & 0.06 & $\begin{array}{l}\text { Ouvrard et } \\
\text { al., } 2013\end{array}$ \\
\hline
\end{tabular}


Table 3.2 Notes

${ }^{1}$ Nitrogen-limited

${ }^{2}$ Nitrogen-sufficient

${ }^{3}$ Percent degradation is contributable to biodegradation only (dissolution and sampling contributed to percent PAH removed in this study). Final concentration calculated using only attenuation due to biodegradation.

${ }^{4}$ Percent degradation is total PAH degradation, not individual constituent.

${ }^{5}$ Percent degradation is total PAH degradation.

${ }^{6}$ Concentration read from graph. Total sum of initial anthracene, phenanthrene, and benzo[a]pyrene concentrations was provided (988 $\mathrm{mg} / \mathrm{kg}$ ); percent degradation of each individual PAH constituent was also provided.

${ }^{7}$ Concentration estimated from graph provided in article 


\subsection{PCB Biodegradation}

PCB biodegradation follows complex biodegradation pathways, which make it difficult to model their biodegradation rate. The rate is dependent on microbial population dynamics and soil conditions, both of which could change over time (Borja et al. 2005). However, laboratory experiments often overestimate biodegradation rates in the field because laboratory studies often use freshly spiked contaminants, which are more bioavailable than weathered contaminants, which are sequestered in the soil matrix (Rehmann and Daugulis 2008; WHO 1992). It is also difficult to predict rates because of the mixture of congeners in an environment, which may affect each others biodegradation. Because of this and other considerations, it is difficult to extrapolate field rates from laboratory experiments (WHO 1992). Natural attenuation rates are important to note because they can be used to predict what can be expected at SSFL. These rates are valuable because they take into account factors such as population dynamics and field conditions.

Some PCB natural attenuation rates are listed in Table 3.3. PCB contamination of river sediments has been the focus of most bioremediation studies because of large historical spills in aquatic environments, most notably the Hudson River (US EPA 2010). While rates of PCB natural attenuation for river sediments are shown in Table 3.3, it should be noted that microbial populations and metabolism in an aquatic environment are expected to differ greatly from those in soil. To provide an analysis of published data most pertinent to SSFL, the focus here is on studies done with soils rather than aquatic sediments. 
It is also important to know which organisms are known biodegraders so that they can be specifically looked for to assess the feasibility of natural attenuation. Because of the recalcitrant nature of PCBs, relatively few microorganisms have been isolated that can biodegrade them. The presence of any of these microbes on a site would indicate that natural attenuation of PCBs may be possible. Table 3.4 shows a list of 16 unique microbes in 254 experiments that are shown to biodegrade PCBs based on the literature. 
Table 3.3: Biodegradation Rates of PCBs under Natural Attenuation Conditions

\begin{tabular}{|c|c|c|c|c|c|c|c|c|}
\hline \multirow[b]{2}{*}{ Compound } & \multirow[b]{2}{*}{ Matrix } & \multirow[b]{2}{*}{$\begin{array}{l}\text { Lab/Field } \\
\quad(\mathbf{L} / \mathbf{F})\end{array}$} & \multicolumn{2}{|c|}{$\begin{array}{c}\text { Sediment PCB } \\
\text { Concentration }(\mathrm{nM}) \\
\end{array}$} & \multirow{2}{*}{$\begin{array}{c}\text { Length } \\
\text { of } \\
\text { Study } \\
\text { (days) }\end{array}$} & \multicolumn{2}{|c|}{ Biodegradation Kinetics } & \multirow[b]{2}{*}{ Reference } \\
\hline & & & Initial & Final & & $\begin{array}{l}\text { Percent } \\
\text { Degraded }\end{array}$ & $\begin{array}{c}\text { Degradation } \\
\text { Rate } \\
\text { (mg/kg/day) }\end{array}$ & \\
\hline $\begin{array}{c}2,3,4,2^{\prime}, 4^{\prime}, 5^{\prime}- \\
\text { hexaCBP }\end{array}$ & $\begin{array}{c}\text { river } \\
\text { sediment }\end{array}$ & $\mathrm{L}$ & 2250 & 608 & 450 & 73 & 4 & Rhee et al., 1993 \\
\hline $\begin{array}{c}2,3,4,5,6- \\
\text { penta CBP }\end{array}$ & $\begin{array}{c}\text { river } \\
\text { sediment }\end{array}$ & $\mathrm{L}$ & 1850 & 925 & 450 & 50 & 2 & Rhee et al., 1993 \\
\hline $3,4,3^{\prime} 4^{\prime} \mathrm{CBP}$ & $\begin{array}{c}\text { river } \\
\text { sediment }\end{array}$ & $\mathrm{L}$ & -- & -- & 450 & 0 & -- & Rhee et al., 1993 \\
\hline $\begin{array}{c}2,4,5,2^{\prime}, 4^{\prime}, 5^{\prime}- \\
\text { CBP }\end{array}$ & $\begin{array}{c}\text { river } \\
\text { sediment }\end{array}$ & $\mathrm{L}$ & 525 & 525 & 600 & 0 & 0 & Rhee et al., 1993 \\
\hline $\begin{array}{c}2,4,2^{\prime}, 4^{\prime} \\
\text { CBPs }\end{array}$ & $\begin{array}{c}\text { river } \\
\text { sediment }\end{array}$ & $\mathrm{L}$ & -- & -- & 450 & 0 & -- & Rhee et al., 1993 \\
\hline \multicolumn{3}{|c|}{--} & \multicolumn{2}{|c|}{$\begin{array}{c}\text { Soil PCB Concentration } \\
(\mathrm{mg} / \mathrm{kg})\end{array}$} & \multicolumn{4}{|c|}{--} \\
\hline PCB mixture & $\begin{array}{c}\text { sedime } \\
\mathrm{nt}\end{array}$ & $\mathrm{F}$ & 39.4 & 22.9 & 73 & 42 & 0.23 & $\begin{array}{c}\text { Harkness et al., } \\
1993 \\
\end{array}$ \\
\hline $\begin{array}{c}\text { Aroclor } \\
1260 \\
\end{array}$ & soil & $\mathrm{L}$ & 264.6 & -- & 200 & -- & $\begin{array}{c}0.008 \\
\text { mol\%/day } \\
\end{array}$ & $\begin{array}{c}\text { Kjellerup et al., } \\
2012 \\
\end{array}$ \\
\hline PCB mixture & soil & $\mathrm{L}$ & 2.1 & 1.9 & 415 & 10 & -- & Krumins et al., 2009 \\
\hline $\begin{array}{l}\text { Clophen } \\
\text { A30 } \\
\end{array}$ & soil & $\mathrm{L}$ & 52000 & 37960 & 42 & 27 & 334 & Viisimaa et al., 2013 \\
\hline
\end{tabular}


Table 3.3 Notes

${ }^{1}$ Initial and final concentrations estimated from graphs and calculated based on percent degradation, respectively, provided in article.

${ }^{2}$ Initial and final concentrations not provided in article.

${ }^{3}$ Each collected soil sample contained a varying amount of PCBs; the most representative sample was cited here.

${ }^{4}$ Actual percentage removal of PCBs for unamended soils was not provided in article 
Table 3.4: PCB-degrading bacterial species

Notes: NS: Not stated

\begin{tabular}{|c|c|c|c|c|c|}
\hline $\begin{array}{c}\text { PCB } \\
\text { congener } \\
\# \\
\end{array}$ & $\begin{array}{c}\text { Microorganism Involved in } \\
\text { Degradation }\end{array}$ & $\begin{array}{l}\text { Observed Rate of } \\
\text { Degradation }\end{array}$ & Metabolites & $\begin{array}{c}\begin{array}{c}\text { Number } \\
\text { of }\end{array} \\
\text { Chlorines } \\
\end{array}$ & Reference \\
\hline 1 & Burkholderia sp. LB400 & $100 \% / 24 \mathrm{~h}$ & NS & 1 & Rein et al. 2007 \\
\hline 1 & Rhodococcus sp. SK-1 & $40 \%$ / 4 days & NS & 1 & Chang et al. 2013 \\
\hline 1 & Rhodococcus sp. SK-3 & $45 \%$ / 4 days & NS & 1 & Chang et al. 2013 \\
\hline 1 & Rhodococcus sp. SK-4 & $35 \% / 4$ days & NS & 1 & Chang et al. 2013 \\
\hline 1 & Aquamicrobium defluvii SK-2 & $28 \%$ / 4 days & NS & 1 & Chang et al. 2013 \\
\hline 2 & Rhodococcus sp. SK-1 & $64.4 \%$ / 4 days & NS & 1 & Chang et al. 2013 \\
\hline 2 & Rhodococcus sp. SK-3 & $64.4 \%$ / 4 days & NS & 1 & Chang et al. 2013 \\
\hline 2 & Aquamicrobium defluvii SK-2 & $42 \% / 4$ days & NS & 1 & Chang et al. 2013 \\
\hline 3 & Burkholderia sp. LB400 & $100 \% / 22.7 \mathrm{~h}$ & NS & 1 & Rein et al. 2007 \\
\hline 3 & Rhodococcus sp. SK-1 & $66 \%$ / 4 days & NS & 1 & Chang et al. 2013 \\
\hline 3 & Rhodococcus sp. SK-3 & $60 \%$ / 4 days & NS & 1 & Chang et al. 2013 \\
\hline 3 & Rhodococcus sp. SK-4 & $50 \%$ / 4 days & NS & 1 & Chang et al. 2013 \\
\hline 3 & Aquamicrobium defluvii SK-2 & $31.4 \%$ / 4 days & NS & 1 & Chang et al. 2013 \\
\hline 4 & Corynebacterium sp. MB1 & $87 \% / 24 \mathrm{~h}$ at $5 \mathrm{uM}$ & NS & 2 & Bedard et al. 1986 \\
\hline 4 & Alcaligenes eutrophus H850 & $100 \% / 24 \mathrm{~h}$ at $5 \mathrm{uM}$ and $25 \mathrm{uM}$ & NS & 2 & Bedard et al. 1986 \\
\hline
\end{tabular}




\begin{tabular}{|c|c|c|c|c|c|}
\hline $\begin{array}{c}\text { PCB } \\
\text { congener } \\
\# \\
\end{array}$ & $\begin{array}{c}\text { Microorganism Involved in } \\
\text { Degradation }\end{array}$ & $\begin{array}{l}\text { Observed Rate of } \\
\text { Degradation }\end{array}$ & Metabolites & \begin{tabular}{|c|}
$\begin{array}{c}\text { Number } \\
\text { of } \\
\text { Chlorines }\end{array}$ \\
\end{tabular} & Reference \\
\hline 4 & Rhodococcus sp. SK-1 & $14.2 \%$ / 4 days & NS & 2 & Chang et al. 2013 \\
\hline 4 & Rhodococcus sp. SK-3 & $16 \% / 4$ days & NS & 2 & Chang et al. 2013 \\
\hline 4 & Rhodococcus sp. SK-4 & $22 \%$ / 4 days & NS & 2 & Chang et al. 2013 \\
\hline 4 & Aquamicrobium defluvii SK-2 & $34.7 \%$ / 4 days & NS & 2 & Chang et al. 2013 \\
\hline 5 & Corynebacterium sp. MB1 & $100 \% / 24 \mathrm{~h}$ at $5 \mathrm{uM}$ and $25 \mathrm{uM}$ & NS & 2 & Bedard et al. 1986 \\
\hline 5 & Alcaligenes eutrophus $\mathrm{H} 850$ & $100 \% / 24 \mathrm{~h}$ at $5 \mathrm{uM}$ and $25 \mathrm{uM}$ & NS & 2 & Bedard et al. 1986 \\
\hline 5 & Burkholderia sp. LB400 & $99 \% / 20.8 \mathrm{~h}$ & NS & 2 & Rein et al. 2007 \\
\hline 6 & Rhodococcus sp. SK-1 & $33.7 \%$ / 4 days & NS & 2 & Chang et al. 2013 \\
\hline 6 & Rhodococcus sp. SK-3 & $35 \% / 4$ days & NS & 2 & Chang et al. 2013 \\
\hline 6 & Rhodococcus sp. SK-4 & $34.8 \%$ / 4 days & NS & 2 & Chang et al. 2013 \\
\hline 6 & Aquamicrobium defluvii SK-2 & $34.8 \%$ / 4 days & NS & 2 & Chang et al. 2013 \\
\hline 6 & Sphingomonas sp. & $77 \% / 72 \mathrm{~h}$ & NS & 2 & Yong-lei et al. 2011 \\
\hline 6 & Pseudomonas sp. & $86 \% / 72 h$ & NS & 2 & Yong-lei et al. 2011 \\
\hline 8 & Corynebacterium sp. MB1 & $100 \% / 24 \mathrm{~h}$ at $5 \mathrm{uM}$ and $25 \mathrm{uM}$ & NS & 2 & Bedard et al. 1986 \\
\hline 8 & Alcaligenes eutrophus H850 & $100 \% / 24 \mathrm{~h}$ at $5 \mathrm{uM}$ and $25 \mathrm{uM}$ & NS & 2 & Bedard et al. 1986 \\
\hline 8 & Rhodococcus sp. SK-1 & $20 \%$ / 4 days & NS & 2 & Chang et al. 2013 \\
\hline 8 & Rhodococcus sp. SK-3 & $21 \%$ / 4 days & NS & 2 & Chang et al. 2013 \\
\hline 8 & Rhodococcus sp. SK-4 & $22 \% / 4$ days & NS & 2 & Chang et al. 2013 \\
\hline
\end{tabular}




\begin{tabular}{|c|c|c|c|c|c|}
\hline $\begin{array}{c}\text { PCB } \\
\text { congener } \\
\# \\
\end{array}$ & $\begin{array}{c}\text { Microorganism Involved in } \\
\text { Degradation }\end{array}$ & $\begin{array}{l}\text { Observed Rate of } \\
\text { Degradation }\end{array}$ & Metabolites & $\begin{array}{l}\text { Number } \\
\text { of } \\
\text { Chlorines }\end{array}$ & Reference \\
\hline 8 & Aquamicrobium defluvii SK-2 & $21.3 \%$ / 4 days & NS & 2 & Chang et al. 2013 \\
\hline 8 & Sphingomonas sp. & $70 \% / 72 h$ & NS & 2 & Yong-lei et al. 2011 \\
\hline 8 & Pseudomonas sp. & $87 \% / 72 h$ & NS & 2 & Yong-lei et al. 2011 \\
\hline 9 & Rhodococcus sp. SK-1 & $23.6 \%$ / 4 days & NS & 2 & Chang et al. 2013 \\
\hline 9 & Rhodococcus sp. SK-3 & $26 \% / 4$ days & NS & 2 & Chang et al. 2013 \\
\hline 9 & Rhodococcus sp. SK-4 & $21 \%$ / 4 days & NS & 2 & Chang et al. 2013 \\
\hline 9 & Aquamicrobium defluvii SK-2 & $19.1 \% / 4$ days & NS & 2 & Chang et al. 2013 \\
\hline 9 & Sphingomonas sp. & $51 \% / 72 \mathrm{~h}$ & NS & 2 & Yong-lei et al. 2011 \\
\hline 9 & Pseudomonas sp. & $99 \% / 72 h$ & NS & 2 & Yong-lei et al. 2011 \\
\hline 15 & Corynebacterium sp. MB1 & $100 \% / 24 \mathrm{~h}$ at $5 \mathrm{uM}$ and $25 \mathrm{uM}$ & NS & 3 & Bedard et al. 1986 \\
\hline 15 & Alcaligenes eutrophus $\mathrm{H} 850$ & $61 \% / 24 \mathrm{~h}$ at $5 \mathrm{uM}$ & NS & 3 & Bedard et al. 1986 \\
\hline 16 & Burkholderia sp. LB400 & $91 \% / 20.2 \mathrm{~h}$ & NS & 3 & Rein et al. 2007 \\
\hline 16 & Rhodococcus sp. SK-1 & $15.9 \% / 4$ days & NS & 3 & Chang et al. 2013 \\
\hline 16 & Rhodococcus sp. SK-3 & $17 \%$ / 4 days & NS & 3 & Chang et al. 2013 \\
\hline 16 & Rhodococcus sp. SK-4 & $17 \% / 4$ days & NS & 3 & Chang et al. 2013 \\
\hline 16 & Aquamicrobium defluvii SK-2 & $37 \% / 4$ days & NS & 3 & Chang et al. 2013 \\
\hline 17 & Burkholderia sp. LB400 & $97 \% / 22.2 \mathrm{~h}$ & NS & 3 & Rein et al. 2007 \\
\hline 17 & Ralstonia sp. SA-4 & $70.5 \% / 261 \mathrm{~h}$ & $\begin{array}{c}\text { CBA, } \\
\text { chloride }\end{array}$ & 3 & Adebusoye et al. 2008 \\
\hline
\end{tabular}




\begin{tabular}{|c|c|c|c|c|c|}
\hline $\begin{array}{c}\text { PCB } \\
\text { congener } \\
\# \\
\end{array}$ & $\begin{array}{c}\text { Microorganism Involved in } \\
\text { Degradation }\end{array}$ & $\begin{array}{l}\text { Observed Rate of } \\
\text { Degradation }\end{array}$ & Metabolites & $\begin{array}{c}\text { Number } \\
\text { of } \\
\text { Chlorines }\end{array}$ & Reference \\
\hline 17 & Pseudomonas sp. SA-6 & $86.2 \% / 261 \mathrm{~h}$ & $\begin{array}{c}\text { CBA, } \\
\text { chloride }\end{array}$ & 3 & Adebusoye et al. 2008 \\
\hline 17 & Sphingomonas sp. & $42 \% / 72 h$ & NS & 3 & Yong-lei et al. 2011 \\
\hline 17 & Pseudomonas sp. & $74 \% / 72 h$ & NS & 3 & Yong-lei et al. 2011 \\
\hline 18 & Corynebacterium sp. MB1 & $61 \% / 24 \mathrm{~h}$ at $5 \mathrm{uM}$ & NS & 3 & Bedard et al. 1986 \\
\hline 18 & Alcaligenes eutrophus $\mathrm{H} 850$ & $\begin{array}{c}100 \% / 24 \mathrm{~h} \text { at } 5 \mathrm{uM} \text { and } 98 \% / \\
24 \mathrm{~h} \text { at } 25 \mathrm{uM} \\
\end{array}$ & NS & 3 & Bedard et al. 1986 \\
\hline 18 & Burkholderia sp. LB400 & $92 \% / 19.8 \mathrm{~h}$ & NS & 3 & Rein et al. 2007 \\
\hline 18 & Paenibacillus sp. KBC101 & $100 \%$ / 3 days & NS & 3 & Sakai et al. 2004 \\
\hline 18 & Rhodococcus sp. RHA1 & $98 \%$ / 3 days & NS & 3 & Sakai et al. 2004 \\
\hline 18 & $\begin{array}{c}\text { Burkholderia xenovorans } \\
\text { LB400T } \\
\end{array}$ & $100 \% / 3$ days & NS & 3 & Sakai et al. 2004 \\
\hline 18 & $\begin{array}{c}\text { Pseudomonas } \\
\text { pseudoalcaligenes KF707 }\end{array}$ & $10 \% / 3$ days & NS & 3 & Sakai et al. 2004 \\
\hline 18 & Rhodococcus sp. SK-1 & $40 \%$ / 4 days & NS & 3 & Chang et al. 2013 \\
\hline 18 & Rhodococcus sp. SK-3 & $42 \%$ / 4 days & NS & 3 & Chang et al. 2013 \\
\hline 18 & Aquamicrobium defluvii SK-2 & $32 \% / 4$ days & NS & 3 & Chang et al. 2013 \\
\hline 18 & Ralstonia sp. SA-4 & $78.5 \% / 255 \mathrm{~h}$ & $\begin{array}{c}\text { CBA, } \\
\text { chloride }\end{array}$ & 3 & Adebusoye et al. 2008 \\
\hline 18 & Pseudomonas sp. SA-6 & $92.5 \% / 255 h$ & $\begin{array}{l}\text { CBA, } \\
\text { chloride }\end{array}$ & 3 & Adebusoye et al., 2008 \\
\hline 19 & Rhodococcus sp. SK-1 & $24.5 \%$ / 4 days & NS & 3 & Chang et al. 2013 \\
\hline 19 & Rhodococcus sp. SK-3 & $25 \% / 4$ days & NS & 3 & Chang et al. 2013 \\
\hline
\end{tabular}




\begin{tabular}{|c|c|c|c|c|c|}
\hline $\begin{array}{c}\text { PCB } \\
\text { congener } \\
\# \\
\end{array}$ & $\begin{array}{c}\text { Microorganism Involved in } \\
\text { Degradation }\end{array}$ & $\begin{array}{l}\text { Observed Rate of } \\
\text { Degradation }\end{array}$ & Metabolites & $\begin{array}{c}\text { Number } \\
\text { of } \\
\text { Chlorines }\end{array}$ & Reference \\
\hline 19 & Rhodococcus sp. SK-4 & $25 \% / 4$ days & NS & 3 & Chang et al., 2013 \\
\hline 19 & Aquamicrobium defluvii SK-2 & $18.5 \% / 4$ days & NS & 3 & Chang et al. 2013 \\
\hline 19 & Sphingomonas sp. & $49 \% / 72 h$ & NS & 3 & Yong-lei et al. 2011 \\
\hline 19 & Pseudomonas sp. & $82 \% / 72 h$ & NS & 3 & Yong-lei et al. 2011 \\
\hline 20 & Burkholderia sp. LB400 & $89 \%$ / 21.6h & NS & 3 & Rein et al. 2007 \\
\hline 22 & Rhodococcus sp. SK-1 & $23 \%$ / 4 days & NS & 3 & Chang et al. 2013 \\
\hline 22 & Rhodococcus sp. SK-3 & $24 \% / 4$ days & NS & 3 & Chang et al. 2013 \\
\hline 22 & Rhodococcus sp. SK-4 & $23 \%$ / 4 days & NS & 3 & Chang et al. 2013 \\
\hline 22 & Aquamicrobium defluvii SK-2 & $19.2 \%$ / 4 days & NS & 3 & Chang et al. 2013 \\
\hline 25 & Burkholderia sp. LB400 & $42 \% / 23.4 \mathrm{~h}$ & NS & 3 & Rein et al. 2007 \\
\hline 25 & Rhodococcus sp. SK-1 & $57 \%$ / 4 days & NS & 3 & Chang et al. 2013 \\
\hline 25 & Rhodococcus sp. SK-3 & $50 \% / 4$ days & NS & 3 & Chang et al. 2013 \\
\hline 25 & Rhodococcus sp. SK-4 & $50 \%$ / 4 days & NS & 3 & Chang et al. 2013 \\
\hline 25 & Aquamicrobium defluvii SK-2 & $37.8 \%$ / 4 days & NS & 3 & Chang et al. 2013 \\
\hline 27 & Sphingomonas sp. & $43 \% / 72 \mathrm{~h}$ & NS & 3 & Yong-lei et al. 2011 \\
\hline 27 & Pseudomonas sp. & $85 \% / 72 h$ & NS & 3 & Yong-lei et al. 2011 \\
\hline 28 & Stenotrophomonas sp. JSG1 & $50.3 \% / 48 h$ & NS & 3 & Ganesh-Kumar et al. 2013 \\
\hline 28 & Corynebacterium sp. MB1 & $\begin{array}{c}100 \% / 24 \mathrm{~h} \text { at } 5 \mathrm{uM} \text { and } 98 \% / \\
24 \mathrm{~h} \text { at } 25 \mathrm{uM}\end{array}$ & NS & 3 & (Bedard et al., 1986 \\
\hline
\end{tabular}




\begin{tabular}{|c|c|c|c|c|c|}
\hline $\begin{array}{c}\text { PCB } \\
\text { congener } \\
\# \\
\end{array}$ & $\begin{array}{c}\text { Microorganism Involved in } \\
\text { Degradation }\end{array}$ & $\begin{array}{l}\text { Observed Rate of } \\
\text { Degradation }\end{array}$ & Metabolites & $\begin{array}{l}\text { Number } \\
\text { of } \\
\text { Chlorines }\end{array}$ & Reference \\
\hline 28 & Alcaligenes eutrophus $\mathrm{H} 850$ & $76 \% / 24 \mathrm{~h}$ at $5 \mathrm{uM}$ & NS & 3 & Bedard et al. 1986 \\
\hline 28 & Rhodococcus sp. SK-1 & $22 \% / 4$ days & NS & 3 & Chang et al. 2013 \\
\hline 28 & Rhodococcus sp. SK-3 & $23 \%$ / 4 days & NS & 3 & Chang et al. 2013 \\
\hline 28 & Rhodococcus sp. SK-4 & $24 \%$ / 4 days & NS & 3 & Chang et al. 2013 \\
\hline 28 & Aquamicrobium defluvii SK-2 & $16.8 \% / 4$ days & NS & 3 & Chang et al. 2013 \\
\hline 31 & Stenotrophomonas sp. JSG1 & $60.4 \% / 48 \mathrm{~h}$ & NS & 3 & Ganesh-Kumar et al. 2012 \\
\hline 31 & Corynebacterium sp. MB1 & $\begin{array}{c}86 \% / 24 \mathrm{~h} \text { at } 5 \mathrm{uM} \text { and } 22 \% / \\
24 \mathrm{~h} \text { at } 25 \mathrm{uM}\end{array}$ & NS & 3 & Bedard et al. 1986 \\
\hline 31 & Alcaligenes eutrophus $\mathrm{H} 850$ & $\begin{array}{c}99 \% / 24 \mathrm{~h} \text { at } 5 \mathrm{uM} \text { and } 86 \% / \\
24 \mathrm{~h} \text { at } 25 \mathrm{uM}\end{array}$ & NS & 3 & Bedard et al. 1986 \\
\hline 31 & Burkholderia sp. LB400 & $92 \% / 20.8 \mathrm{~h}$ & NS & 3 & Rein et al. 2007 \\
\hline 34 & Burkholderia sp. LB400 & $80 \% / 22.2 \mathrm{~h}$ & NS & 3 & Rein et al. 2007 \\
\hline 40 & Corynebacterium sp. MB1 & $\begin{array}{c}100 \% / 24 \mathrm{~h} \text { at } 5 \mathrm{uM} \text { and } 96 \% \text { / } \\
24 \mathrm{~h} \text { at } 25 \mathrm{uM}\end{array}$ & NS & 3 & Bedard et al. 1986 \\
\hline 40 & Alcaligenes eutrophus $\mathrm{H} 850$ & $\begin{array}{c}99 \% / 24 \mathrm{~h} \text { at } 5 \mathrm{uM} \text { and } 53 \% / \\
24 \mathrm{~h} \text { at } 25 \mathrm{uM} \\
\end{array}$ & NS & 4 & Bedard et al. 1986 \\
\hline 40 & Pseudomonas sp. & $24 \% / 72 \mathrm{~h}$ & NS & 4 & Yong-lei et al. 2011 \\
\hline 41 & Burkholderia sp. LB400 & $39 \% / 22.5 \mathrm{~h}$ & NS & 4 & Rein et al. 2007 \\
\hline 43 & Stenotrophomonas sp. JSG1 & $100 \% / 48 h$ & NS & 4 & Ganesh-Kumar et al. 2012 \\
\hline 44 & Corynebacterium sp. MB1 & $\begin{array}{c}100 \% / 24 \mathrm{~h} \text { at } 5 \mathrm{uM} \text { and } 32 \% / \\
24 \mathrm{~h} \text { at } 25 \mathrm{uM}\end{array}$ & NS & 4 & Bedard et al. 1986 \\
\hline 44 & Alcaligenes eutrophus $\mathrm{H} 850$ & $\begin{array}{c}98 \% / 24 \mathrm{~h} \text { at } 5 \mathrm{uM} \text { and } 53 \% / \\
24 \mathrm{~h} \text { at } 25 \mathrm{uM} \\
\end{array}$ & NS & 4 & Bedard et al. 1986 \\
\hline 44 & Rhodococcus sp. SK-1 & $31 \%$ / 4 days & NS & 4 & Chang et al. 2013 \\
\hline
\end{tabular}




\begin{tabular}{|c|c|c|c|c|c|}
\hline $\begin{array}{c}\text { PCB } \\
\text { congener } \\
\# \\
\end{array}$ & $\begin{array}{c}\text { Microorganism Involved in } \\
\text { Degradation }\end{array}$ & $\begin{array}{l}\text { Observed Rate of } \\
\text { Degradation }\end{array}$ & Metabolites & $\begin{array}{c}\text { Number } \\
\text { of } \\
\text { Chlorines }\end{array}$ & Reference \\
\hline 44 & Rhodococcus sp. SK-3 & $30 \% / 4$ days & NS & 4 & Chang et al. 2013 \\
\hline 44 & Aquamicrobium defluvii SK-2 & $27 \%$ / 4 days & NS & 4 & Chang et al. 2013 \\
\hline 44 & Sphingomonas sp. & $17 \% / 72 \mathrm{~h}$ & NS & 4 & Yong-lei et al. 2011 \\
\hline 44 & Pseudomonas sp. & $29 \% / 72 h$ & NS & 4 & Yong-lei et al. 2011 \\
\hline 45 & Pseudomonas sp. & $44 \% / 72 \mathrm{~h}$ & NS & 4 & Yong-lei et al. 2011 \\
\hline 47 & Alcaligenes eutrophus $\mathrm{H} 850$ & $\begin{array}{c}54 \% / 24 \mathrm{~h} \text { at } 5 \mathrm{uM} \text { and } 5 \% / 24 \mathrm{~h} \\
\text { at } 25 \mathrm{uM} \\
\end{array}$ & NS & 4 & Bedard et al. 1986 \\
\hline 47 & Paenibacillus sp. KBC101 & $33 \%$ / 3 days & NS & 4 & Sakai et al. 2004 \\
\hline 47 & Rhodococcus sp. RHA1 & $83 \%$ / 3 days & NS & 4 & Sakai et al. 2004 \\
\hline 47 & $\begin{array}{l}\text { Burkholderia xenovorans } \\
\text { LB400T }\end{array}$ & $81 \% / 3$ days & NS & 4 & Sakai et al. 2004 \\
\hline 48 & Stenotrophomonas sp. JSG1 & $100 \% / 48 h$ & NS & 4 & Ganesh-Kumar et al. 2012 \\
\hline 49 & Sphingomonas sp. & $18 \% / 72 \mathrm{~h}$ & NS & 4 & Yong-lei et al. 2011 \\
\hline 49 & Pseudomonas sp. & $40 \% / 72 \mathrm{~h}$ & NS & 4 & Yong-lei et al. 2011 \\
\hline 52 & Stenotrophomonas sp. JSG1 & $100 \% / 48 h$ & NS & 4 & Ganesh-Kumar et al. 2012 \\
\hline 52 & Corynebacterium sp. MB1 & $17 \% / 24 \mathrm{~h}$ at $5 \mathrm{uM}$ & NS & 4 & Bedard et al. 1986 \\
\hline 52 & Alcaligenes eutrophus $\mathrm{H} 850$ & $\begin{array}{c}100 \% / 24 \mathrm{~h} \text { at } 5 \mathrm{uM} \text { and } 71 \% / \\
24 \mathrm{~h} \text { at } 25 \mathrm{uM} \\
\end{array}$ & NS & 4 & Bedard et al. 1986 \\
\hline 52 & Burkholderia sp. LB400 & $87 \% / 22.5 \mathrm{~h}$ & NS & 4 & Rein et al. 2007 \\
\hline 52 & Paenibacillus sp. KBC101 & $72 \%$ / 3 days & NS & 4 & Sakai et al. 2004 \\
\hline
\end{tabular}




\begin{tabular}{|c|c|c|c|c|c|}
\hline $\begin{array}{c}\text { PCB } \\
\text { congener } \\
\# \\
\end{array}$ & $\begin{array}{c}\text { Microorganism Involved in } \\
\text { Degradation }\end{array}$ & $\begin{array}{l}\text { Observed Rate of } \\
\text { Degradation }\end{array}$ & Metabolites & \begin{tabular}{|c|}
$\begin{array}{c}\text { Number } \\
\text { of } \\
\text { Chlorines }\end{array}$ \\
\end{tabular} & Reference \\
\hline 52 & Rhodococcus sp. RHA1 & $76 \% / 3$ days & NS & 4 & Sakai et al. 2004 \\
\hline 52 & $\begin{array}{l}\text { Burkholderia xenovorans } \\
\text { LB400T }\end{array}$ & $100 \%$ / 3 days & NS & 4 & Sakai et al. 2004 \\
\hline 52 & $\begin{array}{c}\text { Pseudomonas } \\
\text { pseudoalcaligenes KF707 }\end{array}$ & $9 \% / 3$ days & NS & 4 & Sakai et al. 2004 \\
\hline 52 & Rhodococcus sp. SK-1 & $29 \% / 4$ days & NS & 4 & Chang et al. 2013 \\
\hline 52 & Rhodococcus sp. SK-3 & $27 \% / 4$ days & NS & 4 & Chang et al. 2013 \\
\hline 52 & Rhodococcus sp. SK-4 & $28 \%$ / 4 days & NS & 4 & Chang et al. 2013 \\
\hline 52 & Aquamicrobium defluvii SK-2 & $37 \%$ / 4 days & NS & 4 & Chang et al. 2013 \\
\hline 52 & Sphingomonas sp. & $17 \% / 72 \mathrm{~h}$ & NS & 4 & Yong-lei et al. 2011 \\
\hline 52 & Pseudomonas sp. & $41 \% / 72 \mathrm{~h}$ & NS & 4 & Yong-lei et al. 2011 \\
\hline 53 & Stenotrophomonas sp. JSG1 & $100 \% / 48 \mathrm{~h}$ & NS & 4 & Ganesh-Kumar et al. 2012 \\
\hline 56 & Rhodococcus sp. SK-1 & $48 \%$ / 4 days & NS & 4 & Chang et al. 2013 \\
\hline 56 & Rhodococcus sp. SK-3 & $45 \%$ / 4 days & NS & 4 & Chang et al. 2013 \\
\hline 56 & Rhodococcus sp. SK-4 & $41 \%$ / 4 days & NS & 4 & Chang et al. 2013 \\
\hline 56 & Aquamicrobium defluvii SK-2 & $25 \% / 4$ days & NS & 4 & Chang et al. 2013 \\
\hline 66 & Corynebacterium sp. MB1 & $\begin{array}{c}93 \% / 24 \mathrm{~h} \text { at } 5 \mathrm{uM} \text { and } 64 \% / \\
24 \mathrm{~h} \text { at } 25 \mathrm{uM} \\
\end{array}$ & NS & 4 & Bedard et al. 1986 \\
\hline 66 & Alcaligenes eutrophus H850 & $22 \% / 24 \mathrm{~h}$ at $5 \mathrm{uM}$ & NS & 4 & Bedard et al. 1986 \\
\hline 66 & Paenibacillus sp. КBC101 & $58 \% / 3$ days & NS & 4 & Sakai et al. 2004 \\
\hline
\end{tabular}




\begin{tabular}{|c|c|c|c|c|c|}
\hline $\begin{array}{c}\text { PCB } \\
\text { congener } \\
\# \\
\end{array}$ & $\begin{array}{c}\text { Microorganism Involved in } \\
\text { Degradation }\end{array}$ & $\begin{array}{l}\text { Observed Rate of } \\
\text { Degradation }\end{array}$ & Metabolites & \begin{tabular}{|c|}
$\begin{array}{c}\text { Number } \\
\text { of } \\
\text { Chlorines }\end{array}$ \\
\end{tabular} & Reference \\
\hline 66 & Rhodococcus sp. RHA1 & $99 \% / 3$ days & NS & 4 & Sakai et al. 2004 \\
\hline 66 & $\begin{array}{c}\text { Burkholderia xenovorans } \\
\text { LB400T }\end{array}$ & $43 \%$ / 3 days & NS & 4 & Sakai et al. 2004 \\
\hline 66 & $\begin{array}{c}\text { Pseudomonas } \\
\text { pseudoalcaligenes } \mathrm{KF} 707\end{array}$ & $31 \% / 3$ days & NS & 4 & Sakai et al. 2004 \\
\hline 66 & Rhodococcus sp. SK-1 & $36 \% / 4$ days & NS & 4 & Chang et al. 2013 \\
\hline 66 & Rhodococcus sp. SK-3 & $37 \% / 4$ days & NS & 4 & Chang et al. 2013 \\
\hline 66 & Rhodococcus sp. SK-4 & $33 \% / 4$ days & NS & 4 & Chang et al. 2013 \\
\hline 66 & Aquamicrobium defluvii SK-2 & $17 \% / 4$ days & NS & 4 & Chang et al. 2013 \\
\hline 67 & Corynebacterium sp. MB1 & $\begin{array}{c}56 \% / 24 \mathrm{~h} \text { at } 5 \mathrm{uM} \text { and } 5 \% / 24 \mathrm{~h} \\
\text { at } 25 \mathrm{uM}\end{array}$ & NS & 4 & Bedard et al. 1986 \\
\hline 67 & Alcaligenes eutrophus H850 & $\begin{array}{c}90 \% / 24 \mathrm{~h} \text { at } 5 \mathrm{uM} \text { and } 54 \% / \\
24 \mathrm{~h} \text { at } 25 \mathrm{uM}\end{array}$ & NS & 4 & Bedard et al. 1986 \\
\hline 67 & Rhodococcus sp. SK-1 & $34.5 \% / 4$ days & NS & 4 & Chang et al. 2013 \\
\hline 67 & Rhodococcus sp. SK-3 & $32 \% / 4$ days & NS & 4 & Chang et al. 2013 \\
\hline 67 & Rhodococcus sp. SK-4 & $36 \% / 4$ days & NS & 4 & Chang et al. 2013 \\
\hline 67 & Aquamicrobium defluvii SK-2 & $48 \% / 4$ days & NS & 4 & Chang et al. 2013 \\
\hline 69 & Burkholderia sp. LB400 & $26 \% / 23 h$ & NS & 4 & Rein et al. 2007 \\
\hline 69 & Sphingomonas sp. & $16 \% / 72 \mathrm{~h}$ & NS & 4 & Yong-lei et al. 2011 \\
\hline 69 & Pseudomonas sp. & $30 \% / 72 \mathrm{~h}$ & NS & 4 & Yong-lei et al. 2011 \\
\hline 70 & Stenotrophomonas sp. JSG1 & $61.2 \% / 48 \mathrm{~h}$ & NS & 4 & $\begin{array}{c}\text { Ganesh-Kumar et al. } \\
42012\end{array}$ \\
\hline
\end{tabular}




\begin{tabular}{|c|c|c|c|c|c|}
\hline $\begin{array}{c}\text { PCB } \\
\text { congener } \\
\# \\
\end{array}$ & $\begin{array}{c}\text { Microorganism Involved in } \\
\text { Degradation }\end{array}$ & $\begin{array}{l}\text { Observed Rate of } \\
\text { Degradation }\end{array}$ & Metabolites & $\begin{array}{l}\text { Number } \\
\text { of } \\
\text { Chlorines }\end{array}$ & Reference \\
\hline 70 & Burkholderia sp. LB400 & $57 \% / 22.1 \mathrm{~h}$ & NS & 4 & Rein et al. 2007 \\
\hline 70 & Sphingomonas sp. & $13 \% / 72 \mathrm{~h}$ & NS & 4 & Yong-lei et al. 2011 \\
\hline 70 & Pseudomonas sp. & $20 \% / 72 h$ & NS & 4 & Yong-lei et al. 2011 \\
\hline 71 & Rhodococcus sp. SK-1 & $57 \%$ / 4 days & NS & 4 & Chang et al. 2013 \\
\hline 71 & Rhodococcus sp. SK-3 & $51 \% / 4$ days & NS & 4 & Chang et al. 2013 \\
\hline 71 & Rhodococcus sp. SK-4 & $54 \%$ / 4 days & NS & 4 & Chang et al. 2013 \\
\hline 71 & Aquamicrobium defluvii SK-2 & $56 \%$ / 4 days & NS & 4 & Chang et al. 2013 \\
\hline 74 & Stenotrophomonas sp. JSG1 & $100 \% / 48 h$ & NS & 4 & Ganesh-Kumar et al. 2012 \\
\hline 74 & Rhodococcus sp. SK-1 & $45.2 \%$ / 4 days & NS & 4 & Chang et al. 2013 \\
\hline 74 & Rhodococcus sp. SK-3 & $41 \%$ / 4 days & NS & 4 & Chang et al. 2013 \\
\hline 74 & Rhodococcus sp. SK-4 & $40 \%$ / 4 days & NS & 4 & Chang et al. 2013 \\
\hline 74 & Aquamicrobium defluvii SK-2 & $20.3 \%$ / 4 days & NS & 4 & Chang et al. 2013 \\
\hline 74 & Pseudomonas sp. & $26 \% / 72 \mathrm{~h}$ & NS & 4 & Yong-lei et al. 2011 \\
\hline 75 & Sphingomonas sp. & $14 \% / 72 \mathrm{~h}$ & NS & 4 & Yong-lei et al. 2011 \\
\hline 75 & Pseudomonas sp. & $38 \% / 72 \mathrm{~h}$ & NS & 4 & Yong-lei et al. 2011 \\
\hline 77 & Corynebacterium sp. MB1 & $21 \% / 24 \mathrm{~h}$ at $5 \mathrm{uM}$ & NS & 4 & Bedard et al. 1986 \\
\hline 77 & Paenibacillus sp. KBC101 & $56 \% / 3$ days & NS & 4 & Sakai et al. 2004 \\
\hline 77 & $\begin{array}{c}\text { Burkholderia xenovorans } \\
\text { LB400T }\end{array}$ & $6 \%$ / 3 days & NS & 4 & Sakai et al. 2004 \\
\hline
\end{tabular}




\begin{tabular}{|c|c|c|c|c|c|}
\hline $\begin{array}{c}\text { PCB } \\
\text { congener } \\
\# \\
\end{array}$ & $\begin{array}{c}\text { Microorganism Involved in } \\
\text { Degradation }\end{array}$ & $\begin{array}{l}\text { Observed Rate of } \\
\text { Degradation }\end{array}$ & Metabolites & \begin{tabular}{|c|}
$\begin{array}{c}\text { Number } \\
\text { of } \\
\text { Chlorines }\end{array}$ \\
\end{tabular} & Reference \\
\hline 82 & Stenotrophomonas sp. JSG1 & $35.6 \% / 48 \mathrm{~h}$ & NS & 5 & Ganesh-Kumar et al. 2012 \\
\hline 82 & Aquamicrobium defluvii SK-2 & $17 \% / 4$ days & NS & 5 & Chang et al. 2013 \\
\hline 85 & Stenotrophomonas sp. JSG1 & $21.4 \% / 48 \mathrm{~h}$ & NS & 5 & Ganesh-Kumar et al. 2012 \\
\hline 86 & Corynebacterium sp. MB1 & $\begin{array}{c}98 \% / 24 \mathrm{~h} \text { at } 5 \mathrm{uM} \text { and } 58 \% / \\
24 \mathrm{~h} \text { at } 25 \mathrm{uM} \\
\end{array}$ & NS & 5 & Bedard et al. 1986 \\
\hline 86 & Alcaligenes eutrophus H850 & $46 \% / 24 \mathrm{~h}$ at $5 \mathrm{uM}$ & NS & 5 & Bedard et al. 1986 \\
\hline 87 & Alcaligenes eutrophus H850 & $59 \% / 24 \mathrm{~h}$ at $5 \mathrm{uM}$ & NS & 5 & Bedard et al. 1986 \\
\hline 87 & Aquamicrobium defluvii SK-2 & $15 \% / 4$ days & NS & 5 & Chang et al. 2013 \\
\hline 91 & Stenotrophomonas sp. JSG1 & $23.7 \% / 48 \mathrm{~h}$ & NS & 5 & Ganesh-Kumar et al. 2012 \\
\hline 92 & Stenotrophomonas sp. JSG1 & $2.3 \% / 48 \mathrm{~h}$ & NS & 5 & Ganesh-Kumar et al. 2012 \\
\hline 99 & Stenotrophomonas sp. JSG1 & $2.5 \% / 48 \mathrm{~h}$ & NS & 5 & Ganesh-Kumar et al. 2012 \\
\hline 99 & Rhodococcus sp. SK-1 & $22 \% / 4$ days & NS & 5 & Chang et al. 2013 \\
\hline 99 & Rhodococcus sp. SK-3 & $20 \% / 4$ days & NS & 5 & Chang et al. 2013 \\
\hline 99 & Rhodococcus sp. SK-4 & $23 \% / 4$ days & NS & 5 & Chang et al. 2013 \\
\hline 99 & Aquamicrobium defluvii SK-2 & $11.7 \%$ / 4 days & NS & 5 & Chang et al. 2013 \\
\hline 101 & Corynebacterium sp. MB1 & $9 \% / 24 \mathrm{~h}$ at $5 \mathrm{uM}$ & NS & 5 & Bedard et al. 1986 \\
\hline 101 & Alcaligenes eutrophus H850 & $\begin{array}{c}70 \% / 24 \mathrm{~h} \text { at } 5 \mathrm{uM} \text { and } 30 \% / \\
24 \mathrm{~h} \text { at } 25 \mathrm{uM} \\
\end{array}$ & NS & 5 & Bedard et al. 1986 \\
\hline 101 & Burkholderia sp. LB400 & $47 \% / 21.9 \mathrm{~h}$ & NS & 5 & Rein et al. 2007 \\
\hline 101 & Paenibacillus sp. KBC101 & $58 \% / 3$ days & NS & 5 & Sakai et al. 2004 \\
\hline
\end{tabular}




\begin{tabular}{|c|c|c|c|c|c|}
\hline $\begin{array}{c}\text { PCB } \\
\text { congener } \\
\# \\
\end{array}$ & $\begin{array}{c}\text { Microorganism Involved in } \\
\text { Degradation }\end{array}$ & $\begin{array}{l}\text { Observed Rate of } \\
\text { Degradation }\end{array}$ & Metabolites & $\begin{array}{l}\text { Number } \\
\text { of } \\
\text { Chlorines }\end{array}$ & Reference \\
\hline 101 & Rhodococcus sp. RHA1 & $29 \%$ / 3 days & NS & 5 & Sakai et al. 2004 \\
\hline 101 & $\begin{array}{c}\text { Burkholderia xenovorans } \\
\text { LB400T }\end{array}$ & $100 \%$ / 3 days & NS & 5 & Sakai et al. 2004 \\
\hline 110 & Rhodococcus sp. SK-1 & $25 \% / 4$ days & NS & 5 & Chang et al. 2013 \\
\hline 110 & Rhodococcus sp. SK-3 & $23 \% / 4$ days & NS & 5 & Chang et al. 2013 \\
\hline 110 & Rhodococcus sp. SK-4 & $25 \% / 4$ days & NS & 5 & Chang et al. 2013 \\
\hline 110 & Aquamicrobium defluvii SK-2 & $12 \% / 4$ days & NS & 5 & Chang et al. 2013 \\
\hline 129 & Stenotrophomonas sp. JSG1 & $1.9 \% / 48 \mathrm{~h}$ & NS & 6 & Ganesh-Kumar et al. 2012 \\
\hline 138 & Rhodococcus sp. SK-1 & $21.2 \%$ / 4 days & NS & 6 & Chang et al. 2013 \\
\hline 138 & Rhodococcus sp. SK-3 & $22 \%$ / 4 days & NS & 6 & Chang et al. 2013 \\
\hline 138 & Rhodococcus sp. SK-4 & $21 \% / 4$ days & NS & 6 & Chang et al. 2013 \\
\hline 138 & Aquamicrobium defluvii SK-2 & $34 \%$ / 4 days & NS & 6 & Chang et al. 2013 \\
\hline 141 & Stenotrophomonas sp. JSG1 & $18.4 \% / 48 h$ & NS & 6 & Ganesh-Kumar et al. 2012 \\
\hline 141 & Stenotrophomonas sp. JSG1 & $2.0 \% / 48 h$ & NS & 6 & Ganesh-Kumar et al. 2012 \\
\hline 146 & Aquamicrobium defluvii SK-2 & $21.9 \%$ / 4 days & NS & 6 & Chang et al. 2013 \\
\hline 147 & Rhodococcus sp. SK-1 & $22 \% / 4$ days & NS & 6 & Chang et al. 2013 \\
\hline 147 & Rhodococcus sp. SK-3 & $24 \% / 4$ days & NS & 6 & Chang et al. 2013 \\
\hline 147 & Rhodococcus sp. SK-4 & $22 \% / 4$ days & NS & 6 & Chang et al. 2013 \\
\hline
\end{tabular}




\begin{tabular}{|c|c|c|c|c|c|}
\hline $\begin{array}{c}\text { PCB } \\
\text { congener } \\
\# \\
\end{array}$ & $\begin{array}{c}\text { Microorganism Involved in } \\
\text { Degradation }\end{array}$ & $\begin{array}{l}\text { Observed Rate of } \\
\text { Degradation }\end{array}$ & Metabolites & $\begin{array}{l}\text { Number } \\
\text { of } \\
\text { Chlorines }\end{array}$ & Reference \\
\hline 147 & Aquamicrobium defluvii SK-2 & $18.7 \%$ / 4 days & NS & 6 & Chang et al. 2013 \\
\hline 151 & Stenotrophomonas sp. JSG1 & $12.3 \% / 48 \mathrm{~h}$ & NS & 6 & Ganesh-Kumar et al. 2012 \\
\hline 153 & Stenotrophomonas sp. JSG1 & $3.5 \% / 48 h$ & NS & 6 & Ganesh-Kumar et al. 2012 \\
\hline 153 & Alcaligenes eutrophus $\mathrm{H} 850$ & $16 \% / 24 \mathrm{~h}$ at $5 \mathrm{uM}$ & NS & 6 & Bedard et al. 1986 \\
\hline 153 & Paenibacillus sp. KBC101 & $11 \% / 3$ days & NS & 6 & Sakai et al. 2004 \\
\hline 153 & $\begin{array}{c}\text { Burkholderia xenovorans } \\
\text { LB400T }\end{array}$ & $41 \%$ / 3 days & NS & 6 & Sakai et al. 2004 \\
\hline 153 & Rhodococcus sp. SK-1 & $18.5 \%$ / 4 days & NS & 6 & Chang et al. 2013 \\
\hline 153 & Rhodococcus sp. SK-3 & $19 \%$ / 4 days & NS & 6 & Chang et al. 2013 \\
\hline 153 & Rhodococcus sp. SK-4 & $19 \%$ / 4 days & NS & 6 & Chang et al. 2013 \\
\hline 153 & Aquamicrobium defluvii SK-2 & $31.8 \%$ / 4 days & NS & 6 & Chang et al. 2013 \\
\hline 173 & Rhodococcus sp. SK-1 & $16 \% / 4$ days & NS & 7 & Chang et al. 2013 \\
\hline 173 & Rhodococcus sp. SK-3 & $18 \%$ / 4 days & NS & 7 & Chang et al. 2013 \\
\hline 179 & Rhodococcus sp. SK-1 & $30.5 \%$ / 4 days & NS & 7 & Chang et al. 2013 \\
\hline 179 & Rhodococcus sp. SK-3 & $32 \%$ / 4 days & NS & 7 & Chang et al. 2013 \\
\hline 179 & Rhodococcus sp. SK-4 & $29 \% / 4$ days & NS & 7 & Chang et al. 2013 \\
\hline 179 & Aquamicrobium defluvii SK-2 & $17 \% / 4$ days & NS & 7 & Chang et al. 2013 \\
\hline 187 & Rhodococcus sp. SK-1 & $27 \%$ / 4 days & NS & 7 & Chang et al. 2013 \\
\hline
\end{tabular}




\begin{tabular}{|c|c|c|c|c|c|}
\hline $\begin{array}{c}\text { PCB } \\
\text { congener } \\
\# \\
\end{array}$ & $\begin{array}{c}\text { Microorganism Involved in } \\
\text { Degradation }\end{array}$ & $\begin{array}{l}\text { Observed Rate of } \\
\text { Degradation }\end{array}$ & Metabolites & $\begin{array}{c}\begin{array}{c}\text { Number } \\
\text { of } \\
\text { Chlorines }\end{array} \\
\end{array}$ & Reference \\
\hline 187 & Rhodococcus sp. SK-3 & $26 \%$ / 4 days & NS & 7 & Chang et al. 2013 \\
\hline 187 & Rhodococcus sp. SK-4 & $25 \%$ / 4 days & NS & 7 & Chang et al. 2013 \\
\hline 187 & Aquamicrobium defluvii SK-2 & $17 \%$ / 4 days & NS & 7 & Chang et al. 2013 \\
\hline 108,142 & Stenotrophomonas sp. JSG1 & $10.3 \% / 48 h$ & NS & 5,6 & Ganesh-Kumar et al. 2012 \\
\hline 118,134 & Stenotrophomonas sp. JSG1 & $27.8 \% / 48 h$ & NS & 5,6 & Ganesh-Kumar et al. 2012 \\
\hline 120,148 & Stenotrophomonas sp. JSG1 & $5.8 \% / 48 h$ & NS & 5,6 & Ganesh-Kumar et al. 2012 \\
\hline 123,142 & Stenotrophomonas sp. JSG1 & $1.5 \% / 48 \mathrm{~h}$ & NS & 5,6 & Ganesh-Kumar et al. 2012 \\
\hline 138,160 & Stenotrophomonas sp. JSG1 & $2.8 \% / 48 h$ & NS & 6,6 & Ganesh-Kumar et al. 2012 \\
\hline 15,18 & Sphingomonas sp. & $44 \% / 72 \mathrm{~h}$ & NS & 2,3 & Yong-lei et al. 2011 \\
\hline 15,18 & Pseudomonas sp. & $74 \% / 72 \mathrm{~h}$ & NS & 2,3 & Yong-lei et al. 2011 \\
\hline 16,32 & Sphingomonas sp. & $32 \% / 72 h$ & NS & 2,3 & Yong-lei et al. 2011 \\
\hline 16,32 & Pseudomonas sp. & $64 \% / 72 h$ & NS & 3,4 & Yong-lei et al. 2011 \\
\hline 22,51 & Sphingomonas sp. & $28 \% / 72 \mathrm{~h}$ & NS & 3,4 & Yong-lei et al. 2011 \\
\hline 22,51 & Pseudomonas sp. & $51 \% / 72 \mathrm{~h}$ & NS & 3,4 & Yong-lei et al. 2011 \\
\hline 25,26 & Sphingomonas sp. & $29 \% / 72 h$ & NS & 3,3 & Yong-lei et al. 2011 \\
\hline 25,26 & Pseudomonas sp. & $63 \% / 72 h$ & NS & 3,3 & Yong-lei et al. 2011 \\
\hline 28,31 & Sphingomonas sp. & $59 \% / 72 \mathrm{~h}$ & NS & 3,3 & Yong-lei et al. 2011 \\
\hline
\end{tabular}




\begin{tabular}{|c|c|c|c|c|c|}
\hline $\begin{array}{c}\text { PCB } \\
\text { congener } \\
\# \\
\end{array}$ & $\begin{array}{c}\text { Microorganism Involved in } \\
\text { Degradation }\end{array}$ & $\begin{array}{l}\text { Observed Rate of } \\
\text { Degradation }\end{array}$ & Metabolites & $\begin{array}{c}\text { Number } \\
\text { of } \\
\text { Chlorines }\end{array}$ & Reference \\
\hline 28,31 & Pseudomonas sp. & $64 \% / 72 h$ & NS & 3,3 & Yong-lei et al. 2011 \\
\hline 33,53 & Sphingomonas sp. & $47 \% / 72 \mathrm{~h}$ & NS & 3,3 & Yong-lei et al. 2011 \\
\hline 33,53 & Pseudomonas sp. & $43 \% / 72 h$ & NS & 3,4 & Yong-lei et al. 2011 \\
\hline 37,42 & Sphingomonas sp. & $19 \% / 72 h$ & NS & 3,4 & Yong-lei et al. 2011 \\
\hline 37,42 & Pseudomonas sp. & $28 \% / 72 \mathrm{~h}$ & NS & 3,4 & Yong-lei et al. 2011 \\
\hline 4 and 10 & Sphingomonas sp. & $64 \% / 72 h$ & NS & 2,2 & Yong-lei et al. 2011 \\
\hline 4 and 10 & Pseudomonas sp. & $90 \% / 72 \mathrm{~h}$ & NS & 2,2 & Yong-lei et al. 2011 \\
\hline 42,64 & Stenotrophomonas sp. JSG1 & $50.2 \% / 48 h$ & NS & 3,4 & Ganesh-Kumar et al. 2012 \\
\hline 56,60 & Stenotrophomonas sp. JSG1 & $40.1 \% / 48 h$ & NS & 4,4 & Ganesh-Kumar et al. 2012 \\
\hline 60,93 & Stenotrophomonas sp. JSG1 & $1.0 \% / 48 \mathrm{~h}$ & NS & 4,5 & Ganesh-Kumar et al. 2012 \\
\hline 77,109 & Stenotrophomonas sp. JSG1 & $1.1 \% / 48 \mathrm{~h}$ & NS & 4,5 & Ganesh-Kumar et al. 2012 \\
\hline 87,101 & Stenotrophomonas sp. JSG1 & $55.9 \% / 48 h$ & NS & 4,5 & Ganesh-Kumar et al. 2012 \\
\hline
\end{tabular}




\subsection{Dioxin Biodegradation}

As with PCBs, it is important to look at studies that measure natural attenuation rates as well as isolated biodegraders of dioxins. The field and laboratory experiments that provide natural attenuation rates are useful in predicting what may happen with SSFL. The identities of known degraders are valuable to compare to the results of the culturing experiments to determine if known degraders exist in the soil of SSFL.

Natural attenuation and remediation rates for dioxins in soils are tabulated in Table 3.5. Some studies indicate that chlorinated dioxins do not biodegrade in soil (Wilson et al. 1997), while others suggest biodegradation may be a viable means of natural attenuation (H. Suhara et al. 2003). For example, Wilson et al. (1997) reported no decrease in soil dioxin concentrations after 260 days of monitoring. Conversely, a microcosm study indicated 37-44\% removal of 2378-TeCDD at concentrations ranging from 1-100 ppm (Kearney, Woolson, and Ellingto 1972). Other studies reported dioxin reductions of 2 to $86 \%$. Clearly, the rates of biodegradation will depend on contaminants' chemical composition and environmental conditions.

Dehalogenation of higher chlorinated dioxins is very slow, and requires anaerobic environments for bioremediation by bacteria (Bunge et al. 2003). Estimations for halflives of dioxins in the soil range from 1-120 years depending on the type of compound (Haglund 2007; Sinkkonen and Paasivirta 2000; Isosaan, Tuhkanen, and Vartiainen 2004). 
The large amounts of time needed to see significant decreases in dioxin concentration makes these studies difficult to conduct. Table 3.6 is a list of 37 unique microorganisms in 69 experiments that have been found to biodegrade dioxins. 
Table 3.5: Summary of reported rates of dioxin biodegradation under natural attenuation conditions. Initial and final concentrations are listed in in $\mathrm{mg} / \mathrm{kg}$ or $\mathbf{~ p p m}$, and kinetics have been calculated.

\begin{tabular}{|c|c|c|c|c|c|c|c|c|}
\hline \multirow{2}{*}{ Compound } & \multirow{2}{*}{ Matrix } & \multirow{2}{*}{$\begin{array}{l}\text { Lab/Field } \\
\quad(\mathrm{L} / \mathrm{F})\end{array}$} & \multicolumn{2}{|c|}{$\begin{array}{c}\text { Soil Dioxin } \\
\text { Concentration } \\
(\mathrm{mg} / \mathrm{kg}) \\
\end{array}$} & \multirow{2}{*}{$\begin{array}{l}\text { Length of } \\
\text { Study } \\
\text { (days) }\end{array}$} & \multicolumn{2}{|c|}{ Biodegradation Kinetics } & \multirow{2}{*}{ Reference } \\
\hline & & & Initial & Final & & $\begin{array}{c}\text { Percent } \\
\text { Degraded }\end{array}$ & $\begin{array}{c}\text { Rate of Degradation } \\
(\mathrm{mg} / \mathrm{kg} / \mathrm{day})\end{array}$ & \\
\hline $\mathrm{PeCDF}$ & $\begin{array}{l}\text { anaerobic } \\
\text { river } \\
\text { sediment }\end{array}$ & $\mathrm{F}$ & 100 & -- & -- & -- & -- & $\begin{array}{c}\text { Adriaens \& Grbicgalic, } \\
1994\end{array}$ \\
\hline $\mathrm{PeCDF}$ & $\begin{array}{c}\text { anaerobic } \\
\text { aquifer } \\
\text { sediments } \\
\end{array}$ & $\mathrm{F}$ & 100 & -- & -- & -- & -- & $\begin{array}{c}\text { Adriaens \& Grbicgalic, } \\
1994\end{array}$ \\
\hline $\mathrm{PCDF}$ & soil & $\mathrm{F}$ & 180 & 170 & 260 & 6 & $3.85 E-02$ & Wilson et al., 1997 \\
\hline PCDD & soil & $\mathrm{F}$ & 140 & 68 & 260 & 51 & $2.77 E-01$ & Wilson et al., 1997 \\
\hline $\mathrm{HeCDF}$ & $\begin{array}{l}\text { anaerobic } \\
\text { river } \\
\text { sediment }\end{array}$ & $\mathrm{F}$ & 100 & -- & -- & -- & -- & $\begin{array}{c}\text { Adriaens \& Grbicgalic, } \\
1994\end{array}$ \\
\hline $\mathrm{HeCDF}$ & $\begin{array}{c}\text { anaerobic } \\
\text { aquifer } \\
\text { sediments } \\
\end{array}$ & $\mathrm{F}$ & 100 & -- & -- & -- & -- & $\begin{array}{c}\text { Adriaens \& Grbicgalic, } \\
1994\end{array}$ \\
\hline HeCDD & $\begin{array}{l}\text { anaerobic } \\
\text { river } \\
\text { sediment } \\
\end{array}$ & $\mathrm{F}$ & 100 & -- & -- & -- & -- & $\begin{array}{c}\text { Adriaens \& Grbicgalic, } \\
1994\end{array}$ \\
\hline HeCDD & $\begin{array}{l}\text { anaerobic } \\
\text { aquifer } \\
\text { sediments } \\
\end{array}$ & $\mathrm{F}$ & 100 & -- & -- & -- & -- & $\begin{array}{c}\text { Adriaens \& Grbicgalic, } \\
1994\end{array}$ \\
\hline HCDD & $\begin{array}{l}\text { anaerobic } \\
\text { river } \\
\text { sediment } \\
\end{array}$ & $\mathrm{F}$ & 100 & -- & -- & -- & -- & $\begin{array}{c}\text { Adriaens \& Grbicgalic, } \\
1994\end{array}$ \\
\hline
\end{tabular}




\begin{tabular}{|c|c|c|c|c|c|c|c|c|}
\hline \multirow{2}{*}{ Compound } & \multirow{2}{*}{ Matrix } & \multirow{2}{*}{$\begin{array}{l}\text { Lab/Field } \\
\text { (L/F) }\end{array}$} & \multicolumn{2}{|c|}{$\begin{array}{c}\text { Soil Dioxin } \\
\text { Concentration } \\
(\mathrm{mg} / \mathrm{kg}) \\
\end{array}$} & \multirow{2}{*}{$\begin{array}{l}\text { Length of } \\
\text { Study } \\
\text { (days) }\end{array}$} & \multicolumn{2}{|c|}{ Biodegradation Kinetics } & \multirow{2}{*}{ Reference } \\
\hline & & & Initial & Final & & $\begin{array}{l}\text { Percent } \\
\text { Degraded }\end{array}$ & $\begin{array}{c}\text { Rate of Degradation } \\
(\mathrm{mg} / \mathrm{kg} / \mathrm{day})\end{array}$ & \\
\hline HCDD & $\begin{array}{c}\text { anaerobic } \\
\text { aquifer } \\
\text { sediments } \\
\end{array}$ & $\mathrm{F}$ & 100 & -- & -- & -- & -- & $\begin{array}{c}\text { Adriaens \& Grbicgalic, } \\
1994\end{array}$ \\
\hline TCDD & soil & $\mathrm{L}$ & 1 & 0.46 & 350 & 54 & $1.54 E-03$ & Kearney, et al., 1972 \\
\hline TCDD & soil & $\mathrm{L}$ & 1 & 0.46 & 350 & 54 & $1.54 E-03$ & Kearney, et al.,1972 \\
\hline TCDD & soil & $\mathrm{L}$ & 10 & 4.3 & 350 & 57 & $1.63 E-02$ & Kearney et al., 1972 \\
\hline TCDD & soil & $\mathrm{L}$ & 10 & 3.7 & 350 & 63 & $1.80 E-02$ & Kearney, et al.,1972 \\
\hline TCDD & soil & $\mathrm{L}$ & 100 & 44 & 350 & 56 & $1.60 E-01$ & Kearney, et al.,1972 \\
\hline TCDD & soil & $\mathrm{L}$ & 100 & 29 & 350 & 71 & $2.03 E-01$ & Kearney, et al.,1972 \\
\hline TCDD & soil & $\mathrm{L}$ & 91 & 81 & 90 & 11 & $1.11 E-01$ & Kearney, et al.,1972 \\
\hline TCDD & soil & $\mathrm{L}$ & 88 & 96 & 90 & -- & -- & Kearney, et al.,1972 \\
\hline TCDD & soil & $\mathrm{L}$ & 92 & 84 & 90 & 9 & $8.89 E-02$ & Kearney, et al.,1972 \\
\hline TCDD & soil & $\mathrm{L}$ & 98 & 90 & 90 & 8 & $8.89 E-02$ & Kearney, et al.,1972 \\
\hline TCDD & soil & $\mathrm{L}$ & 93 & 94 & 90 & -- & -- & Kao et al., 2001 \\
\hline TCDD & soil & $\mathrm{L}$ & 90 & 94 & 90 & -- & -- & Kao et al., 2001 \\
\hline TCDD & soil & $\mathrm{L}$ & 94 & 85 & 90 & 10 & $1.00 E-01$ & Kao et al., 2001 \\
\hline TCDD & soil & $\mathrm{L}$ & 90 & 88 & 90 & 2 & $2.22 E-02$ & Kao et al., 2001 \\
\hline TCDD & soil & $\mathrm{L}$ & 91 & 89 & 90 & 2 & $2.22 E-02$ & Kao et al., 2001 \\
\hline TCDD & soil & $\mathrm{L}$ & 95 & 90 & 90 & 5 & $5.56 E-02$ & Kao et al., 2001 \\
\hline TCDD & soil & $\mathrm{L}$ & 97 & 84 & 90 & 13 & $1.44 E-01$ & Kao et al., 2001 \\
\hline
\end{tabular}




\begin{tabular}{|c|c|c|c|c|c|c|c|c|}
\hline \multirow{2}{*}{ Compound } & \multirow{2}{*}{ Matrix } & \multirow{2}{*}{$\begin{array}{l}\text { Lab/Field } \\
\quad(\mathbf{L} / \mathbf{F})\end{array}$} & \multicolumn{2}{|c|}{$\begin{array}{c}\text { Soil Dioxin } \\
\text { Concentration } \\
(\mathrm{mg} / \mathrm{kg}) \\
\end{array}$} & \multirow{2}{*}{$\begin{array}{l}\text { Length of } \\
\text { Study } \\
\text { (days) }\end{array}$} & \multicolumn{2}{|c|}{ Biodegradation Kinetics } & \multirow{2}{*}{ Reference } \\
\hline & & & Initial & Final & & $\begin{array}{c}\text { Percent } \\
\text { Degraded }\end{array}$ & $\begin{array}{c}\text { Rate of Degradation } \\
(\mathrm{mg} / \mathrm{kg} / \text { day })\end{array}$ & \\
\hline TCDD & soil & $\mathrm{L}$ & 94 & 89 & 90 & 5 & $5.56 E-02$ & Kao et al., 2001 \\
\hline DF & soil & $\mathrm{L}$ & 1000 & 790 & 84 & 21 & $2.50 E+00$ & Wang \& Oyaizu, 2011 \\
\hline DD & soil & $\mathrm{L}$ & 20 & 17.6 & 84 & 12 & $2.86 E-02$ & Wang \& Oyaizu, 2011 \\
\hline 2,8-DCDF & soil & $\mathrm{L}$ & 20 & 17.6 & 84 & 12 & $2.86 E-02$ & Wang \& Oyaizu, 2011 \\
\hline 2,7-DCDD & soil & $\mathrm{L}$ & 20 & 17.6 & 84 & 12 & $2.86 E-02$ & Wang \& Oyaizu, 2011 \\
\hline $\begin{array}{l}2,4,8- \\
\text { TCDF }\end{array}$ & soil & $\mathrm{L}$ & 20 & 17.6 & 84 & 12 & $2.86 E-02$ & Wang \& Oyaizu, 2011 \\
\hline $\begin{array}{l}\text { 2,3,7,8- } \\
\text { TeCDD }\end{array}$ & $\begin{array}{l}\text { soil mixed } \\
\text { with } \\
\text { anaerobic } \\
\text { sludge }\end{array}$ & $\mathrm{L}$ & 0.096 & 0.013 & 90 & 86 & $9.22 E-04$ & Kao et al., 2001 \\
\hline $\begin{array}{l}1,2,4- \\
\text { TCDD }\end{array}$ & soil & $\mathrm{L}$ & 20 & 17.4 & 84 & 13 & $3.10 E-02$ & Wang \& Oyaizu, 2011 \\
\hline $\begin{array}{l}1,2,3,4- \\
\text { TCDD }\end{array}$ & $\begin{array}{c}\text { anaerobic } \\
\text { reservoir } \\
\text { sediment } \\
\text { slurries } \\
\end{array}$ & $\mathrm{L}$ & 15.5 & 7.9 & 120 & 49 & $6.33 E-02$ & Ahn et al., 2008 \\
\hline $\begin{array}{l}1,2,3,4- \\
\text { TCDD }\end{array}$ & soil & $\mathrm{L}$ & 20 & 16.2 & 84 & 19 & $4.52 E-02$ & Wang \& Oyaizu, 2011 \\
\hline 1-CDD & soil & $\mathrm{L}$ & 20 & 18 & 84 & 10 & $2.38 E-02$ & Wang \& Oyaizu, 2011 \\
\hline
\end{tabular}




\section{Notes for Table 3.5:}

${ }^{1}$ Initial measured concentration exceeds final measured concentration. No degradation rate could be extrapolated.

${ }^{2}$ No final concentration given in paper; contaminant half-lives provided. No calculations performed.

NS $=$ Not Specified

Table 3.6: Dioxin-degrading microorganisms cited in literature. Initial and final concentrations were used to determine

\section{degradation rates}

\begin{tabular}{|c|c|c|c|c|c|c|c|c|}
\hline \multirow{2}{*}{ Compound } & \multirow{2}{*}{$\begin{array}{l}\text { Microorganis } \\
\text { m Involved in } \\
\text { Degradation }\end{array}$} & \multicolumn{2}{|c|}{$\begin{array}{c}\text { Dioxin } \\
\text { Concentration } \\
(p p m) \\
\end{array}$} & \multirow{2}{*}{$\begin{array}{l}\text { Length } \\
\text { of } \\
\text { Study } \\
\text { (days) }\end{array}$} & \multirow{2}{*}{ Metabolites } & \multicolumn{2}{|c|}{ Biodegradation Kinetics } & \multirow{2}{*}{ Reference } \\
\hline & & Initial & Final & & & $\begin{array}{l}\text { Percent } \\
\text { Degraded }\end{array}$ & $\begin{array}{c}\text { Degradation } \\
\text { Rate (mg/kg/day) }\end{array}$ & \\
\hline $\begin{array}{l}\text { Dibenzo-p- } \\
\text { dioxin (DD) }\end{array}$ & $\begin{array}{l}\text { Rhodococcus } \\
\text { sp. strain p52 }\end{array}$ & 250 & 12.5 & 2.5 & $\begin{array}{c}\text { Ethers, } \\
\text { Hydrocarbons }\end{array}$ & 95 & 95 & Peng et al., 2013 \\
\hline DD & $\begin{array}{c}\text { Sphingomonas } \\
\text { wittichii RWI }\end{array}$ & 55 & 2.75 & 0.67 & NS & 95 & 78 & Wilkes et al., 1996 \\
\hline DD & $\begin{array}{c}\text { Beijerinckia sp. } \\
B 8 / 36\end{array}$ & 500 & 29.4 & 1 & $\begin{array}{c}1,2- \\
\text { dihydroxydibenz } \\
\text { o-p-dioxin }\end{array}$ & 94 & 471 & Klečka \& Gibson, 1980 \\
\hline DD & $\begin{array}{l}\text { Pseudomonas } \\
\text { veroniiPH-03 }\end{array}$ & 219 & 20.4 & 2.5 & Catechol & 91 & 79 & Hong et al., 2004 \\
\hline DD & $\begin{array}{c}\text { Rhodococcus } \\
\text { opacus SAO101 }\end{array}$ & 1 & 0.02 & 7 & $\begin{array}{l}\text { monohydroxy } \\
\text { dibenzo- p- } \\
\text { dioxin } \\
\end{array}$ & 98 & 0.014 & $\begin{array}{c}\text { Kimura \& Urushigawa, } \\
2001\end{array}$ \\
\hline
\end{tabular}




\begin{tabular}{|c|c|c|c|c|c|c|c|c|}
\hline \multirow{2}{*}{ Compound } & \multirow{2}{*}{$\begin{array}{c}\text { Microorganis } \\
\text { m Involved in } \\
\text { Degradation }\end{array}$} & \multicolumn{2}{|c|}{$\begin{array}{c}\text { Dioxin } \\
\text { Concentration } \\
(\mathbf{p p m}) \\
\end{array}$} & \multirow{2}{*}{$\begin{array}{l}\text { Length } \\
\text { of } \\
\text { Study } \\
\text { (days) }\end{array}$} & \multirow{2}{*}{ Metabolites } & \multicolumn{2}{|c|}{ Biodegradation Kinetics } & \multirow{2}{*}{ Reference } \\
\hline & & Initial & Final & & & $\begin{array}{c}\text { Percent } \\
\text { Degraded }\end{array}$ & $\begin{array}{c}\text { Degradation } \\
\text { Rate }(\mathrm{mg} / \mathrm{kg} / \mathrm{day})\end{array}$ & \\
\hline DD & $\begin{array}{l}\text { Klebsiella sp. } \\
\text { strain HL1 }\end{array}$ & 8.7 & 2.6 & 0.33 & NS & 70 & 18 & Fukuda et al., 2002 \\
\hline DD & $\begin{array}{c}\text { Sphingomonas } \\
\text { sp. HL7 }\end{array}$ & 8.7 & 0 & 0.17 & NS & 100 & 52 & Fukuda et al., 2002 \\
\hline DD & $\begin{array}{c}\text { Coprinellus } \\
\text { disseminatus } \\
\text { TUFC11148 } \\
\end{array}$ & NS & NS & 14 & NS & 99 & -- & Suhara et al., 2003 \\
\hline DD & $\begin{array}{c}\text { Coprinellus } \\
\text { disseminatus } \\
\text { TUFC34534 } \\
\end{array}$ & NS & NS & 14 & NS & 78 & -- & Suhara et al., 2003 \\
\hline DD & $\begin{array}{c}\text { Coprinellus } \\
\text { disseminatus } \\
\text { TUFC30081 } \\
\end{array}$ & NS & NS & 14 & NS & 62 & -- & Suhara et al., 2003 \\
\hline DD & $\begin{array}{c}\text { Pseudomonas } \\
\text { sp. Strain HH69 }\end{array}$ & 10 & NS & NS & $\begin{array}{c}\text { 1-hydroxy } \\
\text { dibenzo-p-dioxin }\end{array}$ & -- & -- & Harms et al., 1991 \\
\hline DD & $\begin{array}{l}\text { Phanerochaete } \\
\text { chrysosporium }\end{array}$ & 833 & 416 & 1 & $\begin{array}{c}2,3- \\
\text { dihydroxydibenz } \\
\text { o-p-dioxin }\end{array}$ & 50 & 417 & Joshi \& Gold, 1994 \\
\hline DD & $\begin{array}{c}\text { Cordyceps } \\
\text { sinensis strain } \\
\text { A } \\
\end{array}$ & 30 & 15 & 4 & $\begin{array}{c}\text { 2,2',4,5-tetrahy- } \\
\text { droxydiphenyl } \\
\text { ether,Catechol }\end{array}$ & 50 & 3.75 & Nakamiya et al., 2005 \\
\hline DD & $\begin{array}{c}\text { Staphylococcus } \\
\text { auriculans } \\
\text { DBF63 }\end{array}$ & 500 & NS & NS & $\begin{array}{l}\text { 1-hydro-1,la- } \\
\text { dihydroxy-D }\end{array}$ & -- & -- & Monna et al., 1993 \\
\hline 1-CDD & $\begin{array}{c}\text { Sphingomonas } \\
\text { wittichii } R W 1 \\
\end{array}$ & 55 & 22 & 0.67 & 3-Chlorocatechol & 60 & 49.5 & Wilkes et al., 1996 \\
\hline 1-CDD & $\begin{array}{c}\text { Beijerinckia sp. } \\
B 8 / 36\end{array}$ & 500 & 137 & 1 & None & 73 & 363 & Klečka \& Gibson, 1980 \\
\hline 1-CDD & $\begin{array}{l}\text { Pseudomonas } \\
\text { veronii PH-O3 }\end{array}$ & 219 & 25.6 & 2.5 & 3-Chlorocatechol & 88 & 77 & Hong et al., 2004 \\
\hline
\end{tabular}




\begin{tabular}{|c|c|c|c|c|c|c|c|c|}
\hline \multirow{2}{*}{ Compound } & \multirow{2}{*}{$\begin{array}{l}\text { Microorganis } \\
\text { m Involved in } \\
\text { Degradation }\end{array}$} & \multicolumn{2}{|c|}{$\begin{array}{c}\text { Dioxin } \\
\text { Concentration } \\
(\mathrm{ppm})\end{array}$} & \multirow{2}{*}{$\begin{array}{c}\text { Length } \\
\text { of } \\
\text { Study } \\
\text { (days) }\end{array}$} & \multirow{2}{*}{ Metabolites } & \multicolumn{2}{|c|}{ Biodegradation Kinetics } & \multirow{2}{*}{ Reference } \\
\hline & & Initial & Final & & & $\begin{array}{c}\text { Percent } \\
\text { Degraded }\end{array}$ & $\begin{array}{c}\text { Degradation } \\
\text { Rate }(\mathrm{mg} / \mathrm{kg} / \mathrm{day})\end{array}$ & \\
\hline 1-CDD & $\begin{array}{c}\text { Rhodococcus } \\
\text { opacus SAO101 }\end{array}$ & 1 & 0.08 & 7 & & 92 & 0.013 & $\begin{array}{c}\text { Kimura \& Urushigawa, } \\
2001\end{array}$ \\
\hline 2-CDD & $\begin{array}{c}\text { Sphingomonas } \\
\text { wittichii } R W 1 \\
\end{array}$ & 55 & 33 & 0.67 & 4-Chlorocatechol & 40 & 33 & Wilkes et al., 1996 \\
\hline 2-CDD & $\begin{array}{c}\text { Beijerinckia sp. } \\
B 8 / 36\end{array}$ & 500 & 136.5 & 1 & None identified & 73 & 364 & Klečka \& Gibson, 1980 \\
\hline 2-CDD & $\begin{array}{l}\text { Sphingomonas } \\
\text { sp. strain KAlb }\end{array}$ & NS & NS & 7 & NS & 96 & -- & $\begin{array}{c}\text { Nojiri, Habe, and Omori } \\
2001 \mathrm{a}\end{array}$ \\
\hline 2-CDD & $\begin{array}{l}\text { Pseudomonas } \\
\text { veronii } \mathrm{PH}-03\end{array}$ & 219 & 46.9 & 2.5 & 4-Chlorocatechol & 79 & 69 & Hong et al., 2004 \\
\hline 2-CDD & $\begin{array}{c}\text { Burkholderia } \\
\text { sp. JBI }\end{array}$ & 0.1 & 0.005 & 1 & 4-Chlorocatechol & 95 & 0.095 & Parsons et al., 1998 \\
\hline 2-CDD & $\begin{array}{l}\text { Klebsiella sp. } \\
\text { strain HL1 }\end{array}$ & 8.7 & 4.35 & 0.33 & NS & 50 & 13.1 & Fukuda et al., 2002 \\
\hline 2-CDD & $\begin{array}{l}\text { Sphingomonas } \\
\text { sp. HL7 }\end{array}$ & 8.7 & 0 & 0.17 & NS & 100 & 52 & Fukuda et al., 2002 \\
\hline 2-CDD & $\begin{array}{c}\text { Pseudomonas } \\
\text { resinovorans } \\
\text { CA10a } \\
\end{array}$ & 10 & 0.3 & 5 & 4-Chlorocatechol & 97 & 1.94 & $\begin{array}{c}\text { Nojiri, Habe, and Omori } \\
2001 \mathrm{a}\end{array}$ \\
\hline 2-CDD & $\begin{array}{c}\text { Terrabacter sp. } \\
\text { DBF63 }\end{array}$ & 10 & 2.5 & 0.83 & 4-Chlorocatechol & 75 & 9 & $\begin{array}{c}\text { Nojiri, Habe, and Omori } \\
2001 \mathrm{a}\end{array}$ \\
\hline 23-CDD & $\begin{array}{c}\text { Dehalococcoide } \\
\text { s sp. CBDB1 }\end{array}$ & 6.32 & 2.97 & 28 & 2-CDD & 53 & 0.012 & Bunge et al., 2003 \\
\hline 23-CDD & $\begin{array}{c}\text { Pseudomonas } \\
\text { sp. strain } \\
\text { CA10a } \\
\end{array}$ & 1 & 0.11 & 5 & $\begin{array}{c}4,5- \\
\text { Dichlorocatechol }\end{array}$ & 89 & 0.018 & $\begin{array}{l}\text { Nojiri, Habe, and Omori } \\
2001 \mathrm{a}\end{array}$ \\
\hline 23-CDD & $\begin{array}{l}\text { Sphingomonas } \\
\text { sp. strain KAlb }\end{array}$ & NS & NS & 7 & NS & 70 & -- & $\begin{array}{c}\text { Nojiri, Habe, and Omori } \\
2001 \mathrm{a}\end{array}$ \\
\hline
\end{tabular}




\begin{tabular}{|c|c|c|c|c|c|c|c|c|}
\hline \multirow{2}{*}{ Compound } & \multirow{2}{*}{$\begin{array}{l}\text { Microorganis } \\
\text { m Involved in } \\
\text { Degradation }\end{array}$} & \multicolumn{2}{|c|}{$\begin{array}{c}\text { Dioxin } \\
\text { Concentration } \\
(\mathbf{p p m}) \\
\end{array}$} & \multirow{2}{*}{$\begin{array}{l}\text { Length } \\
\text { of } \\
\text { Study } \\
\text { (days) }\end{array}$} & \multirow{2}{*}{ Metabolites } & \multicolumn{2}{|c|}{ Biodegradation Kinetics } & \multirow{2}{*}{ Reference } \\
\hline & & Initial & Final & & & $\begin{array}{c}\text { Percent } \\
\text { Degraded }\end{array}$ & $\begin{array}{c}\text { Degradation } \\
\text { Rate (mg/kg/day) }\end{array}$ & \\
\hline 23-CDD & $\begin{array}{c}\text { Beijerinckia } s p . \\
B 8 / 36\end{array}$ & 500 & 422.5 & 1 & None & 16 & 77.5 & Klečka \& Gibson, 1980 \\
\hline 23-CDD & $\begin{array}{c}\text { Rhodococcus } \\
\text { opacus SAO101 }\end{array}$ & 18.4 & 14.2 & 7 & NS & 23 & 0.06 & $\begin{array}{c}\text { Kimura \& Urushigawa, } \\
2001 \\
\end{array}$ \\
\hline 23-CDD & $\begin{array}{c}\text { Sphingomonas } \\
\text { sp. HL7 } \\
\end{array}$ & 10 & 0 & 0.33 & $\begin{array}{l}\text { 2-methyl-4H- } \\
\text { chroman-4-one }\end{array}$ & 100 & 30 & Fukuda et al., 2002 \\
\hline 23-CDD & $\begin{array}{c}\text { Terrabacter sp. } \\
\text { DBF63 }\end{array}$ & 10 & 2 & 0.83 & $\begin{array}{c}4,5- \\
\text { Dichlorocatechol }\end{array}$ & 80 & 9.6 & $\begin{array}{c}\text { Nojiri, Habe, and Omori } \\
2001 \mathrm{a}\end{array}$ \\
\hline 23-CDD & $\begin{array}{c}\text { Pseudomonas } \\
\text { sp. EE41 }\end{array}$ & 0.3 & 0.09 & 63 & NS & 70 & 0.0033 & Du et al., 2001 \\
\hline 2,7-DCDD & $\begin{array}{c}\text { Sphingomonas } \\
\text { wittichii } R W 1\end{array}$ & 50 & 23.5 & 4 & 4-Chlorocatechol & 53 & 6.63 & Hong et al. 2002 \\
\hline 2,7-DCDD & $\begin{array}{c}\text { Coprinellus } \\
\text { disseminatus } \\
\text { TUFC } 34534 \\
\end{array}$ & 0.3 & 0.25 & 14 & $\begin{array}{l}\text { monomethoxy- } \\
\text { DCDD }\end{array}$ & 17 & 0.0036 & Suhara et al., 2011 \\
\hline 2,7-DCDD & $\begin{array}{c}\text { Coprinellus } \\
\text { disseminatus } \\
\text { TUFC11148 } \\
\end{array}$ & 0.3 & 0.26 & 14 & $\begin{array}{l}\text { monomethoxy- } \\
\text { DCDD }\end{array}$ & 13 & 0.00286 & Suhara et al., 2011 \\
\hline 2,7-DCDD & $\begin{array}{c}\text { Coprinellus } \\
\text { micaceus } \\
\text { TUFC } 30081 \\
\end{array}$ & 0.3 & 0.15 & 14 & $\begin{array}{l}\text { monomethoxy- } \\
\text { DCDD }\end{array}$ & 50 & 0.011 & Suhara et al., 2011 \\
\hline 2,7-DCDD & $\begin{array}{l}\text { Phlebia } \\
\text { lindtneri }\end{array}$ & 12.6 & 8.8 & 14 & $\begin{array}{l}\text { monomethody- } \\
\text { diCDD } \\
\end{array}$ & 30 & 0.27 & Kamei \& Kondo, 2005 \\
\hline 2,7-DCDD & Erwinia sp. & 5 & 3.6 & 1 & NS & 28 & 1.4 & Liaw \& Srinivasan, 1990 \\
\hline
\end{tabular}




\begin{tabular}{|c|c|c|c|c|c|c|c|c|}
\hline \multirow{2}{*}{ Compound } & \multirow{2}{*}{$\begin{array}{l}\text { Microorganis } \\
\text { m Involved in } \\
\text { Degradation }\end{array}$} & \multicolumn{2}{|c|}{$\begin{array}{c}\text { Dioxin } \\
\text { Concentration } \\
(\mathrm{ppm}) \\
\end{array}$} & \multirow{2}{*}{$\begin{array}{l}\text { Length } \\
\text { of } \\
\text { Study } \\
\text { (days) }\end{array}$} & \multirow{2}{*}{ Metabolites } & \multicolumn{2}{|c|}{ Biodegradation Kinetics } & \multirow{2}{*}{ Reference } \\
\hline & & Initial & Final & & & $\begin{array}{c}\text { Percent } \\
\text { Degraded }\end{array}$ & $\begin{array}{c}\text { Degradation } \\
\text { Rate }(\mathrm{mg} / \mathrm{kg} / \mathrm{day})\end{array}$ & \\
\hline 2,7-DCDD & $\begin{array}{l}\text { Phanerochaete } \\
\text { chrysosporium }\end{array}$ & 2 & 1 & 27 & $\begin{array}{c}\text { 4-chloro-1,2- } \\
\text { benzoquinone 2- } \\
\text { hydroxy-1,4- } \\
\text { benzoquinone } \\
\end{array}$ & 50 & 0.037 & $\begin{array}{l}\text { Valli, Wariishi, \& Gold, } \\
1992\end{array}$ \\
\hline 2,7-DCDD & $\begin{array}{c}\text { Pseudomonas } \\
\text { sp. CA } 10\end{array}$ & 10 & 7.5 & 5 & & 25 & 0.5 & $\begin{array}{l}\text { Nojiri, Habe, and Omori } \\
2001 \mathrm{a}\end{array}$ \\
\hline 2,8-DCDD & $\begin{array}{c}\text { Rhodococcus } \\
\text { opacus SAO101 }\end{array}$ & 25.3 & 21.3 & 7 & NS & 16 & 0.57 & $\begin{array}{c}\text { Kimura \& Urushigawa, } \\
2001\end{array}$ \\
\hline $123-\operatorname{TrCDD}$ & $\begin{array}{c}\text { Dehalococcoide } \\
\text { s sp. CBDB1 }\end{array}$ & 28.9 & 11.6 & 57 & $\begin{array}{l}\text { 23-/13-DCDD, } \\
\text { 2-MCDD }\end{array}$ & 60 & 0.304 & Ballerstedt et al., 2004 \\
\hline 123-TrCDD & $\begin{array}{c}\text { Pseudomonas } \\
\text { resinovorans } \\
\text { CA10a }\end{array}$ & 1 & 0.61 & 5 & NS & 39 & 0.078 & $\begin{array}{l}\text { Nojiri, Habe, and Omori } \\
\text { 2001a }\end{array}$ \\
\hline 123-TrCDD & $\begin{array}{l}\text { Sphingomonas } \\
\text { wittichii } R W 1 \\
\end{array}$ & 10 & 8 & 5 & $\begin{array}{l}\text { trichlorotrihydro } \\
\text { xydiphenyl ether }\end{array}$ & 20 & 0.4 & Hong et al., 2002 \\
\hline $123-\operatorname{TrCDD}$ & $\begin{array}{l}\text { Pseudomonas } \\
\text { sp. EE41 }\end{array}$ & 1.2 & 0.83 & 21 & NS & 31 & 0.0176 & Du et al., 2001 \\
\hline 124-TrCDD & $\begin{array}{c}\text { Dehalococcoide } \\
\text { s sp. CBDB1 }\end{array}$ & 17.2 & 7.7 & 57 & $\begin{array}{l}\text { 13-DCDD, 2- } \\
\text { MCDD }\end{array}$ & 55 & 0.167 & Bunge et al., 2003 \\
\hline 237-TCDD & $\begin{array}{l}\text { Pseudomonas } \\
\text { sp. EE41 }\end{array}$ & 0.3 & 0.18 & 63 & NS & 40 & 0.0019 & Du et al., 2001 \\
\hline 237-TCDD & $\begin{array}{c}\text { Cordyceps } \\
\text { sinensis strain } \\
A\end{array}$ & 300 & 150 & 4 & MCC \& DCC & 50 & 37.5 & Nakamiya et al., 2005 \\
\hline 237-TCDD & $\begin{array}{l}\text { Sphingomonas } \\
\text { wittichii } R W 1\end{array}$ & 59 & 19.4 & 5 & 345-TCC & 67 & 7.9 & Nam et al., 2006 \\
\hline 1234-TeCDD & $\begin{array}{c}\text { Dehalococcoide } \\
\text { s sp. CBDB1 }\end{array}$ & 14.9 & 11.3 & 28 & $\begin{array}{c}\text { 124-TrCDD, 13- } \\
\text { /23-DCDD, 2- } \\
\text { MCDD }\end{array}$ & 24 & 0.13 & Bunge et al., 2003 \\
\hline
\end{tabular}




\begin{tabular}{|c|c|c|c|c|c|c|c|c|}
\hline \multirow{2}{*}{ Compound } & \multirow{2}{*}{$\begin{array}{l}\text { Microorganis } \\
m \text { Involved in } \\
\text { Degradation }\end{array}$} & \multicolumn{2}{|c|}{$\begin{array}{c}\text { Dioxin } \\
\text { Concentration } \\
(\mathbf{p p m})\end{array}$} & \multirow{2}{*}{$\begin{array}{l}\text { Length } \\
\text { of } \\
\text { Study } \\
\text { (days) }\end{array}$} & \multirow{2}{*}{ Metabolites } & \multicolumn{2}{|c|}{ Biodegradation Kinetics } & \multirow{2}{*}{ Reference } \\
\hline & & Initial & Final & & & $\begin{array}{c}\text { Percent } \\
\text { Degraded }\end{array}$ & $\begin{array}{c}\text { Degradation } \\
\text { Rate }(\mathrm{mg} / \mathrm{kg} / \mathrm{day})\end{array}$ & \\
\hline 1234-TeCDD & $\begin{array}{l}\text { D. ethanogenes } \\
\text { strain } 195\end{array}$ & 9.9 & 0.99 & 40 & $\begin{array}{c}\text { 124-TrCDD, 13- } \\
\text { DCDD }\end{array}$ & 90 & 0.22 & Fennell et al., 2004 \\
\hline 1234-TeCDD & $\begin{array}{l}\text { Sphingomonas } \\
\text { wittichii } R W 1\end{array}$ & 50 & 31.5 & 4 & $\begin{array}{c}3456-\mathrm{TCC}, 2- \\
\text { methoxy-3456- } \\
\text { tetrachloropheno } \\
1,45- \\
\text { dichlorocatechol } \\
\end{array}$ & 37 & 4.6 & Hong et al. 2002 \\
\hline 1234-TeCDD & $\begin{array}{l}\text { Phanerochaete } \\
\text { sordida } Y K-624\end{array}$ & 0.05 & 0.03 & 14 & NS & 40 & 0.0014 & Takada et al., 1996 \\
\hline 1234-TeCDD & $\begin{array}{l}\text { Pseudomonas } \\
\text { sp. EE41 }\end{array}$ & 1 & 0.62 & 21 & $\begin{array}{c}3456- \\
\text { tetrachlorocatech } \\
\text { ol } \\
\end{array}$ & 38 & 0.018 & Du et al., 2001 \\
\hline 1234-TeCDD & $\begin{array}{l}\text { Pseudomonas } \\
\text { veronii } \mathrm{PH}-03\end{array}$ & 322 & 264 & 5 & NS & 18 & 11.6 & Du et al., 2001 \\
\hline 1368-TeCDD & $\begin{array}{c}\text { Phlebia } \\
\text { brevispora }\end{array}$ & 16.1 & 8.1 & 90 & NS & 50 & 0.089 & Kamei et al. 2009 \\
\hline 2378-TeCDD & $\begin{array}{c}\text { Bacillus } \\
\text { megaterium }\end{array}$ & 0.005 & 0.002 & 244 & None identified & 60 & $1.23 \times 10^{-5}$ & Iii \& Matsumura, 1983 \\
\hline 2378-TeCDD & $\begin{array}{l}\text { Phanerochaete } \\
\text { chrysosporium }\end{array}$ & NS & NS & NS & NS & -- & -- & Bumpus et al. 1985 \\
\hline 2378-TeCDD & $\begin{array}{c}\text { Pseudomonas } \\
\text { sp. EE41 }\end{array}$ & 0.3 & 0.06 & 21 & NS & 80 & 0.0114 & Du et al., 2001 \\
\hline 2378-TeCDD & $\begin{array}{c}\text { Pseudallescheri } \\
\text { a boydii }\end{array}$ & 3.2 & 0.66 & 0.83 & & 79 & 3.05 & Ishii et al. 2009 \\
\hline 2378-TeCDD & $\begin{array}{c}\text { Pseudomonas } \\
\text { testosteroni } \\
\text { G1036 } \\
\end{array}$ & 0.3 & NS & NS & HTeCDD & -- & -- & Philippi et al., 1982 \\
\hline $\begin{array}{l}\text { 12378- } \\
\text { PeCDD }\end{array}$ & $\begin{array}{c}\text { Dehalococcoide } \\
\text { s sp CBDB1 }\end{array}$ & NS & NS & 84 & $\begin{array}{l}\text { 2378-TCDD, } \\
\text { DCDD, 237- } \\
\text { TrCDD }\end{array}$ & 75 & -- & Bunge et al., 2003 \\
\hline
\end{tabular}




\begin{tabular}{|c|c|c|c|c|c|c|c|c|}
\hline \multirow{2}{*}{ Compound } & \multirow{2}{*}{$\begin{array}{l}\text { Microorganis } \\
\text { m Involved in } \\
\text { Degradation }\end{array}$} & \multicolumn{2}{|c|}{$\begin{array}{c}\text { Dioxin } \\
\text { Concentration } \\
(\mathrm{ppm})\end{array}$} & \multirow{2}{*}{$\begin{array}{l}\text { Length } \\
\text { of } \\
\text { Study } \\
\text { (days) }\end{array}$} & \multirow{2}{*}{ Metabolites } & \multicolumn{2}{|c|}{ Biodegradation Kinetics } & \multirow{2}{*}{ Reference } \\
\hline & & Initial & Final & & & $\begin{array}{c}\text { Percent } \\
\text { Degraded }\end{array}$ & $\begin{array}{c}\text { Degradation } \\
\text { Rate (mg/kg/day) }\end{array}$ & \\
\hline $\begin{array}{l}123478- \\
\text { HCDD }\end{array}$ & $\begin{array}{c}\text { Mixed culture } \\
\text { containing D. } \\
\text { ethanogenes } \\
195 \\
\end{array}$ & 186 & 167.4 & 200 & $\begin{array}{l}\text { 1378-/1248- } \\
\text { TCDD }\end{array}$ & 10 & 0.093 & Liu \& Fennell, 2008 \\
\hline $\begin{array}{l}123478- \\
\text { HCDD }\end{array}$ & $\begin{array}{c}\text { Sphingomonas } \\
\text { RWI }\end{array}$ & 50 & 36 & 5 & $\begin{array}{c}\text { TeCC and 2- } \\
\text { methoxy-3456- } \\
\text { tetrachloropheno } \\
1\end{array}$ & 28 & 2.8 & Nam et al., 2006 \\
\hline $\begin{array}{l}123478- \\
\text { HCDD }\end{array}$ & $\begin{array}{l}\text { Phanerochaete } \\
\text { sordida } Y K-624\end{array}$ & 0.05 & 0.012 & 14 & NS & 76 & 0.0027 & Takada et al., 1996 \\
\hline OCDD & $\begin{array}{c}\text { Cordyceps } \\
\text { sinesis strain A }\end{array}$ & 300 & 150 & 4 & $\begin{array}{l}\text { MCC, DiCC, } \\
\text { TrCC }\end{array}$ & 50 & 37.5 & Nakamiya et al., 2005 \\
\hline
\end{tabular}




\subsection{Methods}

\subsection{Soil Sample Site Selection}

Soil samples were collected from 30 locations within Area IV. This number of sample sites was selected after consideration of statistical needs and budget. Three sets of 10 soil samples were collected from Area IV locations. Sample sets of 10 were taken for both PCBs and dioxins (20 samples between the 2 sets). High TPH and PAHs concentrations were co-located throughout the site so those sample sets were combined into a single set of 10 samples. Thus, a total of 30 soil samples were collected for this part of the study. For each set sample sites were selected to cover a range of concentrations of each class of COI from low to moderately high concentrations. This was done so that the TRFLP analysis could be performed on set of samples that spanned a range of COI concentrations. The hope was that an increase in concentration of a particular COI could be related to the increase in a specific peak indicating a specific microbe or group of microbes. Moderately high COI concentrations are COI concentrations high enough to allow for reliable analytical detection of the COIs, but not so high as to be toxic to the microorganisms, as determined by the literature review. Target maximum soil concentrations of the bioremediation treatability study COIs are:

- TPH: approximately $500 \mathrm{ppm}$ or less

- PAH: approximately 6 ppm or less

- PCB: approximately $20 \mathrm{ppb}$ or less

- Dioxin: approximately $6 \mathrm{ppb}$ or less

These soil samples were used for isolating Area IV bacteria and fungi that may be capable of biodegrading the particular COI of moderately high concentrations. These 
same soil samples were used for extraction of DNA for TRFLP and metagenomics assays. The locations and COI concentrations for these soil samples are shown in the results chapter (Figure 5.1 and Table 5.1). Concentrations of COCs as well as other factors discussed below was used in the analysis of the TRFLP data. Chemical analysis of COI degradation products was scheduled to be performed but was cut due to lack of funding. Instead, historical data from a chemical characterization on the site performed by CDM Smith in November of 2011 was used.

The qPCR analysis was performed on 2 soil samples, a composite sample from a companion experiments being performed at SSFL and one sample from the set of 30 described above. Sample 1 was from sample site D03, and was selected because it had the highest concentration of chlorinated compounds out of the samples that were received within 48 hours of shipping to Microbial Insights for analysis. Sample 2 was a composite soil sample that came from 3 sample sites. These samples were also part of the two companion studies for bioremediation and phytoremediation on the site. Approximately 5 gallons of soil was collected from these sites and sifted. The soil was then combined into buckets and stored for 3 months in the lab before being collected for qPCR analysis. In contrast, sample 1 (from D03) was a fresh soil sample and was sent to the lab within 48 hours of collection.

\subsection{DNA Extraction Protocol}

This DNA extraction protocol was used for several segments of the experiments. Using the Power Soil DNA Extraction $\mathrm{Kit}^{\circledR}$ (MoBio, Carlsbad, CA), 1 gram of soil sample was added to 2-mL PowerBead® Tubes (MoBio, Carlsbad, CA). The PowerBead® Tube contains a anthracite beads that help break down cell membranes and buffer that 
disperses the soil particles, dissolves humic acids, and protects nucleic acids from degradation. For pure culture samples approximately $20 \mu \mathrm{L}$ of biomass from suspension was added. For each sample this was done 3 times in 3 tubes. The products of these 3 tubes were combined later. If at the end there was less then $10 \mathrm{ng} / \mu \mathrm{L}$ of DNA in the final solution, then the whole procedure was repeated with 6 tubes. For samples that were reextracted due to not enough DNA from the first extraction only 1/4 gram of soil was used in 6 replicates. This allows more volume of reagent per gram of soil, allowing for better extraction efficiency. Samples were vortexed on high for $5 \mathrm{~s}$. Solution C1 in the extraction kit was checked to make sure there was no precipitation. If there was precipitation the solution was heated to $60^{\circ} \mathrm{C}$ until dissolved before use. Solution $\mathrm{C} 1$ contains sodium dodecyl sulfate (SDS) and other disruption agents required for complete cell lysis. In addition to aiding in cell lysis, SDS is an anionic detergent that breaks down fatty acids and lipids associated with the cell membrane of many organisms. $60 \mu \mathrm{L}$ of Solution C1 was added to each sample and inverted. A Fast Prep machine (Thermo Scientific) was then used to mechanically mix the soil in the tubes. The tubes were placed in a rack and clamped down. The machine then moves the rack much like a paint can mixer to introduce mechanical shaking. This step is critical for complete homogenization and cell lysis. Cells lysed by a combination of chemical agents and mechanical shaking introduced by the Fast Prep machine. By randomly shaking the anthracite beads in the presence of disruption agents, collision of the beads with one another and with microbial cells causes the cells to break open. For soil samples, the Fast Prep machine was used to mix the samples at $5 \mathrm{~m} / \mathrm{s}$ for $45 \mathrm{~s}$. For pure culture samples, the Fast Prep was used to mix samples at $4.5 \mathrm{~m} / \mathrm{s}$ for $30 \mathrm{~s}$. Tubes were then centrifuged at 10,000 x g for $30 \mathrm{~s}$. 
Between 400 to $500 \mu \mathrm{L}$ of supernatant was transferred to a clean 2-mL microcentrifuge tube. Supernatant was occasionally dark in appearance and still contained some soil particles, particularly for clay soils. Subsequent steps in the protocol removed both soil particles and coloration of the mixture. If less than $400 \mu \mathrm{L}$ of supernatant was produced then the samples were centrifuged again and the remaining supernatant was transferred. Again, this happened only occasionally and only with the clay soils. After centrifuging, $250 \mu \mathrm{L}$ of Solution $\mathrm{C} 2$ was added to the samples and vortexed for $5 \mathrm{~s}$. These samples were then incubated in the freezer for $10-15 \mathrm{~min}$. Solution $\mathrm{C} 2$ contains a reagent to precipitate non-DNA organic and inorganic material including humic acid, cell debris, and proteins. After $15 \mathrm{~min}$ the samples were centrifuged for $1 \mathrm{~min}$ at $10,000 \mathrm{x} \mathrm{g}$. Avoiding the pellet mass in the bottom of the tube, up to $600 \mu \mathrm{L}$ of supernatant was transferred to a clean microcentrifuge tube. The pellet at this point contained non-DNA organic and inorganic material including humic acid, cell debris, and proteins. For the best DNA yields and quality, transferring any of the pellet with the supernatant was avoided with careful pipetting. $200 \mu \mathrm{L}$ of solution C3 were added to each sample and vortexed for $5 \mathrm{~s}$. Samples were cooled in the freezer for 10-15 min. Solution C3 is a second reagent to precipitate additional non-DNA organic and inorganic material including humic acid, cell debris, and proteins. Samples were again centrifuged for $1 \mathrm{~min}$ at $10,000 \times \mathrm{g}$. Up to $750 \mu \mathrm{L}$ of supernatant was transferred to a clean microcentrifuge tube. The pellet at this point contains additional non-DNA organic and inorganic material including humic acid, cell debris, and proteins. $1.2 \mathrm{~mL}$ of Solution $\mathrm{C} 4$ were added to the supernatant and vortexed for $5 \mathrm{~s}$. Solution $\mathrm{C} 4$ is a high-concentration salt solution. Since DNA binds tightly to silica at high salt concentrations, this solution will adjust the salt 
concentrations to allow binding of DNA, but not non-DNA organic and inorganic material that may still be present at low levels, to the spin filters used in the next step. Approximately $675 \mu \mathrm{L}$ of sample were loaded onto a spin filter and centrifuge at 10,000 $\mathrm{x} \mathrm{g}$ for $1 \mathrm{~min}$. Permeate was discarded into the Mo Bio waste container and $675 \mu \mathrm{L}$ more supernatant was loaded on the spin filter and centrifuged at $10,000 \mathrm{x} \mathrm{g}$ for $1 \mathrm{~min}$. Load the remaining supernatant onto the spin filter and centrifuge at $10,000 \mathrm{xg}$ for $1 \mathrm{~min}$. This was repeated until all the supernatant from all replicate tubes was filtered through the same filter. A total of three loads for each tube processed are required.

DNA is selectively bound to the silica membrane in the spin filter device in the high salt solution. Almost all contaminants pass through the filter membrane, leaving only the desired DNA behind. Once the replicates are all combined onto one filter $500 \mu \mathrm{L}$ of Solution C5 was added and centrifuged for $30 \mathrm{~s}$ at 10,000 x g. Solution C5 is an ethanolbased wash solution used to further clean the DNA that is bound to the silica filter membrane in the spin filter. This wash solution removes residues of salt, humic acid, and other contaminants while allowing the DNA to stay bound to the silica membrane. The permeate was discarded. The permeate was just non-DNA organic and inorganic waste removed from the silica spin filter membrane by the ethanol wash solution. Samples were then centrifuged again for $1 \mathrm{~min}$. This second spin removes residual Solution C5 (ethanol wash solution). It is critical to remove all traces of wash solution because the ethanol in C5 can interfere with many downstream steps such as PCR, restriction digests and gel electrophoresis (Complete Genomics 2013). The filter was carefully moved to a clean microcentrifuge tube. Then $100 \mathrm{~mL}$ of nano-pure, PCR-grade water was added to the center of the white filter membrane and incubated for $15 \mathrm{~min}$. As the water passes 
through the silica membrane, DNA is released because it only stays bound to the silica spin filter membrane in the presence of high salt concentration. Samples were centrifuged at $10,000 \mathrm{x} \mathrm{g}$ for $30 \mathrm{~s}$. Filters were removed and discarded. DNA was quantified using the Spectradrop spectrometer. A 4- $\mu \mathrm{L}$ (1-mm) slide cover was used. DNA was stored in a freezer at around $-20^{\circ} \mathrm{C}$ ) until use.

\subsection{TRFLP Analysis of Soil Samples}

DNA was extracted from each soil sample using the MoBio soil DNA extraction kit as described above and then prepped for PCR as follows.

\section{PCR for TRFLP}

Each PCR reaction well contained $10 \mu \mathrm{L}$ of the sample's DNA extracted with the MoBio system and $40 \mu \mathrm{L}$ of master mix. For bacterial TRF the master mix contained $10 \mu \mathrm{L} 5 \mathrm{X}$ Buffer, $3 \mu \mathrm{L}$ dNTPs (10mM, 2.5mM of each, A,T,C,G), $2 \mu \mathrm{L}$ BSA (20ug/mL ), $7 \mu \mathrm{L}$ $\mathrm{MgCl}_{2}(25 \mathrm{mM}), 1 \mu \mathrm{L}$ labeled $8 \mathrm{dF}(10 \mathrm{uM}) \quad$ (AGAGTTTGTTCMTGGCTCAG), $0.3 \mu \mathrm{L}$ AmpliTaq Gold $(5 \mathrm{U} / \mu \mathrm{L})$ and enough water to bring the total volume up to $50 \mu \mathrm{L}$ per sample. For fungal TRF the master mix contained $10 \mu \mathrm{L} 5 \mathrm{X}$ Buffer, $2 \mu \mathrm{L}$ dNTPs (10mM, 2.5mM of each, A,T,C,G), $5 \mu \mathrm{L} \mathrm{MgCl}_{2}(25 \mathrm{mM}), 1 \mu \mathrm{L}$ labeled ITS1F (10 uM) (GTATTACCGCGGCTGCTGG), $1 \mu \mathrm{L}$ ITS4 (10uM), $0.3 \mu \mathrm{L}$ AmpliTaq Gold (5U/ $\mu \mathrm{L})$ and enough water to bring the total volume up to $50 \mu \mathrm{L}$ per sample. Two control reactions were used for each PCR run. These controls included a closed negative (master mix, no DNA, not opened outside PCR room), and a positive (DNA known to amplify with PCR conditions). The positive controls were E. coli for bacterial samples and Pichia farinose for fungal samples. The PCR machine was set to the following cycling parameters for bacterial PCR: $94^{\circ} \mathrm{C}$ for $10 \mathrm{~min}$, then 30 cycles $\left(94^{\circ} \mathrm{C}\right.$ for $1 \mathrm{~min}, 46.5^{\circ} \mathrm{C}$ 
for $1 \mathrm{~min}, 72^{\circ} \mathrm{C}$ for $2 \mathrm{~min}$ ), then $72^{\circ} \mathrm{C}$ for $10 \mathrm{~min}$ and then $4^{\circ} \mathrm{C}$ soak until the samples were removed from the machine. For fungal PCR the following parameters were used: $94^{\circ} \mathrm{C}$ for $10 \mathrm{~min}$, then 13 more cycles $\left(95^{\circ} \mathrm{C}\right.$ for $35 \mathrm{~s}, 55^{\circ} \mathrm{C}$ for $55 \mathrm{~s}, 72^{\circ} \mathrm{C}$ for $\left.45 \mathrm{~s}\right)$, then 13 cycles $\left(95^{\circ} \mathrm{C}\right.$ for $35 \mathrm{~s}, 55^{\circ} \mathrm{C}$ for $55 \mathrm{~s}, 72^{\circ} \mathrm{C}$ for $\left.2 \mathrm{~min}\right)$, then 9 cycles of $\left(95^{\circ} \mathrm{C}\right.$ for $35 \mathrm{~s}$, $55^{\circ} \mathrm{C}$ for $55 \mathrm{~s}, 72^{\circ} \mathrm{C}$ for $3 \mathrm{~min}$ ), then $72^{\circ} \mathrm{C}$ for $10 \mathrm{~min}$ and then $4^{\circ} \mathrm{C}$ soak until the samples were removed from the machine. After the first round of PCR, gels were run to ensure the DNA was replicated. $5 \mu \mathrm{L}$ of PCR product were added to each well on a $1.5 \%$ agrose gel. The gels ran for $20 \mathrm{~min}$ at 100 Volts and $400 \mathrm{~mA}$. DNA was visualized with ethidium bromide. For samples that had successful PCR, two more rounds of PCR were done with gels to confirm DNA replication for each. For samples that had unsuccessful PCR DNA was re-extracted from culture or soil.

After the gel was visualized and it was confirmed that the PCR was successful 2 more PCR runs for each sample were done. These replicates were done to ensure even replication of all DNA in the samples because they are community samples.

\section{PCR Clean Up}

Using the PCR Ultra-Clean kit (MoBio, Carlsbad, CA), 5 volumes SpinBind ${ }^{\circledR}$ solution were added to each well and pipeted up and down to mix. Sample replicates were then combined into a spin filter and centrifuged for $30 \mathrm{sec}$ at $10,000 \mathrm{x}$ g. Permeate was discarded into MoBio waste container. $300 \mathrm{~mL}$ of SpinClean ${ }^{\circledR}$ buffer was added to each spin filter and centrifuged for $30 \mathrm{sec}$ at $10,000 \mathrm{x}$ g. Permeate was discarded into MoBio waste container. Spin filters were centrifuged for $120 \mathrm{sec}$ at $10,000 \mathrm{x} \mathrm{g}$ to remove any remaining fluid. Spin filters were transferred to clean $2.0-\mathrm{mL}$ collection tubes. $60 \mathrm{~mL}$ of PCR water was added to the spin filter and incubated for $10 \mathrm{~min}$. Samples were 
centrifuged for $60 \mathrm{sec}$ at $10,000 \mathrm{x}$ g. Spin filters were discarded. DNA was quantified with the Spectradrop spectrometer. DNA was stored at $-20^{\circ} \mathrm{C}$.

\section{DNA Digest}

Based on the concentration of DNA in each sample, $30 \mathrm{ng}$ of DNA was added to each well in a 96 well CEQ plate. These clear plastic plates were used in the CEQ 8000 machine in a later step. 5-10 ng of either E. coli or Pichia digest standard were used as controls. For bacterial samples $1.0 \mu \mathrm{L}$ DpnII (10,000 U/mL ) and $4 \mu \mathrm{L}$ buffer were added per reaction. PCR grade water was added to bring the volume to $40 \mu \mathrm{L}$. For fungal samples $1.0 \mu \mathrm{L}$ of HaeIII (10,000 U/mL ) and $4 \mu \mathrm{L}$ buffer were added per reaction. PCRgrade water was added to bring the volume to $40 \mu \mathrm{L}$. The samples were placed in the PCR machine for 4 hours @ $37^{\circ} \mathrm{C}$ then cycled to either $65^{\circ} \mathrm{C}$ for $\mathrm{DpnII}, 65^{\circ} \mathrm{C}$ for HhaI, or $80^{\circ} \mathrm{C}$ for $\mathrm{HaeIII}$ for $20 \mathrm{~min}$ to deactivate the enzyme and finally to $4^{\circ} \mathrm{C}$ until they were removed from the machine. Samples were stored at $-20^{\circ} \mathrm{C}$ until ready for ethanol precipitation.

\section{Ethanol Precipitation}

$100 \mathrm{~mL}$ (2.5 x digest volume) of cold $95 \%$ ethanol and $2 \mathrm{~mL} \mathrm{3M} \mathrm{NaAc} \mathrm{pH4.6} \mathrm{(5 \%} \mathrm{digest}$ volume $)$ and $1 \mu \mathrm{L}$ glycogen $(20 \mathrm{mg} / \mathrm{mL})$ were added to each digest sample in the CEQ plate. With the caps on, the plate was inverted 5 times to mix. The samples were then placed in the $-20^{\circ} \mathrm{C}$ freezer for $30 \mathrm{~min}$. Samples were then centrifuged for $15 \mathrm{~min}$ at 5300 RPM to pellet DNA (program 2). Prompt removal of samples from centrifuge will ensure minimal loss of sample. Samples were then inverted once to remove ethanol. $100 \mu \mathrm{L}$ of cold $70 \%$ ethanol was then added to each sample. Samples were then centrifuged for 5 min at 5300 RPM (program 3). Ethanol was removed by inverting the PCR tray once on a 
paper towel. The CEQ plate was inverted on top of a paper towel, placed back in the centrifuge still inverted, and centrifuged for 1 min. @ 700 RPM to dry the pellet (program 4). DNA was stored in the $-20^{\circ} \mathrm{C}$ freezer until ready to proceed to CEQ8000 preparation.

\section{CEQ 8000 Sample Preparation}

The CEQ 8000 (Beckman Coulter, Brea, CA) is a genetic analysis system that performs fragment analyses on digested DNA samples. It measures the relative quantity of fragments for each fragment length and produces a chromatograph. A master mix was made of $20 \mu \mathrm{L}$ formamide and $0.25 \mu \mathrm{L}$ 600-base-pair standard per reaction. $20 \mu \mathrm{L}$ of the master mix was added to each tube. One drop of mineral oil was added to the top of each well to prevent sample evaporation. CEQ program was set up with each sample's name. PCR grade water was added to the tray inside the CEQ. The CEQ was run. The appropriate PPE was used during DNA extractions, PCR, PCR clean up, DNA Digest, and operating the CEQ.

\section{TRFLP Analysis}

Bacterial and fungal TRFLP data were analyzed separately. Fragment data produced by the CEQ 8000's fragment analysis was transferred to an excel spreadsheet where it was truncated to $1 \%$ using a macro program. This removes all peaks that are less then $1 \%$ of the largest peak, effectively removing the "noise" in the data. A similarity matrix was run on the data in Primer 5. Chemical data for all COIs for each site was added as "sample data". A non-metric multi dimensional scaling analysis (MDS) was performed on the similarity matrix. This method determines non-parametric monotonic relationships between the similarities within the similarity matrix. Non-metric refers to the fact that the 
data does not belong to any specific distribution. Results were visualized in two dimensional scatter plots. The following factors were added to each sample site in the similarity matrix: COI series, location, soil type, presence of TPH, presence of PAHs, presence of PCBs, and presence of dioxin. COI series was defined by which sample set the sample came from (i.e. TPH/PAH, dioxin, or PCBs). The categories were $\mathrm{T}$ for TPH/PAH, P for PCBs, and D for dioxins. Location was selected based on the map of Area IV with the 30 sample sites overlaid. The 7 most northern sites were designated north and the 9 southern most sample sites were designated south. The remaining samples were labeled central. Soil type was designated based on visual observations of the soil. Categories included sandy, sandy loam, clay, clay loam, silt, and silt loam. Presence of TPH was determined by a threshold of $350 \mathrm{ppm}$. Presence of PAHs was determined by a threshold of $2.5 \mathrm{ppm}$. Presence of PCBs was determined by a threshold of $450 \mathrm{ppb}$. Presence of dioxin was determined by a threshold of $5 \mathrm{ppb}$. The concentrations of the respective contaminants were used to generate corresponding bubbles over each sample that indicated the concentration. Factors used in MDS analysis of TRFLP data are shown in the results section in Table 5.7.

In addition to the MDS analysis, the Primer 5 was also used to calculate the index multivariate dispersion (MVDISP) for each factor. This index is a measure of how similar two groups of samples are. It is analogous to a p value in statistical significance testing. In order to be significant the index of two groups must be between .05 and -.05 (Stobart et al. 2009). These indices provide a quantitative measurement of how similar two groups of samples are. 
TRFLP fragment data was also analyzed for correlations between specific peaks and COI concentrations using a correlation function in Excel. COI concentrations and fragment data for each sample was used in these correlations. Correlations between a particular fragment length and a COI were considered significant if the square of the correlation was above 0.4 .

Finally, the fragment lengths of microbes isolated in the culturing experiments were searched for in the TRFLP data. For each isolate the sequence data was imported to a Word document. The word search function was used to determine what the fragment length would be if digested with the restriction enzymes used for the TRFLP analysis. The enzyme DpnII was used on bacteria and HaeIII was used for fungi. These enzymes cut DNA at very specific sequences. Using a word search on the text file of the sequence data, a space was added in the middle of the first instance of that restriction sequence. A character count was then used to determine how long the DNA fragment would be if the restriction enzyme cut there. The fragment lengths were recorded for later comparison to TRFLP data.

For each isolate, the samples that contain its TRFLP signature as well as its relative abundance are presented in Table 5.4. Because of the possible error from the CEQ machine and the PCR digest, TRFLP peaks that were within 1 base pair of the cultured microbe's predicted fragment length were considered reported in this table.

\section{4 qPCR Analysis of Environmental Samples}

Two soil samples were used in the qPCR analysis. Sample 1 was from sample site D03, which had just been received from the site 2 days prior to being mailed to Microbial Insights. Sample 2 was a composite sample from 3 sample sites from the two companion studies for bioremediation and phytoremediation on the site. This composite sample had 
been sifted with a \#4 sieve $(4.75 \mathrm{~mm})$, and stored in a Tevlar® bag inside a 5-gallon bucket in the lab at room temperature for about 3 months. For each sample approximately $100 \mathrm{~g}$ of soil was placed in a whirlpak bag. These samples were shipped overnight on ice to Microbial Insights ${ }^{\circledR}$ in Knoxville, Tennessee.

Microbial Insights performed a QuantArray Petro analysis, as well as a CENSUS analysis for Dehalococcoides and biphenyl dioxygenase for each of the two samples. The QuantArray Petro includes the analysis of the following targets: benzene/toluene dioxygenase (TOD), toluene/benzene monooxygenases (RMO, RDEG), Phenol hydroxylase (PHE), ethylbenzene and isopropylbenzene dioxygenases (EDO, BPH4), naphthalene dioxygenases (NAH, NAG, PHN), MTBE-utilizing strain PM1, TBA monooxygenase, alkane monooxygenases, benzyl succinate synthase (BSS), benzene carboxylase $(\mathrm{ABC})$, naphthalene carboxylase, $(\mathrm{ANC})$, naphthylmethylsuccinate synthase (NMS), alkyl succinate synthase, benzoyl coenzyme A reductase (BCR), total bacteria (EBAC), and sulfate reducing bacteria (APS). These targets will be discussed in detail below.

QuantArray Petro Methods (from Microbial Insights®): This method uses qPCR as well, but combines it with microarrays to run numerous parallel reactions. A few nano liters of sample are added to each hole in a microarray slide. Each hole will carry out an individual qPCR reaction, and can target whatever gene is chosen. This allows for many genes to be targeted at the same time, leading to a greater amount of information about the sample. QuantArray uses discrete through-holes for each qPCR reaction which prevents compromising the reaction kinetics, which can be a problem for multiplex 
qPCR. qPCR reactions in this technique use primers and fluorescent markers like the CENSUS technique to select and count gene copies generated (Microbial Insights 2014a).

CENSUS Methods (from Microbial Insights®): CENSUS is a qPCR-based technique that uses fluorescent markers to count the number of gene copies generated in a PCR reaction. Each time a gene copy is made a fluorescent marker is released and measured with a detector. Primers are used to target specific genes to be duplicated. This technique is significantly more accurate then the traditional culturing methods, which can report less then $10 \%$ of a targeted microbe group leading to underestimating the population (Microbial Insights 2014b).

\section{qPCR Targets}

Per the recommendations of Microbial Insights, the analyses selected were the QuantArray® Petro and the Census: Dehalococcoides (DHC). The QuantArray® Petro included 18 targets for PHC and PAH degradation, including biphenyl dioxygenase which is involved in PCB biodegradation. Table 4.1 below summarizes the targets of the QuantArray® Petro assay and specifies the enzyme name, constituent attacked by the enzyme, constituent group, and if the enzyme is part of an aerobic or anaerobic process. The only specific bacterium target, Methylibium petroleiphilum PM1 (PM1), is one of the few bacteria have been isolated that can use methyl tertiary butyl ether (MTBE) or tetra butyl alcohol (TBA) as a growth substrate. This bacterium uses TBA monooxygenase (abbreviated TBA in Microbial Insight reports) to break down MTBE and TBA (Hanson, Ackerman, and Scow 1999). 
Table 4.1: Targets of QuantArray ${ }^{\circledR}$ Petro

\begin{tabular}{|c|c|c|c|c|}
\hline $\begin{array}{l}\text { Target } \\
\text { Name }\end{array}$ & Enzyme Name & $\begin{array}{c}\text { Constituent Attacked by } \\
\text { Enzyme }\end{array}$ & $\begin{array}{l}\text { Constituent } \\
\text { Group }\end{array}$ & $\begin{array}{c}\text { Aerobic/ } \\
\text { Anaerobic }\end{array}$ \\
\hline TOD & Benzene/Toluene Dioxygenase & Benzene/Toluene & TPH & Aerobic \\
\hline EDO & Ethylbenzene Dioxygenase & Ethylbenzene & $\mathrm{TPH}$ & Aerobic \\
\hline RMO & Toluene Monooxygenases & Toluene & $\mathrm{TPH}$ & Aerobic \\
\hline RDEG & Benzene Monooxygenases & Benzene & TPH & Aerobic \\
\hline TOL & Xylene/Toluene Monooxygenase & Xylene/Toluene & TPH & Aerobic \\
\hline PHE & Phenol hydroxylase & Phenol & $\mathrm{TPH}$ & Aerobic \\
\hline $\mathrm{BPH} 4$ & $\begin{array}{c}\text { Biphenyl/Isopropylbenzene } \\
\text { Dioxygenases }\end{array}$ & $\begin{array}{c}\text { Biphenyl/ } \\
\text { Isopropylbenzene }\end{array}$ & $\mathrm{TPH}$ & Aerobic \\
\hline $\begin{array}{l}\text { NAH, } \\
\text { NAG, } \\
\text { PHN }\end{array}$ & Naphthalene Dioxygenases & Naphthalene & PAHs & Aerobic \\
\hline PM1 & MTBE-utilizing strain PM1 & MTBE and TBA & TPH & Aerobic \\
\hline TBA & TBA Monooxygenase & TBA & TPH & Aerobic \\
\hline PHN & Phenanthrene Dioxygenase & Phenanthrene & PAHs & Aerobic \\
\hline ALK & Alkane Monooxygenases & Alkanes & PAHs & Aerobic \\
\hline BSS & Benzyl Succinate Synthase & Benzyl Succinate & $\mathrm{TPH}$ & Anaerobic \\
\hline$A B C$ & Benzene Carboxylase & Benzene & $\mathrm{TPH}$ & Anaerobic \\
\hline ANC & Naphthalene Carboxylase & Naphthalene & PAHs & Anaerobic \\
\hline ASSA & Alkyl Succinate Synthase & Alkyl Succinate & $\mathrm{TPH}$ & Anaerobic \\
\hline $\mathrm{BCR}$ & Benzoyl Coenzyme A Reductase & Benzoyl Coenzyme A & TPH & Anaerobic \\
\hline ASSA & Alklysuccinate Synthase & Alklysuccinate & PAHs & Anaerobic \\
\hline MNSSA & $\begin{array}{c}\text { Naphthylmethylsuccinate } \\
\text { Synthase }\end{array}$ & Naphthylmethylsuccinate & PAHs & Anaerobic \\
\hline APS & Sulfate Reducing Bacteria & N/A & N/A & Anaerobic \\
\hline EBAC & Total Bacteria & N/A & N/A & N/A \\
\hline
\end{tabular}

\subsection{Culturing and 16S Sequencing of Microbes from Contaminated Site Soil Media,}

\section{Stock Solutions of Model Chemicals}

The media used for isolating bacteria and fungi are depicted in Table 4.2. Bushnell Haas

Media is a carbon free mineral media, which contains no carbon food source for bacteria.

Carbon sources (such as COIs) can be added to it to determine if a microbe can survive

on that carbon source alone. This is the main medium used for isolation in this

experiment. Additionally, TSB was used to make liquid media to grow bacteria and YM 
media was used to grow fungi for enrichment cultures and cultivating biomass after initial isolation on spike Bushnell Haas media. 
Table 4.2: Composition and Recipes for Medias used in Culturing Experiments

\begin{tabular}{|c|c|c|c|c|c|c|c|}
\hline Medium & Components & $\begin{array}{l}\mathrm{g} / \mathrm{L} \text { of each } \\
\text { Component }\end{array}$ & $\begin{array}{c}\text { Final } \\
\mathrm{pH}\end{array}$ & $\begin{array}{l}\text { Main } \\
\text { Carbon } \\
\text { Source }\end{array}$ & $\begin{array}{l}\text { Medium } \\
\text { Phase }\end{array}$ & $\begin{array}{c}\text { Agar } \\
\text { Added } \\
\text { per 1L of } \\
\text { Water }(g)\end{array}$ & Reference \\
\hline $\begin{array}{l}\text { Bushnell } \\
\text { Haas }\end{array}$ & $\begin{array}{l}\text { Magnesium Sulfate } \\
\text { Calcium Chloride } \\
\text { Monopotassium } \\
\text { Phosphate } \\
\text { Dipotassium } \\
\text { Phosphate } \\
\text { Ammonium Nitrate } \\
\text { Ferric Chloride }\end{array}$ & $\begin{array}{c}0.2 \\
0.02 \\
1 \\
1 \\
1 \\
0.05\end{array}$ & $\begin{array}{c}7.0+1- \\
0.2\end{array}$ & none & Liquid & 20 & $\begin{array}{c}\text { (Sigma Aldrich } \\
\text { 2008) }\end{array}$ \\
\hline TSB & $\begin{array}{c}\text { Enzymatic Digest of } \\
\text { Casein } \\
\text { Enzymatic Digest of } \\
\text { Soybean Meal } \\
\text { Sodium Chloride } \\
\text { Dipotassium } \\
\text { Phosphate } \\
\text { Dextrose }\end{array}$ & $\begin{array}{c}17 \\
3 \\
5 \\
2.5 \\
2.5\end{array}$ & $\begin{array}{c}7.3+1- \\
0.2\end{array}$ & Dextrose & Liquid & 15 & (acumedia 2010a) \\
\hline YM & $\begin{array}{c}\text { Enzymatic Digest of } \\
\text { Gelatin } \\
\text { Malt Extract } \\
\text { Dextrose } \\
\text { Yeast Extract }\end{array}$ & $\begin{array}{c}5 \\
3 \\
10 \\
5\end{array}$ & $\begin{array}{c}6.2+/- \\
0.2\end{array}$ & Dextrose & Liquid & 15 & (acumedia 2010b) \\
\hline
\end{tabular}


To first isolate organisms that use these COIs for growth, solid or liquid carbon-free media or standard growth media were spiked with model compounds of the COIs. For liquid cultures grown in spiked media, cultures were subsequently plated out to form isolated colonies. From isolated colonies fresh, carbon-rich media was inoculated to grow enough biomass for DNA analyses. Instead of using every PAH, PCB, and dioxin for selecting for degrading organisms, model chemicals were selected based on the literature (Kyser, Hozalski, and Gulliver 2011; Jones, Arujo, and Rodgers 2012). These model compounds were used to screen for potential COI degraders by encouraging the growth of organisms that could degrade these less recalcitrant versions of the COIs. This both sped up the time needed to culture and also reduced the number of false positives, or cultured organisms that were not truly degraders. Once the microorganisms were isolated and identified they were compared to the literature to determine the likelihood that they are degraders.

\section{Table 4.3: Model Chemicals and Concentrations of Stock Solutions}

\begin{tabular}{|c|c|c|}
\hline COI & Model Chemical & $\begin{array}{c}\text { Concentration } \\
\text { of Stock } \\
\text { Solution }\end{array}$ \\
\hline TPH & Diesel Fuel \#2 & $0.3 \mathrm{M}$ \\
\hline PAH & Naphthalene & $0.3 \mathrm{M}$ \\
\hline PCBs & PCB \#1 & $0.03 \mathrm{M}$ \\
\hline Dioxins & Dibenzofuran & $0.03 \mathrm{M}$ \\
\hline
\end{tabular}

PCB \#1 was selected as a model for PCBs because it is only mono-chlorinated and therefore significantly easier to degrade than higher chlorinated PCBs (Beyer and Biziuk 2009). All COI stock solutions were made with acetone, which was used to measure and distribute the COIs and was then evaporated off. These solutions were made in 50-mL 
centrifuge tubes and stored with secondary containment in a closed cabinet. The concentrations of the stock solutions were $0.3 \mathrm{M}$ for diesel, $0.3 \mathrm{M}$ naphthalene, $0.03 \mathrm{M}$ PCB 1, and 0.03 M dibenzofuran. These stock solutions were checked to ensure that they were completely dissolved before pipetting out aliquots out for dilutions and media preparation. For each soil culture only one model chemical was used. For example, for samples that were from the dioxin set, and contained high dioxin concentration, microbes were cultured on media that contained dibenzofurans, but not naphthalene, No. 2 diesel, or PCB 1.

\section{Inoculation Procedures: Plate Cultures}

Plates were made from autoclaved TSA, YM, or Bushnell Haas agar (defined in Table 4.2). Dilutions of model chemicals were made from the stock solutions for each culturing experiment (See Culturing Experimental Design Tables below for details). Model chemicals did not dissolve in the liquid media, and so they would not be evenly distributed in the solid plates. Therefore the model chemicals were added to the top of the solid media. To each plate $5 \mathrm{~mL}$ of diluted model chemical solution was be added and spread evenly over the surface. It was assumed that the model chemicals, which were dissolved in acetone, would dissolve into the top portion of the plate, a volume of approximately $10 \mathrm{~mL}$. Once $5 \mathrm{~mL}$ of the appropriate dilution was on each plate the lids were propped up on top of each plate in a fume hood to allow the acetone to evaporate off. Plates were checked periodically to see if liquid remained on the surface of the plate. Once the liquid was gone, the plates were allowed to sit for another $12 \mathrm{hr}$ to ensure all acetone was volatilized. Negative control plates were run for each experiment to ensure all the acetone had been evaporated. These controls had clean acetone added to the top of 
the solid Bushnell Haas media, which was allowed to evaporate off for the same amount of time as the other plates. They were inoculated using soil from sample T01. This ensured that the acetone was completely volatilized off the plates and prevented the isolation of acetone degraders. Plates were then stored in a refrigerator until inoculation. To make an inoculum from the soil, approximately $1 \mathrm{~g}$ of soil was mixed into a $10-\mathrm{mL}$ centrifuge tube with $9 \mathrm{~mL}$ of autoclaved $1 \% \mathrm{NaCl}$ solution to create an inoculum. These tubes were vortexed for $10 \mathrm{~s}$ on high, then allowed to settle for $10 \mathrm{~min}$. The top $0.1 \mathrm{~mL}$ of the inoculum was pipetted onto the plate $\left(1 / 100^{\text {th }}\right.$ dilution). Sterile glass beads were then added to the plate and rolled around to spread the inoculum. After inoculation, the plates were incubated at $30^{\circ} \mathrm{C}$ until growth was observed (1-5 weeks) or the experiment ended at 5 weeks (See Culturing Experimental Design Table below). Isolated colonies that were grown on these plates were grown again in TSB or YM liquid media. DNA was then extracted from these new colonies. All plate cultures were performed in triplicate. Preparations for fungi and bacteria were identical except for the media used in the enrichment cultures. TSB was prepared for bacteria and YM was prepared for fungi enrichment cultures. Refer to Tables 4.3 and 4.4 for more details.

\section{Inoculation Procedures: Liquid/Enrichment Cultures}

Dilutions of model chemicals were made from the stock solutions for each culturing experiment (See Culturing Experimental Design Tables 5.3 and Table 5.4 below for details). Liquid cultures were prepared by adding the model chemicals in acetone to sterile 20 -mL test tubes and then evaporating off the acetone. This method was adapted from experiments done by Singer, Wong, and Crowley (2002). Negative control tubes were run for each experiment to ensure all the acetone had been evaporated. These 
controls had clean acetone added to the tube, which was allowed to evaporate off for the same amount of time as the other tubes. Bushnell Haas media was then added to the tubes. They were inoculated using soil from sample T01. This ensured that the acetone was completely volatilized off the tubes before media was added and prevented the isolation of acetone degraders. Concentrations of added model chemicals were based on the assumptions that $5 \mathrm{~mL}$ of diluted model chemical solution would be added to each tube and that the final volume of each culture was to be $10 \mathrm{~mL}$. Once $5 \mathrm{~mL}$ of the appropriate dilution was in each tube the rack was set with the caps off in a hood to allow the acetone to evaporate. Parafilm was suspended 1 inch over the tops of the tubes using an autoclaved scaffolding to minimize microbes falling into the tubes but also to allow enough air flow to evaporate the acetone in a reasonable amount of time. After the acetone was completely evaporated, $9 \mathrm{~mL}$ of Bushnell Haas carbon-free liquid medium or TSB or YM was added to each tube, depending on the culturing experiment. These tubes were placed in the incubator at $30^{\circ} \mathrm{C}$ for 1 week and then were checked for growth before inoculation. No growth was observed in any of the tubes before inoculation. To make an inoculum from the soil, approximately $1 \mathrm{~g}$ of soil was mixed into a $10-\mathrm{mL}$ centrifuge tube with $9 \mathrm{~mL}$ of autoclaved $1 \% \mathrm{NaCl}$ solution to create an inoculum. These tubes were vortexed for $10 \mathrm{~s}$ on high, then allowed to settle for $10 \mathrm{~min}$. Then the top 1 $\mathrm{mL}$ of the inoculum was added to each tube of medium $\left(1 / 10^{\text {th }}\right.$ dilution $)$. These tubes were incubated at $30^{\circ} \mathrm{C}$ until growth was observed or the experiment ended (6 to 11 weeks). If growth was observed then the culture was plated onto TSA and YM plates. Isolated colonies that were grown on these plates were grown again in TSB or YM liquid media. All liquid cultures were performed in triplicate. DNA was extracted from each 
enrichment culture using the Power Soil DNA Extraction Kit ${ }^{\circledR}$ from MoBio and the procedure described in section 3.3: DNA Extraction Protocol.

\section{Culturing Positive Control Organisms}

Bacterial and fungal positive control organisms that are known to degrade each model chemical were selected based on the literature review. These organisms were grown separately along with the microbes from the soil in every culturing experiment to determine if the concentration of the model chemicals had toxic effects on the microbes and to ensure that they could grow in the selected media. Table 4.4 summarizes these control organisms.

Table 4.4: Positive Control Organisms for Culturing Experiments

\begin{tabular}{|c|c|c|c|}
\hline $\begin{array}{l}\text { Model Compound } \\
\text { Chosen For }\end{array}$ & $\begin{array}{l}\text { Positive Control } \\
\text { Organism }\end{array}$ & $\begin{array}{l}\text { ATCC/DSMZ } \\
\text { Strain Number }\end{array}$ & Reference \\
\hline No. 2 Diesel & $\begin{array}{c}\text { Rhodocoicus rhodochrous } \\
\text { KUCC } 8801\end{array}$ & ATCC: 21198 & Sorkhoh et al. 1990 \\
\hline Naphthalene & $\begin{array}{c}\text { Paenibacillus } \\
\text { naphthalenovorans }\end{array}$ & $\begin{array}{c}\text { ATCC: BAA- } \\
206\end{array}$ & Daane et al. 2002 \\
\hline PCB \#1 & $\begin{array}{l}\text { Pseudomonas putida } \\
\text { KF715 }\end{array}$ & ATCC: 700837 & $\begin{array}{c}\text { Hayase, Taira, and } \\
\text { Furukawa } 1990\end{array}$ \\
\hline Dibenzofuran & $\begin{array}{c}\text { Sphingomonas wittichii } \\
\text { RW1 }\end{array}$ & DSM 6014 & T. R. Miller et al. 2010 \\
\hline Fungal Control 1 & $\begin{array}{l}\text { Phanerochaete } \\
\text { chrysosporium }\end{array}$ & ATCC 24725 & $\begin{array}{c}\text { Fernández-Sánchez et al. } \\
\text { 2001; Fernández-Luqueño } \\
\text { et al. 2011; Pérez- } \\
\text { Armendáriz et al. 2012; } \\
\text { Hammel, Kalyanaraman, } \\
\text { and Kirk 1986; Hammel et } \\
\text { al. } 1992\end{array}$ \\
\hline Fungal Control 2 & $\begin{array}{c}\text { Phanerochaete } \\
\text { chrysosporium strain for } \\
\text { Puerto Rico }\end{array}$ & N/A & N/A \\
\hline
\end{tabular}

Two strains of Phanerochaete were used, one bought from ATCC and one which was shipped on solid medium from Puerto Rico by Dr. Raul Cano (Cal Poly). All other 
microbes were ordered from ATCC and arrived freeze dried, and were revived based on the instruction received with the cultures. $0.5 \mathrm{~mL}$ of TSB or YM media was added to the freeze dried cultures. After $30 \mathrm{~min}$ the $0.5 \mathrm{~mL}$ culture broth was added to $5 \mathrm{~mL}$ of the appropriate medium and incubated for 5 days at $30^{\circ} \mathrm{C}$. Slants, plates and liquid cultures of these organisms were maintained throughout the experiment by re-plating onto the appropriate medium every 2 weeks. Colony morphology and visual observations of the cells under a microscope were used to confirm that the cultures were correct and pure. All model bacteria were grown on TSA or TSB, while fungi were grown on YM media (media described above in Table 4.2).

\section{DNA Extraction and Sequencing}

Once the microbes had been cultured in liquid media, their DNA was extracted using the MoBio DNA Power Soil Extraction Kit as described above (Section 3.3). Instead of using 1 gram of soil for each sample, approximately $1 \mathrm{~mL}$ of the bottom of the liquid culture was used in each PowerBead® tube (still used 3 PowerBead® tubes per sample). After the DNA was extracted and quantitated with the Spectradrop machine, PCR was done on each sample. Master mixes were prepared for both fungal and bacterial PCR.

For bacterial PCR the master mix contained $10 \mu \mathrm{L}$ 5X Buffer, $2 \mu \mathrm{L}$ dNTPs (10mM, 2.5mM of each, A,T,C,G), $2 \mu \mathrm{L} \mathrm{BSA} \mathrm{(20ug/mL} \mathrm{),} 4 \mu \mathrm{L} \mathrm{MgCl} 2$ (25mM), $1 \mu \mathrm{L} 8 \mathrm{dF}(10$ uM) (AGAGTTTGTTCMTGGCTCAG), $1 \mu \mathrm{L}$ of $1525 \mathrm{R}$ (10um), $23.7 \mu \mathrm{L}$ of $\mathrm{H} 2 \mathrm{O}$ and $0.3 \mu \mathrm{L}$ AmpliTaq Gold $(5 \mathrm{U} / \mu \mathrm{L}) .6 \mu \mathrm{L}$ of extracted DNA were added to each reaction. For bacterial PCR the following cycling parameters were used: $94^{\circ} \mathrm{C}$ for $2 \mathrm{~min}$, then 40 
cycles of $\left(94^{\circ} \mathrm{C}\right.$ for $30 \mathrm{~s}, 46.5^{\circ} \mathrm{C}$ for $30 \mathrm{~s}, 72^{\circ} \mathrm{C}$ for $\left.30 \mathrm{~s}\right)$, then $72^{\circ} \mathrm{C}$ for $7 \mathrm{~min}$ and then $4^{\circ} \mathrm{C}$ soak until the samples were removed.

For fungal PCR the master mix contained $10 \mu \mathrm{L}$ 5X Buffer, $2 \mu \mathrm{L}$ dNTPs (10mM, 2.5mM of each, A,T,C,G), $2 \mu \mathrm{L}$ BSA (20ug/mL ), $4 \mu \mathrm{L} \mathrm{MgCl2} \mathrm{(25mM),} 1 \mu \mathrm{L}$ ITS1F (10 uM) (GTATTACCGCGGCTGCTGG), $1 \mu \mathrm{L}$ ITS4 (10uM), $23.7 \mu \mathrm{L}$ of $\mathrm{H} 2 \mathrm{O}$ and $0.3 \mu \mathrm{L}$ AmpliTaq Gold (5U/ $\mu \mathrm{L}) .6 \mu \mathrm{L}$ of extracted DNA were added to each reaction. Two control reactions were used for each PCR run. These controls included a closed negative (master mix, no DNA, not opened outside PCR room), and a positive (DNA known to amplify with PCR conditions). The positive controls are E. Coli for bacterial samples and Pichia farinose for fungal samples. For Fungal PCR the following cycling parameters were used: $94^{\circ} \mathrm{C}$ for $10 \mathrm{~min}$, then 13 cycles of $\left(95^{\circ} \mathrm{C}\right.$ for $35 \mathrm{~s}, 55^{\circ} \mathrm{C}$ for $55 \mathrm{~s}, 72^{\circ} \mathrm{C}$ for 45 s), then 13 cycles of $\left(95^{\circ} \mathrm{C}\right.$ for $35 \mathrm{~s}, 55^{\circ} \mathrm{C}$ for $55 \mathrm{~s}, 72^{\circ} \mathrm{C}$ for $\left.2 \mathrm{~min}\right)$, then 9 cycles of $\left(95^{\circ} \mathrm{C}\right.$ for $35 \mathrm{~s}, 55^{\circ} \mathrm{C}$ for $55 \mathrm{~s}, 72^{\circ} \mathrm{C}$ for $\left.3 \mathrm{~min}\right)$, then $72^{\circ} \mathrm{C}$ for $10 \mathrm{~min}$ and then $4^{\circ} \mathrm{C}$ soak until the samples were removed.

For all PCR reactions a gel was run to ensure the DNA was replicated. $5 \mu \mathrm{L}$ of PCR product were added to each well on a 1.5\% agrose gel. The gel ran for 20 min at 100 Volts and $400 \mathrm{~mA}$. DNA was visualized with ethidium bromide. For PCR successful samples, the PCR product clean up was performed. Using the MoBio PCR Ultra-Clean kit, 5 volumes SpinBind solution were added to each well and pipeted up and down to mix. Sample replicates were then combined into a spin filter and centrifuged for $30 \mathrm{sec}$ at 10,000 x g. Permeate was discarded into MoBio waste container. Then $300 \mathrm{~mL}$ of SpinClean buffer was added to each spin filter and centrifuged for $30 \mathrm{sec}$ at $10,000 \mathrm{x} \mathrm{g}$. Permeate was discarded. Spin filters were centrifuged for $120 \mathrm{sec}$ at $10 \mathrm{x} \mathrm{kg}$ to remove 
any remaining fluid. Spin filters were transferred to clean $2.0-\mathrm{mL}$ collection tubes. $60 \mathrm{~mL}$ of PCR water was added to spin filter and incubated for $10 \mathrm{~min}$. Samples were centrifuged for $60 \mathrm{sec}$ at $10,000 \mathrm{x}$ g. Spin filters were discarded. DNA was quantified with the Spectradrop spectrometer. DNA was stored at $-20^{\circ} \mathrm{C}$.

\section{Sequencing and Data Analysis}

Approximately $50 \mathrm{ng}$ of each DNA sample was shipped overnight to Sequatech in Mountain View, Ca. Primers were also mailed with the samples. For bacteria, the 46F, $530 \mathrm{R}, 530 \mathrm{~F}$ and $1492 \mathrm{R}$ primers were used. For fungi the $8 \mathrm{dF}$ and $1525 \mathrm{R}$ primers were used. Sequence results were emailed back in 2 days. The sequences were then aligned using SeqMan software. Sequences were analyzed using the National Center for Biotechnology Information (NCBI) database known as BLAST (Basic Local Alignment Search Tool). The most likely identity of the organism as well as the query cover, indent, and E value were recorded. Query cover indicates the percent of the query sequence that overlaps with the subject sequence. The indent specifies the percent of the subject sequence that overlaps at the beginning of the sequence. It indicates how much sequence could have been lost due to where the primer is located on the gene. E value is a measure of random background noise. It describes the hits one can expect to see by chance when searching a database of a specific size. The closer the $\mathrm{E}$ value is to 0 the more significant the match is. 
5.0 Results and Discussion

\subsection{Soil Sample Locations and Contaminant Concentrations}

The 30 soil sampling sites used for this research are shown on a map in Figure 5.1.

Chemical data for these sample sites is presented in Table 5.1. This table shows the concentrations of each COI at the 30 sample sites, as well as the total metals concentrations. Sample name, location code, and date sampled are also provided. Constituents marked "unknown" were not measured at that location. 


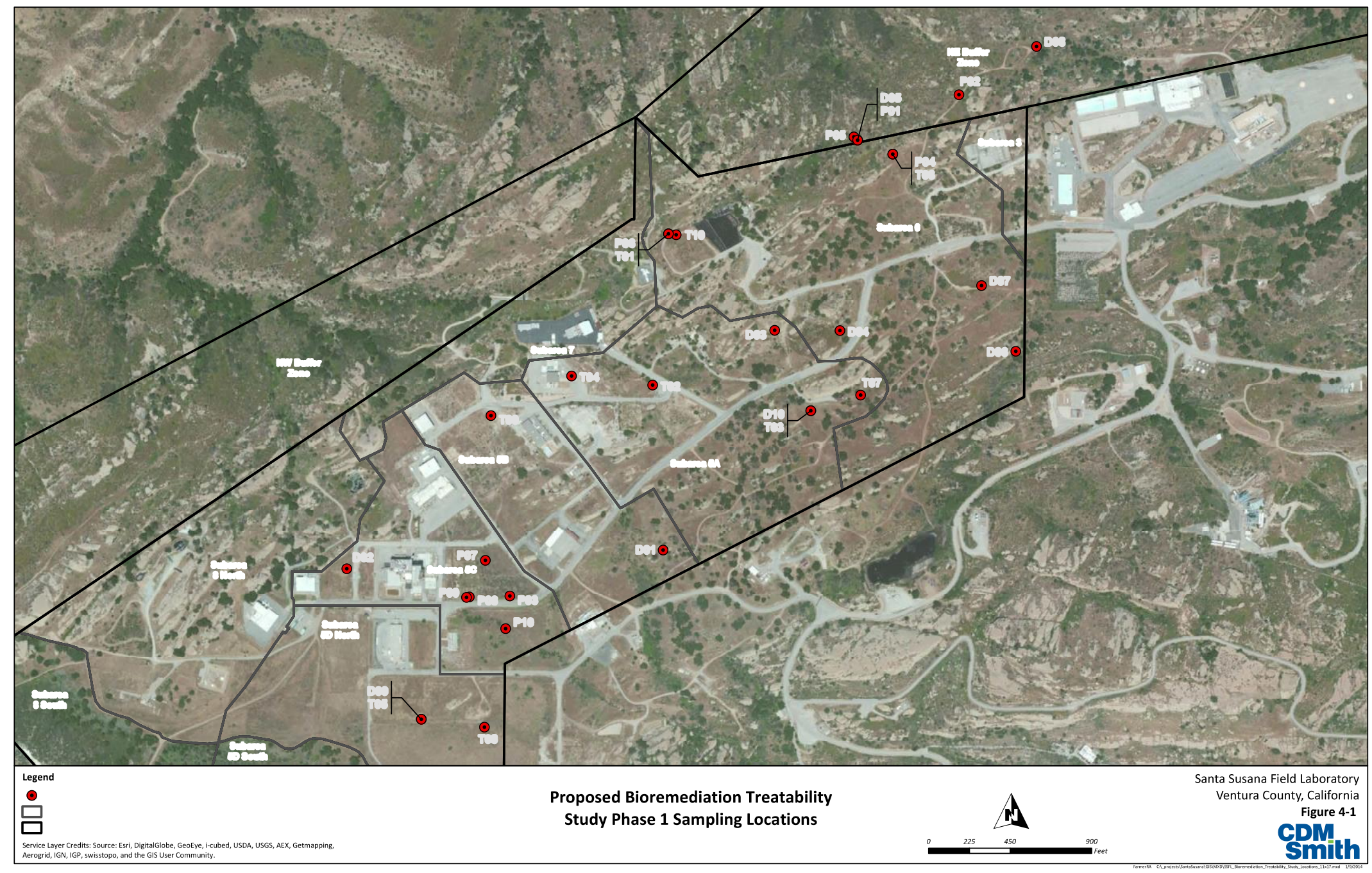

Figure 5.1: Soil Sample Sites on SSFL (CDM Smith 2014) 
Table 5.1: Chemical Data of the 30 Selected Sample Sites

\begin{tabular}{|c|c|c|c|c|c|c|c|}
\hline $\begin{array}{l}\text { TPH/ } \\
\text { PAH }\end{array}$ & $\begin{array}{l}\text { Location } \\
\text { Code }\end{array}$ & $\begin{array}{c}\text { Date } \\
\text { Sampled }\end{array}$ & $\begin{array}{c}\mathrm{TPH} \\
(\mathrm{mg} / \mathrm{kg})\end{array}$ & $\begin{array}{l}\text { PAHs } \\
(\mathrm{ug} / \mathrm{kg})\end{array}$ & $\begin{array}{c}\text { PCB } \\
(\mathrm{ug} / \mathrm{kg})\end{array}$ & $\begin{array}{l}\text { Dioxins } \\
(\mathrm{ng} / \mathrm{kg})\end{array}$ & $\begin{array}{c}\text { Total } \\
\text { Metals } \\
(\mathrm{mg} / \mathrm{kg})\end{array}$ \\
\hline T01 & $\begin{array}{c}\text { SL-291- } \\
\text { SA6 }\end{array}$ & $9 / 1 / 11$ & 1020 & 8390 & 473 & 4560 & 40700 \\
\hline T02 & $\begin{array}{l}\text { SL-012- } \\
\text { SA5A }\end{array}$ & $3 / 7 / 11$ & 2410 & 3280 & 8.28 & 3360 & 38100 \\
\hline T03 & $\begin{array}{c}\text { SL-058- } \\
\text { SA5A }\end{array}$ & 4/14/11 & 121 & 6020 & 15.8 & 5150 & 97800 \\
\hline T04 & $\begin{array}{c}\text { SL-118- } \\
\text { SA5A }\end{array}$ & $3 / 14 / 11$ & 1850 & 2820 & 7.34 & 3160 & 39000 \\
\hline T05 & $\begin{array}{l}\text { SL-116- } \\
\text { SA5DN }\end{array}$ & $6 / 28 / 11$ & 119 & 5630 & 15.1 & 5450 & 108000 \\
\hline T06 & $\begin{array}{c}\text { SL-250- } \\
\text { SA6 }\end{array}$ & 9/9/11 & 1190 & 2780 & 635 & 4100 & 41200 \\
\hline T07 & $\begin{array}{c}\text { SL-064- } \\
\text { SA5A }\end{array}$ & $4 / 22 / 11$ & 119 & 5817 & 15.4 & 4860 & 118000 \\
\hline T08 & $\begin{array}{l}\text { SL-144- } \\
\text { SA5DN }\end{array}$ & $5 / 25 / 11$ & 119 & 5820 & 15.3 & 4720 & 136000 \\
\hline T09 & $\begin{array}{c}\text { SL-063- } \\
\text { SA5B }\end{array}$ & $1 / 11 / 11$ & 673 & 37.1 & 7.10 & 28.4 & 57800 \\
\hline $\mathrm{T} 10$ & $\begin{array}{c}\text { SL-104- } \\
\text { SA6 }\end{array}$ & $8 / 7 / 11$ & 538 & 3390 & 17.8 & 3140 & 45700 \\
\hline \multicolumn{8}{|l|}{ PCB } \\
\hline P01 & $\begin{array}{c}\text { SL-040- } \\
\text { NBZ }\end{array}$ & $3 / 28 / 12$ & 367 & 775 & 32000 & 6570 & 69400 \\
\hline $\mathrm{P} 02$ & $\begin{array}{c}\text { SL-062- } \\
\text { NBZ }\end{array}$ & $3 / 21 / 12$ & 23.2 & 347 & 829 & 3010 & 38400 \\
\hline $\mathrm{P} 03$ & $\begin{array}{c}\text { 5C_DG- } \\
556 \mathrm{D}\end{array}$ & $5 / 15 / 12$ & unknown & unknown & 645 & unknown & unknown \\
\hline P04 & $\begin{array}{c}\text { SL-250- } \\
\text { SA6 }\end{array}$ & $9 / 9 / 11$ & 1190 & 2780 & 635 & 4100 & 41200 \\
\hline P05 & $\begin{array}{c}\text { SL-039- } \\
\text { NBZ }\end{array}$ & $3 / 28 / 12$ & 232 & 403 & 576 & 2930 & 38500 \\
\hline P06 & $\begin{array}{l}\text { SL-291- } \\
\text { SA6 }\end{array}$ & $9 / 1 / 11$ & 1020 & 8390 & 473 & 4560 & 40700 \\
\hline P07 & $\begin{array}{c}\text { 5C_DG- } \\
644\end{array}$ & $5 / 8 / 12$ & unknown & unknown & 387 & unknown & unknown \\
\hline P08 & $\begin{array}{c}\text { 5C_DG- } \\
558 \mathrm{~B}\end{array}$ & $6 / 1 / 12$ & unknown & unknown & 360 & unknown & unknown \\
\hline P09 & $\begin{array}{c}\text { 5C_DG- } \\
558 \mathrm{C}\end{array}$ & $6 / 1 / 12$ & unknown & unknown & 360 & unknown & unknown \\
\hline P10 & $\begin{array}{c}\text { 5C_DG- } \\
634\end{array}$ & $4 / 20 / 12$ & unknown & unknown & 360 & unknown & unknown \\
\hline
\end{tabular}




\begin{tabular}{|c|c|c|c|c|c|c|c|}
\hline Dioxins & $\begin{array}{c}\text { Location } \\
\text { Code }\end{array}$ & $\begin{array}{c}\text { Date } \\
\text { Sampled }\end{array}$ & $\begin{array}{c}\text { TPH } \\
(\mathrm{mg} / \mathrm{kg})\end{array}$ & $\begin{array}{c}\text { PAHs } \\
(\mathrm{ug} / \mathrm{kg})\end{array}$ & $\begin{array}{c}\text { PCB } \\
(\mathrm{ug} / \mathrm{kg})\end{array}$ & $\begin{array}{c}\text { Dioxins } \\
(\mathrm{ng} / \mathrm{kg})\end{array}$ & $\begin{array}{c}\text { Total } \\
\text { Metals } \\
(\mathrm{mg} / \mathrm{kg})\end{array}$ \\
\hline D01 & $\begin{array}{c}\text { SL-273- } \\
\text { SA5B }\end{array}$ & $12 / 17 / 10$ & unknown & 53.9 & 124 & 17200 & 71000 \\
\hline D02 & $\begin{array}{c}5 \text { C_DG- } \\
596\end{array}$ & $5 / 3 / 12$ & unknown & unknown & unknown & 20200 & unknown \\
\hline D03 & $\begin{array}{c}\text { SL-221- } \\
\text { SA5A }\end{array}$ & $3 / 29 / 11$ & 70.0 & 2850 & 327 & 13100 & 129000 \\
\hline D04 & $\begin{array}{c}\text { SL-321- } \\
\text { SA6 }\end{array}$ & $8 / 3 / 11$ & 22.2 & 2680 & 14.5 & 7640 & 54300 \\
\hline D05 & $\begin{array}{c}\text { SL-040- } \\
\text { NBZ }\end{array}$ & $3 / 28 / 12$ & 366 & 775 & 32000 & 6570 & 69400 \\
\hline D06 & $\begin{array}{c}\text { SL-229- } \\
\text { SA6 }\end{array}$ & $10 / 21 / 11$ & unknown & 2970 & 25.6 & 6470 & 39200 \\
\hline D07 & $\begin{array}{c}\text { SL-224- } \\
\text { SA6 }\end{array}$ & $8 / 30 / 11$ & 20 & 5440 & 25.2 & 6110 & 134000 \\
\hline D08 & $\begin{array}{c}\text { SL-068- } \\
\text { NBZ }\end{array}$ & $3 / 16 / 12$ & 15.3 & 780 & 269 & 5680 & 81000 \\
\hline D09 & $\begin{array}{c}\text { SL-116- } \\
\text { SA5DN }\end{array}$ & $6 / 28 / 11$ & 119 & 5630 & 15.1 & 5450 & 108000 \\
\hline D10 & $\begin{array}{c}\text { SL-058- } \\
\text { SA5A }\end{array}$ & $2 / 21 / 11$ & 121 & 6020 & 15.8 & 5150 & 97800 \\
\hline
\end{tabular}

\subsection{Cultured and Identified Microbes}

The conditions of the culturing experiments and the resulting cultures isolated are summarized in Tables 5.2 for bacteria and Table 5.3 for fungi. From these 336 separate culturing experiments (including replicates), 45 colonies were isolated from the soil samples from SSFL. Of these 45, 9 were fungi that were isolated on solid, Bushnell Haas media that was spiked with a COI. The remaining 36 were bacterial colonies, 20 were isolated on solid, Bushnell Haas media that was spiked with a COI and 16 were isolated from TSB cultures spiked with COIs. See Figure 5.2 for examples of solid Bushnell Haas plates with colonies. Table 5.4 summarizes how many colonies and if the colonies were bacteria or fungi for each COI. 
Table 5.2: Bacterial Culturing Conditions and Results

\begin{tabular}{|c|c|c|c|c|c|c|c|c|c|c|c|c|c|c|c|}
\hline $\begin{array}{l}E \\
x \\
p\end{array}$ & $\begin{array}{l}\mathrm{S} \\
\mathrm{t} \\
\mathrm{e} \\
\mathrm{p} \\
\#\end{array}$ & Medium & $\begin{array}{l}\text { Diesel } \\
\text { Fuel } \\
\text { Conc } \\
\text { (ppm) }\end{array}$ & $\begin{array}{l}\text { Potential } \\
\text { TPH } \\
\text { Degraders } \\
\text { Isolated }\end{array}$ & $\begin{array}{l}\text { Cells/g } \\
\text { based } \\
\text { on } \\
\text { Plate } \\
\text { Counts }\end{array}$ & $\begin{array}{l}\text { Naphth } \\
\text {-alene } \\
\text { Conc } \\
\text { (ppm) }\end{array}$ & $\begin{array}{l}\text { Potential } \\
\text { PAH } \\
\text { Degraders } \\
\text { Isolated }\end{array}$ & $\begin{array}{l}\text { Cells/g } \\
\text { based } \\
\text { on } \\
\text { Plate } \\
\text { Counts }\end{array}$ & $\begin{array}{l}\text { PCB } \\
1 \\
\text { Conc } \\
(\mathrm{ppb})\end{array}$ & $\begin{array}{l}\text { Potential } \\
\text { PCB } \\
\text { Degraders } \\
\text { Isolated }\end{array}$ & $\begin{array}{l}\text { Cells/g } \\
\text { based } \\
\text { on } \\
\text { Plate } \\
\text { Counts }\end{array}$ & $\begin{array}{l}\text { DBZ } \\
\text { Conc } \\
(\mathrm{ppb})\end{array}$ & $\begin{array}{l}\text { Potential } \\
\text { Dioxin } \\
\text { Degraders } \\
\text { Isolated }\end{array}$ & $\begin{array}{l}\text { Cells/g } \\
\text { based } \\
\text { on } \\
\text { Plate } \\
\text { Counts }\end{array}$ & $\begin{array}{l}\text { Total } \\
\text { Incuba } \\
\text {-tion } \\
\text { Time }\end{array}$ \\
\hline 1 & 1 & $\begin{array}{c}\text { CFMM } \\
\text { Plate + } \\
\text { MCs }\end{array}$ & 100 & $\mathrm{x}$ & 400 & 50 & $x$ & 0 & 10 & $\mathrm{x}$ & 400 & 10 & $x$ & 0 & $\begin{array}{c}5 \\
\text { weeks }\end{array}$ \\
\hline 1 & 2 & TSB & 0 & 1 & $\mathrm{x}$ & 0 & 0 & $x$ & 0 & 1 & $\mathrm{x}$ & 0 & 0 & $x$ & 1 week \\
\hline 2 & 1 & $\begin{array}{c}\text { CFMM } \\
\text { Plate + } \\
\text { MCs }\end{array}$ & 1000 & $\mathrm{x}$ & 600 & 500 & $\mathrm{x}$ & 1200 & 100 & $\mathrm{x}$ & 700 & 100 & $\mathrm{x}$ & 500 & $\begin{array}{c}5 \\
\text { weeks }\end{array}$ \\
\hline 2 & 2 & TSB & 0 & 2 & $\mathrm{x}$ & 0 & 3 & $\mathrm{x}$ & 0 & 3 & $\mathrm{x}$ & 0 & 1 & $\mathrm{x}$ & 1 week \\
\hline 3 & 1 & $\begin{array}{c}\text { CFMM } \\
\text { Plate + } \\
\text { MCs }\end{array}$ & 10000 & $\mathrm{x}$ & 200 & 5000 & $\mathrm{x}$ & 600 & 1000 & $\mathrm{x}$ & 500 & 1000 & $\mathrm{x}$ & 700 & $\begin{array}{c}5 \\
\text { weeks }\end{array}$ \\
\hline 3 & 2 & TSB & 0 & 2 & $\mathrm{x}$ & 0 & 2 & $\mathrm{x}$ & 0 & 2 & $\mathrm{x}$ & 0 & 2 & $\mathrm{x}$ & 1 week \\
\hline 4 & 1 & $\begin{array}{c}\text { CFMM } \\
\text { Plate + } \\
\text { MCs } \\
\end{array}$ & $\mathrm{x}$ & $\mathrm{x}$ & $\mathrm{x}$ & $\mathrm{x}$ & $\mathrm{x}$ & $\mathrm{x}$ & $\begin{array}{l}100, \\
000\end{array}$ & $\mathbf{x}$ & 0 & $\begin{array}{l}100, \\
000\end{array}$ & $\mathbf{x}$ & 0 & $\begin{array}{c}5 \\
\text { weeks }\end{array}$ \\
\hline 4 & 2 & TSB & $\mathrm{x}$ & $\mathrm{x}$ & $\mathrm{x}$ & $\mathrm{x}$ & $\mathrm{x}$ & $\mathrm{x}$ & $\mathbf{0}$ & $\mathbf{0}$ & $\mathbf{x}$ & $\mathbf{0}$ & $\mathbf{0}$ & $\mathbf{x}$ & 1 week \\
\hline 5 & 1 & $\begin{array}{c}\text { CFMM } \\
\text { Plate + } \\
\text { MCs }\end{array}$ & 50 & $\mathrm{x}$ & 200 & 10 & $x$ & 0 & $\mathrm{x}$ & $\mathrm{x}$ & $\mathrm{x}$ & $\mathrm{x}$ & $\mathrm{x}$ & $\mathrm{x}$ & $\begin{array}{c}5 \\
\text { weeks }\end{array}$ \\
\hline 5 & 2 & TSB & 0 & 1 & $\mathrm{x}$ & 0 & 0 & $x$ & $\mathrm{x}$ & $\mathrm{x}$ & $\mathrm{x}$ & $\mathrm{x}$ & $\mathrm{x}$ & $\mathrm{x}$ & 1 week \\
\hline
\end{tabular}




\begin{tabular}{|c|c|c|c|c|c|c|c|c|c|c|c|c|c|c|c|}
\hline $\begin{array}{l}E \\
x \\
p\end{array}$ & $\begin{array}{l}\mathrm{S} \\
\mathrm{t} \\
\mathrm{e} \\
\mathrm{p} \\
\#\end{array}$ & Medium & $\begin{array}{l}\text { Diese } \\
1 \text { Fuel } \\
\text { Conc } \\
(\mathrm{ppm})\end{array}$ & $\begin{array}{l}\text { Potential } \\
\text { TPH } \\
\text { Degraders } \\
\text { Isolated }\end{array}$ & $\begin{array}{l}\text { Cells/g } \\
\text { based } \\
\text { on } \\
\text { Plate } \\
\text { Counts }\end{array}$ & $\begin{array}{l}\text { Naphth } \\
\text {-alene } \\
\text { Conc } \\
\text { (ppm) }\end{array}$ & $\begin{array}{l}\text { Potential } \\
\text { PAH } \\
\text { Degraders } \\
\text { Isolated }\end{array}$ & $\begin{array}{l}\text { Cells/g } \\
\text { based } \\
\text { on } \\
\text { Plate } \\
\text { Counts }\end{array}$ & $\begin{array}{l}\text { PCB } \\
1 \\
\text { Conc } \\
(\mathrm{ppb})\end{array}$ & $\begin{array}{l}\text { Potential } \\
\text { PCB } \\
\text { Degraders } \\
\text { Isolated }\end{array}$ & $\begin{array}{l}\text { Cells/g } \\
\text { based } \\
\text { on } \\
\text { Plate } \\
\text { Counts }\end{array}$ & $\begin{array}{l}\text { DBZ } \\
\text { Conc } \\
(\mathrm{ppb})\end{array}$ & $\begin{array}{l}\text { Potential } \\
\text { Dioxin } \\
\text { Degraders } \\
\text { Isolated }\end{array}$ & $\begin{array}{l}\text { Cells/g } \\
\text { based } \\
\text { on } \\
\text { Plate } \\
\text { Counts }\end{array}$ & $\begin{array}{l}\text { Total } \\
\text { Incuba } \\
\text {-tion } \\
\text { Time }\end{array}$ \\
\hline 6 & 1 & $\begin{array}{c}\text { CFMM } \\
\text { Liquid + } \\
\text { MCs } \\
\end{array}$ & 100 & $x$ & $x$ & 50 & $x$ & $x$ & 10 & $x$ & $x$ & 10 & $x$ & $x$ & $\begin{array}{c}5 \\
\text { weeks }\end{array}$ \\
\hline 6 & 2 & $\begin{array}{c}\text { CFMM } \\
\text { Plates + } \\
\text { MCs } \\
\end{array}$ & 100 & 0 & $x$ & 50 & 0 & $x$ & 10 & 0 & $x$ & 10 & 0 & $x$ & $\begin{array}{c}5 \\
\text { weeks }\end{array}$ \\
\hline 6 & 3 & TSB & 0 & $x$ & $x$ & 0 & $x$ & $x$ & 0 & $x$ & $x$ & 0 & $x$ & $x$ & 1 week \\
\hline 7 & 1 & $\begin{array}{c}\text { CFMM } \\
\text { Liquid + } \\
\text { MCs }\end{array}$ & 1000 & $x$ & $x$ & 500 & $x$ & $x$ & 100 & $x$ & $x$ & 100 & $x$ & $x$ & $\begin{array}{c}5 \\
\text { weeks }\end{array}$ \\
\hline 7 & 2 & $\begin{array}{c}\text { CFMM } \\
\text { Plates + } \\
\text { MCs }\end{array}$ & 1000 & 0 & $x$ & 500 & 0 & $x$ & 100 & 0 & $x$ & 100 & 0 & $x$ & $\begin{array}{c}5 \\
\text { weeks }\end{array}$ \\
\hline 7 & 3 & TSB & 0 & $x$ & $x$ & 0 & $x$ & $x$ & 0 & $x$ & $x$ & 0 & $x$ & $x$ & 1 week \\
\hline 8 & 1 & $\begin{array}{c}\text { CFMM } \\
\text { Liquid + } \\
\text { MCs }\end{array}$ & 10000 & $x$ & $x$ & 5000 & $x$ & $x$ & 1000 & $x$ & $x$ & 1000 & $x$ & $x$ & $\begin{array}{c}5 \\
\text { weeks }\end{array}$ \\
\hline 8 & 2 & $\begin{array}{c}\text { CFMM } \\
\text { Plates + } \\
\text { MCs } \\
\end{array}$ & 10000 & 0 & $x$ & 5000 & 0 & $x$ & 1000 & 0 & $x$ & 1000 & 0 & $x$ & $\begin{array}{c}5 \\
\text { weeks }\end{array}$ \\
\hline 8 & 3 & TSB & 0 & $x$ & $x$ & 0 & $x$ & $x$ & 0 & $x$ & $x$ & 0 & $x$ & $x$ & 1 week \\
\hline 9 & 1 & $\begin{array}{c}\text { CFMM } \\
\text { Liquid + } \\
\text { MCs }\end{array}$ & 100 & $x$ & $x$ & 50 & $x$ & $x$ & 10 & $x$ & $x$ & 10 & $x$ & $x$ & $\begin{array}{c}5 \\
\text { weeks }\end{array}$ \\
\hline 9 & 2 & $\begin{array}{c}\mathrm{TSA}+ \\
\mathrm{MCs}\end{array}$ & 100 & 0 & $x$ & 50 & 0 & $x$ & 10 & 0 & $x$ & 10 & 0 & $x$ & $\begin{array}{c}5 \\
\text { weeks }\end{array}$ \\
\hline 9 & 3 & TSB & 0 & $x$ & $x$ & 0 & $x$ & $x$ & 0 & $x$ & $x$ & 0 & $x$ & $x$ & 1 week \\
\hline
\end{tabular}




\begin{tabular}{|c|c|c|c|c|c|c|c|c|c|c|c|c|c|c|c|}
\hline $\begin{array}{l}E \\
x \\
p \\
\#\end{array}$ & \begin{tabular}{l|}
$\mathrm{S}$ \\
$\mathrm{t}$ \\
$\mathrm{e}$ \\
$\mathrm{p}$ \\
$\#$
\end{tabular} & Medium & $\begin{array}{l}\text { Diese } \\
\text { 1 Fuel } \\
\text { Conc } \\
\text { (ppm) }\end{array}$ & $\begin{array}{l}\text { Potential } \\
\text { TPH } \\
\text { Degrader } \\
\text { s Isolated }\end{array}$ & $\begin{array}{l}\text { Cells/g } \\
\text { based } \\
\text { on } \\
\text { Plate } \\
\text { Counts }\end{array}$ & $\begin{array}{l}\text { Napht } \\
\text { halen } \\
\text { e } \\
\text { Conc } \\
\text { (ppm) }\end{array}$ & $\begin{array}{l}\text { Potential } \\
\text { PAH } \\
\text { Degraders } \\
\text { Isolated }\end{array}$ & $\begin{array}{l}\text { Cells/g } \\
\text { based } \\
\text { on } \\
\text { Plate } \\
\text { Counts }\end{array}$ & $\begin{array}{l}\text { PCB } \\
1 \\
\text { Conc } \\
(\mathrm{ppb})\end{array}$ & $\begin{array}{l}\text { Potential } \\
\text { PCB } \\
\text { Degraders } \\
\text { Isolated }\end{array}$ & $\begin{array}{l}\text { Cells/g } \\
\text { based } \\
\text { on } \\
\text { Plate } \\
\text { Counts }\end{array}$ & $\begin{array}{l}\text { DBZ } \\
\text { Conc } \\
(\mathrm{ppb})\end{array}$ & $\begin{array}{l}\text { Potential } \\
\text { Dioxin } \\
\text { Degrader } \\
\text { s Isolated }\end{array}$ & $\begin{array}{l}\text { Cells/g } \\
\text { based } \\
\text { on } \\
\text { Plate } \\
\text { Counts }\end{array}$ & $\begin{array}{l}\text { Total } \\
\text { Incuba } \\
\text {-tion } \\
\text { Time }\end{array}$ \\
\hline $\begin{array}{l}1 \\
0\end{array}$ & 1 & $\begin{array}{l}\text { CF Liquid } \\
+\mathrm{MCs}\end{array}$ & 1000 & $x$ & $x$ & 500 & $x$ & $x$ & 100 & $x$ & $x$ & 100 & $x$ & $x$ & $\begin{array}{c}5 \\
\text { weeks }\end{array}$ \\
\hline $\begin{array}{l}1 \\
0\end{array}$ & 2 & $\begin{array}{c}\mathrm{TSA}+ \\
\mathrm{MCs}\end{array}$ & 1000 & 0 & $x$ & 500 & 0 & $x$ & 100 & 0 & $x$ & 100 & 0 & $x$ & $\begin{array}{c}5 \\
\text { weeks }\end{array}$ \\
\hline $\begin{array}{l}1 \\
0\end{array}$ & 3 & TSB & 0 & $x$ & $x$ & 0 & $x$ & $x$ & 0 & $x$ & $x$ & 0 & $x$ & $x$ & 1 week \\
\hline $\begin{array}{l}1 \\
1\end{array}$ & 1 & $\begin{array}{c}\text { CFMM } \\
\text { Liquid + } \\
\text { MCs }\end{array}$ & 10000 & $x$ & $x$ & 5000 & $x$ & $x$ & 1000 & $x$ & $x$ & 1000 & $x$ & $x$ & $\begin{array}{c}5 \\
\text { weeks }\end{array}$ \\
\hline \begin{tabular}{l|}
1 \\
1 \\
\end{tabular} & 2 & $\begin{array}{c}\mathrm{TSA}+ \\
\mathrm{MCs}\end{array}$ & 10000 & 0 & $x$ & 5000 & 0 & $x$ & 1000 & 0 & $x$ & 1000 & 0 & $x$ & $\begin{array}{c}5 \\
\text { weeks } \\
\end{array}$ \\
\hline $\begin{array}{l}1 \\
1\end{array}$ & 3 & TSB & 0 & $x$ & $x$ & 0 & $x$ & $x$ & 0 & $x$ & $x$ & 0 & $x$ & $x$ & 1 week \\
\hline $\begin{array}{l}1 \\
2\end{array}$ & 1 & $\begin{array}{l}\text { TSB Liquid } \\
+\mathrm{MCs}\end{array}$ & 100 & $\mathrm{x}$ & $\mathrm{x}$ & 50 & $\mathrm{x}$ & $\mathrm{x}$ & 10 & $x$ & $x$ & 10 & $\mathrm{x}$ & $\mathrm{x}$ & $\begin{array}{c}5 \\
\text { weeks }\end{array}$ \\
\hline $\begin{array}{l}1 \\
2\end{array}$ & 2 & TSA & 100 & 2 & $\mathrm{x}$ & 50 & 1 & $\mathrm{x}$ & 10 & 0 & $x$ & 10 & 4 & $\mathrm{x}$ & $\begin{array}{c}5 \\
\text { weeks }\end{array}$ \\
\hline $\begin{array}{l}1 \\
2\end{array}$ & 3 & TSB & 0 & $\mathrm{x}$ & $\mathrm{x}$ & 0 & $\mathrm{x}$ & $\mathrm{x}$ & 0 & $x$ & $x$ & 0 & $\mathrm{x}$ & $\mathrm{x}$ & 1 week \\
\hline $\begin{array}{l}1 \\
3\end{array}$ & 1 & $\begin{array}{l}\text { TSB Liquid } \\
+\mathrm{MCs}\end{array}$ & 1000 & $\mathrm{x}$ & $\mathrm{x}$ & 500 & $\mathrm{x}$ & $\mathrm{x}$ & 100 & $\mathrm{x}$ & $\mathrm{x}$ & 100 & $\mathrm{x}$ & $\mathrm{x}$ & $\begin{array}{c}5 \\
\text { weeks }\end{array}$ \\
\hline $\begin{array}{l}1 \\
3\end{array}$ & 2 & TSA & 1000 & 2 & $\mathrm{x}$ & 500 & 2 & $\mathrm{x}$ & 100 & 2 & $\mathrm{x}$ & 100 & 2 & $\mathrm{x}$ & $\begin{array}{c}5 \\
\text { weeks }\end{array}$ \\
\hline $\begin{array}{l}1 \\
3\end{array}$ & 3 & TSB & 0 & $\mathrm{x}$ & $\mathrm{x}$ & 0 & $\mathrm{x}$ & $\mathrm{x}$ & 0 & $\mathrm{x}$ & $\mathrm{x}$ & 0 & $\mathrm{x}$ & $\mathrm{x}$ & 1 week \\
\hline \begin{tabular}{l|}
1 \\
4
\end{tabular} & 1 & $\begin{array}{l}\text { TSB Liquid } \\
+\mathrm{MCs}\end{array}$ & 10000 & $\mathbf{x}$ & $\mathbf{x}$ & 5000 & $\mathbf{x}$ & $\mathbf{x}$ & 1000 & $\mathrm{x}$ & $\mathrm{x}$ & 1000 & $\mathbf{x}$ & $\mathbf{x}$ & $\begin{array}{c}5 \\
\text { weeks }\end{array}$ \\
\hline $\begin{array}{l}1 \\
4\end{array}$ & 2 & TSA & 10000 & 0 & $\mathbf{x}$ & 5000 & 0 & $\mathbf{x}$ & 1000 & 1 & $\mathrm{x}$ & 1000 & 0 & $\mathbf{x}$ & $\begin{array}{c}5 \\
\text { weeks }\end{array}$ \\
\hline $\begin{array}{l}1 \\
4\end{array}$ & 3 & TSB & 0 & $\mathbf{x}$ & $\mathbf{x}$ & 0 & $\mathbf{x}$ & $\mathbf{x}$ & 0 & $\mathrm{x}$ & $\mathrm{x}$ & 0 & $\mathbf{x}$ & $\mathbf{x}$ & 1 week \\
\hline
\end{tabular}


Table 5.2 Notes:

MCs: Model Chemicals, CFMM or CF: Carbon Free Mineral Media, TSA: Tryptone Soy Agar, TSB: Tryptone Soy Broth.

Step indicates the sequence of transfers from media to media within a single culturing experiment.

$\mathrm{X}$ indicates that there is no data. This is caused by the cell either being not applicable or not attempted (in the case of the gray cells).

Bold text indicates experiment portions that yielded no growth of organisms or control organisms, indicating possible toxic effects of concentration. Italic text indicate experiment portions that yielded no growth of organisms or control organisms, indicating a possible stunting of growth from low available carbon. 
Table 5.3: Fungal Culturing Conditions and Results

\begin{tabular}{|c|c|c|c|c|c|c|c|c|c|c|c|c|c|c|c|}
\hline $\begin{array}{l}E \\
x \\
p\end{array}$ & $\begin{array}{l}\mathrm{S} \\
\mathrm{t} \\
\mathrm{e} \\
\mathrm{p} \\
\#\end{array}$ & Medium & $\begin{array}{l}\text { Diesel } \\
\text { Fuel } \\
\text { Conc } \\
(\mathrm{ppm})\end{array}$ & $\begin{array}{l}\text { Potential } \\
\text { TPH } \\
\text { Degraders } \\
\text { Isolated }\end{array}$ & $\begin{array}{l}\text { Cells/g } \\
\text { based } \\
\text { on Plate } \\
\text { Counts }\end{array}$ & $\begin{array}{l}\text { Naphth } \\
\text {-alene } \\
\text { Conc } \\
\text { (ppm) }\end{array}$ & $\begin{array}{l}\text { Potential } \\
\text { PAH } \\
\text { Degraders } \\
\text { Isolated }\end{array}$ & $\begin{array}{l}\text { Cells/g } \\
\text { based } \\
\text { on } \\
\text { Plate } \\
\text { Counts }\end{array}$ & $\begin{array}{l}\text { PCB } \\
1 \\
\text { Conc } \\
\text { (ppb) }\end{array}$ & $\begin{array}{l}\text { Potentia } \\
1 \text { PCB } \\
\text { Degrade } \\
\text { rs } \\
\text { Isolated }\end{array}$ & $\begin{array}{l}\text { Cells/g } \\
\text { based } \\
\text { on } \\
\text { Plate } \\
\text { Counts }\end{array}$ & $\begin{array}{l}\text { DBZ } \\
\text { Conc } \\
(p p b)\end{array}$ & $\begin{array}{l}\text { Potential } \\
\text { Dioxin } \\
\text { Degrader } \\
\text { s Isolated }\end{array}$ & $\begin{array}{l}\text { Cells/g } \\
\text { based } \\
\text { on } \\
\text { Plate } \\
\text { Counts }\end{array}$ & $\begin{array}{l}\text { Total } \\
\text { Incuba } \\
\text {-tion } \\
\text { Time }\end{array}$ \\
\hline 1 & 1 & $\begin{array}{c}\text { CFMM } \\
\text { Plate + } \\
\text { MCs }\end{array}$ & 100 & $x$ & 0 & 50 & $x$ & 0 & 10 & $x$ & 0 & 10 & $x$ & 0 & $\begin{array}{c}5 \\
\text { weeks }\end{array}$ \\
\hline 1 & 2 & $\begin{array}{c}\text { YM } \\
\text { Liquid }\end{array}$ & 0 & 0 & $x$ & 0 & 0 & $x$ & 0 & 0 & $x$ & 0 & 0 & $x$ & 1 week \\
\hline 2 & 1 & $\begin{array}{c}\text { CFMM } \\
\text { Plate + } \\
\text { MCs }\end{array}$ & 1000 & $\mathrm{x}$ & 800 & 500 & $\mathrm{x}$ & 1100 & 100 & $x$ & 0 & 100 & $x$ & 0 & $\begin{array}{c}5 \\
\text { weeks }\end{array}$ \\
\hline 2 & 2 & $\begin{array}{c}\text { YM } \\
\text { Liquid }\end{array}$ & 0 & 1 & $\mathrm{x}$ & 0 & 1 & $\mathrm{x}$ & 0 & 0 & $x$ & 0 & 0 & $x$ & 1 week \\
\hline 3 & 1 & $\begin{array}{c}\text { CFMM } \\
\text { Plate + } \\
\text { MCs }\end{array}$ & 10000 & $\mathrm{x}$ & 1200 & 5000 & $\mathrm{x}$ & 700 & 1000 & $x$ & 0 & 1000 & $\mathrm{x}$ & 600 & $\begin{array}{c}5 \\
\text { weeks }\end{array}$ \\
\hline 3 & 2 & TSB & 0 & 1 & $\mathrm{x}$ & 0 & 1 & $\mathrm{x}$ & 0 & 0 & $x$ & 0 & 1 & $\mathrm{x}$ & 1 week \\
\hline 4 & 1 & $\begin{array}{c}\text { CFMM } \\
\text { Plate + } \\
\text { MCs }\end{array}$ & $\mathrm{x}$ & $\mathrm{x}$ & $\mathrm{x}$ & $\mathrm{x}$ & $\mathrm{x}$ & $\mathrm{x}$ & $\begin{array}{c}10000 \\
0\end{array}$ & $\mathrm{x}$ & 1400 & $\begin{array}{c}10000 \\
0\end{array}$ & $\mathrm{x}$ & 1200 & $\begin{array}{c}5 \\
\text { weeks }\end{array}$ \\
\hline 4 & 2 & $\begin{array}{c}\text { YM } \\
\text { Liquid }\end{array}$ & $\mathrm{x}$ & $\mathrm{x}$ & $\mathrm{x}$ & $\mathrm{x}$ & $\mathrm{x}$ & $\mathrm{x}$ & 0 & 2 & $\mathrm{x}$ & 0 & 2 & $\mathrm{x}$ & 1 week \\
\hline 5 & 1 & $\begin{array}{c}\text { CFMM } \\
\text { Plate + } \\
\text { MCs }\end{array}$ & 50 & $x$ & 0 & 10 & $x$ & 0 & $\mathrm{x}$ & $\mathrm{x}$ & $\mathrm{x}$ & $\mathrm{x}$ & $\mathrm{x}$ & $\mathrm{x}$ & $\begin{array}{c}5 \\
\text { weeks }\end{array}$ \\
\hline 5 & 2 & TSB & 0 & 0 & $x$ & 0 & 0 & $x$ & $\mathrm{x}$ & $\mathrm{x}$ & $\mathrm{x}$ & $\mathrm{x}$ & $\mathrm{x}$ & $\mathrm{x}$ & 1 week \\
\hline
\end{tabular}




\begin{tabular}{|c|c|c|c|c|c|c|c|c|c|c|c|c|c|c|c|}
\hline $\begin{array}{l}\mathrm{E} \\
\mathrm{x} \\
\mathrm{p} \\
\#\end{array}$ & $\begin{array}{l}\mathrm{S} \\
\mathrm{t} \\
\mathrm{e} \\
\mathrm{p} \\
\#\end{array}$ & Medium & $\begin{array}{l}\text { Diesel } \\
\text { Fuel } \\
\text { Conc } \\
\text { (ppm) }\end{array}$ & $\begin{array}{l}\text { Potential } \\
\text { TPH } \\
\text { Degraders } \\
\text { Isolated }\end{array}$ & $\begin{array}{l}\text { Cells/g } \\
\text { based } \\
\text { on } \\
\text { Plate } \\
\text { Counts }\end{array}$ & $\begin{array}{l}\text { Naph- } \\
\text { thalene } \\
\text { Conc } \\
\text { (ppm) }\end{array}$ & $\begin{array}{l}\text { Potential } \\
\text { PAH } \\
\text { Degrader } \\
\text { s Isolated }\end{array}$ & $\begin{array}{l}\text { Cells/g } \\
\text { based } \\
\text { on } \\
\text { Plate } \\
\text { Counts }\end{array}$ & $\begin{array}{l}\text { PCB } \\
1 \\
\text { Conc } \\
\text { (ppb) }\end{array}$ & $\begin{array}{l}\text { Potential } \\
\text { PCB } \\
\text { Degraders } \\
\text { Isolated }\end{array}$ & $\begin{array}{l}\text { Cells/g } \\
\text { based } \\
\text { on } \\
\text { Plate } \\
\text { Counts }\end{array}$ & $\begin{array}{l}\text { DBZ } \\
\text { Conc } \\
(p p b)\end{array}$ & $\begin{array}{l}\text { Potential } \\
\text { Dioxin } \\
\text { Degraders } \\
\text { Isolated }\end{array}$ & $\begin{array}{l}\text { Cells/g } \\
\text { based } \\
\text { on } \\
\text { Plate } \\
\text { Counts }\end{array}$ & $\begin{array}{l}\text { Total } \\
\text { Incub } \\
\text {-ation } \\
\text { Time }\end{array}$ \\
\hline 6 & 1 & $\begin{array}{c}\text { CFMM } \\
\text { Liquid + } \\
\text { MCs }\end{array}$ & 100 & $x$ & $x$ & 50 & $x$ & $x$ & 10 & $x$ & $x$ & 10 & $x$ & $x$ & $\begin{array}{c}5 \\
\text { weeks }\end{array}$ \\
\hline 6 & 2 & $\begin{array}{c}\text { CFMM } \\
\text { Plates + } \\
\text { MCs }\end{array}$ & 100 & 0 & $x$ & 50 & 0 & $x$ & 10 & 0 & $x$ & 10 & 0 & $x$ & $\begin{array}{c}5 \\
\text { weeks }\end{array}$ \\
\hline 6 & 3 & $\begin{array}{c}\text { YM } \\
\text { Liquid }\end{array}$ & 0 & $x$ & $x$ & 0 & $x$ & $x$ & 0 & $x$ & $x$ & 0 & $x$ & $x$ & $\begin{array}{c}1 \\
\text { week }\end{array}$ \\
\hline 7 & 1 & $\begin{array}{c}\text { CFMM } \\
\text { Liquid + } \\
\text { MCs }\end{array}$ & 1000 & $x$ & $x$ & 500 & $x$ & $x$ & 100 & $x$ & $x$ & 100 & $x$ & $x$ & $\begin{array}{c}5 \\
\text { weeks }\end{array}$ \\
\hline 7 & 2 & $\begin{array}{c}\text { CFMM } \\
\text { Plates + } \\
\text { MCs }\end{array}$ & 1000 & 0 & $x$ & 500 & 0 & $x$ & 100 & 0 & $x$ & 100 & 0 & $x$ & $\begin{array}{c}5 \\
\text { weeks }\end{array}$ \\
\hline 7 & 3 & $\begin{array}{c}\text { YM } \\
\text { Liquid }\end{array}$ & 0 & $x$ & $x$ & 0 & $x$ & $x$ & 0 & $x$ & $x$ & 0 & $x$ & $x$ & $\begin{array}{c}1 \\
\text { week }\end{array}$ \\
\hline 8 & 1 & $\begin{array}{c}\text { CFMM } \\
\text { Liquid + } \\
\text { MCs }\end{array}$ & 10000 & $x$ & $x$ & 5000 & $x$ & $x$ & 1000 & $x$ & $x$ & 1000 & $x$ & $x$ & $\begin{array}{c}5 \\
\text { weeks }\end{array}$ \\
\hline 8 & 2 & $\begin{array}{c}\text { CFMM } \\
\text { Plates+ } \\
\text { MCs }\end{array}$ & 10000 & 0 & $x$ & 5000 & 0 & $x$ & 1000 & 0 & $x$ & 1000 & 0 & $x$ & $\begin{array}{c}5 \\
\text { weeks }\end{array}$ \\
\hline 8 & 3 & $\begin{array}{c}\text { YM } \\
\text { Liquid }\end{array}$ & 0 & $x$ & $x$ & 0 & $x$ & $x$ & 0 & $x$ & $x$ & 0 & $x$ & $x$ & $\begin{array}{c}1 \\
\text { week }\end{array}$ \\
\hline 9 & 1 & $\begin{array}{c}\text { CFMM } \\
\text { Liquid + } \\
\text { MCs }\end{array}$ & 100 & $x$ & $x$ & 50 & $x$ & $x$ & 10 & $x$ & $x$ & 10 & $x$ & $x$ & $\begin{array}{c}5 \\
\text { weeks }\end{array}$ \\
\hline 9 & 2 & $\begin{array}{l}\mathrm{YM}+ \\
\mathrm{MCs}\end{array}$ & 100 & 0 & $x$ & 50 & 0 & $x$ & 10 & 0 & $x$ & 10 & 0 & $x$ & $\begin{array}{c}5 \\
\text { weeks }\end{array}$ \\
\hline 9 & 3 & $\begin{array}{c}\text { YM } \\
\text { Liquid }\end{array}$ & 0 & $x$ & $x$ & 0 & $x$ & $x$ & 0 & $x$ & $x$ & 0 & $x$ & $x$ & $\begin{array}{c}1 \\
\text { week }\end{array}$ \\
\hline
\end{tabular}




\begin{tabular}{|c|c|c|c|c|c|c|c|c|c|c|c|c|c|c|c|}
\hline $\begin{array}{l}E \\
x \\
p \\
\#\end{array}$ & $\begin{array}{l}\mathrm{S} \\
\mathrm{t} \\
\mathrm{e} \\
\mathrm{p} \\
\#\end{array}$ & Medium & $\begin{array}{l}\text { Diese } \\
1 \text { Fuel } \\
\text { Conc } \\
(\mathrm{ppm})\end{array}$ & $\begin{array}{l}\text { Potential } \\
\text { TPH } \\
\text { Degraders } \\
\text { Isolated }\end{array}$ & $\begin{array}{l}\text { Cells/g } \\
\text { based } \\
\text { on } \\
\text { Plate } \\
\text { Counts }\end{array}$ & $\begin{array}{l}\text { Naphth } \\
\text {-alene } \\
\text { Conc } \\
\text { (ppm) }\end{array}$ & $\begin{array}{l}\text { Potential } \\
\text { PAH } \\
\text { Degraders } \\
\text { Isolated }\end{array}$ & $\begin{array}{l}\text { Cells/g } \\
\text { based } \\
\text { on } \\
\text { Plate } \\
\text { Counts }\end{array}$ & $\begin{array}{l}\text { PCB } 1 \\
\text { Conc } \\
(\mathrm{ppb})\end{array}$ & $\begin{array}{l}\text { Potential } \\
\text { PCB } \\
\text { Degraders } \\
\text { Isolated }\end{array}$ & $\begin{array}{l}\text { Cells/g } \\
\text { based } \\
\text { on } \\
\text { Plate } \\
\text { Counts }\end{array}$ & $\begin{array}{l}\text { DBZ } \\
\text { Conc } \\
(p p b)\end{array}$ & $\begin{array}{l}\text { Potential } \\
\text { Dioxin } \\
\text { Degraders } \\
\text { Isolated }\end{array}$ & $\begin{array}{l}\text { Cells/g } \\
\text { based } \\
\text { on } \\
\text { Plate } \\
\text { Counts }\end{array}$ & $\begin{array}{l}\text { Total } \\
\text { Incub } \\
\text {-ation } \\
\text { Time }\end{array}$ \\
\hline $\begin{array}{l}1 \\
0\end{array}$ & 1 & $\begin{array}{c}\text { CFMM } \\
\text { Liquid + } \\
\text { MCs }\end{array}$ & 1000 & $x$ & $x$ & 500 & $x$ & $x$ & 100 & $x$ & $x$ & 100 & $x$ & $x$ & $\begin{array}{c}5 \\
\text { weeks }\end{array}$ \\
\hline $\begin{array}{l}1 \\
0\end{array}$ & 2 & $\begin{array}{l}\mathrm{YM}+ \\
\mathrm{MCs}\end{array}$ & 1000 & 0 & $x$ & 500 & 0 & $x$ & 100 & 0 & $x$ & 100 & 0 & $x$ & $\begin{array}{c}5 \\
\text { weeks }\end{array}$ \\
\hline $\begin{array}{l}1 \\
0\end{array}$ & 3 & $\begin{array}{c}\text { YM } \\
\text { Liquid }\end{array}$ & 0 & $x$ & $x$ & 0 & $x$ & $x$ & 0 & $x$ & $x$ & 0 & $x$ & $x$ & $\begin{array}{c}1 \\
\text { week }\end{array}$ \\
\hline $\begin{array}{l}1 \\
1\end{array}$ & 1 & $\begin{array}{c}\text { CFMM } \\
\text { Liquid + } \\
\text { MCs }\end{array}$ & 10000 & $x$ & $x$ & 5000 & $x$ & $x$ & 1000 & $x$ & $x$ & 1000 & $x$ & $x$ & $\begin{array}{c}5 \\
\text { weeks }\end{array}$ \\
\hline $\begin{array}{l}1 \\
1 \\
\end{array}$ & 2 & $\begin{array}{l}\mathrm{YM}+ \\
\mathrm{MCs}\end{array}$ & 10000 & 0 & $x$ & 5000 & 0 & $x$ & 1000 & 0 & $x$ & 1000 & 0 & $x$ & $\begin{array}{c}5 \\
\text { weeks } \\
\end{array}$ \\
\hline $\begin{array}{l}1 \\
1 \\
\end{array}$ & 3 & $\begin{array}{c}\text { YM } \\
\text { Liquid }\end{array}$ & 0 & $x$ & $x$ & 0 & $x$ & $x$ & 0 & $x$ & $x$ & 0 & $x$ & $x$ & $\begin{array}{c}1 \\
\text { week }\end{array}$ \\
\hline $\begin{array}{l}1 \\
2\end{array}$ & 1 & $\begin{array}{c}\text { YM } \\
\text { Liquid + } \\
\text { MCs } \\
\end{array}$ & 100 & $x$ & $x$ & 50 & $x$ & $x$ & 10 & $x$ & $x$ & 10 & $x$ & $x$ & $\begin{array}{c}5 \\
\text { weeks }\end{array}$ \\
\hline $\begin{array}{l}1 \\
2 \\
\end{array}$ & 2 & YM Plate & 100 & 0 & $x$ & 50 & 0 & $x$ & 10 & 0 & $x$ & 10 & 0 & $x$ & $\begin{array}{c}5 \\
\text { weeks } \\
\end{array}$ \\
\hline $\begin{array}{l}1 \\
2 \\
\end{array}$ & 3 & $\begin{array}{c}\text { YM } \\
\text { Liquid }\end{array}$ & 0 & $x$ & $x$ & 0 & $x$ & $x$ & 0 & $x$ & $x$ & 0 & $x$ & $x$ & $\begin{array}{c}1 \\
\text { week }\end{array}$ \\
\hline $\begin{array}{l}1 \\
3\end{array}$ & 1 & $\begin{array}{c}\text { YM } \\
\text { Liquid + } \\
\text { MCs }\end{array}$ & 1000 & $x$ & $x$ & 500 & $x$ & $x$ & 100 & $x$ & $x$ & 100 & $x$ & $x$ & $\begin{array}{c}5 \\
\text { weeks }\end{array}$ \\
\hline $\begin{array}{l}1 \\
3 \\
\end{array}$ & 2 & YM Plate & 1000 & 0 & $x$ & 500 & 0 & $x$ & 100 & 0 & $x$ & 100 & 0 & $x$ & $\begin{array}{c}5 \\
\text { weeks }\end{array}$ \\
\hline $\begin{array}{l}1 \\
3 \\
\end{array}$ & 3 & $\begin{array}{c}\text { YM } \\
\text { Liquid }\end{array}$ & 0 & $x$ & $x$ & 0 & $x$ & $x$ & 0 & $x$ & $x$ & 0 & $x$ & $x$ & $\begin{array}{c}1 \\
\text { week }\end{array}$ \\
\hline
\end{tabular}




\begin{tabular}{|c|c|c|c|c|c|c|c|c|c|c|c|c|c|c|c|}
\hline $\begin{array}{l}E \\
x \\
p \\
\#\end{array}$ & $\begin{array}{l}\mathrm{S} \\
\mathrm{t} \\
\mathrm{e} \\
\mathrm{p} \\
\#\end{array}$ & Medium & $\begin{array}{l}\text { Diese } \\
1 \text { Fuel } \\
\text { Conc } \\
(\mathrm{ppm})\end{array}$ & $\begin{array}{l}\text { Potential } \\
\text { TPH } \\
\text { Degraders } \\
\text { Isolated }\end{array}$ & $\begin{array}{l}\text { Cells/g } \\
\text { based } \\
\text { on } \\
\text { Plate } \\
\text { Counts } \\
\end{array}$ & $\begin{array}{l}\text { Naphth } \\
\text {-alene } \\
\text { Conc } \\
\text { (ppm) }\end{array}$ & $\begin{array}{l}\text { Potential } \\
\text { PAH } \\
\text { Degraders } \\
\text { Isolated }\end{array}$ & $\begin{array}{l}\text { Cells/g } \\
\text { based } \\
\text { on } \\
\text { Plate } \\
\text { Counts } \\
\end{array}$ & $\begin{array}{l}\text { PCB 1 } \\
\text { Conc } \\
\text { (ppb) }\end{array}$ & $\begin{array}{l}\text { Potential } \\
\text { PCB } \\
\text { Degraders } \\
\text { Isolated }\end{array}$ & $\begin{array}{l}\text { Cells/g } \\
\text { based } \\
\text { on } \\
\text { Plate } \\
\text { Counts } \\
\end{array}$ & $\begin{array}{l}\text { DBZ } \\
\text { Conc } \\
\text { (ppb) }\end{array}$ & $\begin{array}{l}\text { Potential } \\
\text { Dioxin } \\
\text { Degraders } \\
\text { Isolated }\end{array}$ & $\begin{array}{l}\text { Cells/g } \\
\text { based } \\
\text { on } \\
\text { Plate } \\
\text { Counts } \\
\end{array}$ & $\begin{array}{l}\text { Total } \\
\text { Incub } \\
\text {-ation } \\
\text { Time }\end{array}$ \\
\hline $\begin{array}{l}1 \\
4\end{array}$ & 1 & $\begin{array}{c}\text { YM } \\
\text { Liquid + } \\
\text { MCs }\end{array}$ & 10000 & $x$ & $x$ & 5000 & $x$ & $x$ & 1000 & $x$ & $x$ & 1000 & $x$ & $x$ & $\begin{array}{c}5 \\
\text { weeks }\end{array}$ \\
\hline $\begin{array}{l}1 \\
4\end{array}$ & 2 & YM Plate & 10000 & 0 & $x$ & 5000 & 0 & $x$ & 1000 & 0 & $x$ & 1000 & 0 & $x$ & $\begin{array}{c}5 \\
\text { weeks }\end{array}$ \\
\hline $\begin{array}{l}1 \\
4\end{array}$ & 3 & $\begin{array}{c}\text { YM } \\
\text { Liquid }\end{array}$ & 0 & $x$ & $x$ & 500 & $x$ & $x$ & 0 & $x$ & $x$ & 0 & $x$ & $x$ & $\begin{array}{c}1 \\
\text { week }\end{array}$ \\
\hline
\end{tabular}


Table 5.3 Notes:

MCs: Model Chemicals, CFMM: Carbon Free Mineral Media, TSA: Tryptone Soy Agar, TSB: Tryptone Soy Broth.

Step indicates the sequence of transfers from media to media within a single culturing experiment.

$\mathrm{X}$ indicates that there is no data. This is caused by the cell either being not applicable or not attempted (in the case of the gray cells).

Bold text indicates experiment portions that yielded no growth of organisms or control organisms, indicating possible toxic effects of concentration. Italic text indicate experiment portions that yielded no growth of organisms or control organisms, indicating a possible stunting of growth from low available carbon. 
Table 5.4: Summary of Isolated Organisms

\begin{tabular}{|c|c|c|c|c|}
\hline & TPH & PAHs & PCBs & Dioxin \\
\hline $\begin{array}{c}\text { Bacteria } \\
\text { Isolated }\end{array}$ & 10 & 8 & 9 & 9 \\
\hline Fungi Isolated & 2 & 2 & 2 & 3 \\
\hline
\end{tabular}

PCR was performed on these colonies with both fungal and bacterial primers to ensure that they would be sequenced and identified correctly. This yielded 21 unique organisms, including 14 bacteria and 7 fungi shown in Table 5.5. This included 3 strains of the fungi Phanerochaete chrysosporium and 6 strains of the bacteria Pseudomonas. Ten of the bacteria and 3 of the fungi isolated are known degraders or come from a genus that contains known degraders of the COIs. "Spiked model compound" is the COI that was used to isolate that microbe. A literature search was done for each microbe to see if it has been reported to biodegrade the COI that it was isolated with. If so, it was noted in the “Known to Degrade COI Isolated with?” column in Table 5.5. Query cover indicates the percent of the query sequence that overlaps with the subject sequence. The indent specifies the percent of the subject sequence that overlaps at the beginning of the sequence. It indicates how much sequence could have been lost due to where the primer is located on the gene. E value is a measure of random background noise. It describes the hits one can expect to see by chance when searching a database of a specific size. The closer and $\mathrm{E}$ value is to 0 the more significant the match is. BLAST HIT indicates the top result of the query in the NCBI database. The query cover values were all $98 \%$ or higher for complete sequences, indicating that they are good matches to the database sequences. 
The indent percentages were all above $80 \%$, and most were above $95 \%$. This indicates that most of the sequence was replicated during PCR, leading to more accurate matches in the database. The $\mathrm{E}$ values were all 0 except in 6 cases. In all 6 of the exceptions the $\mathrm{E}$ value was extremely low, indicating that all isolates had significant matches.

TRFLP uses restriction enzymes to cut DNA extracted from microbes into fragments. These restriction enzymes only cut at very specific DNA sequences. Since the $16 \mathrm{~S}$ region of bacteria DNA and ITS regions of fungi DNA are highly variable these cuts will occur in different places for different organisms. The cuts result in fragments of DNA, whose length is specific to that microbe. However, multiple microbes (especially similar or related microbes) can have a similar $16 \mathrm{~S}$ or ITS sequence, resulting in similar or identical fragment lengths. Only DpnII-cut sites were looked for in bacteria sequences and only HaeIII cut sites for fungi. This is because the TRFLP data only had DpnII digests for bacteria and only HaeIII digests for fungi. It would not be possible to compare HaeIII data for bacteria or DpnII data for fungi to our TRFLP data. The results of the comparison between the isolated microbes predicted TRFLP patterns and the actual fragment data is discussed in Section 5.8.

Other Considerations:

Referring back to Tables 5.2 and 5.3, there are many culturing experiments that yielded no growth at all. This could be due to either toxic effects from high concentrations of COIs or lack of carbon from low concentrations of COIs. Bold text indicates experiment portions that yielded no growth of organisms or control organisms, indicating possible toxic effects of COI concentration. Italic text indicate experiment portions that yielded no 
growth of organisms or control organisms, indicating a possible stunting of growth from low available carbon.

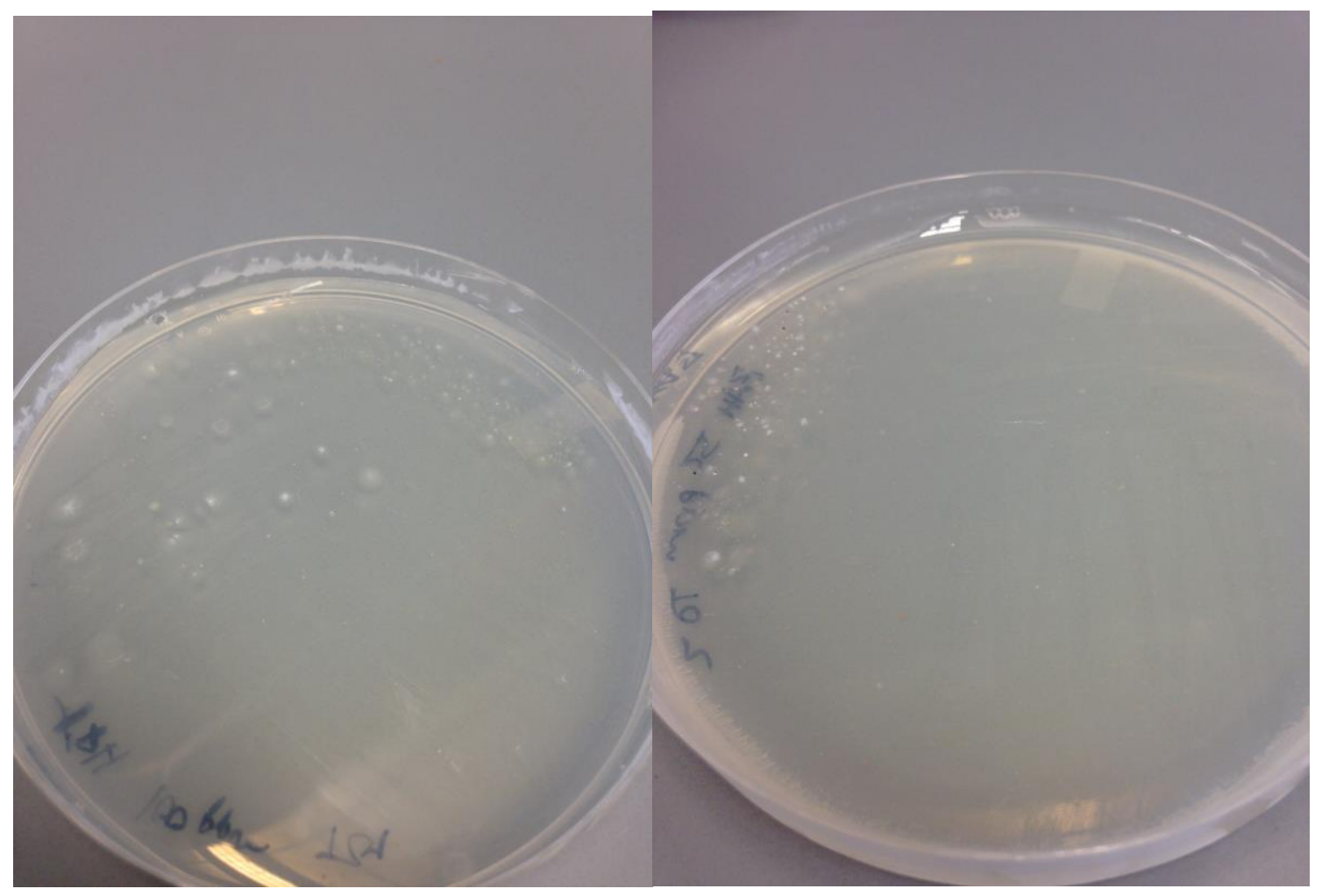

Figure 5.2: Some Plates with Colonies. The left image is a Bushnell Haas plate inoculated with soil from site D02 and spiked with $1 \mathrm{ppm}$ of DBZ showing fungal and bacterial colonies. The right image is a Bushnell Haas plate inoculated with soil from site T02 and spiked with $500 \mathrm{ppm}$ of naphthalene showing fungal colonies. 
Table 5.5: Identified Organisms from Culturing Experiments

\begin{tabular}{|c|c|c|c|c|c|c|c|c|c|c|c|}
\hline $\begin{array}{l}\text { Sample } \\
\text { Name }\end{array}$ & $\begin{array}{c}\text { Spiked } \\
\text { Model } \\
\text { Compound }\end{array}$ & $\begin{array}{l}\text { Sample } \\
\text { Origin }\end{array}$ & $\begin{array}{l}\text { Sequence } \\
\text { Length }\end{array}$ & BLAST HIT & Indent & $\begin{array}{l}\text { Query } \\
\text { Cover }\end{array}$ & $\begin{array}{c}\mathrm{E} \\
\text { value }\end{array}$ & $\begin{array}{l}\text { DpnII } \\
\text { Fragment } \\
\text { Length }\end{array}$ & $\begin{array}{l}\text { HaeIII } \\
\text { Fragment } \\
\text { Length }\end{array}$ & $\begin{array}{c}\text { Known } \\
\text { to } \\
\text { Degrade } \\
\text { COI } \\
\text { Isolated } \\
\text { with? }\end{array}$ & Ref \\
\hline D4 & N/A & $\begin{array}{l}\text { S. wittichii } \\
\text { control }\end{array}$ & 1346 & $\begin{array}{c}\text { Sphingomonas } \\
\text { wittichii }\end{array}$ & $99 \%$ & $100 \%$ & 0 & 129 & N/A & N/A & $\begin{array}{l}\text { T. R. Miller et al. } \\
2010\end{array}$ \\
\hline D3 & N/A & $\begin{array}{l}\text { P. putida } \\
\text { KF715 } \\
\text { control } \\
\end{array}$ & 1371 & Pseudomonas sp & $99 \%$ & $100 \%$ & 0 & 181 & N/A & N/A & $\begin{array}{c}\text { Hayase, Taira, and } \\
\text { Furukawa } 1990\end{array}$ \\
\hline G2 & N/A & $\begin{array}{c}P . \\
\text { naphthalen } \\
\text { ovorans } \\
\text { control } \\
\end{array}$ & 1402 & Paenibacillus sp & $95 \%$ & $98 \%$ & 0 & 210 & N/A & N/A & Daane et al. 2002 \\
\hline D2 & N/A & $\begin{array}{c}R . \\
\text { rhodochrou } \\
s \text { control }\end{array}$ & 1436 & $\begin{array}{l}\text { Rhodocoicus } \\
\text { pyridinivorans }\end{array}$ & $98 \%$ & $99 \%$ & 0 & 514 & N/A & N/A & Sorkhoh et al. 1990 \\
\hline G1 & N/A & $\begin{array}{c}P . \\
\text { chrysospori } \\
\text { um I } \\
\text { control }\end{array}$ & 653 & $\begin{array}{l}\text { Phanerochaete } \\
\text { chrysosporium }\end{array}$ & $96 \%$ & $100 \%$ & 0 & N/A & 574 & N/A & $\begin{array}{c}\text { Fernández-Sánchez } \\
\text { et al. 2001; } \\
\text { Fernández- } \\
\text { Luqueño et al. } \\
\text { 2011; Pérez- } \\
\text { Armendáriz et al. } \\
\text { 2012; Hammel, } \\
\text { Kalyanaraman, and } \\
\text { Kirk 1986; } \\
\text { Hammel 1992 } \\
\end{array}$ \\
\hline I5 & N/A & $\begin{array}{c}P . \\
\text { chrysospori } \\
\text { um } \mathrm{PR} \\
\text { control }\end{array}$ & 781 & $\begin{array}{c}\text { Phanerochaete } \\
\text { chrysosporium } \\
\text { strain KCTC } 6728\end{array}$ & $99 \%$ & $100 \%$ & 0 & N/A & 58 & N/A & N/A \\
\hline
\end{tabular}




\begin{tabular}{|c|c|c|c|c|c|c|c|c|c|c|c|}
\hline $\begin{array}{l}\text { Sample } \\
\text { Name }\end{array}$ & $\begin{array}{c}\text { Spiked } \\
\text { Model } \\
\text { Compound }\end{array}$ & $\begin{array}{l}\text { Sample } \\
\text { Origin }\end{array}$ & $\begin{array}{l}\text { Sequence } \\
\text { Length }\end{array}$ & BLAST HIT & Indent & $\begin{array}{l}\text { Query } \\
\text { Cover }\end{array}$ & $\begin{array}{c}\mathrm{E} \\
\text { value }\end{array}$ & $\begin{array}{c}\text { DpnII } \\
\text { Fragment } \\
\text { Length }\end{array}$ & $\begin{array}{l}\text { HAEIII } \\
\text { Fragment } \\
\text { Length }\end{array}$ & $\begin{array}{c}\text { Known } \\
\text { to } \\
\text { Degrade } \\
\text { COI } \\
\text { Isolated } \\
\text { with? } \\
\end{array}$ & Ref \\
\hline D8 & $\begin{array}{l}\text { \#2 Diesel } \\
\text { Fuel }\end{array}$ & $\mathrm{T} 10$ & 1366 & $\begin{array}{c}\text { Pseudomonas sp. } \\
\text { RKST-1 }\end{array}$ & $99 \%$ & $100 \%$ & 0 & 160 & N/A & $\begin{array}{l}\text { Yes by } \\
\text { spp. }\end{array}$ & $\begin{array}{c}\text { Das and Chandran, } \\
2011\end{array}$ \\
\hline A8 & $\begin{array}{l}\text { \#2 Diesel } \\
\text { Fuel }\end{array}$ & T05 & 1370 & $\begin{array}{c}\text { Pseudomonas sp. } \\
\text { RKST-1 }\end{array}$ & $99 \%$ & $100 \%$ & 0 & 181 & N/A & $\begin{array}{l}\text { Yes by } \\
\text { spp. }\end{array}$ & $\begin{array}{c}\text { Das and Chandran, } \\
2011\end{array}$ \\
\hline $\mathrm{J} 8$ & $\begin{array}{l}\text { \#2 Diesel } \\
\text { Fuel }\end{array}$ & T03 & 1059 & Pseudomonas sp & $94 \%$ & $99 \%$ & 0 & 191 & N/A & Yes & $\begin{array}{c}\text { Das and Chandran, } \\
2011\end{array}$ \\
\hline $\begin{array}{l}\text { F5- } \\
\text { reverse }\end{array}$ & $\begin{array}{l}\text { \#2 Diesel } \\
\text { Fuel }\end{array}$ & T01 & 620 & $\begin{array}{l}\text { Streptomyces } \\
\quad \text { nodosus }\end{array}$ & $83 \%$ & $98 \%$ & $\begin{array}{l}2.00 \mathrm{E} \\
-151\end{array}$ & 541 & N/A & No & N/A \\
\hline K1 & $\begin{array}{l}\text { \#2 Diesel } \\
\text { Fuel }\end{array}$ & T01 & 620 & $\begin{array}{l}\text { Streptomyces } \\
\quad \text { nodosus }\end{array}$ & $83 \%$ & $98 \%$ & $\begin{array}{l}2.00 \mathrm{E} \\
-151\end{array}$ & 541 & N/A & No & N/A \\
\hline K2 & $\begin{array}{l}\text { \#2 Diesel } \\
\text { Fuel }\end{array}$ & T01 & 620 & $\begin{array}{l}\text { Streptomyces } \\
\quad \text { nodosus }\end{array}$ & $83 \%$ & $98 \%$ & $\begin{array}{l}2.00 \mathrm{E} \\
-151\end{array}$ & 541 & N/A & No & N/A \\
\hline $\mathrm{C} 1$ & $\begin{array}{l}\text { \#2 Diesel } \\
\text { Fuel }\end{array}$ & T04 & 1363 & $\begin{array}{l}\text { Streptomyces } \\
\text { flaveolus }\end{array}$ & $99 \%$ & $100 \%$ & 0 & 551 & N/A & No & N/A \\
\hline $\begin{array}{c}\text { F1- } \\
\text { forwar } \\
\text { d }\end{array}$ & $\begin{array}{l}\text { \#2 Diesel } \\
\text { Fuel }\end{array}$ & T07 & 1322 & $\begin{array}{l}\text { Streptomyces } \\
\text { flaveolus }\end{array}$ & $99 \%$ & $100 \%$ & 0 & 551 & N/A & No & N/A \\
\hline
\end{tabular}




\begin{tabular}{|c|c|c|c|c|c|c|c|c|c|c|c|}
\hline $\begin{array}{l}\text { Sample } \\
\text { Name }\end{array}$ & $\begin{array}{l}\text { Spiked } \\
\text { Model } \\
\text { Compound }\end{array}$ & $\begin{array}{l}\text { Sample } \\
\text { Origin }\end{array}$ & $\begin{array}{l}\text { Sequence } \\
\text { Length }\end{array}$ & BLAST HIT & Indent & $\begin{array}{l}\text { Query } \\
\text { Cover }\end{array}$ & $\begin{array}{c}\mathrm{E} \\
\text { value }\end{array}$ & $\begin{array}{c}\text { DpnII } \\
\text { Fragment } \\
\text { Length }\end{array}$ & $\begin{array}{l}\text { HAEIII } \\
\text { Fragment } \\
\text { Length }\end{array}$ & $\begin{array}{c}\text { Known } \\
\text { to } \\
\text { Degrade } \\
\text { COI } \\
\text { Isolated } \\
\text { with? }\end{array}$ & Ref \\
\hline $\mathrm{J} 1$ & $\begin{array}{l}\text { \#2 Diesel } \\
\text { Fuel }\end{array}$ & T08 & 1363 & $\begin{array}{l}\text { Streptomyces } \\
\text { flaveolus }\end{array}$ & $99 \%$ & $100 \%$ & 0 & 551 & N/A & No & N/A \\
\hline $\mathrm{J} 2$ & $\begin{array}{l}\text { \#2 Diesel } \\
\text { Fuel }\end{array}$ & T07 & 1363 & $\begin{array}{l}\text { Streptomyces } \\
\text { flaveolus }\end{array}$ & $99 \%$ & $100 \%$ & 0 & 551 & N/A & No & N/A \\
\hline $\begin{array}{c}\text { I7- } \\
\text { forwar } \\
\mathrm{d} \\
\end{array}$ & $\begin{array}{l}\text { \#2 Diesel } \\
\text { Fuel }\end{array}$ & T01 & 647 & $\begin{array}{c}\text { Phanerochaete } \\
\text { chrysosporium } \\
\text { strain KCTC } 6728\end{array}$ & $92 \%$ & $99 \%$ & 0 & N/A & 56 & No & N/A \\
\hline $\begin{array}{l}\text { I7- } \\
\text { reverse }\end{array}$ & $\begin{array}{l}\text { \#2 Diesel } \\
\text { Fuel }\end{array}$ & T01 & 598 & $\begin{array}{c}\text { Uncultured } \\
\text { Phanerochaete } \\
\text { isolate }\end{array}$ & $83 \%$ & $100 \%$ & $\begin{array}{l}1.00 \mathrm{E} \\
-141\end{array}$ & N/A & 500 & Yes & Yateem et al., 1998 \\
\hline J6 & $\begin{array}{l}\text { \#2 Diesel } \\
\text { Fuel }\end{array}$ & T01 & 598 & $\begin{array}{c}\text { Uncultured } \\
\text { Phanerochaete } \\
\text { isolate }\end{array}$ & $83 \%$ & $100 \%$ & $\begin{array}{l}1.00 \mathrm{E} \\
-141\end{array}$ & N/A & 500 & Yes & Yateem et al., 1998 \\
\hline B1 & Naphthalene & $\mathrm{T} 10$ & 1368 & $\begin{array}{l}\text { Arthrobacter } \\
\text { oxydans }\end{array}$ & $100 \%$ & $100 \%$ & 0 & 97 & N/A & Yes & $\begin{array}{l}\text { Kallimanis et al., } \\
2009\end{array}$ \\
\hline $\mathrm{J} 5$ & Naphthalene & $\mathrm{T} 10$ & 1368 & $\begin{array}{l}\text { Arthrobacter } \\
\text { oxydans }\end{array}$ & $100 \%$ & $100 \%$ & 0 & 97 & N/A & Yes & $\begin{array}{c}\text { Kallimanis et al., } \\
2009\end{array}$ \\
\hline $\mathrm{J} 7$ & Naphthalene & T08 & 1368 & $\begin{array}{l}\text { Arthrobacter } \\
\text { oxydans }\end{array}$ & $100 \%$ & $100 \%$ & 0 & 97 & N/A & Yes & $\begin{array}{l}\text { Kallimanis et al., } \\
2009\end{array}$ \\
\hline $\mathrm{F} 4$ & Naphthalene & T09 & 660 & $\begin{array}{c}\text { Streptomyces } \\
\text { viridochromogene } \\
s\end{array}$ & $92 \%$ & $100 \%$ & 0 & 109 & N/A & $\begin{array}{l}\text { Yes by } \\
\text { spp. }\end{array}$ & $\begin{array}{c}\text { Sutherland et al., } \\
1990\end{array}$ \\
\hline E1 & Naphthalene & T01 & 1368 & $\begin{array}{l}\text { Arthrobacter } \\
\text { oxydans }\end{array}$ & $100 \%$ & $100 \%$ & 0 & 118 & N/A & Yes & $\begin{array}{c}\text { Kallimanis et al., } \\
2009\end{array}$ \\
\hline
\end{tabular}




\begin{tabular}{|c|c|c|c|c|c|c|c|c|c|c|c|}
\hline $\begin{array}{l}\text { Sample } \\
\text { Name }\end{array}$ & $\begin{array}{c}\text { Spiked } \\
\text { Model } \\
\text { Compound }\end{array}$ & $\begin{array}{l}\text { Sample } \\
\text { Origin }\end{array}$ & $\begin{array}{l}\text { Sequence } \\
\text { Length }\end{array}$ & BLAST HIT & Indent & $\begin{array}{l}\text { Query } \\
\text { Cover }\end{array}$ & $\begin{array}{c}\mathrm{E} \\
\text { value }\end{array}$ & $\begin{array}{c}\text { DpnII } \\
\text { Fragment } \\
\text { Length }\end{array}$ & $\begin{array}{l}\text { HaeIII } \\
\text { Fragment } \\
\text { Length }\end{array}$ & $\begin{array}{c}\text { Known } \\
\text { to } \\
\text { Degrade } \\
\text { COI } \\
\text { Isolated } \\
\text { with? }\end{array}$ & Ref \\
\hline B7 & Naphthalene & T02 & 1403 & $\begin{array}{c}\text { Micromonospora } \\
\text { sp. }\end{array}$ & $99 \%$ & $100 \%$ & 0 & 163 & N/A & $\begin{array}{l}\text { Yes in } \\
\text { Consort. }\end{array}$ & $\begin{array}{c}\text { Janbandhu and } \\
\text { Fulekar, } 2011\end{array}$ \\
\hline E7 & Naphthalene & T04 & 1355 & $\begin{array}{c}\text { Micromonospora } \\
\text { chokoriensis }\end{array}$ & $99 \%$ & $100 \%$ & 0 & 1189 & N/A & $\begin{array}{c}\text { Yes, } \\
\text { spp. in } \\
\text { Consort. }\end{array}$ & $\begin{array}{c}\text { Janbandhu and } \\
\text { Fulekar, } 2011\end{array}$ \\
\hline I8 & Naphthalene & T04 & 1355 & $\begin{array}{c}\text { Micromonospora } \\
\text { chokoriensis }\end{array}$ & $99 \%$ & $100 \%$ & 0 & 1189 & N/A & $\begin{array}{c}\text { Yes, } \\
\text { spp. in } \\
\text { Consort. }\end{array}$ & $\begin{array}{c}\text { Janbandhu and } \\
\text { Fulekar, } 2011\end{array}$ \\
\hline $\begin{array}{l}\text { F8- } \\
\text { forwar } \\
\quad \mathrm{d}\end{array}$ & Naphthalene & T07 & 687 & $\begin{array}{l}\text { Phanerochaete } \\
\text { chrysosporium }\end{array}$ & $91 \%$ & $79 \%$ & 0 & N/A & 291 & Yes & Bumpus, 1989 \\
\hline I4 & Naphthalene & T09 & 617 & $\begin{array}{c}\text { Aspergillus } \\
\text { fumigatiaffinis }\end{array}$ & $99 \%$ & $98 \%$ & 0 & N/A & 85 & $\begin{array}{l}\text { Yes by } \\
\text { spp. }\end{array}$ & Varanasi, pg 54 \\
\hline $\begin{array}{l}\text { B4- } \\
\text { reverse }\end{array}$ & PCB \#1 & P10 & 364 & $\begin{array}{c}\text { Pantoea } \\
\text { agglomerans }\end{array}$ & $93 \%$ & $100 \%$ & $\begin{array}{l}2.00 \mathrm{E} \\
-149\end{array}$ & 51 & N/A & No & N/A \\
\hline $\begin{array}{l}\text { E4- } \\
\text { reverse }\end{array}$ & PCB \#1 & P10 & 403 & $\begin{array}{c}\text { Pantoea } \\
\text { agglomerans }\end{array}$ & $97 \%$ & $99 \%$ & 0 & 51 & N/A & No & N/A \\
\hline B2 & PCB \#1 & P01 & 1352 & $\begin{array}{l}\text { Pseudomonas } \\
\text { fluorescens }\end{array}$ & $99 \%$ & $100 \%$ & 0 & 180 & N/A & $\begin{array}{l}\text { Yes by } \\
\text { spp. }\end{array}$ & Du et al., (2001) \\
\hline $\begin{array}{c}\text { B4- } \\
\text { forwar } \\
\text { d }\end{array}$ & PCB \#1 & P10 & 1047 & Pseudomonas sp & $94 \%$ & $99 \%$ & 0 & 180 & N/A & Yes & $\begin{array}{c}\text { Yong-lei et al. } \\
2011\end{array}$ \\
\hline E2 & PCB \#1 & $\mathrm{P} 01$ & 1344 & $\begin{array}{l}\text { Pseudomonas } \\
\text { fluorescens }\end{array}$ & $99 \%$ & $100 \%$ & 0 & 180 & N/A & $\begin{array}{l}\text { Yes by } \\
\text { spp. }\end{array}$ & Du et al., (2001) \\
\hline
\end{tabular}




\begin{tabular}{|c|c|c|c|c|c|c|c|c|c|c|c|}
\hline $\begin{array}{l}\text { Sample } \\
\text { Name }\end{array}$ & $\begin{array}{c}\text { Spiked } \\
\text { Model } \\
\text { Compound }\end{array}$ & $\begin{array}{l}\text { Sample } \\
\text { Origin }\end{array}$ & $\begin{array}{l}\text { Sequence } \\
\text { Length }\end{array}$ & BLAST HIT & Indent & $\begin{array}{l}\text { Query } \\
\text { Cover }\end{array}$ & $\begin{array}{c}\mathrm{E} \\
\text { value }\end{array}$ & $\begin{array}{c}\text { DpnII } \\
\text { Fragment } \\
\text { Length }\end{array}$ & $\begin{array}{l}\text { HaeIII } \\
\text { Fragment } \\
\text { Length }\end{array}$ & $\begin{array}{c}\text { Known } \\
\text { to } \\
\text { Degrade } \\
\text { COI } \\
\text { Isolated } \\
\text { with? }\end{array}$ & Ref \\
\hline $\mathrm{J} 4$ & PCB \#1 & P10 & 1047 & Pseudomonas sp & $94 \%$ & $99 \%$ & 0 & 180 & N/A & Yes & $\begin{array}{l}\text { Yong-lei et al. } \\
2011\end{array}$ \\
\hline $\begin{array}{c}\text { E4- } \\
\text { forwar } \\
d\end{array}$ & PCB \#1 & P10 & 1059 & Pseudomonas sp & $94 \%$ & $99 \%$ & 0 & 191 & N/A & Yes & $\begin{array}{c}\text { Yong-lei et al. } \\
2011\end{array}$ \\
\hline E3 & PCB \#1 & P03 & 1376 & Pseudomonas sp & $96 \%$ & $99 \%$ & 0 & 196 & N/A & Yes & $\begin{array}{c}\text { Yong-lei et al. } \\
2011\end{array}$ \\
\hline B3 & PCB \#1 & P03 & 1374 & Pseudomonas sp & $93 \%$ & $99 \%$ & 0 & 1073 & N/A & Yes & $\begin{array}{c}\text { Yong-lei et al. } \\
2011\end{array}$ \\
\hline $\begin{array}{l}\mathrm{H} 2- \\
\text { reverse }\end{array}$ & PCB \#1 & P01 & 578 & $\begin{array}{c}\text { Aspergillus } \\
\text { fumigatiaffinis }\end{array}$ & $90 \%$ & $95 \%$ & 0 & N/A & 154 & $\begin{array}{l}\text { Yes by } \\
\text { spp. }\end{array}$ & Tigini et al., 2009 \\
\hline I3 & PCB \#1 & P09 & 635 & $\begin{array}{l}\text { Aspergillus } \\
\text { fumigatus }\end{array}$ & $99 \%$ & $99 \%$ & 0 & N/A & 64 & Yes & Tigini et al., 2009 \\
\hline B8 & DBZ & D07 & 1376 & $\begin{array}{l}\text { Variovorax } \\
\text { paradoxus }\end{array}$ & $99 \%$ & $100 \%$ & 0 & 91 & N/A & $\begin{array}{c}\text { Yes, } \\
\text { dechlori } \\
\text { nated }\end{array}$ & Kaiya et al., 2012 \\
\hline E8 & DBZ & D10 & 1378 & $\begin{array}{l}\text { Variovorax } \\
\text { paradoxus }\end{array}$ & $99 \%$ & $100 \%$ & 0 & 152 & N/A & $\begin{array}{c}\text { Yes, } \\
\text { dechlori } \\
\text { nated }\end{array}$ & Kaiya et al., 2012 \\
\hline $\mathrm{J} 3$ & DBZ & D03 & 1378 & $\begin{array}{l}\text { Variovorax } \\
\text { paradoxus }\end{array}$ & $99 \%$ & $100 \%$ & 0 & 152 & N/A & $\begin{array}{c}\text { Yes, } \\
\text { dechlori } \\
\text { nated }\end{array}$ & Kaiya et al., 2012 \\
\hline A5 & DBZ & D07 & 1370 & $\begin{array}{c}\text { Pseudomonas } \\
\text { koreensis }\end{array}$ & $99 \%$ & $100 \%$ & 0 & 181 & N/A & $\begin{array}{l}\text { Yes by } \\
\text { spp. }\end{array}$ & Du et al., (2001) \\
\hline
\end{tabular}




\begin{tabular}{|c|c|c|c|c|c|c|c|c|c|c|c|}
\hline $\begin{array}{l}\text { Sample } \\
\text { Name }\end{array}$ & $\begin{array}{c}\text { Spiked } \\
\text { Model } \\
\text { Compound }\end{array}$ & $\begin{array}{l}\text { Sample } \\
\text { Origin }\end{array}$ & $\begin{array}{l}\text { Sequence } \\
\text { Length }\end{array}$ & BLAST HIT & Indent & $\begin{array}{l}\text { Query } \\
\text { Cover }\end{array}$ & $\begin{array}{c}\mathrm{E} \\
\text { value }\end{array}$ & $\begin{array}{c}\text { DpnII } \\
\text { Fragment } \\
\text { Length }\end{array}$ & $\begin{array}{l}\text { HaeIII } \\
\text { Fragment } \\
\text { Length }\end{array}$ & $\begin{array}{c}\text { Known } \\
\text { to } \\
\text { Degrade } \\
\text { COI } \\
\text { Isolated } \\
\text { with? }\end{array}$ & Ref \\
\hline A7 & DBZ & D06 & 1367 & $\begin{array}{l}\text { Pseudomonas } \\
\text { fluorescens }\end{array}$ & $99 \%$ & $100 \%$ & 0 & 181 & N/A & $\begin{array}{l}\text { Yes by } \\
\text { spp. }\end{array}$ & Du et al., (2001) \\
\hline D5 & DBZ & D07 & 1370 & Pseudomonas sp & $99 \%$ & $100 \%$ & 0 & 181 & N/A & Yes & Hong et al., (2004) \\
\hline D7 & DBZ & D06 & 1372 & $\begin{array}{l}\text { Pseudomonas } \\
\text { fluorescens }\end{array}$ & $99 \%$ & $99 \%$ & 0 & 185 & N/A & $\begin{array}{l}\text { Yes by } \\
\text { spp. }\end{array}$ & Du et al., (2001) \\
\hline K3 & DBZ & D06 & 1372 & $\begin{array}{l}\text { Pseudomonas } \\
\text { fluorescens }\end{array}$ & $99 \%$ & $99 \%$ & 0 & 185 & N/A & $\begin{array}{l}\text { Yes by } \\
\text { spp. }\end{array}$ & Du et al., (2001) \\
\hline A6 & DBZ & D05 & 1383 & $\begin{array}{l}\text { Pseudomonas sp. } \\
\text { b17 }\end{array}$ & $99 \%$ & $100 \%$ & 0 & 194 & N/A & $\begin{array}{l}\text { Yes by } \\
\text { spp. }\end{array}$ & Hong et al., (2004) \\
\hline D6 & DBZ & D09 & 1394 & $\begin{array}{c}\text { Pseudomonas } \\
\text { fluorescens PfO-1 }\end{array}$ & $99 \%$ & $99 \%$ & 0 & 194 & N/A & $\begin{array}{l}\text { Yes by } \\
\text { spp. }\end{array}$ & Du et al., (2001) \\
\hline H5 & DBZ & D05 & 641 & $\begin{array}{l}\text { Aspergillus } \\
\text { fumigatiaffinis }\end{array}$ & $99 \%$ & $97 \%$ & 0 & N/A & 86 & No & N/A \\
\hline H6 & DBZ & D08 & 691 & $\begin{array}{l}\text { Gongronella } \\
\text { butleri }\end{array}$ & $99 \%$ & $94 \%$ & 0 & N/A & 460 & No & N/A \\
\hline $\mathrm{I} 2$ & DBZ & D07 & 622 & Penicillium sp. & $86 \%$ & $98 \%$ & 0 & N/A & 69 & Yes & $\begin{array}{l}\text { Shetty, Zheng, and } \\
\text { Levin, } 1999\end{array}$ \\
\hline
\end{tabular}




\subsection{Culturing Analysis}

Ten different bacteria and 5 different fungi are known biodegraders or belong to genera that contain biodegraders of the COIs at SSFL. Of those 10 bacteria, 8 were identified at least to the species level, but none of these species themselves have been reported as biodegraders of the COIs that they were isolated on. This does not mean that they are not biodegraders necessarily. Because all of these bacteria come from genera that contain biodegraders of the COIs it is possible that they too contain these genes and just have not been studied yet.

One such likely case of this was Pseudomonas, which was isolated 17 times on every COI except PAHs. Of these 17 there were 6 different BLAST hits, suggesting that they are distinct strains or species. Although none of the specific strains of Pseudomonas isolated are known biodegraders of the COIs that they were isolated on, Pseudomonas are very common soil bacteria with many different strains and a broad range of growth substrates (Juteau et al. 1999). Various pseudomonas species biodegrade TPH constituents (Das and Chandran 2011), PCBs (Hong et al. 2004), and dioxin (Hong et al. 2004). It is possible that some of the strains isolated have the capacity to biodegrade the COIs they were isolated for, but they just have not been studied and reported on.

Of the 7 different fungi isolated, 5 were identified to the species or strain level. Of these 5 only 2 were known degraders of the COI used to isolate them. These 2 were Aspergillus fumigatus (PCB \#1) and Phanerochaete chrysosporium (isolated with naphthalene). Tigini et al. (2009) showed that certain strains of Aspergillus fumigatus have the capability to biodegrade PCBs with the addition of glucose. Another Aspergillus species isolated was Aspergillus fumigatiaffinis, which was isolated on DBZ and naphthalene. 
Phanerochaete chrysosporium has been shown to biodegrade PAHs (Bumpus 1989) as well as TPH constituents (Pérez-Armendáriz et al. 2012), PCBs (Fernández-Sánchez et al. 2001), and dioxins (Hammel, Kalyanaraman, and Kirk 1986). There were 2 other identified Phanerochaete isolates, one uncultured strain and one strain that is not a known degrader. However, similar to the case with bacteria, it is possible that these isolates have biodegrading capabilities, which have not yet been published. Although these culturing experiments indicate there are indeed microbes growing in the soil of the site with the capacity to biodegrade model chemicals similar to the COIs, it is important to remember that these are idealized conditions. The model chemicals selected are significantly easier to biodegrade than the COIs, themselves (with the exception of TPH modeled by No. 2 diesel fuel). Additionally, these microbes were streaked onto plates with only the model chemical as a carbon source. This gives the biodegraders of the COIs a significant advantage over other microbes. This is not the case in the soil, where there are many carbon sources and many bacteria that may be able to out-compete the biodegrading microbes. Interactions between bacteria, fungi, and plants, which are also not considered in these experiments, are sure to have effects on microbial populations. These factors will be taken into account in the bioremediation and phytoremediation companion studies currently underway, which both use microcosms with native soil. On the other hand, the soil microbes may grow better in their natural soil environment than they do on laboratory plates.

\subsection{Results of TRFLP Analysis: Bacteria}

Raw TRFLP data comes out of the CEQ 8000 as a chromatogram (see Figure 5.3). Each peak represents a different fragment length. The height of each peak indicates the relative 
abundance of that fragment in that sample. The blue lines are the fragments from the DNA sample. The red lines are fragments from a standard solution. These standards are used for QA/QC purposes. For both sets of fragment data (bacterial and fungi), fragment data from each of the 30 samples was processed and compiled into an excel spreadsheet (see Section 4.3 for details).

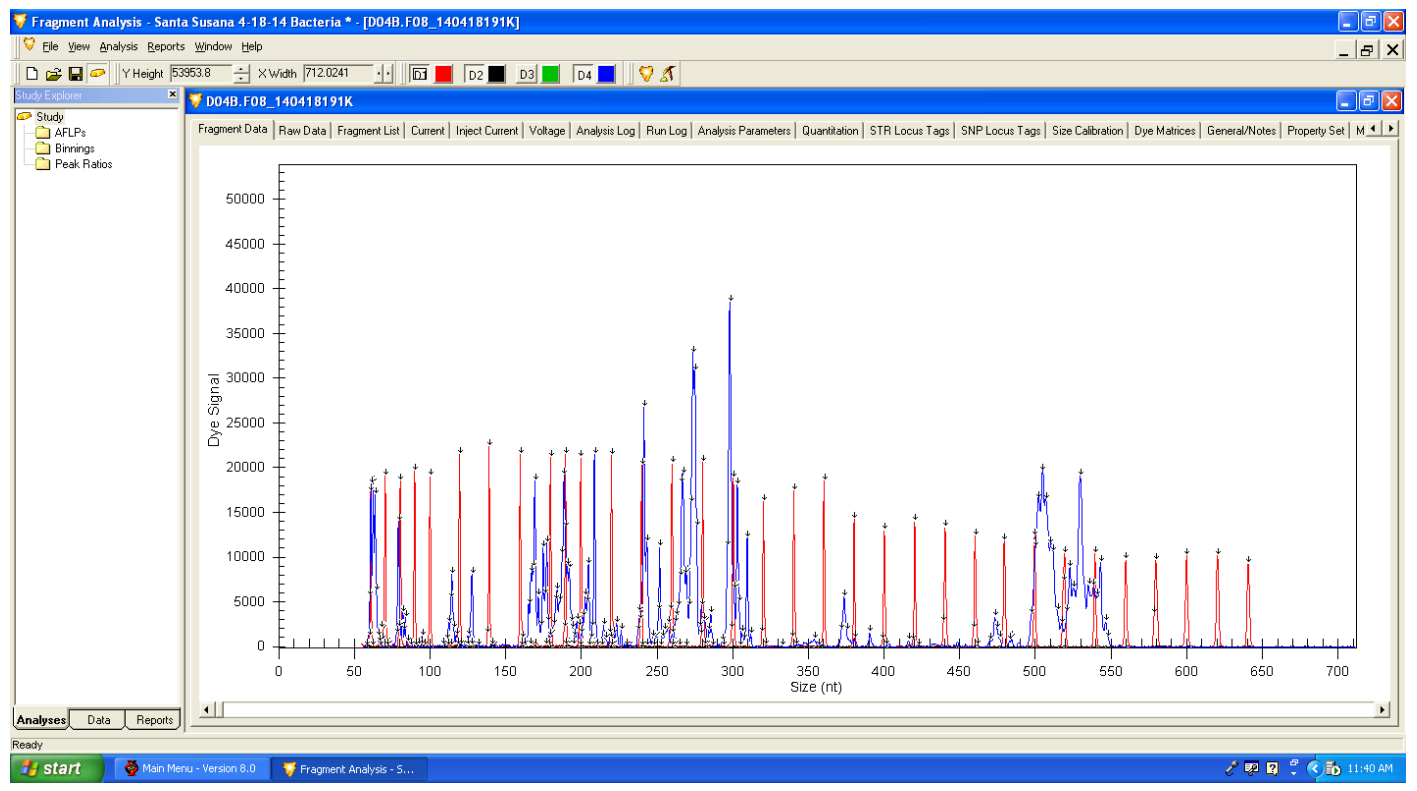

Figure 5.3: An example of a TRFLP chromatogram

The program Primer 5 was used to produce a similarity matrix using the processed fragment analysis for bacteria. A similarity matrix is a matrix of values that represent the how similar each data point is to each other data point. This matrix is used to describe the relative difference between each sample. The magnitude of these differences show how similar or dissimilar microbial communities in these soil samples are. These differences were visualized using multidimensional scaling (MDS). MDS is a method of visualizing data based on differences, and was used to visualize the differences between samples. Because the placement of the data points is based on relative similarity to other data 
points there are no specific parameters or scales associated with the axes. Points are placed based on the relative distance between their respective fragment data, so the chart can be rotated in any direction without changing this distance (Borg and Groenen 1997). For this reason axis labels are normally left off. Factors can then be added to label the data points of the MDS plot in different ways. For this analysis the factors of COI series, soil type, location, presence of TPH, presence of PAHs, presence of PCBs, and presence of dioxin were used. COI series was defined by which sample set the sample came from (i.e. TPH/PAH, dioxin, or PCBs). Table 5.6 details these factors for each sample. Presence of TPH was determined by a threshold of $350 \mathrm{ppm}$. Presence of PAHs was determined by a threshold of $2.5 \mathrm{ppm}$. Presence of PCBs was determined by a threshold of $450 \mathrm{ppb}$. Presence of dioxin was determined by a threshold of $5 \mathrm{ppb}$. Table 5.1 has the specific concentrations of each COI for each sample. Location was determined by the relative positions of the sample sites shown in Figure 5.1. The MDS scatter plot for bacterial data was labeled using different factors to reveal possible patterns. 
Table 5.6: Factors used in MDS Analysis of TRFLP Data

\begin{tabular}{|c|c|c|c|c|c|c|c|}
\hline Sample & COI Series & Soil Type & Location & TPH & PAHs & PCBs & Dioxins \\
\hline T01 & TPH/PAHs & Sandy Loam & Central & $\mathrm{Y}$ & $\mathrm{Y}$ & $\mathrm{Y}$ & $\mathrm{N}$ \\
\hline T02 & TPH/PAHs & Sandy Loam & Central & $\mathrm{Y}$ & $\mathrm{Y}$ & $\mathrm{N}$ & $\mathrm{N}$ \\
\hline T03 & TPH/PAHs & Sandy Loam & Central & $\mathrm{N}$ & $\mathrm{Y}$ & $\mathrm{N}$ & $\mathrm{Y}$ \\
\hline T04 & TPH/PAHs & Sand & Central & $\mathrm{Y}$ & $\mathrm{Y}$ & $\mathrm{N}$ & $\mathrm{N}$ \\
\hline T05 & TPH/PAHs & Silt & South & $\mathrm{N}$ & $\mathrm{Y}$ & $\mathrm{N}$ & $\mathrm{Y}$ \\
\hline T06 & TPH/PAHs & Silt Loam & North & $\mathrm{Y}$ & $\mathrm{Y}$ & $\mathrm{N}$ & $\mathrm{N}$ \\
\hline T07 & TPH/PAHs & Sandy Loam & Central & $\mathrm{N}$ & $\mathrm{Y}$ & $\mathrm{N}$ & $\mathrm{N}$ \\
\hline T08 & TPH/PAHs & Clay & South & $\mathrm{N}$ & $\mathrm{Y}$ & $\mathrm{N}$ & $\mathrm{N}$ \\
\hline T09 & TPH/PAHs & Sandy Loam & Central & $\mathrm{Y}$ & $\mathrm{N}$ & $\mathrm{N}$ & $\mathrm{N}$ \\
\hline $\mathrm{T} 10$ & TPH/PAHs & Sandy Loam & Central & $\mathrm{Y}$ & $\mathrm{Y}$ & $\mathrm{N}$ & $\mathrm{N}$ \\
\hline $\mathrm{P} 01$ & PCBs & Sandy Loam & North & $\mathrm{Y}$ & $\mathrm{N}$ & $\mathrm{Y}$ & $\mathrm{Y}$ \\
\hline $\mathrm{P} 02$ & PCBs & Sandy Loam & North & $\mathrm{N}$ & $\mathrm{N}$ & $\mathrm{Y}$ & $\mathrm{N}$ \\
\hline $\mathrm{P} 03$ & PCBs & Clay & South & - & - & $\mathrm{Y}$ & - \\
\hline P04 & PCBs & Silt & North & $\mathrm{Y}$ & $\mathrm{Y}$ & $\mathrm{Y}$ & $\mathrm{N}$ \\
\hline $\mathrm{P} 05$ & PCBs & Silt Loam & North & $\mathrm{Y}$ & $\mathrm{N}$ & $\mathrm{Y}$ & $\mathrm{N}$ \\
\hline P06 & PCBs & Silt Loam & Central & $\mathrm{Y}$ & $\mathrm{Y}$ & $\mathrm{Y}$ & $\mathrm{N}$ \\
\hline $\mathrm{P} 07$ & PCBs & Sandy Loam & South & - & - & $\mathrm{Y}$ & - \\
\hline P08 & PCBs & Clay & South & - & - & $\mathrm{Y}$ & - \\
\hline P09 & PCBs & Clay & South & - & - & $\mathrm{Y}$ & - \\
\hline P10 & PCBs & Clay & South & - & - & $\mathrm{Y}$ & - \\
\hline D01 & Dioxin & Clay Loam & Central & - & $\mathrm{N}$ & $\mathrm{N}$ & $\mathrm{Y}$ \\
\hline D02 & Dioxin & Silt & South & - & - & - & $\mathrm{Y}$ \\
\hline D03 & Dioxin & Silt & Central & $\mathrm{N}$ & $\mathrm{Y}$ & $\mathrm{N}$ & $\mathrm{Y}$ \\
\hline D04 & Dioxin & Silt Loam & Central & $\mathrm{N}$ & $\mathrm{Y}$ & $\mathrm{N}$ & $\mathrm{Y}$ \\
\hline D05 & Dioxin & Sandy Loam & North & $\mathrm{Y}$ & $\mathrm{N}$ & $\mathrm{N}$ & $\mathrm{Y}$ \\
\hline D06 & Dioxin & Sandy Loam & Central & - & - & $\mathrm{N}$ & $\mathrm{Y}$ \\
\hline D07 & Dioxin & Sand & Central & $\mathrm{N}$ & $\mathrm{Y}$ & $\mathrm{N}$ & $\mathrm{Y}$ \\
\hline D08 & Dioxin & Sandy Loam & North & $\mathrm{N}$ & $\mathrm{N}$ & $\mathrm{N}$ & $\mathrm{Y}$ \\
\hline D09 & Dioxin & Sandy Loam & South & $\mathrm{N}$ & $\mathrm{Y}$ & $\mathrm{N}$ & $\mathrm{Y}$ \\
\hline D10 & Dioxin & Sandy Loam & Central & $\mathrm{N}$ & $\mathrm{Y}$ & $\mathrm{N}$ & $\mathrm{Y}$ \\
\hline
\end{tabular}

An example of a scatter plot comparing the TRFLP patterns based on the factors in Table 5.6 is shown in Figure 5.4, and the remainder are shown in Appendix B. Figure 5.4 shows an MDS scatterplot for bacterial fragment data labeled with the COI of the sample set 
that each sample came from. The factors used for the other scatter plots (shown in Appendix B) were COI series (Figure B.1), location (Figure B.2), soil type, (Figure B.3), presence of TPH (Figure B.4), presence of PAHs (Figure B.5), presence of PCBs (Figure B.6), and presence of dioxin (Figure B.7). The stress value, which is a measure of how reliable the representation is, is shown in the upper right corner of each plot. A stress value of 0.25 or less means the data is well represented.

Primer 5 was again used to calculate the indices of multivariate dispersion between each label within each factor, shown in Table 5.7. This index value must be between -0.05 and 0.05 to be significant. This determines if there is a significant difference between the microbial populations of two different labels based on a group of factors. For example, within the factor of COI series, the index value for $\mathrm{T}$ and $\mathrm{P}$ is -0.17 . This indicates that there is not a significant difference between the 10 samples in the TPH/PAH set and the 10 samples in the PCB sample set. There was no significant correlation between any of these factors and the bacterial populations except in soil type. There was a significant difference in the microbial populations of samples that had sandy loam soil and those that had clay soil (and index value of -.024). The difference in microbial communities between samples that were above the $450 \mathrm{ppb}$ threshold for PCBs compared to those below the threshold was almost significant (index value of 0.06 ). 


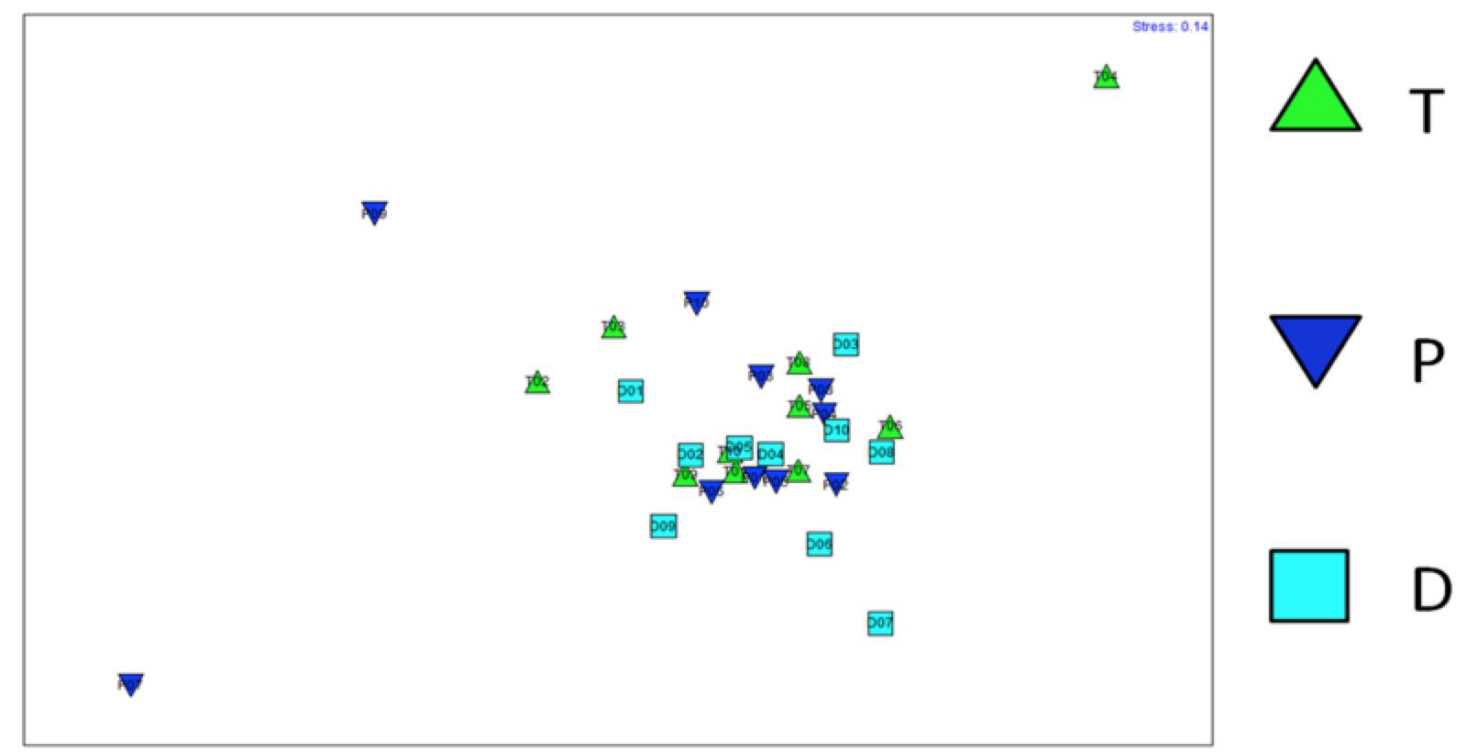

Figure 5.4: MDS from bacterial fragments using COI series as a factor. $\mathrm{T}$ is for the TPH/PAH sample set, $P$ is for the PCB sample set, and D is for the dioxin sample set. 
Table 5.7: Indices of Multivariate Dispersion for each Factor: Bacteria

\begin{tabular}{|c|c|c|}
\hline Factor & Two Groups Compared & $\begin{array}{c}\text { Index of } \\
\text { Multivariate } \\
\text { Dispersion }\end{array}$ \\
\hline COI series & TPH and PAHs, PCBs & -0.17 \\
\hline COI series & TPH and PAHs, Dioxins & 0.161 \\
\hline COI series & PCBs, Dioxins & 0.18 \\
\hline Location & Central, South & -0.138 \\
\hline Location & Central, North & 0.699 \\
\hline Location & South, North & 0.677 \\
\hline Soil Type & Sandy Loam, Sand & -0.802 \\
\hline Soil Type & Sandy Loam, Silt & 0.308 \\
\hline Soil Type & Sandy Loam, Silty Loam & 0.377 \\
\hline Soil Type & Sandy Loam, Clay & -0.024 \\
\hline Soil Type & Sand, Silt & 1 \\
\hline Soil Type & Sand, Silty Loam & 1 \\
\hline Soil Type & Sand, Clay & 1 \\
\hline Soil Type & Silt, Silty Loam & 0.167 \\
\hline Soil Type & Silt, Clay & -0.267 \\
\hline Soil Type & Silty Loam, Clay & -0.367 \\
\hline P/A of TPH & Yes, No & -0.069 \\
\hline P/A of PAHs & Yes, No & 0.286 \\
\hline P/A of PCBs & Yes, No & 0.06 \\
\hline P/A of & Yes, No & -0.14 \\
\hline Dioxins & & \\
\hline
\end{tabular}

Legend: $\mathrm{T}=$ Sample from the TPH or PAHs sample sets, $\mathrm{P}=$ Sample from the PCBs sample set, $\mathrm{D}=$ Sample from the Dioxins sample set, $\mathrm{C}=\mathrm{Central}, \mathrm{N}=$ North, $\mathrm{S}=$ South, $\mathrm{Y}=\mathrm{Yes}, \mathrm{N}=\mathrm{No}$

Possible effects of contaminant concentration on the bacterial community were visualized by adding bubbles to each sample, with bubble size proportional to the 
concentration of the contaminant being investigated in that analysis. For these bubble plots only individual sample sets of 10 were used in order minimize "noise" from the other 20 samples. The other samples are represented on the scatter plots as " 0 ”s. They are important because they can show the similarity between the samples with bubbles (which are within that set of 10 soil samples) and the other soil samples (which are not in the set of 10). This was performed for all 4 COIs (Figure 5.5 for TPH, Figure 5.6 for PAHs, Figure 5.7 for PCBs, and Figure 5.8 for dioxin). Figure 5.7 represents the log scale PCB concentration in order to give the other data more visibility. If there was a significant effect of COI concentration the high concentration samples would be grouped together away from the lower concentration samples. However, in this study the samples with high and low COI concentrations were not grouped away from each other for any COIs. This indicates that the concentrations of COIs did not have any reproducible effect on the TRFLP pattern, and thus did not likely impact the microbial community population dynamics. Although the log scale PCB scatterplot show that most high concentrations are grouped together, they are not grouped away from either the low concentration bubbles nor the soil samples from the other 2 sets of soil samples. This means that although the microbial communities in the PCB soil samples are similar, they are also similar to most of the other microbial communities in other soil samples with less PCBs. 


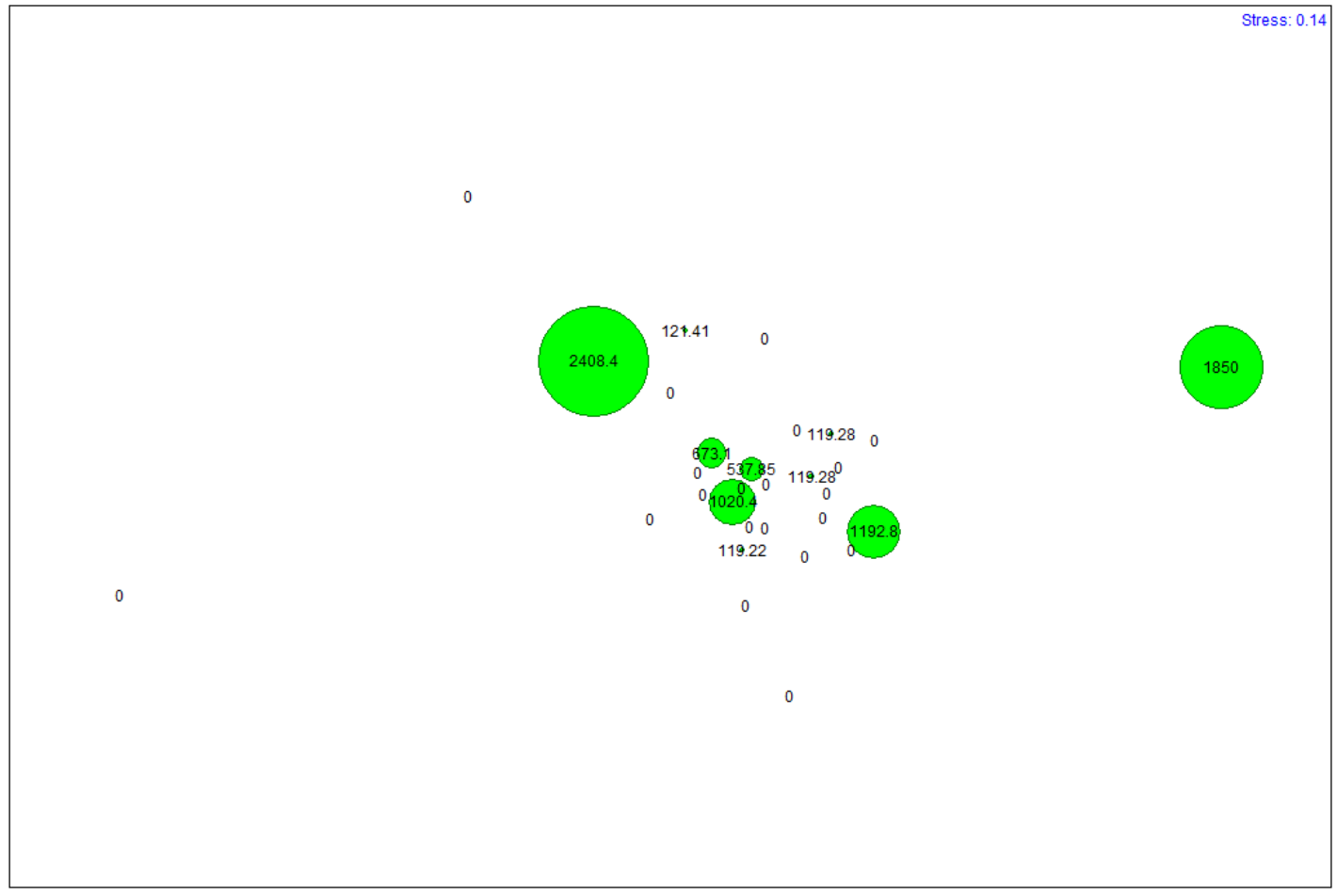

Figure 5.5: MDS from bacterial fragments with TPH bubbles based on TPH concentrations

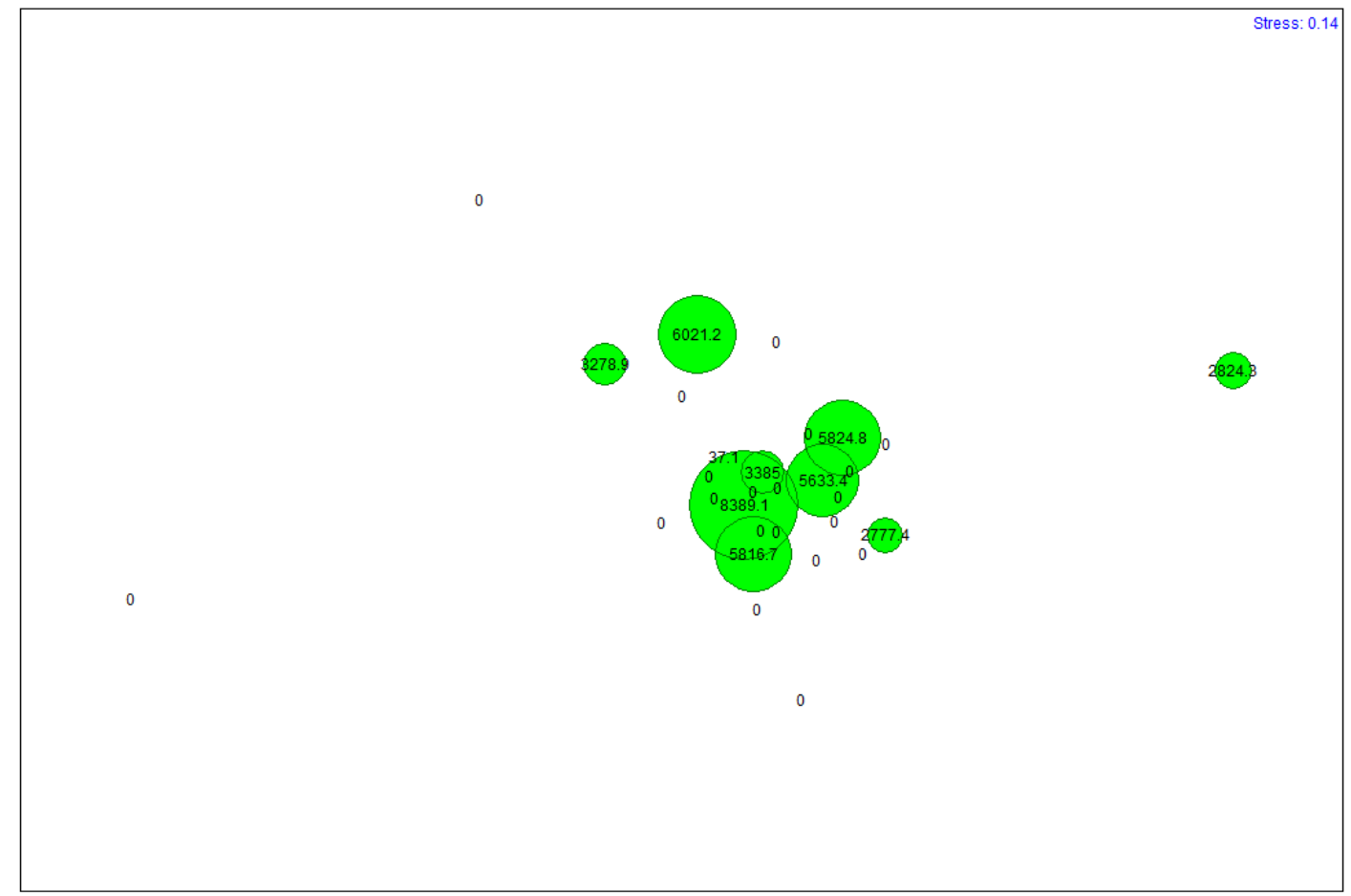

Figure 5.6: MDS from bacterial fragments with PAH bubbles based on concentrations of PAHs. 


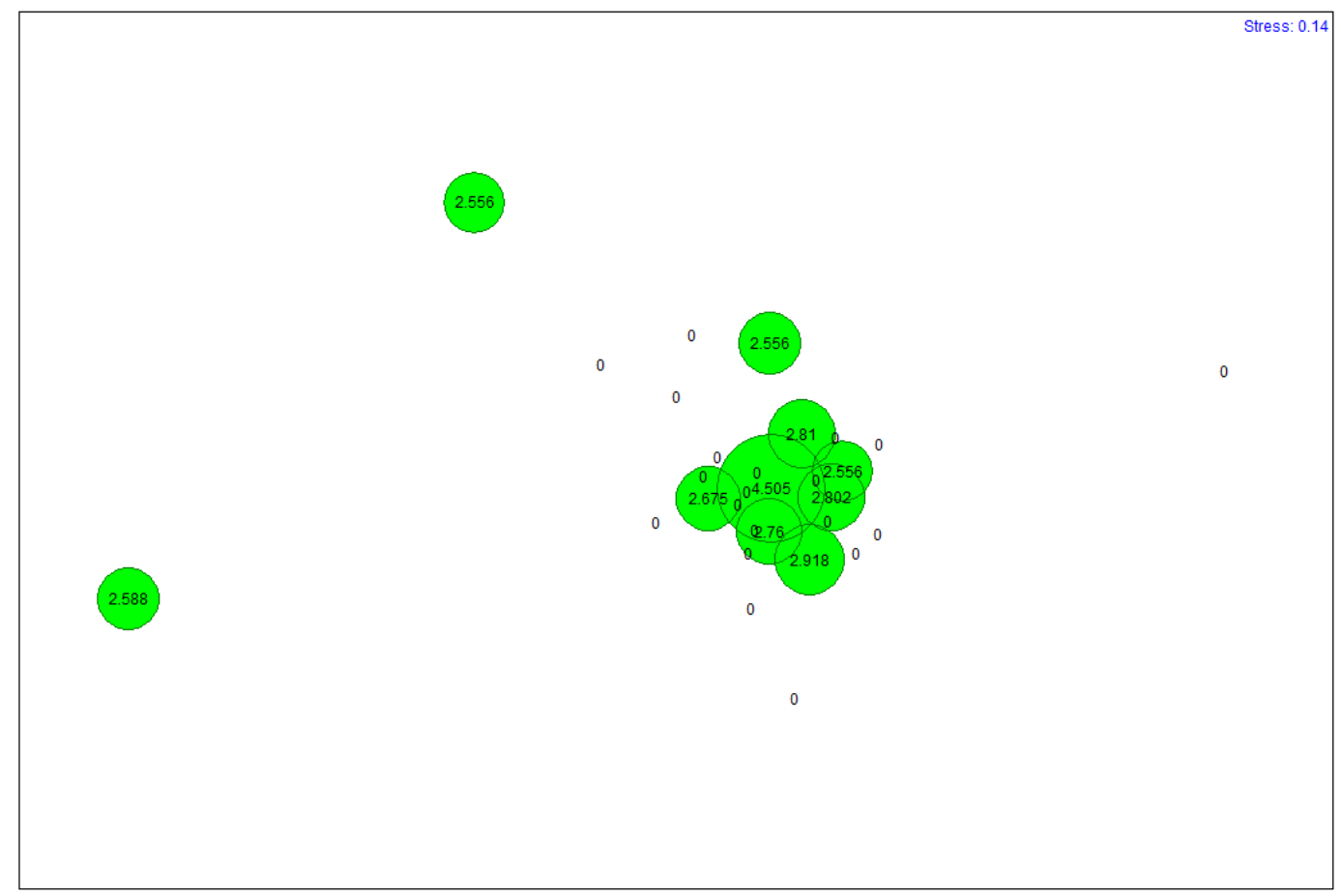

Figure 5.7: MDS from bacterial fragments with PCBs bubbles based on log of PCB concentrations

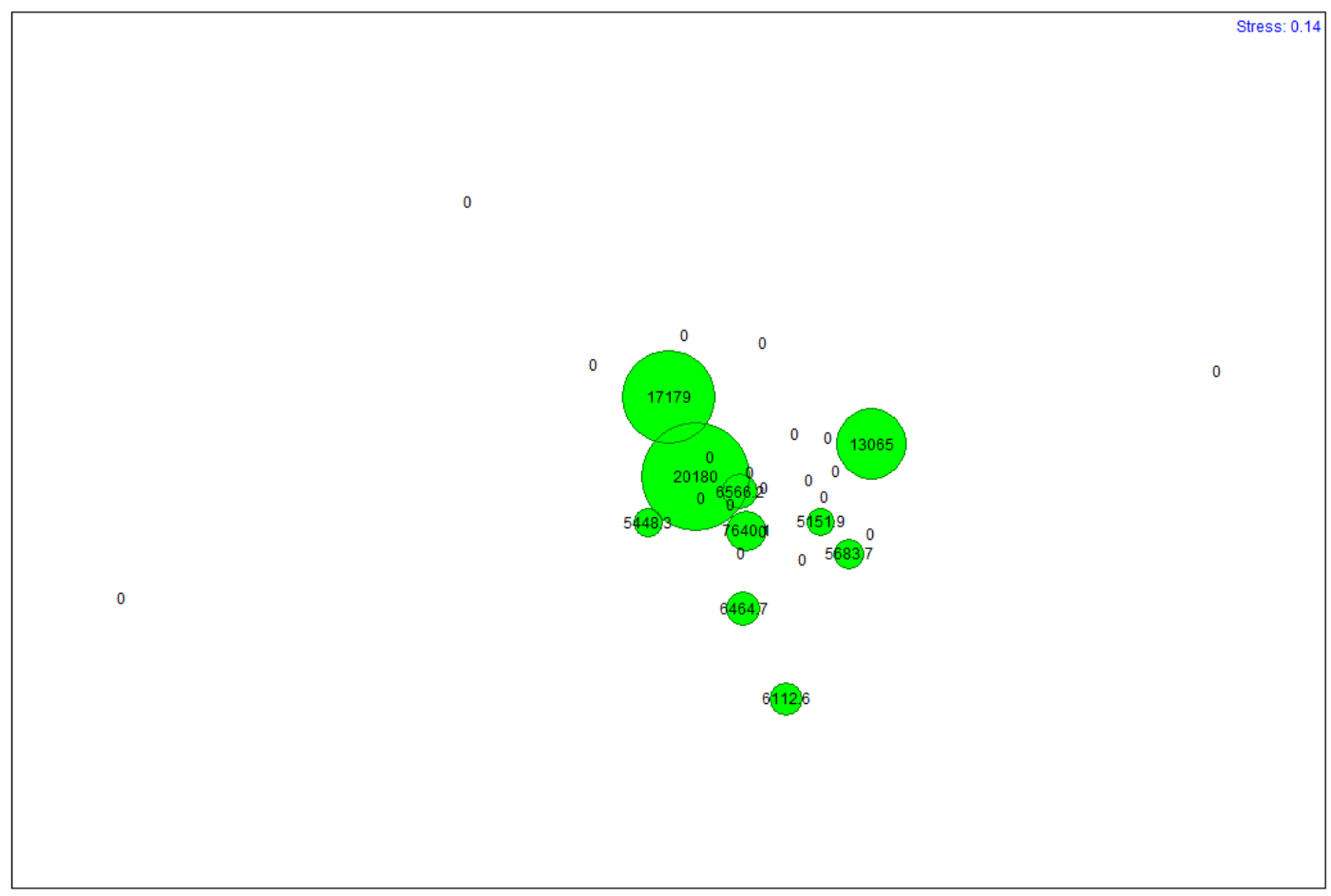

Figure 5.8: MDS from bacterial fragments with dioxin bubbles based on dioxin concentrations 


\subsection{Results of TRFLP Analysis: Fungi}

Just like for bacterial data, COI series was defined by which sample set the sample came from (i.e. TPH/PAH, dioxin, or PCBs). Table 5.6 details these factors for each sample. Again the factors used were COI series (Figure B.8), location (Figure B.9), soil type, (Figure B.10), presence of TPH (Figure B.11), presence of PAHs (Figure B.12), presence of PCBs (Figure B.13), and presence of dioxin (Figure B.14). The MDS scatter plots for these factors can be found in Appendix B. The indices of multivariate dispersion are shown in Table 5.8. There was no significant correlation between any of these factors and the bacterial populations. Unlike with the bacterial data, the soil type seemed to have no discernable effect on microbial population dynamics. 
Table 5.8: Indices of Multivariate Dispersion for each Factor: Fungi

\begin{tabular}{|c|c|c|c|}
\hline $\begin{array}{c}\text { Bacteria/ } \\
\text { Fungi }\end{array}$ & Factor & Two Groups Compared & $\begin{array}{c}\text { Index of } \\
\text { Multivariate } \\
\text { Dispersion }\end{array}$ \\
\hline Fungi & COl Series & TPH and PAHs, PCBs & -0.224 \\
\hline Fungi & COl Series & TPH and PAHs, Dioxins & 0.235 \\
\hline Fungi & COI Series & PCBs, Dioxins & 0.406 \\
\hline Fungi & Location & Central, South & -0.298 \\
\hline Fungi & Location & Central, North & 0.298 \\
\hline Fungi & Location & South, North & 0.466 \\
\hline Fungi & Soil Type & Sandy Loam, Sand & 0.824 \\
\hline Fungi & Soil Type & Sandy Loam, Silt & -0.15 \\
\hline Fungi & Soil Type & Sandy Loam, Silty Loam & -0.136 \\
\hline Fungi & Soil Type & Sandy Loam, Clay & -0.756 \\
\hline Fungi & Soil Type & Sand, Silt & -0.667 \\
\hline Fungi & Soil Type & Sand, Silty Loam & -0.667 \\
\hline Fungi & Soil Type & Sand, Clay & -0.8 \\
\hline Fungi & Soil Type & Silt, Silty Loam & -0.167 \\
\hline Fungi & Soil Type & Silt, Clay & -0.767 \\
\hline Fungi & Soil Type & Silty Loam, Clay & -0.733 \\
\hline Fungi & P/A of TPH & Yes, No & 0.192 \\
\hline Fungi & P/A of PAHs & Yes, No & -0.358 \\
\hline Fungi & P/A of PCBs & Yes, No & 0.26 \\
\hline Fungi & P/A of Dioxins & Yes, No & 0.143 \\
\hline
\end{tabular}

Legend: $\mathrm{T}=$ Sample from the TPH or PAHs sample sets, $\mathrm{P}=$ Sample from the PCBs sample set, $\mathrm{D}=$ Sample from the Dioxins sample set, $\mathrm{C}=$ Central, $\mathrm{N}=$ North, $\mathrm{S}=$ South, $\mathrm{Y}=\mathrm{Yes}, \mathrm{N}=\mathrm{No}$

As was done for bacteria, possible effects of contaminant concentrations on the fungal community were visualized by adding bubbles to each sample that are proportional to the concentration of the contaminant being investigated in that analysis. This was performed 
for all 4 COIs (Figure 5.9 for TPH, Figure 5.10 for PAHs, Figure 5.11 for PCBs, and Figure 5.12 for dioxin). Figure 5.11 represents the log scale PCB concentration bubbles in order to give the other data more visibility. None of these concentrations had significant correlations to microbial populations.

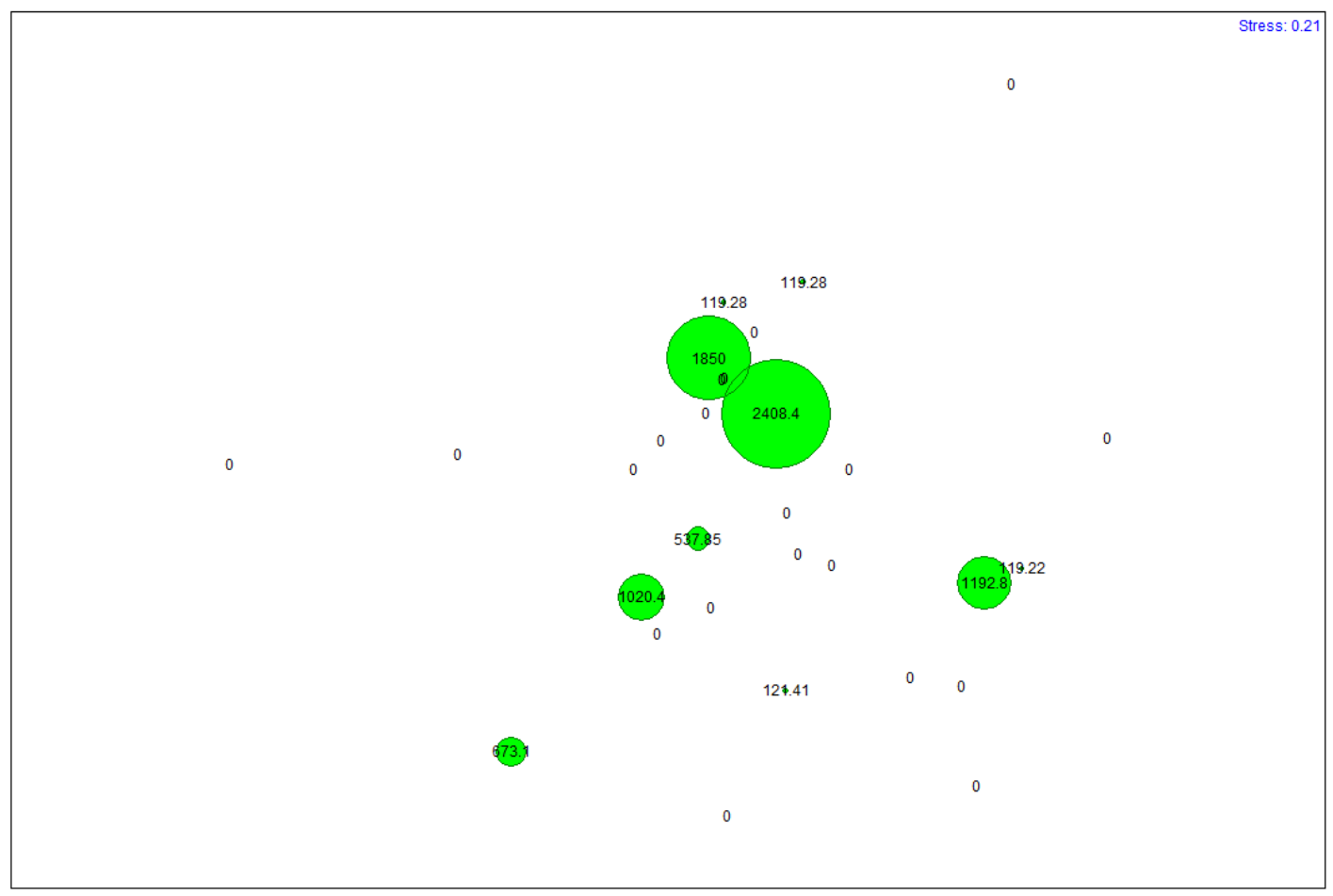

Figure 5.9: MDS from fungal fragments with TPH bubbles based on TPH concentrations. 


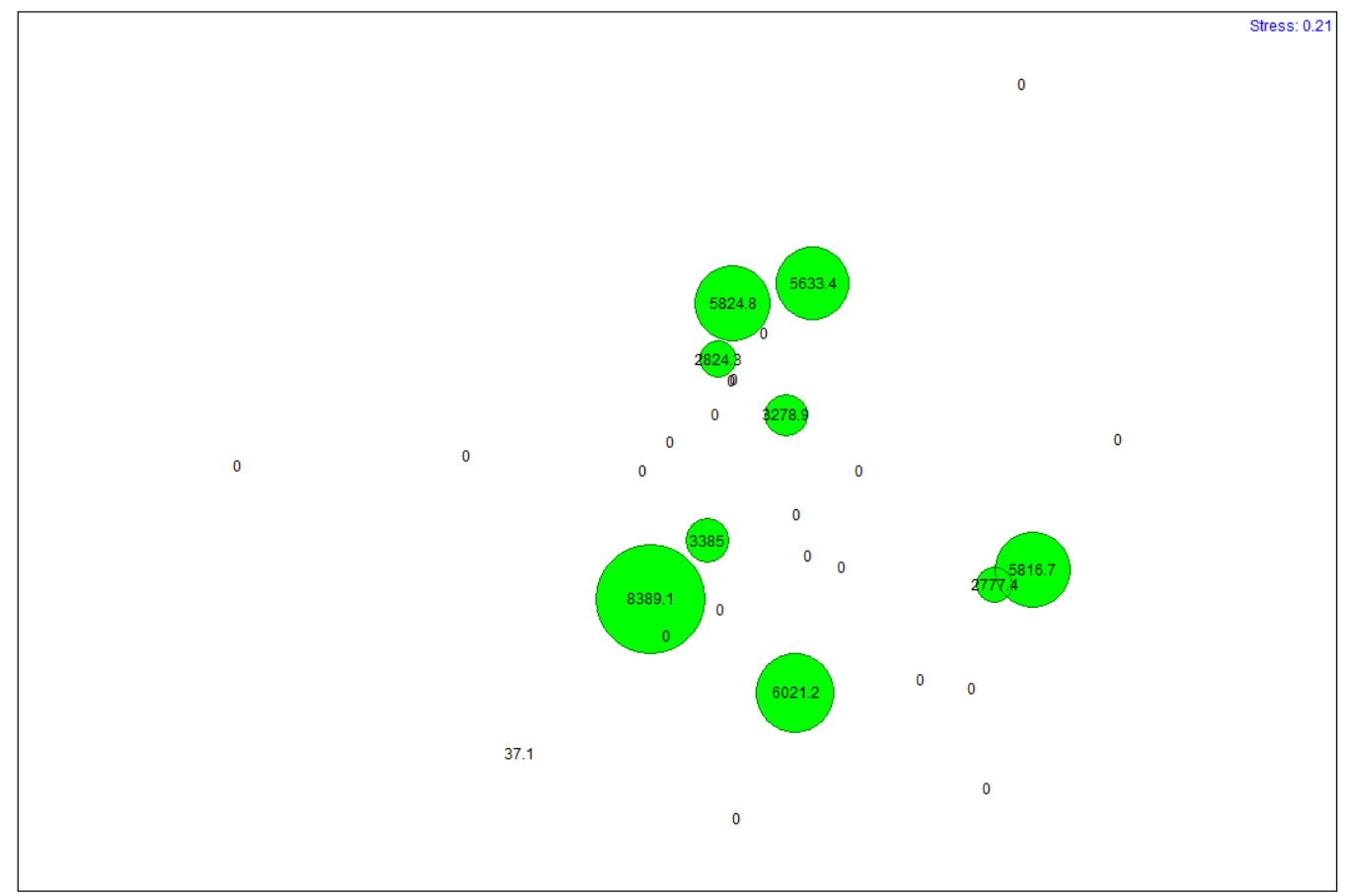

Figure 5.10: MDS from fungal fragments with PAH bubbles based on concentrations of PAHs

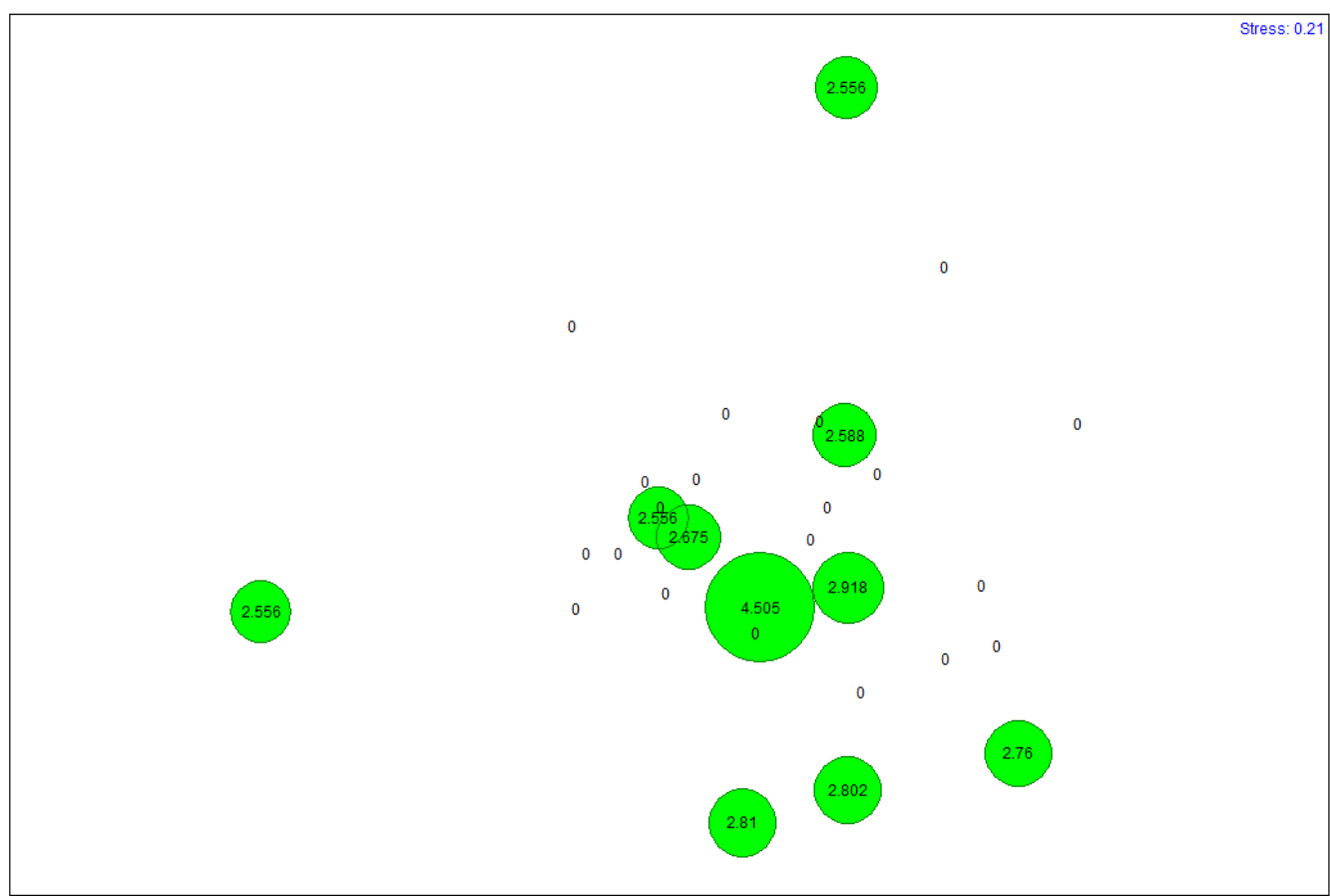

Figure 5.11: MDS from fungal fragments with PCBs bubbles based on log of PCB concentrations 


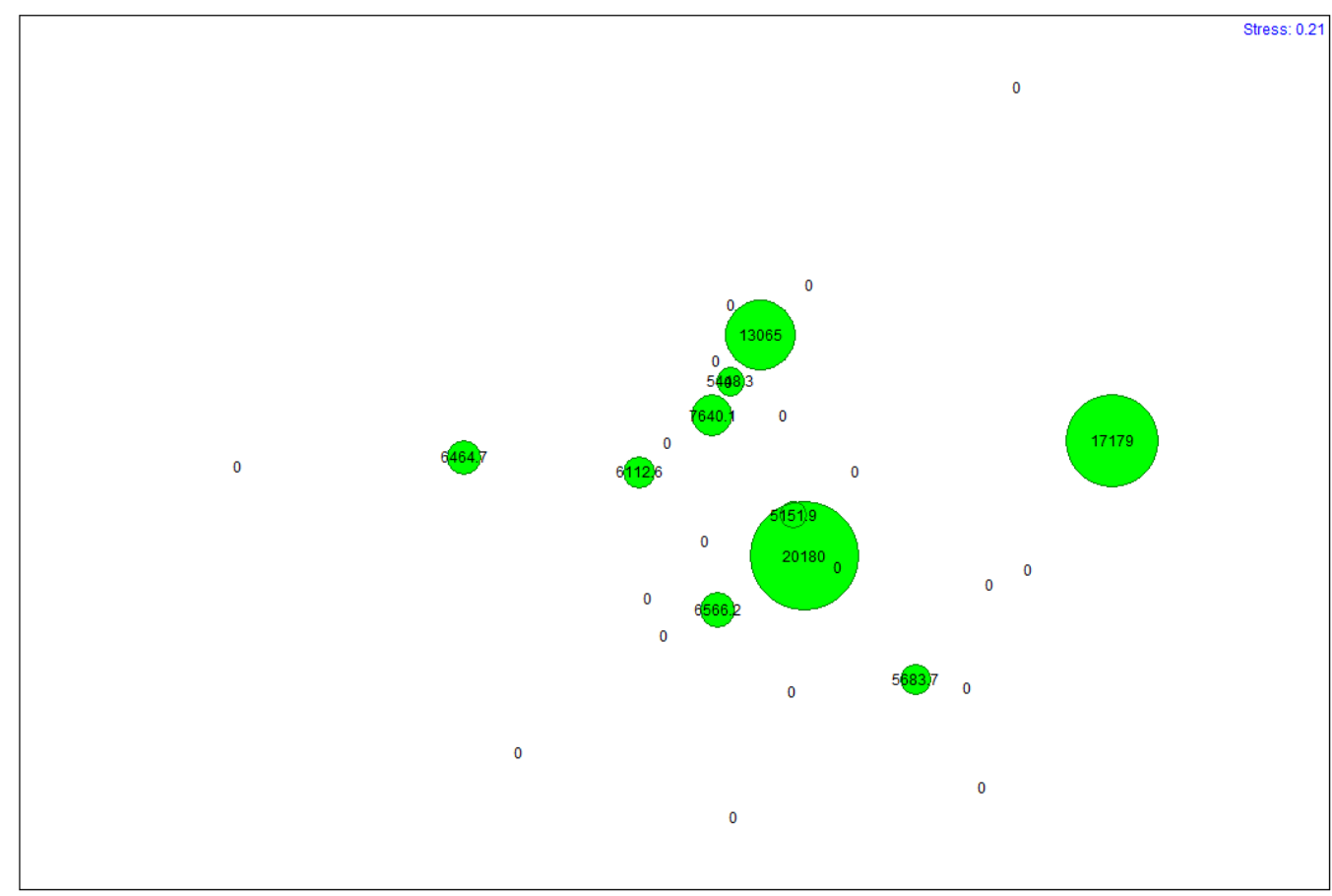

Figure 5.12: MDS from fungal fragments with dioxin bubbles based on dioxin concentrations

\subsection{TRFLP MDS and Indices of Multivariate Dispersion Analysis}

There was only one significant correlation based on the MDS scatterplots and multivariate dispersion indices for both bacterial and fungal data, which was between clay and sandy loam soil types for bacteria. The difference in microbial communities of soil samples that had PCBs above the threshold and those that did not was nearly significant for bacteria. This suggests that the physical characteristics of the soil have the most impact on the microbial population dynamics. This may have to do with moisture, as different soil types will hold different amounts of moisture (Nicholson and Farrar 1994). Certainly moisture and other characteristics of the soil are known to have an effect on the microbial community in soil (Staley and Reysenbach 2002). Results of the fungal fragment analysis were similar with no correlation observed between COI sample set, 
location, soil type, presence of TPH, presence of PAHs, presence of PCBs, or presence of dioxin and the fragment data.

Although the difference in TRFLP patterns of soil samples that had PCBs above the threshold and those that did not was not significant for bacteria, it was close enough to infer that PCBs may have an effect on bacteria in the soil. The index value was 0.06 , which was only 0.01 away from being significant. PCBs are some of the most recalcitrant and toxic chemicals of the COIs, and therefore may be causing an impact on the bacterial community dynamics.

An important consideration when interpreting the TRFLP data is that all the COIs are broadly dispersed in the soil. Every soil sample site had every COI that was tested for in the soil. This muddled the data, making it very hard to pick out correlations of TRFLP peaks with particular COIs. Because there are no correlations between the fragment analyses and the presence of any one COI, the microbial communities must be relatively similar. This does not mean that there are no biodegraders on the site; it just means that if they are present they are likely to be broadly dispersed.

For dioxins and PCBs, correlations were not expected between TRFLP patterns and the presence of these COIs because of site conditions. The soils at SSFL are highly aerobic, making anaerobic dechlorination impossible in the bulk of the soil. Reductive dechlorination may be occurring in anaerobic microenvironments in the soil but the amount of bacteria that inhabit these pockets are not likely be strongly represented in a TRFLP pattern. Additionally, concentrations of PCBs and dioxins even at the most contaminated locations were not high enough to give a degrading microbe a significant 
advantage over other bacteria. The highest PCB concentrations were mostly below 650 $\mathrm{ppb}$ (with a few exceptions) and the highest dioxin concentrations were around $20 \mathrm{ppb}$. If there are degraders of PCBs or dioxins on the site, their populations are not likely large enough to cause a significant change in the total microbial population of that soil.

However, the bacteria communities of soil samples with PCBs above the threshold were close to different. This may be caused by toxic effects at a few sample sites with extreme PCB levels rather than the presence of biodegraders. There were a couple sites that had extremely high PCB concentrations relative to the majority of the sample sites. For example, sample P01 had roughly 50 to 1000 times more PCBs than the other samples in the PCB sample set. These few data points may have caused a difference in the bacterial fragment data for PCB sites, leading to them nearly being significantly different to the other samples. Not only are PCBs recalcitrant, especially in an aerobic soil like those of SSFL, they are also highly toxic. It is more likely that the bacterial populations in the soil with high PCBs are comprised of more PCB resistant bacteria than sample sites with low PCBs. This effect may be what caused the difference in bacterial populations between the two groups to be nearly significant.

An important consideration is that TRFLP combined with MDS scatterplots and indices of multivariate dispersion analyses look at microbial communities as a whole and may overlook individual organisms that may be able to biodegrade a COI. If a degrader is present in the soil but does not make up a large portion of the population it won't make that microbial community significantly different from a community that doesn't contain this degrader. To prevent overlooking these potential degraders correlation functions were used to compare TRFLP peaks to COI concentrations. Additionally, TRFLP peaks 
for all isolated potential degraders and control organisms from the culturing experiments were examined to determine if they showed up in the TRFLP fragment data.

\subsection{TRFLP Correlations}

There were 6 peaks ( 3 bacteria and 3 fungi) that had correlations between 0.40 and 0.48 to PCBs. However, on closer examination it was determined that this was not useful information due to the concentration variability of PCBs in the samples. Sample P01 had roughly 50 to 1000 times more PCBs than the other samples. In all 6 cases the peak had correlated with PCBs because of this large concentration difference between those samples and the other 28 . The fragment data revealed that although these peaks mathematically correlated to PCBs, the peaks represented between $2-3.5 \%$ of the total microbial population for that sample. These peaks were in fact small compared to other peaks, but received a bias because they were found in a sample with extremely high PCBs and not often in other samples.

\subsection{Cultured Potential Degraders and Control Organisms in TRFLP Fragment Data}

For each isolated potential degrader and positive control organism the equivalent TRFLP fragment length was determined using the sequence data. These fragments were then searched for in the TRFLP fragment data. For each isolated microbe, the COI it was isolated on, the TRFLP fragment length, the name of the microbe, the samples that contained its TRFLP peak, the relative abundance of this peak in the sample, if it is known to biodegrade the COI it was isolated on, and references are shown in Table 5.9. Because of the possible error from the CEQ machine and the PCR digest, TRFLP peaks that were within 1 base pair of the cultured microbe's predicted fragment length were considered reported in this table. Only 5 of the 21 isolates and control organisms did not 
have their fragment peak appear in any samples. The TRFLP peaks of all 6 positive control organisms were found in the fragment data as well. Of the 22 of isolates and control organisms that did have their fragment peaks appear in the fragment data, 19 of them were potential degraders. 
Table 5.9: Cultured Organisms in TRFLP Fragment Analysis

\begin{tabular}{|c|c|c|c|c|c|c|c|c|c|}
\hline $\begin{array}{l}\text { Bacteria } \\
\text { /Fungi }\end{array}$ & $\begin{array}{c}\text { Spiked } \\
\text { Model } \\
\text { Compound }\end{array}$ & BLAST HIT & $\begin{array}{l}\text { DpnII } \\
\text { Fragment } \\
\text { Length }\end{array}$ & $\begin{array}{l}\text { HaeIII } \\
\text { Fragment } \\
\text { Length }\end{array}$ & $\begin{array}{l}\text { Samples that } \\
\text { Contained } \\
\text { Microbe Based } \\
\text { on Culturing } \\
\text { Experiments } \\
\end{array}$ & $\begin{array}{l}\text { Samples that } \\
\text { Contained } \\
\text { Microbe } \\
\text { Based on } \\
\text { TRFLP } \\
\end{array}$ & $\begin{array}{c}\text { Relative } \\
\text { Abundance } \\
\text { of Microbe } \\
\text { in Soil } \\
\text { Sample } \\
\end{array}$ & $\begin{array}{l}\text { Known to } \\
\text { Degrade } \\
\text { COI Isolated } \\
\text { with? }\end{array}$ & Ref \\
\hline Bacteria & N/A & $\begin{array}{c}\text { Rhodocoicus } \\
\text { pyridinivorans }\end{array}$ & 514 & N/A & $\begin{array}{l}\text { R. rhodochrous } \\
\text { control (PHCs) }\end{array}$ & D06 & $2.0 \%$ & N/A & $\begin{array}{c}\text { Sorkhoh et al. } \\
1990 \\
\end{array}$ \\
\hline Bacteria & N/A & $\begin{array}{c}\text { Paenibacillus } \\
\text { sp }\end{array}$ & 210 & N/A & $\begin{array}{c}P . \\
\text { naphthalenovora } \\
n s \text { control } \\
\text { (PAHs) }\end{array}$ & $\begin{array}{l}\text { T01, T02, } \\
\text { T07, P02, } \\
\text { P05, P09, } \\
\text { D01, D04, } \\
\text { D05, D06 }\end{array}$ & $\begin{array}{l}2.2 \%, 1.5 \% \\
2.4 \%, 1.5 \% \\
2.0 \%, 1.3 \% \\
2.2 \%, 3.5 \% \\
1.7 \%, 3.1 \%\end{array}$ & N/A & Daane et al. 2002 \\
\hline Bacteria & N/A & $\begin{array}{c}\text { Pseudomonas } \\
\text { sp }\end{array}$ & 181 & N/A & $\begin{array}{c}\text { P. putida KF715 } \\
\text { control (PCBs) }\end{array}$ & $\begin{array}{l}\text { P07, P09, } \\
\text { D02, }\end{array}$ & $\begin{array}{c}5.3 \%, 3.4 \% \\
1.6 \%\end{array}$ & N/A & $\begin{array}{c}\text { Hayase, Taira, } \\
\text { and Furukawa } \\
1990\end{array}$ \\
\hline Bacteria & N/A & $\begin{array}{c}\text { Sphingomonas } \\
\text { wittichii }\end{array}$ & 129 & N/A & $\begin{array}{c}\text { S. wittichii } \\
\text { control (Dioxin) }\end{array}$ & D01, D04 & $1.3 \%, 2.1 \%$ & N/A & $\begin{array}{c}\text { T. R. Miller et al. } \\
2010\end{array}$ \\
\hline Fungi & N/A & $\begin{array}{l}\text { Phanerochaete } \\
\text { chrysosporium }\end{array}$ & N/A & 574 & $\begin{array}{c}P . \\
\begin{array}{c}\text { chrysosporium I } \\
\text { control }\end{array}\end{array}$ & P05, D08 & $8.8 \%, 2.1 \%$ & N/A & $\begin{array}{c}\text { Fernández- } \\
\text { Sánchez et al. } \\
2001\end{array}$ \\
\hline Fungi & N/A & $\begin{array}{c}\text { Phanerochaete } \\
\text { chrysosporium } \\
\text { strain KCTC } \\
6728\end{array}$ & N/A & 58 & $\begin{array}{c}P . \\
\text { chrysosporium } \\
\text { PR control }\end{array}$ & $\begin{array}{l}\text { T02, T03, } \\
\text { T09, P02, } \\
\text { P03, P07, } \\
\text { P10, D02, } \\
\text { D04, D05, } \\
\text { D06, D08 }\end{array}$ & $\begin{array}{c}2.3 \%, 5.4 \% \\
5.9 \%, 3.1 \% \\
4.1 \%, 1.4 \% \\
18.7 \% \\
1.5 \%, 1.5 \% \\
1.3 \%, 5.3 \% \\
3.4 \%\end{array}$ & N/A & $\begin{array}{l}\text { Fernández- } \\
\text { Sánchez et al. } \\
2001\end{array}$ \\
\hline Bacteria & Naphthalene & $\begin{array}{c}\text { Arthrobacter } \\
\text { oxydans }\end{array}$ & 97 & N/A & $\begin{array}{c}\text { T01, T08, T10 } \\
(2)\end{array}$ & P09 & $1.3 \%$ & Yes & $\begin{array}{c}\text { Kallimanis et al., } \\
2009\end{array}$ \\
\hline Bacteria & Naphthalene & $\begin{array}{c}\text { Micromonospor } \\
\text { a chokoriensis }\end{array}$ & 1189 & N/A & T04 (2) & N/A* & N/A & $\begin{array}{l}\text { Yes, spp. in } \\
\text { Consort. }\end{array}$ & $\begin{array}{c}\text { Janbandhu and } \\
\text { Fulekar, } 2011 \\
\end{array}$ \\
\hline Bacteria & Naphthalene & $\begin{array}{c}\text { Micromonospor } \\
\text { a sp. }\end{array}$ & 163 & N/A & T02 & P07, P09 & $2.6 \%, 2.7 \%$ & $\begin{array}{c}\text { Yes in } \\
\text { Consort. }\end{array}$ & $\begin{array}{l}\text { Janbandhu and } \\
\text { Fulekar, } 2011\end{array}$ \\
\hline
\end{tabular}




\begin{tabular}{|c|c|c|c|c|c|c|c|c|c|}
\hline $\begin{array}{l}\text { Bacteria } \\
\text { /Fungi }\end{array}$ & $\begin{array}{c}\text { Spiked } \\
\text { Model } \\
\text { Compound }\end{array}$ & BLAST HIT & $\begin{array}{l}\text { DpnII } \\
\text { Fragment } \\
\text { Length }\end{array}$ & $\begin{array}{c}\text { HaeIII } \\
\text { Fragment } \\
\text { Length }\end{array}$ & $\begin{array}{l}\text { Samples that } \\
\text { Contained } \\
\text { Microbe Based } \\
\text { on Culturing } \\
\text { Experiments }\end{array}$ & $\begin{array}{c}\text { Samples that } \\
\text { Contained } \\
\text { Microbe } \\
\text { Based on } \\
\text { TRFLP }\end{array}$ & $\begin{array}{c}\text { Relative } \\
\text { Abundance of } \\
\text { Microbe in } \\
\text { Soil Sample }\end{array}$ & $\begin{array}{c}\text { Known to } \\
\text { Degrade COI } \\
\text { Isolated with? }\end{array}$ & Ref \\
\hline Bacteria & PCB \#1 & $\begin{array}{c}\text { Pantoea } \\
\text { agglomerans }\end{array}$ & 51 & N/A & P10 (2) & N/A* & N/A & No & N/A \\
\hline Bacteria & DBZ & $\begin{array}{c}\text { Pseudomonas } \\
\text { fluorescens }\end{array}$ & 181 & N/A & D06 (3), P01 & $\begin{array}{l}\text { P07, P09, } \\
\text { D02, }\end{array}$ & $\begin{array}{c}5.3 \%, 3.4 \% \\
1.6 \%\end{array}$ & Yes by spp. & Du et al., (2001) \\
\hline Bacteria & DBZ & $\begin{array}{c}\text { Pseudomonas } \\
\text { fluorescens } \\
\text { Pfo-1 }\end{array}$ & 194 & N/A & D09 & $\begin{array}{l}\text { T01, T06, } \\
\text { P01, P04, } \\
\text { P08, P10, } \\
\text { D03, D06, } \\
\text { D08 }\end{array}$ & $\begin{array}{c}1.5 \%, 1.5 \%, \\
1.6 \%, 2.2 \% \\
1.6 \%, 1.5 \% \\
2.0 \%, 2.5 \% \\
3.5 \%\end{array}$ & Yes by spp. & Du et al., (2001) \\
\hline Bacteria & DBZ & $\begin{array}{c}\text { Pseudomonas } \\
\text { koreensis }\end{array}$ & 181 & N/A & D07 & $\begin{array}{l}\text { P07, P09, } \\
\text { D02, }\end{array}$ & $\begin{array}{c}5.3 \%, 3.4 \% \\
1.6 \%\end{array}$ & Yes by spp. & Du et al., (2001) \\
\hline Bacteria & DBZ & $\begin{array}{c}\text { Pseudomonas } \\
\text { sp }\end{array}$ & 181 & N/A & $\begin{array}{l}\text { D07, P03 (2), } \\
\text { P10 (3), T03 }\end{array}$ & $\begin{array}{l}\text { P07, P09, } \\
\text { D02, }\end{array}$ & $\begin{array}{c}5.3 \%, 3.4 \% \\
1.6 \%\end{array}$ & Yes & Hong et al., (2004) \\
\hline Bacteria & DBZ & $\begin{array}{c}\text { Pseudomonas } \\
\text { sp. b17 }\end{array}$ & 194 & N/A & D05 & $\begin{array}{l}\text { T01, T06, } \\
\text { P01, P04, } \\
\text { P08, P10, } \\
\text { D03, D06, } \\
\text { D08 }\end{array}$ & $\begin{array}{c}1.5 \%, 1.5 \% \\
1.6 \%, 2.2 \% \\
1.6 \%, 1.5 \% \\
2.0 \%, 2.5 \% \\
3.5 \%\end{array}$ & Yes by spp. & Hong et al., (2004) \\
\hline Bacteria & $\begin{array}{l}\text { \#2 Diesel } \\
\text { Fuel }\end{array}$ & $\begin{array}{c}\text { Pseudomonas } \\
\text { sp. RKS7-1 }\end{array}$ & 181 & $\mathrm{~N} / \mathrm{A}$ & $\mathrm{T} 05, \mathrm{~T} 10$ & $\begin{array}{l}\text { P07, P09, } \\
\text { D02, }\end{array}$ & $\begin{array}{c}5.3 \%, 3.4 \% \\
1.6 \%\end{array}$ & Yes by spp. & $\begin{array}{c}\text { Das and Chandran, } \\
2011\end{array}$ \\
\hline Bacteria & $\begin{array}{l}\text { \#2 Diesel } \\
\text { Fuel }\end{array}$ & $\begin{array}{l}\text { Streptomyces } \\
\text { flaveolus }\end{array}$ & 551 & N/A & $\begin{array}{c}\text { T04, T07 (2), } \\
\text { T08 }\end{array}$ & none & N/A & No & N/A \\
\hline Bacteria & $\begin{array}{l}\text { \#2 Diesel } \\
\text { Fuel }\end{array}$ & $\begin{array}{l}\text { Streptomyces } \\
\text { nodosus }\end{array}$ & 541 & N/A & T01 (3) & $\begin{array}{l}\text { T05, T06, } \\
\text { T08, D06, } \\
\text { D07, D08, } \\
\text { D10 }\end{array}$ & $\begin{array}{l}2.5 \%, 2.2 \% \\
1.4 \%, 1.9 \% \\
4.7 \%, 3.7 \%\end{array}$ & No & N/A \\
\hline Bacteria & $\begin{array}{c}\text { Naphthalen } \\
\mathrm{e}\end{array}$ & $\begin{array}{c}\text { Streptomyces } \\
\text { viridochromo } \\
\text { genes }\end{array}$ & 109 & N/A & T09 & none & N/A & Yes by spp. & $\begin{array}{c}\text { Sutherland et al., } \\
1990\end{array}$ \\
\hline Bacteria & DBZ & $\begin{array}{l}\text { Variovorax } \\
\text { paradoxus }\end{array}$ & 152 & N/A & $\begin{array}{l}\text { D03, D07, } \\
\text { D10 }\end{array}$ & none & N/A & $\begin{array}{c}\text { Yes, } \\
\text { unchlorinated }\end{array}$ & Kaiya et al., 2012 \\
\hline
\end{tabular}




\begin{tabular}{|c|c|c|c|c|c|c|c|c|c|}
\hline $\begin{array}{l}\text { Bacteria } \\
\text { /Fungi }\end{array}$ & $\begin{array}{c}\text { Spiked } \\
\text { Model } \\
\text { Compound }\end{array}$ & BLAST HIT & $\begin{array}{l}\text { DpnII } \\
\text { Fragment } \\
\text { Length }\end{array}$ & $\begin{array}{l}\text { HaeIII } \\
\text { Fragment } \\
\text { Length }\end{array}$ & $\begin{array}{l}\text { Samples that } \\
\text { Contained } \\
\text { Microbe } \\
\text { Based on } \\
\text { Culturing } \\
\text { Experiments }\end{array}$ & $\begin{array}{l}\text { Samples that } \\
\text { Contained } \\
\text { Microbe } \\
\text { Based on } \\
\text { TRFLP }\end{array}$ & $\begin{array}{c}\text { Relative } \\
\text { Abundance of } \\
\text { Microbe in } \\
\text { Soil Sample }\end{array}$ & $\begin{array}{l}\text { Known to } \\
\text { Degrade } \\
\text { COI Isolated } \\
\text { with? }\end{array}$ & Ref \\
\hline Fungi & DBZ & $\begin{array}{c}\text { Aspergillus } \\
\text { fumigatiaffinis }\end{array}$ & N/A & 86 & $\begin{array}{l}\text { D05, P01, } \\
\text { T09 }\end{array}$ & $\begin{array}{l}\text { T01, T02, } \\
\text { T03, T04, } \\
\text { T05, T06, } \\
\text { T07, T08, } \\
\text { T10, P01, } \\
\text { P02, P03, } \\
\text { P06, P07, } \\
\text { P08, D01, } \\
\text { D02, D03, } \\
\text { D04, D05, } \\
\text { D06, D07, } \\
\text { D09, D10 }\end{array}$ & $\begin{array}{c}8.2 \%, 5.2 \%, \\
34.0 \%, 4.5 \%, \\
2.0 \%, 1.7 \%, \\
1.4 \%, 3.2 \% \\
4.1 \%, 3.5 \% \\
33.5 \%, 4.7 \%, \\
4.7 \%, 7.2 \% \\
3.7 \%, 1.3 \% \\
11.5 \%, 2.1 \% \\
12.7 \%, 5.0 \% \\
4.5 \%, 6.8 \% \\
3.3 \%, 10.7 \%\end{array}$ & No & N/A \\
\hline Fungi & PCB \#1 & $\begin{array}{l}\text { Aspergillus } \\
\text { fumigatus }\end{array}$ & N/A & 64 & P09 & T05 & $3.6 \%$ & Yes & Tigini et al., 2009 \\
\hline Fungi & $\begin{array}{l}\text { \#2 Diesel } \\
\text { Fuel }\end{array}$ & $\begin{array}{c}\text { Uncultured } \\
\text { Phanerochaete } \\
\text { isolate }\end{array}$ & N/A & 500 & T01 (2) & none & N/A & Yes & Yateem et al., 1998 \\
\hline Fungi & DBZ & Penicillium sp. & N/A & 69 & D07 & none & N/A & Yes & $\begin{array}{c}\text { Shetty, Zheng, and } \\
\text { Levin, } 1999\end{array}$ \\
\hline Fungi & $\begin{array}{c}\text { Naphthalen } \\
\text { e }\end{array}$ & $\begin{array}{l}\text { Phanerochaete } \\
\text { chrysosporium }\end{array}$ & N/A & 291 & T07 & Т06, Т07 & $5.7 \%, 5.8 \%$ & Yes & Bumpus, 1989 \\
\hline Fungi & $\begin{array}{l}\text { \#2 Diesel } \\
\text { Fuel }\end{array}$ & $\begin{array}{c}\text { Phanerochaete } \\
\text { chrysosporium } \\
\text { strain KCTC } \\
6728\end{array}$ & N/A & 56 & T01 & T05 & $1.4 \%$ & No & N/A \\
\hline Fungi & DBZ & $\begin{array}{c}\text { Gongronella } \\
\text { butleri }\end{array}$ & N/A & 460 & D08 & P04, D01 & $1.5 \%, 2.8 \%$ & No & N/A \\
\hline
\end{tabular}


Notes for Table 5.9"

* = Possibly out of CEQ 8000's measurable range

(\#) Indicates that a microbe was isolated multiple times from the same soil sample. The number in the brackets indicates the amount of times it was isolated from that sample. 
It is important to remember the presence of one of these peaks in a sample does not indicate that a microbe is definitely present. Many microbes share the same fragment length. However, in the case of the 22 organisms whose TRFLP peaks were reported and that were cultured directly from the soil it is likely that they do exist in SSFL soil and that the peaks do represent them. The 19 reported degraders cover all 4 COIs and include both fungi and bacteria. The majority of these TRLFP peaks are between $1.5 \%$ and $5 \%$ of the samples fragments. Assuming that all samples have an average amount of bacteria count $10^{8}-10^{9}$ bacteria per gram, this represents $1.5 \times 10^{5}$ to $5 \times 10^{7}$ cells per gram. qPCR results support these estimations. Although these are not huge populations, they are still significant for natural attenuation.

Combining the TRFLP and culturing data reveals the distribution of these microbes across the site. Six of the isolates had their TRFLP peak appear in 5 or more of the 30 samples. Four of these were seen in 9 or more of the 30 samples. These microbes are making up a relatively small percentage of the total microbial population, meaning that other non-degraders make up the vast majority of the population. These non-degraders are most likely common soil microbes, which is why the TRFLP patterns between samples were so similar.

\subsection{Results of qPCR}

Table 5.10 gives the number of cells per gram for each target. The qPCR analysis revealed that of the 6 aerobic BTEX degradation targets were detected. Four of these 6 were detected in both samples. The cells per gram that contained these targets varied between $4.8 \times 10^{8}$ to $4.92 \times 10^{4}$. No anaerobic targets in either soil sample with the exception of benzoyl coenzyme A (BCR), which is associated with anaerobic BTEX 
biodegradation. Sample 1 had $8.2 \times 10^{8}$ cells per gram that contained BCR and sample 2 had $7.1 \times 10^{7}$ cells per gram. No aerobic or anaerobic PAH biodegradation targets were detected. Dehalococcoides spp., which is associated with reductive dechlorination of PCBs and dioxins (Bunge and Lechner 2009; Bedard, Ritalahti, and Löffler 2007), was detected in sample 1 (fresh soil from D03), but only in a small amount. In the qPCR analysis each target and shows a quantitative scale relating those numbers to typical observed values. 
Table 5.10 Summary of the QuantArray ${ }^{\circledR}$ Petro qPCR results.

\begin{tabular}{|c|c|c|}
\hline Analysis & $\begin{array}{c}\text { Sample 1 } \\
\text { (from } \\
\text { D03) } \\
\text { (cells/g) }\end{array}$ & $\begin{array}{c}\text { Sample } 2 \\
\text { (composite) } \\
\text { (cells/g) }\end{array}$ \\
\hline Aerobic BTEX and MTBE & - & - \\
\hline Toluene/Benzene Dioxygenase (TOD) & $<1.00 \mathrm{E}+04$ & $<1.00 \mathrm{E}+04$ \\
\hline Phenol Hydroxylase (PHE) & $7.43 \mathrm{E}+06$ & $2.31 \mathrm{E}+05$ \\
\hline $\begin{array}{c}\text { Toluene } 2 \text { Monooxygenase/Phenol Hydroxylase } \\
\text { (RDEG) }\end{array}$ & $2.86 \mathrm{E}+06$ & $6.70 \mathrm{E}+04$ \\
\hline $\begin{array}{l}\text { Toluene Ring Hydroxylating Monooxygenases } \\
\text { (RMO) }\end{array}$ & $7.27 \mathrm{E}+04$ & $<1.00 \mathrm{E}+04$ \\
\hline Xylene/Toluene Monooxygenase (TOL) & $<1.00 \mathrm{E}+04$ & $<1.00 \mathrm{E}+04$ \\
\hline $\begin{array}{l}\text { Ethylbenzene/Isopropylbenzene Dioxygenase } \\
\text { (EDO) }\end{array}$ & $<1.00 \mathrm{E}+04$ & $<1.00 \mathrm{E}+04$ \\
\hline Biphenyl/Isopropylbenzene Dioxygenase (BPH4) & $9.67 \mathrm{E}+04$ & $<1.00 \mathrm{E}+04$ \\
\hline Methylibium petroliphilum PM1 (PM1) & $4.80 \mathrm{E}+08$ & $6.59 \mathrm{E}+06$ \\
\hline TBA Monooxygenase (TBA) & $<1.00 \mathrm{E}+04$ & $4.92 \mathrm{E}+04$ \\
\hline Aerobic PAHs and Alkanes & - & - \\
\hline Naphthalene Dioxygenase (NAH) & $<1.00 \mathrm{E}+04$ & $<1.00 \mathrm{E}+04$ \\
\hline Phenanthrene Dioxygenase (PHN) & $<1.00 \mathrm{E}+04$ & $<1.00 \mathrm{E}+04$ \\
\hline Alkane Monooxygenase (ALK) & $<1.00 \mathrm{E}+04$ & $<1.00 \mathrm{E}+04$ \\
\hline Alkane Monooxygenase (ALMA) & $<1.00 \mathrm{E}+04$ & $<1.00 \mathrm{E}+04$ \\
\hline Anaerobic BTEX & - & - \\
\hline Benzoyl Coenzyme A Reductase (BCR) & $8.20 \mathrm{E}+08$ & $7.06 \mathrm{E}+07$ \\
\hline Benzylsuccinate synthase (BSS) & $<1.00 \mathrm{E}+04$ & $<1.00 \mathrm{E}+04$ \\
\hline Benzene Carboxylase (ABC) & $<1.00 \mathrm{E}+04$ & $<1.00 \mathrm{E}+04$ \\
\hline Anaerobic PAHs and Alkanes & - & - \\
\hline Naphthylmethylsuccinate Synthase (MNSSA) & $<1.00 \mathrm{E}+04$ & $<1.00 \mathrm{E}+04$ \\
\hline Naphthalene Carboxylase (ANC) & $<1.00 \mathrm{E}+04$ & $<1.00 \mathrm{E}+04$ \\
\hline Alklysuccinate Synthase (ASSA) & $<1.00 \mathrm{E}+04$ & $<1.00 \mathrm{E}+04$ \\
\hline Other & - & - \\
\hline Dehalococcoides spp. (DHC) & $2.34 \mathrm{E}+04$ & $<1.00 \mathrm{E}+03$ \\
\hline Total Eubacteria (EBAC) & $1.32 \mathrm{E}+09$ & $9.19 \mathrm{E}+07$ \\
\hline Sulfate Reducing Bacteria (APS) & $<1.00 \mathrm{E}+04$ & $<1.00 \mathrm{E}+04$ \\
\hline
\end{tabular}

$<=$ Not detected 


\subsection{0 qPCR Analysis}

qPCR revealed several important things about the microbial community in the soil at this site. First, a significant population of microbes was found in both soil samples tested that are known to aerobically degrade petroleum hydrocarbons. The genes for aerobically breaking down toluene, biphenyl and phenol were detected in both samples. Both samples also contained an indicator for Methylibium petroleiphilum PM1, which is one of the few isolated organisms that can use MTBE and TBA as growth-supporting substrates. Sample 2 also contained TBA monooxygenase, which is a gene utilized by Methylibium petroleiphilum PM1 to degrade TBA. The TRFLP pattern of Methylibium petroleiphilum PM1 was searched for in the fragment data, but fragment length created using the DpnII enzyme was too small (15 base pairs) to show up on the chromatographs. The presence of hydrocarbon degraders was expected because PHCs degraders are the most common out of all the COI biodegraders for several reasons. First, PHCs and BTEX biodegradation or more studied than that of the other COIs because they are more common contaminants. This means there is a better understanding of PHC biodegraders and pathways in comparison. Second, many constituents of TPH are easier to degrade than the other COIs (particularly PCBs and dioxin). Because of this, a population of PHC biodegraders is far more likely to out compete other bacteria than, for example, a population of dioxin degraders. Finally, because many constituents in TPH are easier to biodegrade, there are more bacteria with the genes necessary to do it. This increases the likelihood that a bacterium on the site has the ability to biodegrade PHCs.

The only target associated with anaerobic BTEX degradation that was detected was the Benzoyl Coenzyme A reductase (BCR) gene. This gene was present in both soil samples 
and is used in the breakdown of Benzoyl Coenzyme A, which is a common intermediate that is formed in many pathways for anaerobic biodegradation of aromatic hydrocarbons. It is unlikely that the BCR gene would be abundant in a predominantly aerobic environment, such as SSFL soil. However, the qPCR showed a high percentage (62 and $77 \%$ ) of the total population had this gene. It is probable that this result is inaccurate, and that these high frequencies are caused by the gene target used for BCR in the qPCR procedure not being specific enough to the BCR gene and reading false positives. Additionally, the fact that BCR is the only anaerobic gene target in either sample supports the theory of false positives. It is possible that PHCs is being biodegraded anaerobically on the site, although anaerobic metabolism would be expected to be very slow and only occur in anaerobic micro regions.

No targets for aerobic or anaerobic biodegradation of PAHs were observed in either soil sample. These results suggest that known microbes of natural attenuation of PAHs are not present in large populations at the site. It is possible that the more bioavailable or smaller PAHs have already been biodegraded leaving only recalcitrant PAHs (Cerniglia 1992b; Alexander 1995). These recalcitrant molecules are likely to be large or sequestered into the soil, making them difficult to biodegrade. Based on these qPCR results it is unlikely that PAHs are currently being biodegraded by bacteria in significant quantities on the site.

Dehalococcoides was found in the fresh soil sample (sample 1), which was the least handled and freshest sample. It is possible that there were originally Dehalococcoides in the stored composite sample (sample 2) as well but they may have died during the sieving process or storage. Although they are present, they need to be in an anaerobic 
environment to dechlorinate PCBs. Because all of the soil on the site is aerobic, it is unlikely that there is significant dechlorination PCBs or chlorinated dioxins. However, dechlorination is possible in anaerobic microenvironments in the soil. The microenvironments could exist in clay soils or in soil saturated with water. Biphenyl dioxygenase was also found in the fresh soil sample. This means that some of the microbes in this sample have the ability to breakdown the biphenyl backbone of a PCB molecule. Because both Dehalococcoides and biphenyl dioxygenase were found in Sample 1, it is conceivable that microbes in that community are dechlorinating PCBs in anaerobic microenvironments and then breaking down the resulting biphenyl backbone. This would mean that complete mineralization of PCBs is theoretically possible in Sample 1. The presence of Dehalococcoides in sample 1 also means that dechlorination of dioxins could be occurring in microenvironments in the soil. However, the population of Dehalococcoides was barely above the detection limit of the qPCR assay. Based on qPCR it is unlikely that PCBs or dioxins are being dechlorinated by Dehalococcoides species in significant quantities on the site. 


\subsection{Conclusions}

Based on both molecular biology methods and culturing experiments there is evidence of populations of bacteria and fungi in the SSFL soils which are capable of biodegrading the COIs. This evidence was seen in the results of both TRFLP and qPCR.

The culturing experiments isolated microbes growing in the soil of the site with the capacity to biodegrade model chemicals similar to the COIs. Ten different bacteria and 5 different fungi were isolated which are known biodegraders of the COIs or belong to genera that contain biodegraders. Most notable from this group is the 6 different strains of species of Pseudomonas, 2 species of Aspergillus, and 3 strains of Phanerochaete. The Pseudomonas genus has many species that are known degraders of PHCs (Tyagi, da Fonseca, and de Carvalho 2011b), PAHs (Haritash and Kaushik 2009), PCBs (Yong-lei et al. 2011), and chlorinated dioxins (Nam et al. 2006). Although none of the particular Pseudomonas strains isolated in this study have specifically been shown in the literature to biodegrade the COIs, it is possible that they have this capability and have not been studied yet. One of the strains of the fungi Aspergillus (Aspergillus fumigatus) has been shown by Tigini et al. (2009) to have the capability to biodegrade PCBs with the addition of glucose. Possibly the most important organism isolated from the soil samples was the fungi $P$. chrysosporium. Two strains of this fungal species were cultured from the soil as well as another member of the Phanerochaete genus. Phanerochaete chrysosporium is important because it has been shown to biodegrade all the COIs (Yateem et al. 1998; Novotný et al. 2004; Bumpus 1989; J. Bumpus et al. 1985). This fungi was isolated twice in 168 individual culturing plates that targeted fungal degraders. Since the frequency of 
this organism is so low it may not significantly contribute to the natural attenuation of the site, unless bioaugmentation is employed.

It is important to remember that the culturing was conducted under idealized lab conditions with model chemicals. In the soil, conditions are likely to be different and there are many carbon sources and many bacteria that may be able to out-compete the biodegrading microbes. To determine actual biodegradation rates of the COIs in SSFL soils, a companion study is currently underway using laboratory microcosms to measure biodegradation in actual site soils under simulated field conditions. Interactions between bacteria, fungi, and plants, which are also not considered in these experiments, are likely to have effects on microbial populations. Another companion study is currently underway to test for enhanced biodegradation in the presence of native plants from the site. A metagenomics study is also underway which may shed more light on the microbial communities in SSFL soils.

The TRFLP analysis of the 30 soil samples did not show a significant correlation of microbial populations with the presence of any of the COIs. This indicates that the bacteria and/or fungi which degrade the COIs are either present in small numbers compared with other species, or they are broadly distributed across the site. However, the TRFLP peaks of all 6 positive control organisms (including P. chrysosporium) were found in the fragment data. This indicates a strong probability that these known degraders (which collectively can biodegrade all the COIs) are in the soil.

There is strong evidence in the qPCR analysis showing that petroleum compounds are being aerobically biodegraded in the soil. The qPCR analysis showed the presence of 
bacteria/genes known to be involved in the aerobic biodegradation of PHCs. In contrast, the results of the qPCR and soil vapor analyses indicate that anaerobic activity, including anaerobic dechlorination, is very limited on this site. Because anaerobic reductive dechlorination is an essential step for bacterial biodegradation of PCBs and chlorinated dioxins this makes the natural attenuation of these COIs unlikely by bacteria. However, there was a small population of Dehalococcoides detected by qPCR in the fresh soil sample. This suggests that small anaerobic microenvironments exist in the soil where these microbes may be able to carry out anaerobic dechlorination. However, the small amount of these microbes is not likely large enough to rely on for the natural attenuation of these chlorinated compounds

The only anaerobic target gene detected was benzoyl coenzyme A reductase, which was present in both soil samples. Benzoyl coenzyme A reductase (BCR) is an enzyme that attacks a major intermediate product of the anaerobic biodegradation pathways of various hydrocarbons. It is unlikely that the BCR gene would be abundant in a predominantly aerobic environment, such as SSFL soil. However, the qPCR showed a high percentage (62\% and $77 \%)$ of the total population had this gene. These unlikely results may be caused by the primers used for the BCR assay not being specific enough to the BCR gene. This would result in false positives. Additionally, the fact that BCR is the only anaerobic gene target in either sample supports the theory of false positives. There was no evidence of aerobic PAH degrading genes being present in the soil. It is possible that in the past these processes were occurring but over the last few decades the bioavailable PAHs have been biodegraded leaving only the larger more recalcitrant ones. Also, weathering is likely to have occurred over this time making the remaining PAHs less 
bioavailable. The qPCR analysis only covered bacterial species and gene targets, and thus can provide no insight to possible fungal species and gene targets. It is possible that in areas with clay or high moisture the soil may contain anaerobic regions. It is also possible that these anaerobic regions may form due to precipitation. However, these regions are unlikely to have a large effect on the concentrations of the COIs in the bulk soil. Since fungi do not require anaerobic conditions to biodegrade the COIs, the fungal pathway may be more promising than a bacterial pathway.

Collectively these experiments showed that there are bacteria and fungi on the site which can biodegrade each of the COIs. However, successful natural attenuation at this site will require sufficient microbial populations and conditions which are conducive to biodegradation. Further research is underway in one of the companion studies to determine if biostimulation or bioaugmentation could be used to remediate this site. The most practical way of doing this would be to add nutrients, surfactants, and/or bulking agents that would support the growth of bacteria or fungi. Phanerochaete chrysosporium is the most promising species for bioaugmentation because it can degrade all COIs completely using its non substrate-specific enzymes. A lignin-rich substrate, such as wood chips, would need to be added to induce the production of the non-substrate specific enzymes that can degrade these COIs. As stated above, this fungus also does not require anaerobic conditions as bacterial dechlorination does and is thus the most promising. The bioremediation companion study is testing for the efficacy of bioaugmentation using Phanerochaete chrysosporium. Since this fungus was isolated from soils at SSFL, it is likely to survive well in SSFL soils. 


\section{REFERENCES}

Abalos, A., M. Viñas, J. Sabaté, M. A. Manresa, and A. M. Solanas. 2004. "Enhanced Biodegradation of Casablanca Crude Oil by A Microbial Consortium in Presence of a Rhamnolipid Produced by Pseudomonas Aeruginosa AT10." Biodegradation 15 (4): 249-60. doi:10.1023/B:BIOD.0000042915.28757.fb.

Abraham, Wolf-Rainer, Balbina Nogales, Peter N Golyshin, Dietmar H Pieper, and Kenneth N Timmis. 2002. "Polychlorinated Biphenyl-Degrading Microbial Communities in Soils and Sediments." Current Opinion in Microbiology 5 (3): 246-53. doi:10.1016/S1369-5274(02)00323-5.

acumedia. 2010a. "Tryptic Soy Broth (7164)." http://www.neogen.com/Acumedia/pdf/ProdInfo/7164_PI.pdf.

. 2010b. "YM Broth (7363)." http://www.neogen.com/Acumedia/pdf/ProdInfo/7363_PI.pdf.

Alexander, Martin. 1995. "How Toxic Are Toxic Chemicals in Soil?" Environmental Science \& Technology 29 (11): 2713-17. doi:10.1021/es00011a003.

Alvarez-Bernal, D., E. L. Garcia-Diaz, S. M. Contreras-Ramos, and L. Dendooven. 2006. "Dissipation of Polycyclic Aromatic Hydrocarbons from Soil Added with Manure or Vermicompost." Chemosphere 65 (9): 1642-51. doi:10.1016/j.chemosphere.2006.02.028.

Arfmann, H, K N Timmis, and R Wittich. 1997. "Mineralization of 4Chlorodibenzofuran by a Consortium Consisting of Sphingomonas Sp. Strain RW1 and Burkholderia Sp. Strain JWS." Applied and Environmental Microbiology 63 (9): 3458-62.

ASTDR. “ATSDR - Toxprofile: Toxicological Profile Information Sheet: PCBs.”

_. 2010. “ATSDR - Toxprofile: Toxicological Profile Information Sheet: PCBs.” http://www.atsdr.cdc.gov/toxprofiles/tp17-c4.pdf.

Atlas, R M. 1981. "Microbial Degradation of Petroleum Hydrocarbons: An Environmental Perspective.” Microbiological Reviews 45 (1): 180-209.

ATSDR. 2009. “Agency for Toxic Substances and Disease Registry (ATSDR) Case Studies in Environmental Medicine Toxicity of Polycyclic Aromatic Hydrocarbons (PAHs)."

“ATSDR - Toxic Substances - Total Petroleum Hydrocarbons (TPH).”

Ballerstedt, Hendrik, Janina Hantke, Michael Bunge, Birgit Werner, Jan Gerritse, Jan R Andreesen, and Ute Lechner. 2004. "Properties of a Trichlorodibenzo-P-DioxinDechlorinating Mixed Culture with a Dehalococcoides as Putative Dechlorinating 
Species.” FEMS Microbiology Ecology 47 (2): 223-34. doi:10.1016/S01686496(03)00282-4.

Bedard, Donna L., and Marie L. Haberl. 1990. "Influence of Chlorine Substitution Pattern on the Degradation of Polychlorinated Biphenyls by Eight Bacterial Strains.” Microbial Ecology 20 (2): 87-102. doi:10.2307/4251142.

Bedard, Donna L., Kirsti M. Ritalahti, and Frank E. Löffler. 2007. “The Dehalococcoides Population in Sediment-Free Mixed Cultures Metabolically Dechlorinates the Commercial Polychlorinated Biphenyl Mixture Aroclor 1260." Applied and Environmental Microbiology 73 (8): 2513-21. doi:10.1128/AEM.02909-06.

Beilen, Jan B van, and Enrico G Funhoff. 2005. "Expanding the Alkane Oxygenase Toolbox: New Enzymes and Applications." Current Opinion in Biotechnology 16 (3): 308-14. doi:10.1016/j.copbio.2005.04.005.

Bento, Fatima, Flavio Camargo, Benedict Okeke, and William Frankenberger. 2005. "Comparative Bioremediation of Soils Contaminated with Diesel Oil by Natural Attenuation, Biostimulation and Bioaugmentation." Science Direct 96: 1049-55. doi:10.1016/j.biortech.2004.09.008.

Bergendahl, John. 2005. "Batch Leaching Tests: Colloid Release and PAH Leachability." Soil \& Sediment Contamination 14 (6): 527-43.

doi:10.1080/15320380500263725.

Bernard, Alfred, Fabrice Broeckaert, Geert De Poorter, Ann De Cock, Cédric Hermans, Claude Saegerman, and Gilbert Houins. 2002. "The Belgian PCB/Dioxin Incident: Analysis of the Food Chain Contamination and Health Risk Evaluation." Environmental Research 88 (1): 1-18. doi:10.1006/enrs.2001.4274.

Beurskens, Jacobus E.M., Johan De Wolf, Erel Toussaint, Jan M.D. van der Steen, Pieter C. Slot, Laetitia C.M. Commandeur, and John R. Parsons. 1995. "Dehalogenation of Chlorinated Dioxins by an Anaerobic Microbial Consortium from Sediment." Environmental Toxicology and Chemistry 14 (6): 939-43. doi:10.1002/etc.5620140603.

Beyer, Angelika, and Marek Biziuk. 2009. "Environmental Fate and Global Distribution of Polychlorinated Biphenyls." Reviews of Environmental Contamination and Toxicology 201: 137-58. doi:10.1007/978-1-4419-0032-6_5.

"Boeing: Santa Susana.” 2014a. Santa Susana Homepage. Accessed July 8. http://www.boeing.com/boeing/aboutus/environment/santa_susana/index.page. . 2014b. Santa Susana History. Accessed July 8. http://www.boeing.com/boeing/aboutus/environment/santa_susana/history.page. 
Boening, D. W. 1998. "Toxicity of 2,3,7,8-Tetrachlorodibenzo-P-Dioxin to Several Ecological Receptor Groups: A Short Review.” Ecotoxicology and Environmental Safety 39 (3): 155-63. doi:10.1006/eesa.1997.1608.

Borg, Ingwer, and Patrick J. F. Groenen. 1997. Modern Multidimensional Scaling: Theory and Applications : With 116 Figures. Springer-Verlag GmbH.

Borja, Josephine, Donna Marie Taleon, Joseph Auresenia, and Susan Gallardo. 2005. "Polychlorinated Biphenyls and Their Biodegradation." Process Biochemistry 40 (6): 1999-2013. doi:10.1016/j.procbio.2004.08.006.

Brooijmans, Rob J W, Margreet I Pastink, and Roland J Siezen. 2009. "HydrocarbonDegrading Bacteria: The Oil-Spill Clean-up Crew." Microbial Biotechnology 2 (6): 587-94. doi:10.1111/j.1751-7915.2009.00151.x.

Brühlmann, Fredi, and Wilfred Chen. 1999. "Transformation of Polychlorinated Biphenyls by a Novel BphA Variant through the Meta-Cleavage Pathway." FEMS Microbiology Letters 179 (2): 203-8. doi:10.1111/j.1574-6968.1999.tb08728.x.

Brzuzy, Louis P., and Ronald A. Hites. 1996. "Global Mass Balance for Polychlorinated Dibenzo- P -Dioxins and Dibenzofurans." Environmental Science \& Technology 30 (6). American Chemical Society: 1797-1804. doi:10.1021/es950714n.

Bumpus, J. A. 1989. "Biodegradation of Polycyclic Hydrocarbons by Phanerochaete Chrysosporium." Applied and Environmental Microbiology 55 (1): 154-58.

Bumpus, Ja, M. Tien, D. Wright, and Sd Aust. 1985. "Oxidation of Persistent Environmental-Pollutants by a White Rot Fungus.” Science 228 (4706): 1434-36. doi:10.1126/science.3925550.

Bunge, Michael, Lorenz Adrian, Angelika Kraus, Matthias Opel, Wilhelm G Lorenz, Jan R Andreesen, Helmut Görisch, and Ute Lechner. 2003. "Reductive Dehalogenation of Chlorinated Dioxins by an Anaerobic Bacterium." Nature 421 (6921): 357-60. doi:10.1038/nature01237.

Bunge, Michael, and Ute Lechner. 2009. "Anaerobic Reductive Dehalogenation of Polychlorinated Dioxins.” Applied Microbiology and Biotechnology 84 (3): 42944. doi:10.1007/s00253-009-2084-7.

Bunge, Michael, Anke Wagner, Marco Fischer, Jan R Andreesen, and Ute Lechner. 2008. "Enrichment of a Dioxin-Dehalogenating Dehalococcoides Species in TwoLiquid Phase Cultures." Environmental Microbiology 10 (10): 2670-83. doi:10.1111/j.1462-2920.2008.01688.x.

California Energy Comission. 2012. "Nuclear Energy in California - California Energy Commission." California Energy Comission. http://www.energy.ca.gov/nuclear/california.html. 
CDM Smith. 2013a. "Clearly Contaminated Areas Summary."

—. 2013b. "Composite Interpolated Soil Cehmicals by Group (map)."

Cerniglia, Carl E. 1992a. "Biodegradation of Polycyclic Aromatic Hydrocarbons." Biodegradation 3 (2-3): 351-68. doi:10.1007/BF00129093.

1992b. "Biodegradation of Polycyclic Aromatic Hydrocarbons." Biodegradation 3 (2-3): 351-68. doi:10.1007/BF00129093.

Chell, M., L. Rastagarzadeh, and Y. M. Nelson. 2007. "Diluent Hydrocarbon Biodegradation in Laboratory Microcosms Using Anoxic Electron Acceptors." Presented at the 9th International Conference on in-Situ and on-Site Bioremediation." In 9th International Conference on In-Situ and on-Site Bioremediation.

"ChemSpider." 2013. Database. ChemSpider: The Free Chemical Database. http://www.chemspider.com/.

Chew, Melton. 2006. "Energy Technology Engineering Center - Site Description”. Oak Ridge Associated Universities, Dade Moeller \& Associates, MJW Corporation. http://www.cdc.gov/niosh/ocas/pdfs/tbd/etec2.pdf.

Complete Genomics. 2013. "Complete Genomics >> Extracting and Storing DNA." Complete Genomics Incorporated. http://www.completegenomics.com/FAQs/Extracting-and-Storing-DNA/\#q5.

Consonni, Dario, Angela C Pesatori, Carlo Zocchetti, Raffaella Sindaco, Luca Cavalieri D'Oro, Maurizia Rubagotti, and Pier Alberto Bertazzi. 2008. "Mortality in a Population Exposed to Dioxin after the Seveso, Italy, Accident in 1976: 25 Years of Follow-Up.” American Journal of Epidemiology 167 (7): 847-58. doi:10.1093/aje/kwm371.

Cornelissen, Gerard, Örjan Gustafsson, Thomas D. Bucheli, Michiel T. O. Jonker, Albert A. Koelmans, and Paul C. M. van Noort. 2005. "Extensive Sorption of Organic Compounds to Black Carbon, Coal, and Kerogen in Sediments and Soils: Mechanisms and Consequences for Distribution, Bioaccumulation, and Biodegradation." Environmental Science \& Technology 39 (18). American Chemical Society: 6881-95. doi:10.1021/es050191b.

Čvančarová, Monika, Zdena Křesinová, Alena Filipová, Stefano Covino, and Tomáš Cajthaml. 2012. "Biodegradation of PCBs by Ligninolytic Fungi and Characterization of the Degradation Products." Chemosphere 88 (11): 1317-23. doi:10.1016/j.chemosphere.2012.03.107.

Dados, Angelos, Michalis Omirou, Kyproula Demetriou, Chara Papastephanou, and Ioannis M. Ioannides. 2014. "Rapid Remediation of Soil Heavily Contaminated 
with Hydrocarbons: A Comparison of Different Approaches." Annals of Microbiology, March, 1-11. doi:10.1007/s13213-014-0856-5.

Das, Nilanjana, and Preethy Chandran. 2011. "Microbial Degradation of Petroleum Hydrocarbon Contaminants: An Overview." Biotechnology Research International 2011 (January): 941810. doi:10.4061/2011/941810.

Davis, Brian, and Michael Wade. 2003. "RISK ASSESSMENT OF POLYCHLORINATED BIPHENYLS AT HAZARDOUS WASTE SITES". DTSC.

Dubey, George A., and Hardev S. Dugal. 1977. "Polychlorinated Biphenyls in Pulp and Paper Mills. Part 2, Distribution and Removal. Project 3295, Report Two : A Progress Report to Members of the Institute of Paper Chemistry". Project Report.

Eaton, David C. 1985. "Mineralization of Polychlorinated Biphenyls by Phanerochaete Chrysosporium: A Ligninolytic Fungus." Enzyme and Microbial Technology 7 (5): 194-96. doi:10.1016/S0141-0229(85)80001-6.

EPA. 2013. "Priority Pollutants." Appendix A to 40 CFR Part 423, Priority Pollutants.

Espinoza, Rivera, and L Dendooven. 2007. "Dynamics of Carbon and Nitrogen in a Mixture of Polycyclic Aromatic Hydrocarbons Contaminated Soil Amended with Organic Residues." Environmental Technology 28 (8): 883-93.

ETEC. 2005a. "TopangaWildfirePosterPictures.pdf”. ETEC. http://www.etec.energy.gov/Library/Main/TopangaWildfirePosterPictures.pdf.

—. 2005b. "TopangaWildfirePosterPictures.pdf". ETEC.

Fennell, Donna E., Ivonne Nijenhuis, Susan F. Wilson, Stephen H. Zinder, and Max M. Häggblom. 2004. "Dehalococcoides Ethenogenes Strain 195 Reductively Dechlorinates Diverse Chlorinated Aromatic Pollutants." Environmental Science \& Technology 38 (7): 2075-81. doi:10.1021/es034989b.

Fernández-Sánchez, J. M., R. Rodríguez-Vázquez, G. Ruiz-Aguilar, and P. J. J. Alvarez. 2001. "Pcb Biodegradation in Aged Contaminated Soil: Interactions Between Exogenous Phanerochaete Chrysosporium and Indigenous Microorganisms." Journal of Environmental Science and Health, Part A 36 (7): 1145-62. doi:10.1081/ESE-100104869.

Fiedler, Heidelore. 1997. "Polychlorinated Biphenyls (PCBs): Uses and Environmental Releases." In Polychlorinated Biphenyls (PCBs): Uses and Environmental Releases. Bangkok, Thailand: United Nations Environment Programme.

Field, Jim A, and Reyes Sierra-Alvarez. 2008. "Microbial Degradation of Chlorinated Dioxins." Chemosphere 71 (6): 1005-18. doi:10.1016/j.chemosphere.2007.10.039. 
Furukawa, K., N. Tomizuka, and A. Kamibayashi. 1979. "Effect of Chlorine Substitution on the Bacterial Metabolism of Various Polychlorinated Biphenyls." Applied and Environmental Microbiology 38 (2): 301-10.

Gallego, José R., Carlos Sierra, Ramón Villa, Ana I. Peláez, and Jesús Sánchez. 2010. "Weathering Processes Only Partially Limit the Potential for Bioremediation of Hydrocarbon-Contaminated Soils." Organic Geochemistry 41 (9): 896-900. doi:10.1016/j.orggeochem.2010.04.021.

Gibson, D. T., J. R. Koch, and R. E. Kallio. 1968. "Oxidative Degradation of Aromatic Hydrocarbons by Microorganisms. I. Enzymic Formation of Catechol from Benzene.” Biochemistry 7 (7): 2653-62. doi:10.1021/bi00847a031.

Gibson, D. T., G. J. Zylstra, and S. Chauhan. 1990. "Biotransformations Catalyzed by Toluene Dioxygenase from Pseudomonas Putida F 1."

Gibson, David T. 1984. "Microbial Degradation of Organic Compounds.”

Gieg, L. M., R. V. Kolhatkar, M. J. McInerney, R. S. Tanner, S. H. Harris, K. L. Sublette, and J. M. Suflita. 1999. "Intrinsic Bioremediation of Petroleum Hydrocarbons in a Gas Condensate-Contaminate Aquifer.” Environmental Science \& Technology 33 (15): 2550-60. doi:10.1021/es981349d.

Grishchenkov, V.G., R.T. Townsend, T.J. McDonald, R.L. Autenrieth, J.S. Bonner, and A.M. Boronin. 2000. "Degradation of Petroleum Hydrocarbons by Facultative Anaerobic Bacteria under Aerobic and Anaerobic Conditions." Process Biochemistry 35 (9): 889-96.

Haemmerli, S D, M S Leisola, D Sanglard, and A Fiechter. 1986. "Oxidation of Benzo(a)pyrene by Extracellular Ligninases of Phanerochaete Chrysosporium. Veratryl Alcohol and Stability of Ligninase." The Journal of Biological Chemistry 261 (15): 6900-6903.

Haggblom, Max, Donna Fennell, Lisa Rodenburg, Lee Kerkhof, and Kevin Sowers. 2012. "SERDP Project ER-1492."

Haglund, Peter. 2007. "Methods for Treating Soils Contaminated with Polychlorinated Dibenzo-P-Dioxins, Dibenzofurans, and Other Polychlorinated Aromatic Compounds - ProQuest." AMBIO: A Journal of the Human Environment. http://www.bioone.org/doi/abs/10.1579/00447447(2007)36[467:MFTSCW]2.0.CO;2.

Hammel, K. E., W. Z. Gai, B. Green, and M. A. Moen. 1992. "Oxidative Degradation of Phenanthrene by the Ligninolytic Fungus Phanerochaete Chrysosporium." Applied and Environmental Microbiology 58 (6): 1832-38. 
Hammel, K. E., B. Kalyanaraman, and T. K. Kirk. 1986. "Oxidation of Polycyclic Aromatic Hydrocarbons and Dibenzo[p]-Dioxins by Phanerochaete Chrysosporium Ligninase.” Journal of Biological Chemistry 261 (36): 16948-52.

Hanson, Jessica R., Corinne E. Ackerman, and Kate M. Scow. 1999. "Biodegradation of Methyl Tert-Butyl Ether by a Bacterial Pure Culture." Applied and Environmental Microbiology 65 (11): 4788-92.

Haritash, a K, and C P Kaushik. 2009. "Biodegradation Aspects of Polycyclic Aromatic Hydrocarbons (PAHs): A Review." Journal of Hazardous Materials 169 (1-3): 115. doi:10.1016/j.jhazmat.2009.03.137.

Hatzinger, P B, and M Alexander. 1995. "Effect of Aging of Chemicals in Soil on Their Biodegradability and Extractability." Environmental Science \& Technology 29 (2). American Chemical Society: 537-45. doi:10.1021/es00002a033.

Holliger, C, G Schraa, A J Stams, and A J Zehnder. 1992. "Enrichment and Properties of an Anaerobic Mixed Culture Reductively Dechlorinating 1,2,3-Trichlorobenzene to 1,3-Dichlorobenzene." Applied and Environmental Microbiology 58 (5): 163644.

Hong, Hyo-Bong, Yoon-Seok Chang, In-Hyun Nam, Peter Fortnagel, and Stefan Schmidt. 2002. "Biotransformation of 2,7-Dichloro- and 1,2,3,4Tetrachlorodibenzo-P-Dioxin by Sphingomonas Wittichii RW1.” Applied and Environmental Microbiology 68 (5): 2584-88. doi:10.1128/AEM.68.5.25842588.2002.

Hong, Hyo-Bong, In-Hyun Nam, Kumarasamy Murugesan, Young-Mo Kim, and YoonSeok Chang. 2004. "Biodegradation of Dibenzo-P-Dioxin, Dibenzofuran, and Chlorodibenzo-P-Dioxins by Pseudomonas Veronii PH-03." Biodegradation 15 (5): 303-13.

Hyun, Seunghun, Minhee Kim, Kitae Baek, and Linda S. Lee. 2010. "Phenanthrene and 2,2',5,5'-PCB Sorption by Several Soils from Methanol-water Solutions: The Effect of Weathering and Solute Structure." Chemosphere 78 (4): 423-29. doi:10.1016/j.chemosphere.2009.10.055.

Isensee, Allan R., and Gerald E. Jones. 1975. "Distribution of 2,3,7,8Tetrachlorodibenzo-P-Dioxin (TCDD) in Aquatic Model Ecosystem." Environmental Science \& Technology 9 (7). American Chemical Society: 668-72. doi:10.1021/es60105a007.

Ishii, Kazuei, Toru Furuichi, Noboru Tanikawa, and Masaru Kuboshima. 2009.

"Estimation of the Biodegradation Rate of 2,3,7,8-Tetrachlorodibenzo-P-Dioxin by Using Dioxin-Degrading Fungus, Pseudallescheria Boydii." Journal of Hazardous Materials 162 (1): 328-32. doi:10.1016/j.jhazmat.2008.05.043. 
Isosaan, P , Tuhkanen, T and Vartiainen, T 2001. 2004. "Photodegradation of Polychlorinated Dibenzo-P-Dioxins and Dibenzofurans in Soil with Vegetable Oil - ProQuest." Environ SciPollut Res 11: 181-85.

Jacques, Rodrigo J S, Benedict C Okeke, Fátima M Bento, Maria C R Peralba, and Flávio a O Camargo. 2009. "Improved Enrichment and Isolation of Polycyclic Aromatic Hydrocarbons (PAH)-Degrading Microorganisms in Soil Using Anthracene as a Model PAH." Current Microbiology 58 (6): 628-34. doi:10.1007/s00284-0099381-3.

Jones, Jack, Rochelle Arujo, and John Rodgers. 2012. "Bench-Scale Evaluation of Bioremediation for the Treatment of Sediments from the Ashtabula, Buffalo, Saginaw and Sheboygan Rivers." US EPA. http://www.epa.gov/glnpo/arcs/r96012/r96012.html.

Joshi, Dinesh K., and Michael H. Gold. 1994. "Oxidation of Dibenzo-P-Dioxin by Lignin Peroxidase from the Basidiomycete Phanerochaete Chrysosporium." Biochemistry 33 (36): 10969-76. doi:10.1021/bi00202a016.

Juteau, P., D. Rho, R. Larocque, and A. LeDuy. 1999. "Analysis of the Relative Abundance of Different Types of Bacteria Capable of Toluene Degradation in a Compost Biofilter." Applied Microbiology and Biotechnology 52 (6): 863-68. doi:10.1007/s002530051604.

Kamei, Ichiro, Hiroto Suhara, and Ryuichiro Kondo. 2005. "Phylogenetical Approach to Isolation of White-Rot Fungi Capable of Degrading Polychlorinated Dibenzo-PDioxin." Applied Microbiology and Biotechnology 69 (3): 358-66. doi:10.1007/s00253-005-0052-4.

Kamei, Ichiro, Masashi Watanabe, Kazuhisa Harada, Takashi Miyahara, Shinya Suzuki, Yasushi Matsufuji, and Ryuichiro Kondo. 2009. "Influence of Soil Properties on the Biodegradation of 1,3,6,8-Tetrachlorodibenzo-P-Dioxin and Fungal Treatment of Contaminated Paddy Soil by White Rot Fungus Phlebia Brevispora." Chemosphere 75 (10): 1294-1300. doi:10.1016/j.chemosphere.2009.03.028.

Kang, H K, K K Watanabe, J Breen, J Remmers, M G Conomos, J Stanley, and M Flicker. 1991. "Dioxins and Dibenzofurans in Adipose Tissue of US Vietnam Veterans and Controls." American Journal of Public Health 81 (3): 344-49.

Kaplan, Christopher W, and Christopher L Kitts. 2004. "Bacterial Succession in a Petroleum Land Treatment Unit." Applied and Environmental Microbiology 70 (3): 1777-86.

Kasai, Noriyuki, Shin-Ichi Ikushiro, Raku Shinkyo, Kaori Yasuda, Shinji Hirosue, Akira Arisawa, Hirofumi Ichinose, Hiroyuki Wariishi, and Toshiyuki Sakaki. 2010. "Metabolism of Mono- and Dichloro-Dibenzo-P-Dioxins by Phanerochaete Chrysosporium Cytochromes P450." Applied Microbiology and Biotechnology, January, 773-80. doi:10.1007/s00253-009-2413-x. 
Kearney, Pc, Ea Woolson, and Cp Ellingto. 1972. "Persistence and Metabolism of Chlorodioxins in Soils." Environmental Science \& Technology 6 (12): 1017-\&. doi:10.1021/es60071a010.

Kieatiwong, Somchai, Long V. Nguyen, Vincent R. Hebert, Murray Hackett, Glenn C. Miller, Michael J. Miille, and Robert Mitzel. 1990. "Photolysis of Chlorinated Dioxins in Organic Solvents and on Soils." Environmental Science \& Technology 24 (10): 1575-80. doi:10.1021/es00080a018.

Kjeller, Lars-Owe., and Christoffer. Rappe. 1995. "Time Trends in Levels, Patterns, and Profiles for Polychlorinated Dibenzo-P-Dioxins, Dibenzofurans, and Biphenyls in a Sediment Core from the Baltic Proper." Environmental Science \& Technology 29 (2): 346-55. doi:10.1021/es00002a010.

Kuroda, Haruo. 1964. "Ionization Potentials of Polycyclic Aromatic Hydrocarbons." Nature 201 (4925): 1214-15. doi:10.1038/2011214a0.

Kyser, Scott, Raymond Hozalski, and John Gulliver. 2011. "Use of Compost to Biodegrade Sediments Contaminated with Polycyclic Aromatic Hydrocarbons". presented at the University of Minnesota Stormwater Assessment Project, St. Anthony Falls Laboratory University of Minnesota, March 21. http://www.pca.state.mn.us/index.php/view-document.html?gid=18449.

Laskin, Allen I. 1984. ADVANCES IN APPLIED MICROBIOLOGY. Academic Press.

Leahy, Jg, and Rr Colwell. 1990. "Microbial-Degradation of Hydrocarbons in the Environment." Microbiological Reviews 54 (3): 305-15.

Levine, Michale D. 2013. "Hydrocarbon Toxicity.” WebMD LLC, March.

Li, Li, Qinggang Li, Fuli Li, Quan Shi, Bo Yu, Fengrui Liu, and Ping Xu. 2006. "Degradation of Carbazole and Its Derivatives by a Pseudomonas Sp." Applied Microbiology and Biotechnology 73 (4): 941-48. doi:10.1007/s00253-006-05303.

Lindsey, A. S., J. J. Belliardo, and P. J. Wagstaffe. 1989. "Utilisation of Certified Reference Materials in Monitoring Common Environmental Pollutants (PAHs, Nitro-PAHs, PCBs).” Fresenius' Zeitschrift Für Analytische Chemie 333 (6): 599-606. doi:10.1007/BF00496930.

Lundstedt, Staffan. 2003. "Analysis of PAHs and Their Transformation Products in Contaminated Soil and Remedial Processes". Umeå University. http://www.divaportal.org/smash/get/diva2:143820/FULLTEXT01.pdf.

Margesin, R., D. Labbe, F. Schinner, C. W. Greer, and L. G. Whyte. 2003. "Characterization of Hydrocarbon-Degrading Microbial Populations in Contaminated and Pristine Alpine Soils." Applied and Environmental Microbiology 69 (6): 3085-92. doi:10.1128/AEM.69.6.3085-3092.2003. 
Marshall, Alan G., and Ryan P. Rodgers. 2008. "Petroleomics: Chemistry of the Underworld." Proceedings of the National Academy of Sciences of the United States of America 105 (47): 18090-95. doi:10.1073/pnas.0805069105.

Microbial Insights. 2012. "ER Overview | Overview.” Microbial Insights: Environmental Remediation. http://www.microbe.com/index.php/Overview/er-overview.html.

—. 2014a. "QuantArray®—Petroleum." http://www.microbe.com/images/stories/handouts/quantarray_petroleum.pdf.

- 2014b. "CENSUS® Overview." http://www.microbe.com/images/stories/handouts/census.pdf.

Miller, Glenn C., Vincent R. Hebert, Michael J. Mille, Robert Mitzel, and Richard G. Zepp. 1989. "Photolysis of Octachlorodibenzo-P-Dioxin on Soils: Production of 2,3,7,8-TCDD." Chemosphere 18 (1): 1265-74.

Ministry for the Environment, New Zealand. 1996. "Appendix 4E Leaching Modeling, Guidelines for Assessing and Managing Petroleum Hydrocarbon Contaminated Sites in New Zealand", 1-14.

Moldes, Ana Belén, Remigio Paradelo, David Rubinos, Rosa Devesa-Rey, José Manuel Cruz, and María Teresa Barral. 2011. "Ex Situ Treatment of HydrocarbonContaminated Soil Using Biosurfactants from Lactobacillus Pentosus." Journal of Agricultural and Food Chemistry 59 (17): 9443-47. doi:10.1021/jf201807r.

N. K. Nagpal. 1993. "Ambient Water Quality Criteria For Polycyclic Aromatic Hydrocarbons (PAHs)." Ambient Water Quality Criteria For Polycyclic Aromatic Hydrocarbons (PAHs). http://www.env.gov.bc.ca/wat/wq/BCguidelines/pahs/index.html\#TopOfPage.

Nakamiya, Kunichika, Syunji Hashimoto, Hiroyasu Ito, John S. Edmonds, and Masatoshi Morita. 2005. "Degradation of 1,4-Dioxane and Cyclic Ethers by an Isolated Fungus." Applied and Environmental Microbiology 71 (3): 1254-58. doi:10.1128/AEM.71.3.1254-1258.2005.

Nam, In-Hyun, Young-Mo Kim, Stefan Schmidt, and Yoon-Seok Chang. 2006. "Biotransformation of 1,2,3-Tri- and 1,2,3,4,7,8-Hexachlorodibenzo-P- Dioxin by Sphingomonas Wittichii Strain RW1." Applied and Environmental Microbiology 72 (1): 112-16. doi:10.1128/AEM.72.1.112-116.2006.

Namkoong, Wan, Eui-Young Hwang, Joon-Seok Park, and Jung-Young Choi. 2002. "Bioremediation of Diesel-Contaminated Soil with Composting." Environmental Pollution (Barking, Essex: 1987) 119 (1): 23-31.

Narquis, C. T. 2007. "Generating the Right Data: Determination of Aroclors Versus PCB Congeners". FLUOR. 
Nicholson, S. E., and T. J. Farrar. 1994. "The Influence of Soil Type on the Relationships between NDVI, Rainfall, and Soil Moisture in Semiarid Botswana. I. NDVI

Response to Rainfall." Remote Sensing of Environment 50 (2): 107-20. doi:10.1016/0034-4257(94)90038-8.

Nies, Loring, and Timothy M. Vogel. 1990. "Effects of Organic Substrates on Dechlorination of Aroclor 1242 in Anaerobic Sediments." Applied and Environmental Microbiology 56 (9): 2612-17.

Nishiwaki, Junko, Yoshishige Kawabe, Yasuhide Sakamoto, Takeshi Komai, and Ming Zhang. 2011. "Volatilization Properties of Gasoline Components in Soils." Environmental Earth Sciences 63 (1): 87-95. doi:10.1007/s12665-010-0671-7.

Nojiri, Hideaki, Hiroshi Habe, and Toshio Omori. 2001a. "Bacterial Degradation of Aromatic Compounds via Angular Dioxygenation." The Journal of General and Applied Microbiology 47 (6): 279-305.

_. 2001b. "Bacterial Degradation of Aromatic Compounds via Angular Dioxygenation." The Journal of General and Applied Microbiology 47 (6): 279305.

Novotný, Čeněk, Kateřina Svobodová, Pavla Erbanová, Tomáš Cajthaml, Aparna Kasinath, Elke Lang, and Václav Šašek. 2004. "Ligninolytic Fungi in Bioremediation: Extracellular Enzyme Production and Degradation Rate." Soil Biology and Biochemistry 36 (10): 1545-51. doi:10.1016/j.soilbio.2004.07.019.

Osuji, Leo C., Inimfon A. Udoetok, and Regina E. Ogali. 2006. "Attenuation of Petroleum Hydrocarbons by Weathering: A Case Study." Chemistry \& Biodiversity 3 (4): 422-33. doi:10.1002/cbdv.200690046.

Panteleyev, Andrey A, and David R Bickers. 2006. "Dioxin-Induced Chloracne-Reconstructing the Cellular and Molecular Mechanisms of a Classic Environmental Disease.” Experimental Dermatology 15 (9): 705-30. doi:10.1111/j.1600-0625.2006.00476.x.

Park, Kap S., Ronald C. Sims, R. Ryan Dupont, William J. Doucette, and John E. Matthews. 1990. "Fate of PAH Compounds in Two Soil Types: Influence of Volatilization, Abiotic Loss and Biological Activity." Environmental Toxicology and Chemistry 9 (2): 187-95. doi:10.1002/etc.5620090208.

Patel, T. R., and D. T. Gibson. 1974. "Purification and Propeties of (+)-Cis-Naphthalene Dihydrodiol Dehydrogenase of Pseudomonas Putida." Journal of Bacteriology 119 (3): 879-88.

Pelz, O., M. Tesar, R. M. Wittich, E. R. B. Moore, K. N. Timmis, and W. R. Abraham. 1999. "Towards Elucidation of Microbial Community Metabolic Pathways: Unravelling the Network of Carbon Sharing in a Pollutant-Degrading Bacterial Consortium by Immunocapture and Isotopic Ratio Mass Spectrometry." 
Environmental Microbiology 1 (2): 167-74. doi:10.1046/j.1462-

2920.1999.00023.x.

Peng, Peng, Haiyan Yang, Ruibao Jia, and Li Li. 2013. "Biodegradation of Dioxin by a Newly Isolated Rhodococcus Sp. with the Involvement of Self-Transmissible Plasmids." Applied Microbiology and Biotechnology 97 (12): 5585-95. doi:10.1007/s00253-012-4363-y.

Pérez-Armendáriz, Beatriz, Daniel Martínez-Carrera, María Calixto-Mosqueda, Joel Alba, and Refugio Rodríguez-Vázquez. 2012. "Filamentous Fungi Remove Weathered Hydrocarbons from Polluted Soil of Tropical Mexico", July. http://repositoriodigital.academica.mx/jspui/handle/987654321/85199.

Pieper, Dietmar H. 2004. "Aerobic Degradation of Polychlorinated Biphenyls." Applied Microbiology and Biotechnology 67 (2): 170-91. doi:10.1007/s00253-004-18104.

Pierzynski, Gary M., George F. Vance, and J. Thomas Sims. 2005. Soils and Environmental Quality. CRC Press.

Pointing, S B. 2001. "Feasibility of Bioremediation by White-Rot Fungi." Applied Microbiology and Biotechnology 57 (1-2): 20-33. doi:10.1007/s002530100745.

PRO-ACT. 1999. "Petroleum Fuels: Basic Composition and Properties". PRO-ACT. http://infohouse.p2ric.org/ref/07/06026.htm.

Quensen, John F., Stephen A. Boyd, and James M. Tiedje. 1990. "Dechlorination of Four Commercial Polychlorinated Biphenyl Mixtures (Aroclors) by Anaerobic Microorganisms from Sediments." Applied and Environmental Microbiology 56 (8): 2360-69.

Quensen, John F., James M. Tiedje, and Stephen A. Boyd. 1988. "Reductive Dechlorination of Polychlorinated Biphenyls by Anaerobic Microorganisms from Sediments." Science 242 (4879): 752+.

Rahman, K. S. M., T. J. Rahman, Y. Kourkoutas, I. Petsas, R. Marchant, and I. M. Banat. 2003. "Enhanced Bioremediation of N-Alkane in Petroleum Sludge Using Bacterial Consortium Amended with Rhamnolipid and Micronutrients." Bioresource Technology 90 (2): 159-68. doi:10.1016/S0960-8524(03)00114-7.

Rehmann, Lars, and Andrew J. Daugulis. 2008. "Bioavailability of PCBs in Biphasic Bioreactors." Biochemical Engineering Journal 38 (2): 219-25. doi:10.1016/j.bej.2007.07.004.

Richardson, Stephen D., Maiysha D. Jones, David R. Singleton, and Michael D. Aitken. 2012. "Long-Term Simulation of in Situ Biostimulation of Polycyclic Aromatic Hydrocarbon-Contaminated Soil." Biodegradation 23 (4): 621-33. doi:10.1007/s10532-012-9538-9. 
Sakaki, Toshiyuki, and Eiji Munetsuna. 2010. "Enzyme Systems for Biodegradation of Polychlorinated Dibenzo-P-Dioxins." Applied Microbiology and Biotechnology 88 (1): 23-30. doi:10.1007/s00253-010-2765-2.

Sarkar, Dibyendu, Michael Ferguson, Rupali Datta, and Stuart Birnbaum. 2005.

"Bioremediation of Petroleum Hydrocarbons in Contaminated Soils: Comparison of Biosolids Addition, Carbon Supplementation, and Monitored Natural Attenuation." Environmental Pollution 136 (1): 187-95. doi:10.1016/j.envpol.2004.09.025.

Seeger, Michael, Kenneth N. Timmis, and Bernd Hofer. 1997. "Bacterial Pathways for the Degradation of Polychlorinated Biphenyls." Marine Chemistry 58 (3-4): 327 33. doi:10.1016/S0304-4203(97)00059-5.

Sigma Aldrich. 2008. "Bushnell Haas Agar Product Information." https://www.sigmaaldrich.com/content/dam/sigmaaldrich/docs/Sigma/Datasheet/b4926dat.pdf.

. 2012. "Primers and Fluorescent Probes For Quantitative Real-Time PCR and Other Applications". Sigma Aldrich. http://www.sigmaaldrich.com/content/dam/sigmaaldrich/docs/Sigma/Brochure/fluorescent_dna_probes.pdf.

Singer, Andrew C., Charles S. Wong, and David E. Crowley. 2002. "Differential Enantioselective Transformation of Atropisomeric Polychlorinated Biphenyls by Multiple Bacterial Strains with Different Inducing Compounds." Applied and Environmental Microbiology 68 (11): 5756-59. doi:10.1128/AEM.68.11.57565759.2002 .

Sinkkonen, Seija, and Jaakko Paasivirta. 2000. "Degradation Half-Life Times of PCDDs, PCDFs and PCBs for Environmental Fate Modeling." Chemosphere 40 (9): 94349.

Srivastava, Smita, Madhulika Singh, Jasmine George, Kulpreet Bhui, Anand Murari Saxena, and Yogeshwer Shukla. 2010. "Genotoxic and Carcinogenic Risks Associated with the Dietary Consumption of Repeatedly Heated Coconut Oil." The British Journal of Nutrition 104 (9): 1343-52. doi:10.1017/S0007114510002229.

SSFL WG. 2014. "About SSFL | Santa Susana Field Laboratory Work Group." http://www.ssflworkgroup.org/about-ssfl/.

Staley, James T., and Anna-Louise Reysenbach. 2002. Biodiversity of Microbial Life: Foundation of Earth's Biosphere. Wiley.

Stobart, Ben, Richard Warwick, Csar Gonzlez, Sandra Mallol, David Daz, Olga Reones, and Raquel Goi. 2009. "Long-Term and Spillover Effects of a Marine Protected 
Area on an Exploited Fish Community." Marine Ecology Progress Series 384 (May): 47-60. doi:10.3354/meps08007.

Suhara, H., C. Daikoku, H. Takata, S. Suzuki, Y. Matsufuji, K. Sakai, and R. Kondo. 2003. "Monitoring of White-Rot Fungus during Bioremediation of Polychlorinated Dioxin-Contaminated Fly Ash." Applied Microbiology and Biotechnology 62 (5-6): 601-7. doi:10.1007/s00253-003-1284-9.

Suhara, Hiroto, Ichiro Kamei, Nitaro Maekawa, and Ryuichiro Kondo. 2011. "Biotransformation of Polychlorinated Dibenzo-P-Dioxin by Coprinellus Species." Mycoscience 52 (1): 48-52. doi:10.1007/s10267-010-0062-7.

Takada, S, M Nakamura, T Matsueda, R Kondo, and K Sakai. 1996a. "Degradation of Polychlorinated Dibenzo-P-Dioxins and Polychlorinated Dibenzofurans by the White Rot Fungus Phanerochaete Sordida YK-624." Applied and Environmental Microbiology 62 (12): 4323-28.

1996b. "Degradation of Polychlorinated Dibenzo-P-Dioxins and Polychlorinated Dibenzofurans by the White Rot Fungus Phanerochaete Sordida YK-624." Applied and Environmental Microbiology 62 (12): 4323-28.

Thirukkumaran, Chandini M, and Dennis Parkinson. 2000. "Microbial Respiration, Biomass, Metabolic Quotient and Litter Decomposition in a Lodgepole Pine Forest Floor Amended with Nitrogen and Phosphorous Fertilizers." Soil Biology and Biochemistry 32 (1): 59-66. doi:10.1016/S0038-0717(99)00129-7.

Thomas, Dr, Ks Carswell, and G. Georgiou. 1992. "Mineralization of Biphenyl and Pcbs by the White Rot Fungus Phanerochaete-Chrysosporium." Biotechnology and Bioengineering 40 (11): 1395-1402. doi:10.1002/bit.260401114.

Thomas, Valerie M., and Thomas G Sprio. 1994. "An Estimation of Dioxin Emissions in the United States." Toxicological and Environmental Chemistry 50 (December): $1-37$.

Tiedje, James M., John F. Quensen Iii, Joann Chee-Sanford, Joshua P. Schimel, and Stephen A. Boyd. 1993. "Microbial Reductive Dechlorination of PCBs." Biodegradation 4 (4): 231-40. doi:10.1007/BF00695971.

Tiedje, JM, JF III Quensen, WW Mohn, Joshua P. Schimel, JA cole, and Stephen A. Boyd. 1991. Rossmoore RW (Ed) Biodeterioration and Biodegradation. 8th ed. New York, NY: Elsevier Applied Science,.

Tigini, Valeria, Valeria Prigione, Sara Di Toro, Fabio Fava, and Giovanna C Varese. 2009. "Isolation and Characterisation of Polychlorinated Biphenyl (PCB) Degrading Fungi from a Historically Contaminated Soil." Microbial Cell Factories 8 (January): 5. doi:10.1186/1475-2859-8-5. 
Torlapati, Jagadish, and Michel C. Boufadel. 2014. "Evaluation of the Biodegradation of Alaska North Slope Oil in Microcosms Using the Biodegradation Model BIOB." Frontiers in Microbiology 5 (May). doi:10.3389/fmicb.2014.00212.

Tortella, Gonzalo, Maria Diez, and Nelson Durá. 2005. "Fungal Diversity and Use in Decomposition of Environmental Pollutants." Critical Reviews in Microbiology 31 (4): 197-212. doi:10.1080/10408410500304066.

Townsend, G. Todd, Roger C. Prince, and Joseph M. Suflita. 2003. "Anaerobic Oxidation of Crude Oil Hydrocarbons by the Resident Microorganisms of a Contaminated Anoxic Aquifer." Environmental Science \& Technology 37 (22). AMER CHEMICAL SOC: 5213-18. doi:10.1021/es0264495.

Trapp, Stefan, and Michael Matthies. 1997. "Modeling Volatilization of PCDD/F from Soil and Uptake into Vegetation." Environmental Science \& Technology 31 (1). American Chemical Society: 71-74. doi:10.1021/es960133d.

Tř́ska, Jan, Gabriela Kuncová, Martina Macková, Hana Nováková, Jaakko Paasivirta, Mirja Lahtiperä, and Naděžda Vrchotová. 2004. "Isolation and Identification of Intermediates from Biodegradation of Low Chlorinated Biphenyls (Delor-103)." Chemosphere 54 (6): 725-33. doi:10.1016/j.chemosphere.2003.08.021.

Tuppurainen, Kari, Arja Asikainen, Päivi Ruokojärvi, and Juhani Ruuskanen. 2003. "Perspectives on the Formation of Polychlorinated Dibenzo-P-Dioxins and Dibenzofurans during Municipal Solid Waste (MSW) Incineration and Other Combustion Processes." Accounts of Chemical Research 36 (9): 652-58. doi:10.1021/ar020104+.

Turyk, Mary E, Henry a Anderson, and Victoria W Persky. 2007. "Relationships of Thyroid Hormones with Polychlorinated Biphenyls, Dioxins, Furans, and DDE in Adults." Environmental Health Perspectives 115 (8): 1197-1203. doi:10.1289/ehp.10179.

Tyagi, Meenu, M. Manuela R. da Fonseca, and Carla C. C. R. de Carvalho. 2011a. "Bioaugmentation and Biostimulation Strategies to Improve the Effectiveness of Bioremediation Processes." Biodegradation 22 (2): 231-41. doi:10.1007/s10532010-9394-4.

Tyagi, Meenu, M. Manuela R. da Fonseca, and Carla C. C. R. de Carvalho. 2011 b. "Bioaugmentation and Biostimulation Strategies to Improve the Effectiveness of Bioremediation Processes." Biodegradation 22 (2): 231-41. doi:10.1007/s10532010-9394-4.

U.S. Department of Health and Human Services. 1999. "TOXICOLOGICAL PROFILE FOR TOTAL PETROLEUM HYDROCARBONS ( TPH )", no. September. 
Udvardi, Michael K., Tomasz Czechowski, and Wolf-Rudiger Scheible. 2008. "Eleven Golden Rules of Quantitative RT-PCR.” The Plant Cell 20 (7): 1736-37. doi:10.1105/tpc.108.061143.

Ulrich, Glenn A., and Joseph Suflita. 2001. "Enhancement of Microbial Sulfate Reduction for the Remediation of Hydrocarbon Contaminated Aquifers - a Laboratory and Field-Scale Project - Final Report Executive Summary."

US EPA. 2010. "Hudson River PCBs." US EPA. http://www.epa.gov/hudson/background.htm.

US EPA, OSWER. 2013. "INFORMATION ON OSWER DIRECTIVE 9200.4-17P". Policies \& Guidance. Accessed October 11. http://www.epa.gov/oust/directiv/d9200417.htm.

Valli, K, H Wariishi, and M H Gold. 1992. "Degradation of 2,7-Dichlorodibenzo-PDioxin by the Lignin-Degrading Basidiomycete Phanerochaete Chrysosporium." Journal of Bacteriology 174 (7): 2131-37.

Van Beilen, Jan B, and Enrico G Funhoff. 2007. "Alkane Hydroxylases Involved in Microbial Alkane Degradation." Applied Microbiology and Biotechnology 74 (1): 13-21. doi:10.1007/s00253-006-0748-0.

Van Beilen, JB, WA Duetz, THM Smits, and B Witholt. 2003. "Diversity of Alkane Hydroxylase Systems in the Environment." Oil Gas Science Technology, no. 58: $427-40$.

Van den Berg, M, L Birnbaum, A T Bosveld, B Brunstrom, P Cook, M Feeley, J P Giesy, et al. 1998. "Toxic Equivalency Factors (TEFs) for PCBs, PCDDs, PCDFs for Humans and Wildlife." Environmental Health Perspectives 106 (12): 775-92.

Van den Berg, M, L Birnbaum, a T Bosveld, B Brunström, P Cook, M Feeley, J P Giesy, et al. 1998. "Toxic Equivalency Factors (TEFs) for PCBs, PCDDs, PCDFs for Humans and Wildlife." Environmental Health Perspectives 106 (12): 775-92.

Van Hamme, J. D., A. Singh, and O. P. Ward. 2003. "Recent Advances in Petroleum Microbiology." Microbiology and Molecular Biology Reviews 67 (4): 503-+. doi:10.1128/MMBR.67.4.503-549.2003.

Vasquez, Astrid P., James L. Regens, and James T. Gunter. 2004. "Environmental Persistence of 2,3,7,8-Tetrachlorodibenzo-P-Dioxin in Soil around Hardstand 7 at Eglin Air Force Base, Florida." Journal of Soils and Sediments 4 (3): 151-56. doi:10.1007/BF02991132.

Vilanova, Rosa M., Pilar Fernández, Carolina Martínez, and Joan O. Grimalt. 2001. "Polycyclic Aromatic Hydrocarbons in Remote Mountain Lake Waters." Water Research 35 (16): 3916-26. doi:10.1016/S0043-1354(01)00113-0. 
Wang, Shu-Li, Yu-Chen Chang, How-Ran Chao, Chien-Ming Li, Lih-Ann Li, Long-Yau Lin, and Olaf Päpke. 2006. "Body Burdens of Polychlorinated Dibenzo-PDioxins, Dibenzofurans, and Biphenyls and Their Relations to Estrogen Metabolism in Pregnant Women." Environmental Health Perspectives 114 (5): 740-45. doi:10.1289/ehp.8809.

Whittaker, Martin, and Simon J. T. Pollard. 1997. “A Performance Assessment of Source Correlation and Weathering Indices for Petroleum Hydrocarbons in the Environment." Environmental Toxicology and Chemistry 16 (6): 1149-58. doi:10.1002/etc.5620160609.

WHO. 1992. "Polychlorinated Biphenyls and Polychlorinated Terphenyls (PCBs and PCTs) Health and Saftey Guide \#68." http://www.inchem.org/documents/hsg/hsg/hsg68.htm.

Wiedemeier, Todd H., Hanadi S. Rifai, Charles J. Newell, and John T. Wilson. 1999. Natural Attenuation of Fuels and Chlorinated Solvents in the Subsurface. Wiley.

Wiegel, Juergen, and Qingzhong Wu. 2000. "Microbial Reductive Dehalogenation of Polychlorinated Biphenyls.” FEMS Microbiology Ecology 32 (1): 1-15. doi:10.1111/j.1574-6941.2000.tb00693.x.

Wild, S R, and K C Jones. 1995. "Polynuclear Aromatic Hydrocarbons in the United Kingdom Environment: A Preliminary Source Inventory and Budget." Environmental Pollution (Barking, Essex: 1987) 88 (1): 91-108.

Wilhelm, Jochen, and Alfred Pingoud. 2003. "Real-Time Polymerase Chain Reaction." ChemBioChem 4 (11): 1120-28. doi:10.1002/cbic.200300662.

Wilson, S. C., R. E. Alcock, A. P. Sewart, and K. C. Jones. 1997. "Persistence of Organic Contaminants in Sewage Sludge-Amended Soil: A Field Experiment." Journal of Environmental Quality 26 (6): 1467-77.

Wittich, R-M, C Strömpl, E R B Moore, R Blasco, and K N Timmis. 1999. "Interaction of Sphingomonas and Pseudomonas Strains in the Degradation of Chlorinated Dibenzofurans." Journal of Industrial Microbiology \& Biotechnology 23 (4-5): 353-58. doi:10.1038/sj/jim/2900740.

Wu, Q, D L Bedard, and J Wiegel. 1997a. "Effect of Incubation Temperature on the Route of Microbial Reductive Dechlorination of 2,3,4,6-Tetrachlorobiphenyl in Polychlorinated Biphenyl (PCB)-Contaminated and PCB-Free Freshwater Sediments." Applied and Environmental Microbiology 63 (7): 2836-43. . 1997b. "Temperature Determines the Pattern of Anaerobic Microbial Dechlorination of Aroclor 1260 Primed by 2,3,4,6-Tetrachlorobiphenyl in Woods Pond Sediment." Applied and Environmental Microbiology 63 (12): 4818-25. 
Wu, Q., D. L. Bedard, and J. Wiegel. 1996. "Influence of Incubation Temperature on the Microbial Reductive Dechlorination of 2,3,4,6-Tetrachlorobiphenyl in Two Freshwater Sediments." Applied and Environmental Microbiology 62 (11): 417479.

Yateem, A., M.T. Balba, N. Al-Awadhi, and A.S. El-Nawawy. 1998. "White Rot Fungi and Their Role in Remediating Oil-Contaminated Soil." Environment International 24 (1): 181-87.

Yin, Yuefen, Junhui Guo, Li Zheng, Li Tian, and Xiaoru Wang. 2011. "Capability of Polychlorinated Biophenyl (PCBs) Degrading Fungi Segregated from Sediments." World Journal of Microbiology and Biotechnology 27 (11): 2567-74. doi:10.1007/s11274-011-0728-0.

Yong-lei, An, Lan-ying Zhang, Qiu Ming-ying, Ai-xia Zhou, Zhu Jin-rong, Zhang Lei, and Ren He-jun. 2011. "Screening of PCBs-Degrading Bacteria and Enhanced Bioremediation of Soil under Low-Temperature Condition." In 2011 International Symposium on Water Resource and Environmental Protection (ISWREP), 2:1459-63. doi:10.1109/ISWREP.2011.5893300. 
Appendices

\section{Appendix A: Abbreviations}

AI Atomic International

AOC Administrative Order of Consent

ASTDR Agency for Toxic Substances and Disease Registry

BLAST Basic Local Alignment Search Tool

BTEX Benzene, toluene, ethylbenzene, xylenes

CBP Chlorinated Biphenyl

COI Contaminant of Interest

DD Dibenzodioxin

DF Dibenzofuran

DOD Department of Defense

DOE Department of Energy

DTSC Department of Toxic Substances

EDTA Ethylenediaminetetraacetic acid

EFH Extractable Fuel Hydrocarbons

EPA Enviromental Protection Agency

ETEC Energy Technology Engineering Center 


$\begin{array}{ll}\mathrm{F} & \text { Field } \\ \mathrm{kg} & \text { kilogram } \\ \mathrm{K}_{\text {ow }} & \text { Octanol Water Partition Coefficient } \\ \mathrm{L} & \text { Lab } \\ \mathrm{LiP} & \text { Lignin Peroxidase } \\ \mathrm{MCDF} & \text { Monochlorinated Dibenzofurans } \\ \mathrm{mg} & \text { milligram } \\ \mathrm{mm} & \text { millimeters } \\ \mathrm{MDS} & \text { multidimensional scaling } \\ \mathrm{MnP} & \text { Manganese-dependent Peroxidase }\end{array}$

MTBE Methyl Tertiary Butyl Ether

NAA North American Aviation

NASA National Aeronautics and Space Administration

NCBI National Center for Biotechnology Information

NS Not Stated

NSD Not Significantly Different

PAH Polyaromatic Hydrocarbon 
PCB Polychlorinated Biphenyl
PHC Petroleum Hydrocarbon

PCDD Polychlorinated Diobenzodioxin

PCDFs Polychlorinated Dibenzofurans

pg picograms

ppb parts per billion

ppm parts per million

ppt parts per trillion

RMHF Radioactive Materials Handling Facility

SDS sodium dodecyl sulfate

SSFL Santa Susana Field Laboratory

STIG Soil Treatability Investigation Group

TBA Tetra Butyl Alcohol TBA

TCDD Tetrachloro Dibenzodioxin

TeCDD Tetrachloro Dibenzodioxin

TEF Toxic Equivalency Factor

TEQ Toxic Equivalents 
TPH Total Petroleum Hydrocarbon

ug micrograms

USAF United States Air Force

USEPA United States Environmental Protection Agency

UV Ultra Violet

V Volts 
Appendix B: Additional MDS Scatter Plots

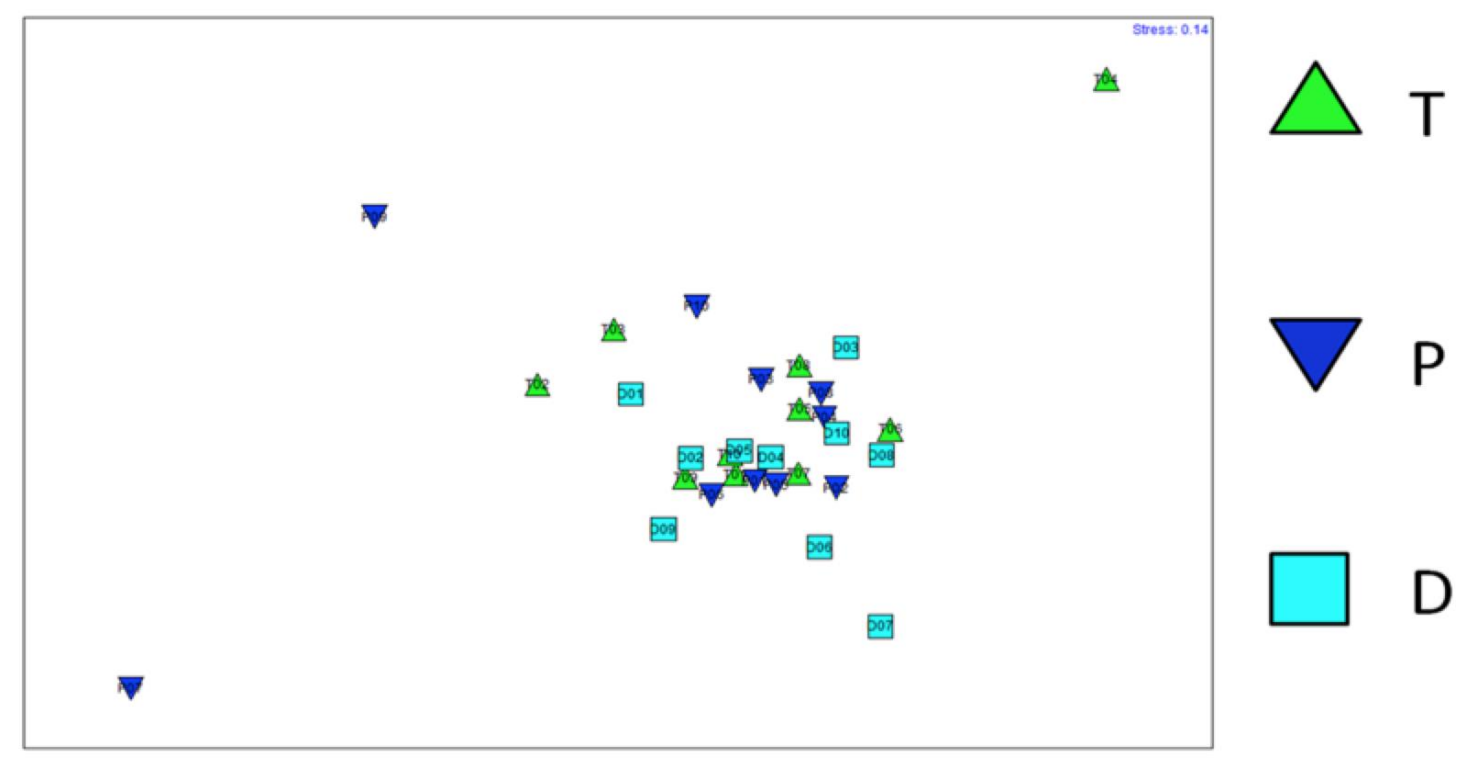

Figure B.1: MDS from bacterial fragments using COI series as a factor

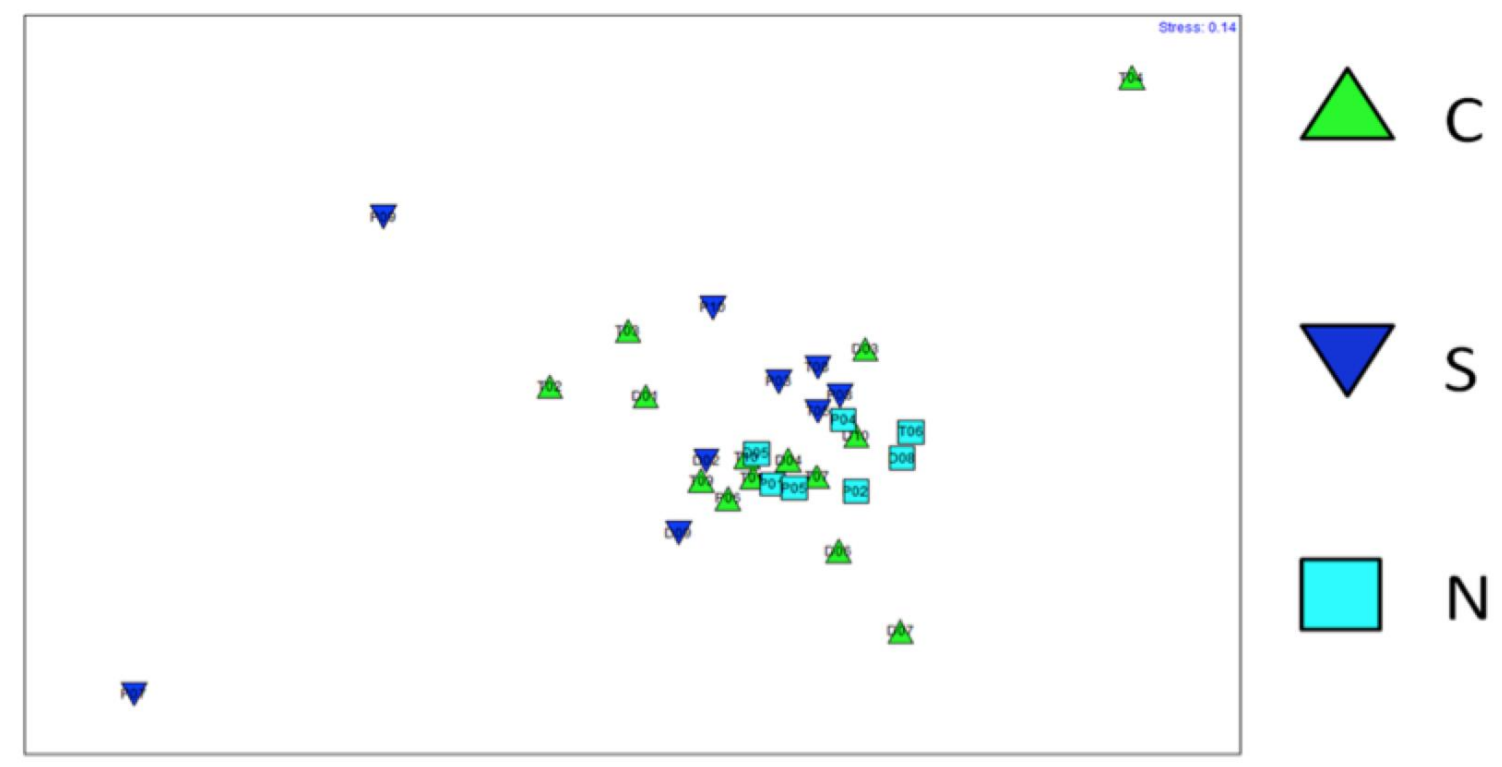

Figure B.2: MDS from bacterial fragments with added factor for location based on the map in Figure 5.1. Sites were labeled them generally south, central, and north. 


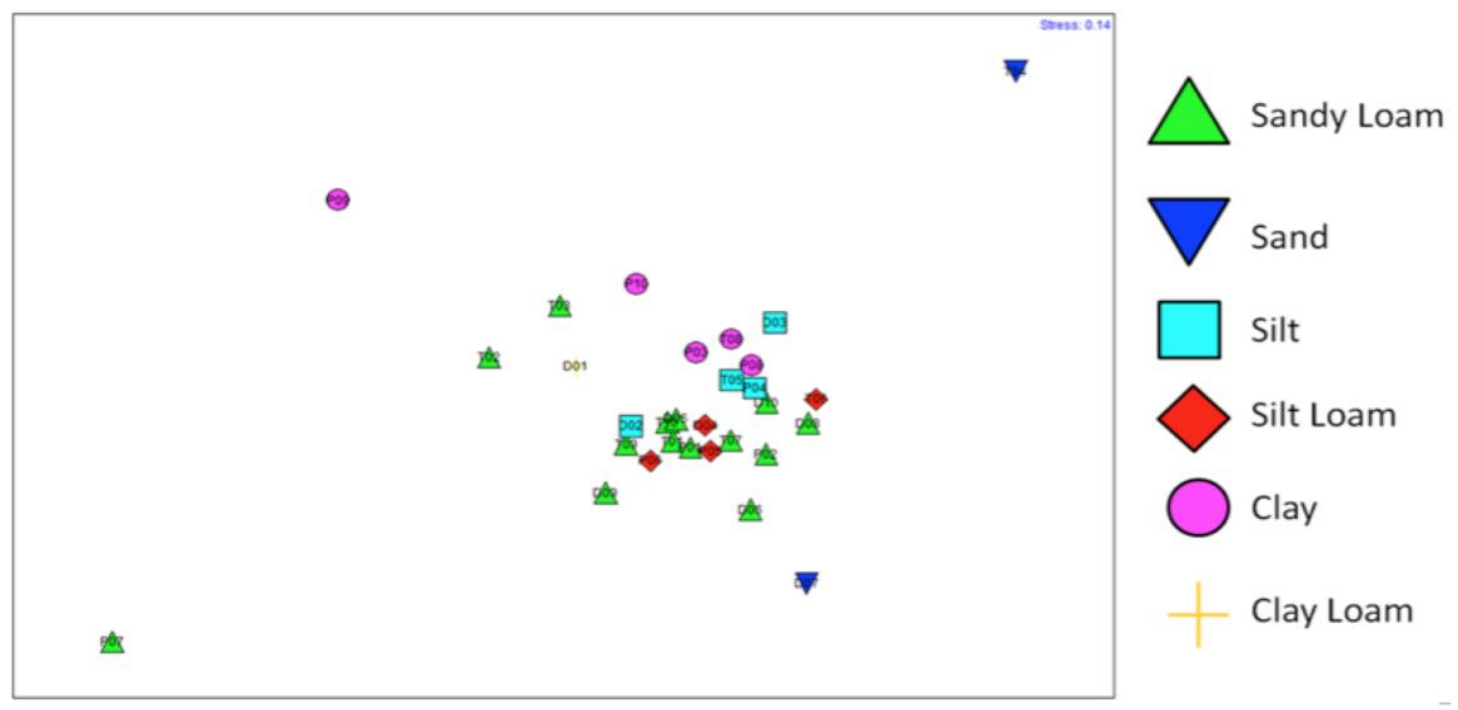

Figure B.3: MDS from bacterial fragments with soil types based on visual observations:

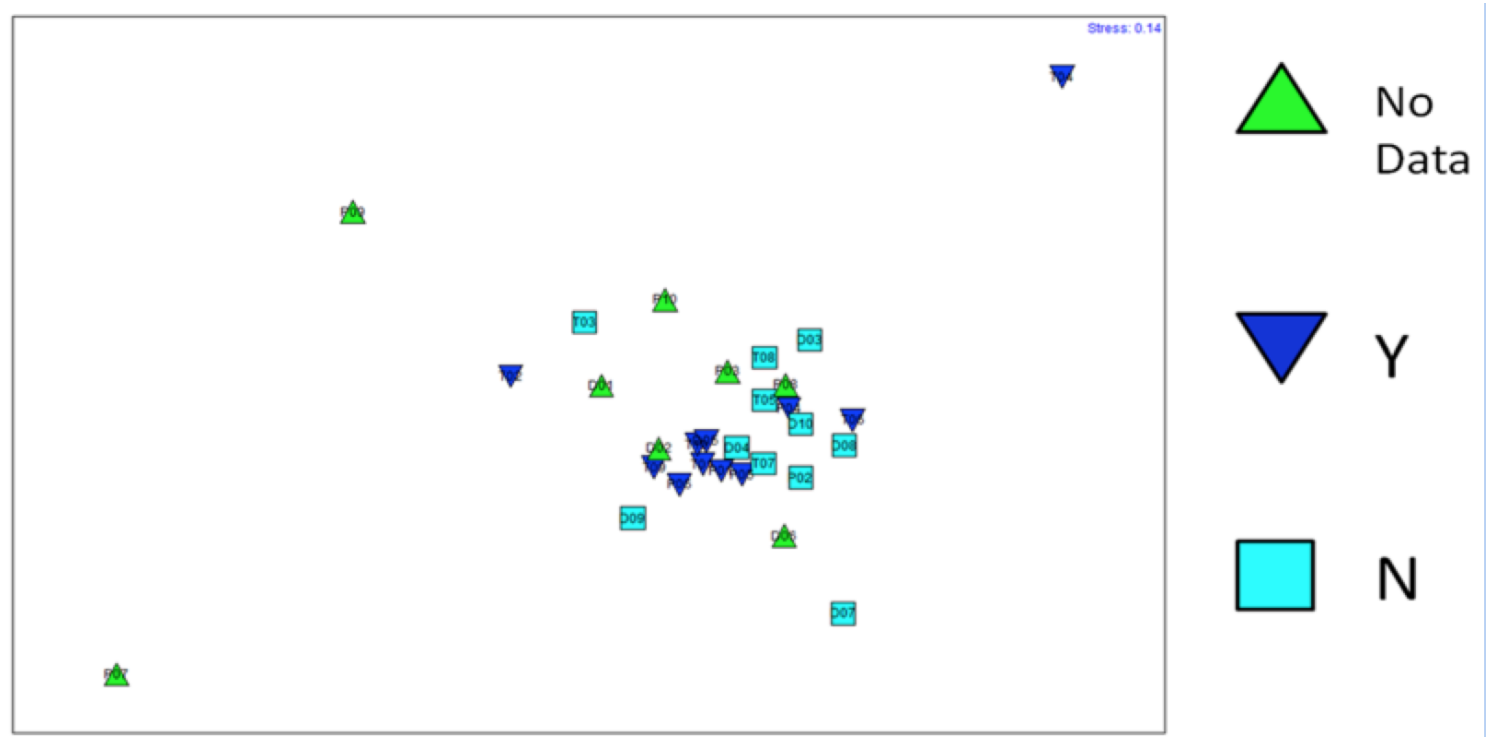

Figure B.4: MDS from bacterial fragments using presences/absence of TPH based on a threshold of $300 \mathrm{ppm}$. 


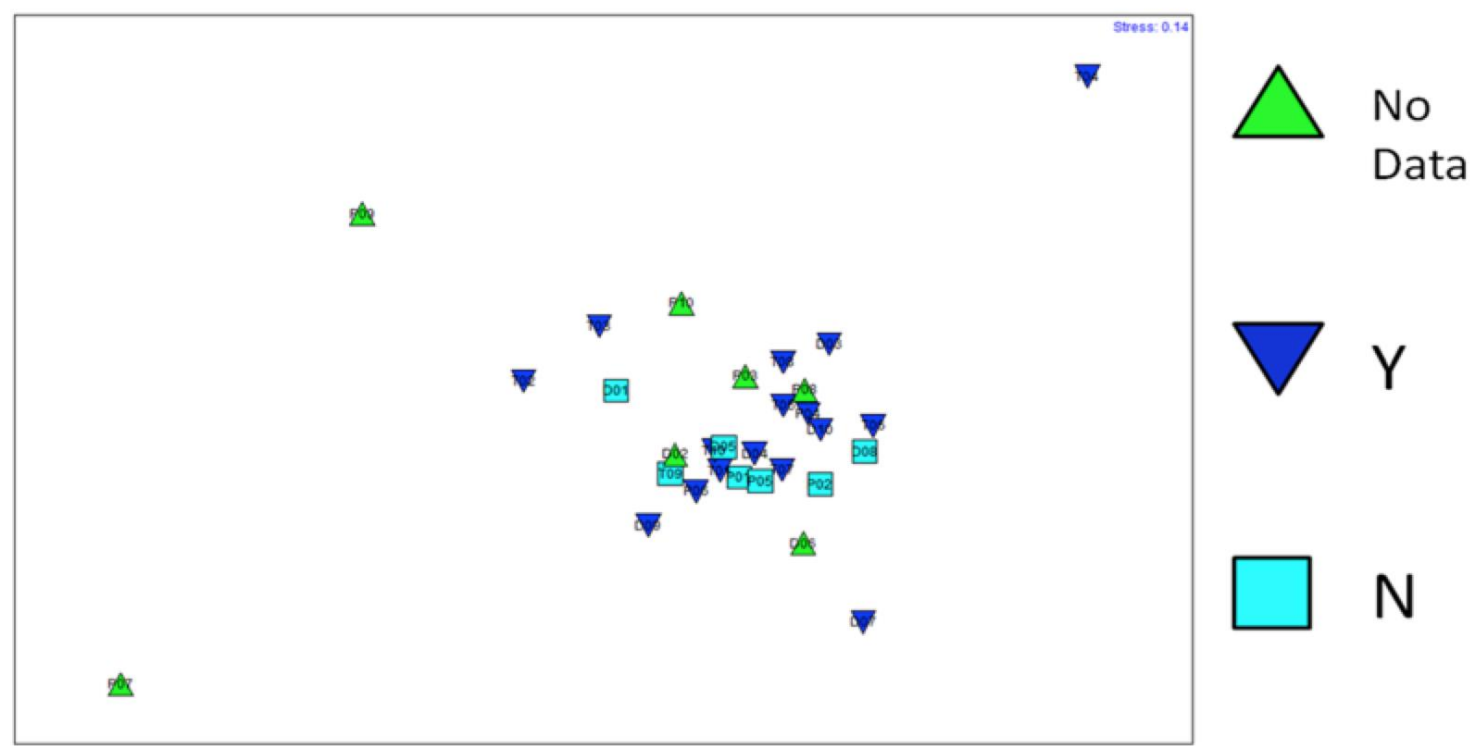

Figure B.5: MDS from bacterial fragments using presences/absence of PAHs based on a threshold of $2.5 \mathrm{ppm}$.

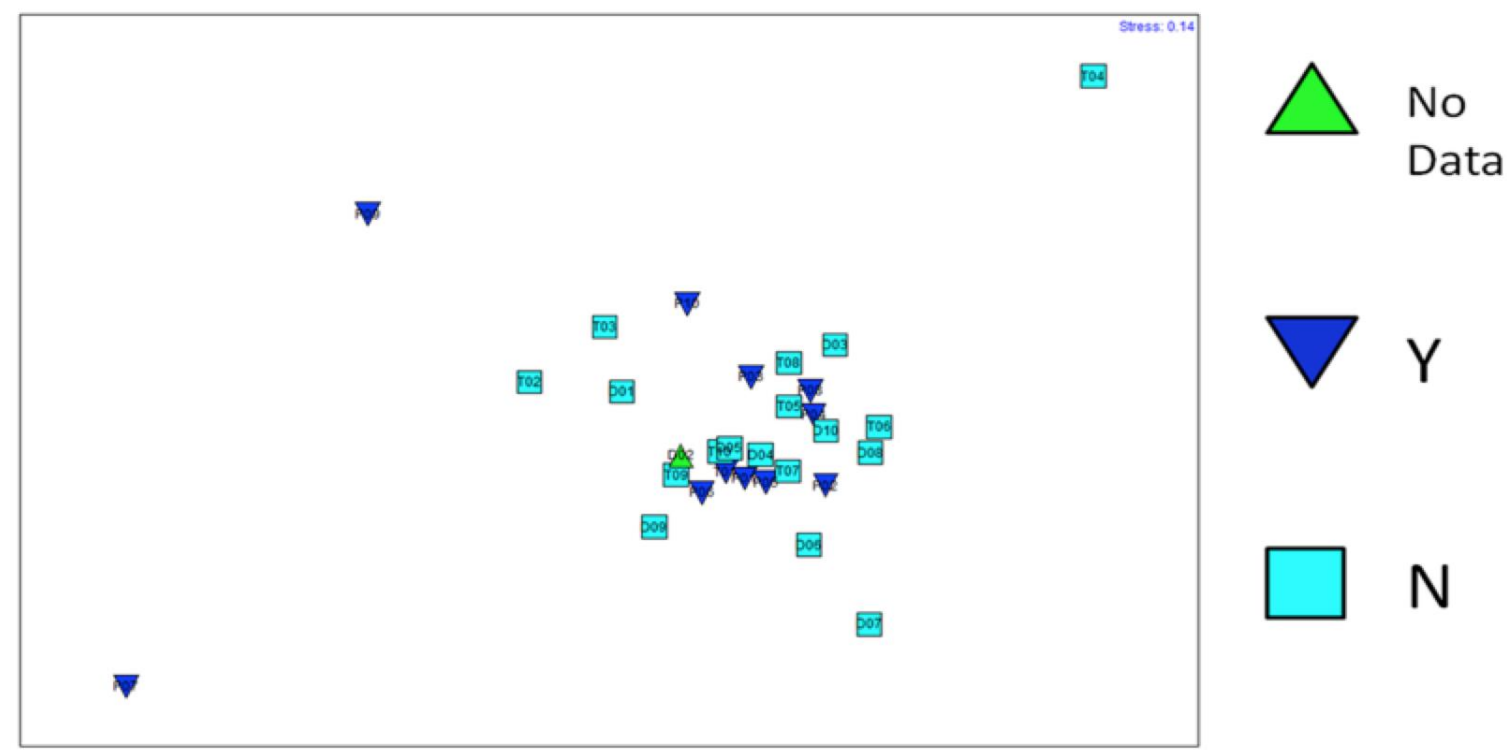

Figure B.6: MDS from bacterial fragments using presences/absence of PCBs based on a threshold of 450 ppb. 


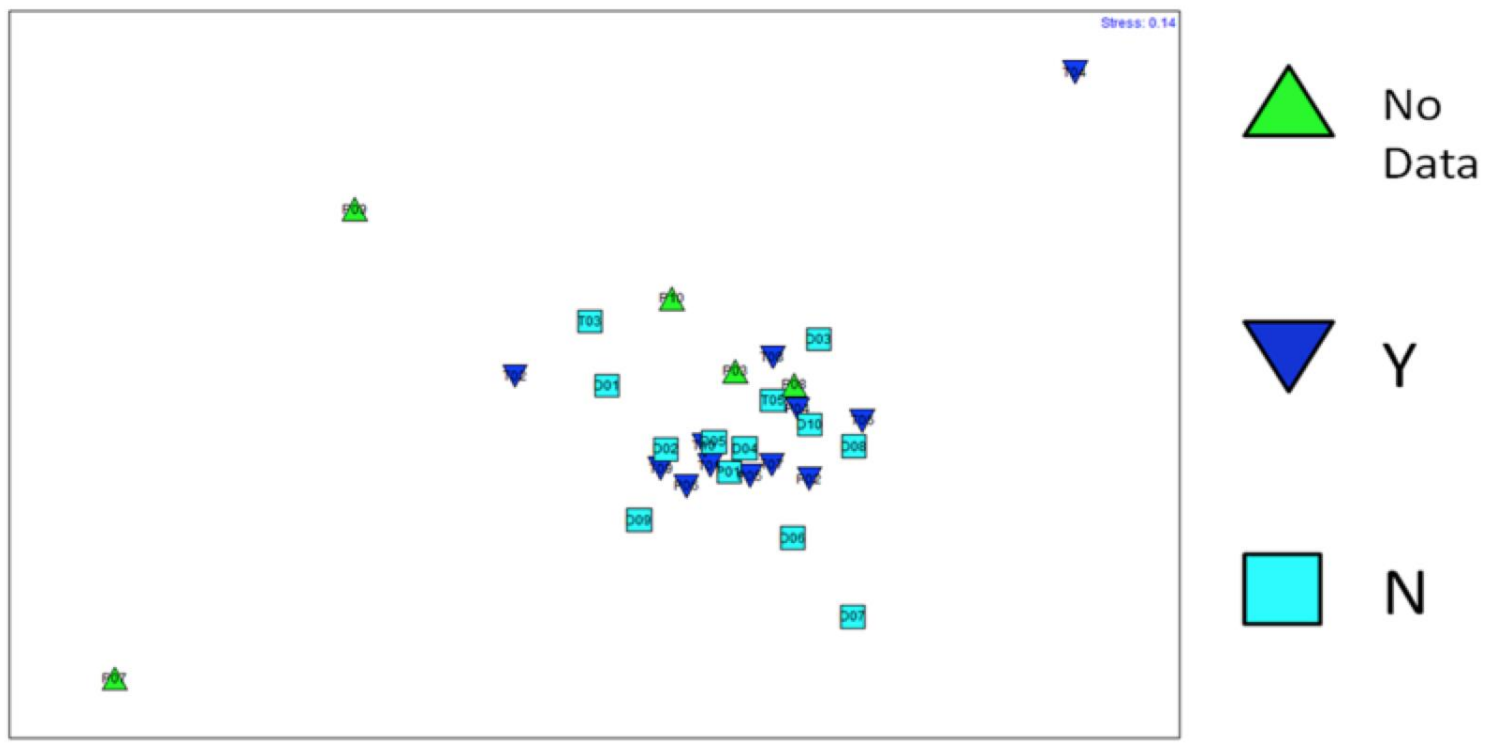

Figure B.7: MDS from bacterial fragments using presences/absence of Dioxin based on a threshold of 5 ppb.

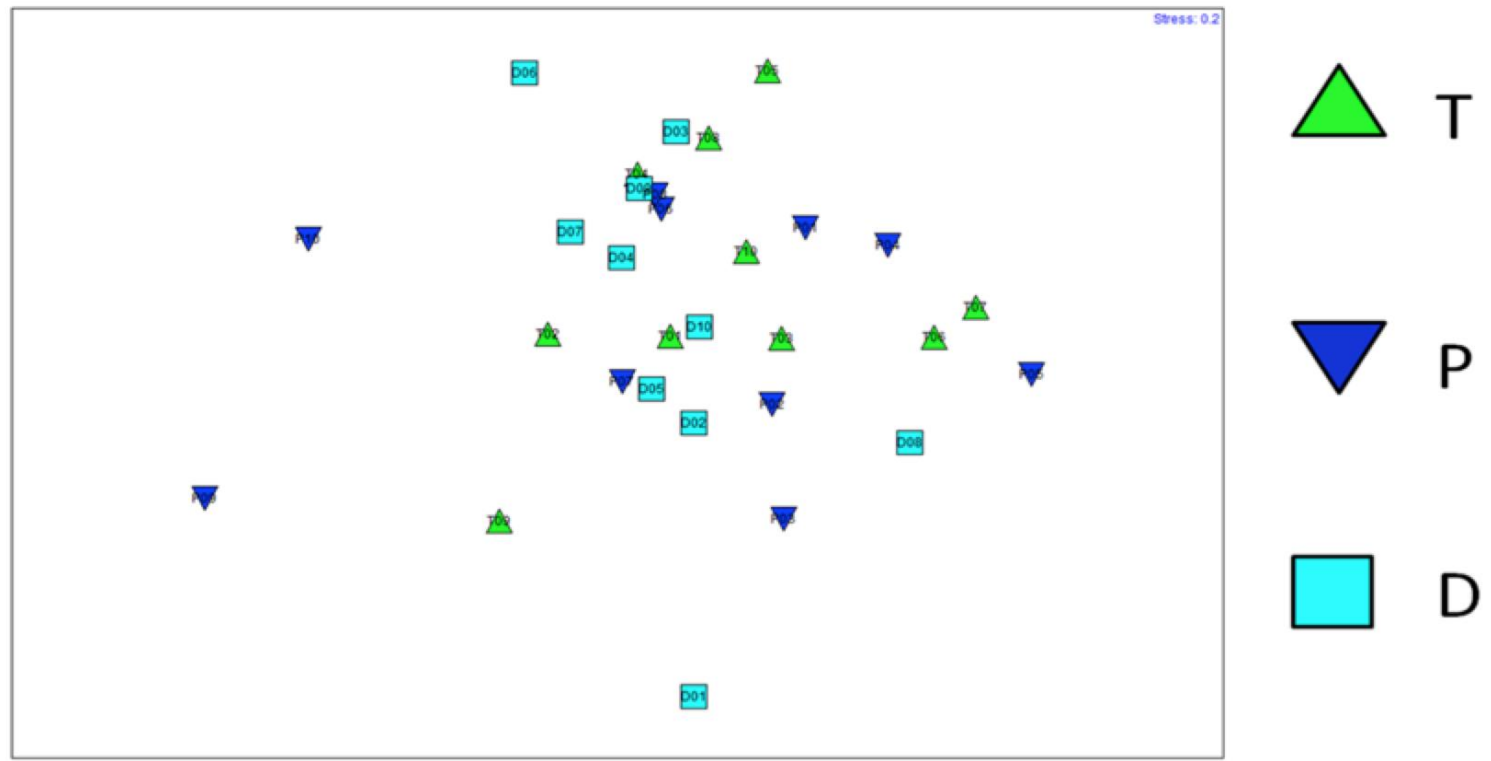

Figure B.8: MDS from fungal fragments using COI series as a factor. 


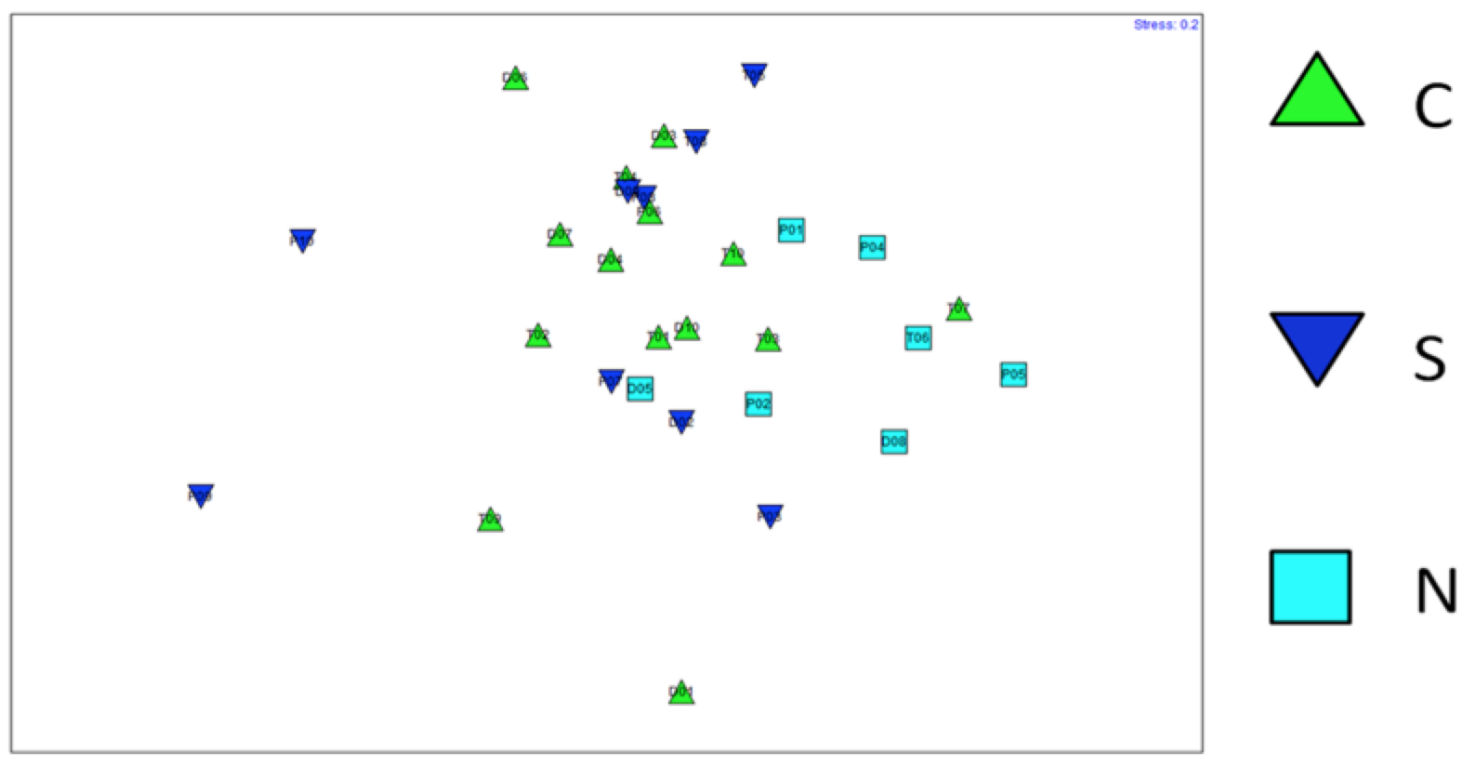

Figure B.9: MDS from fungal fragments with added factor for location based on the map in Figure 5.1. Sites were labeled them generally south, central, and north.

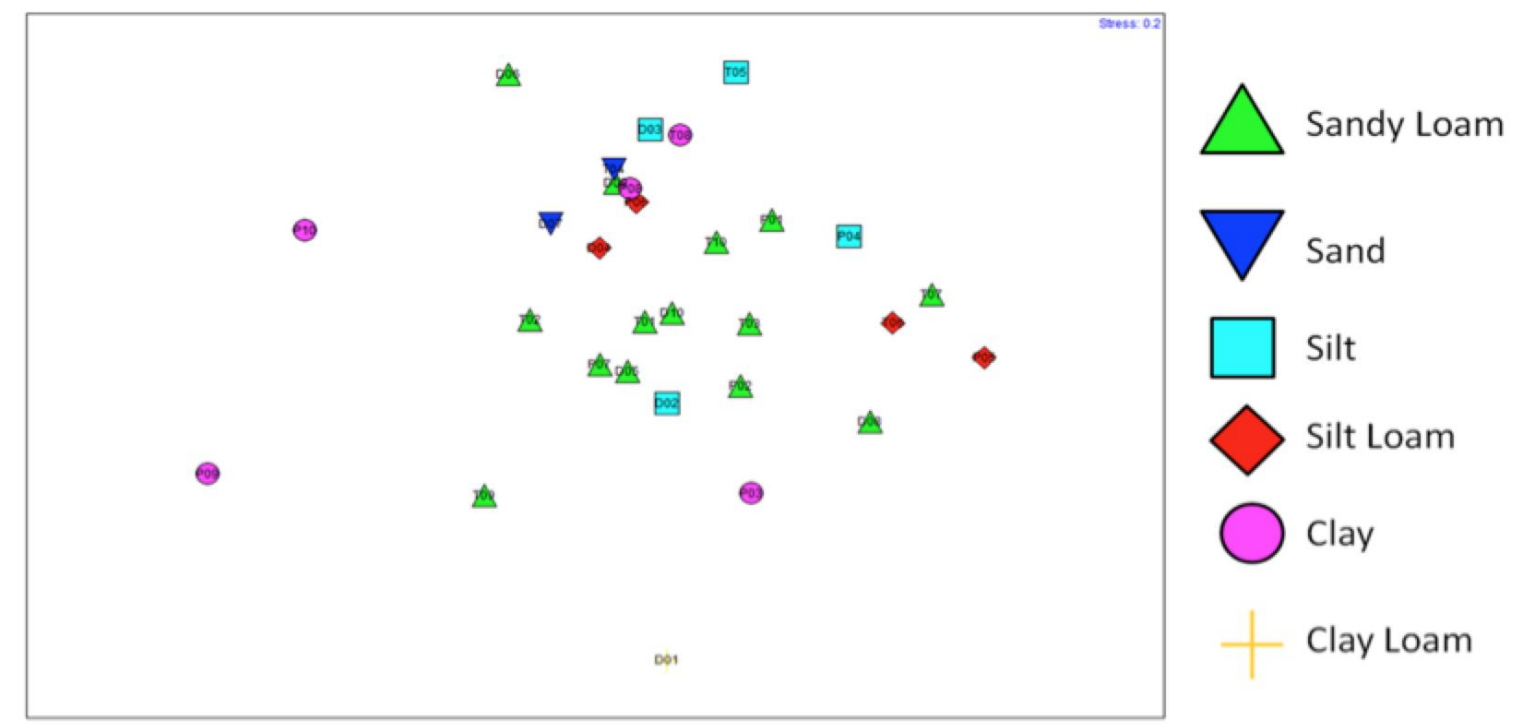

Figure B.10: MDS from fungal fragments with soil types based on visual observations 


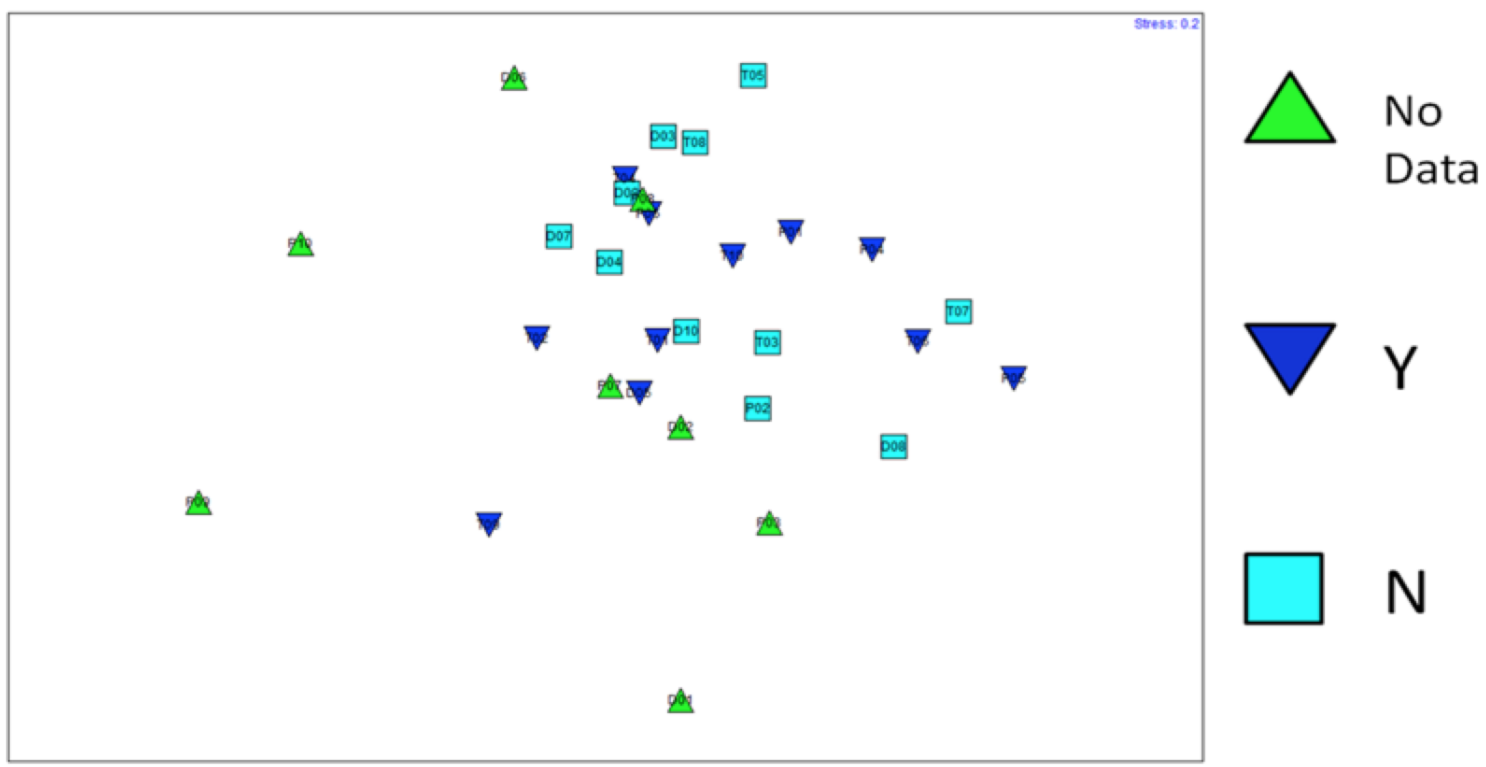

Figure B.11: MDS from fungal fragments using presences/absence of TPH based on a threshold of 300 ppm.

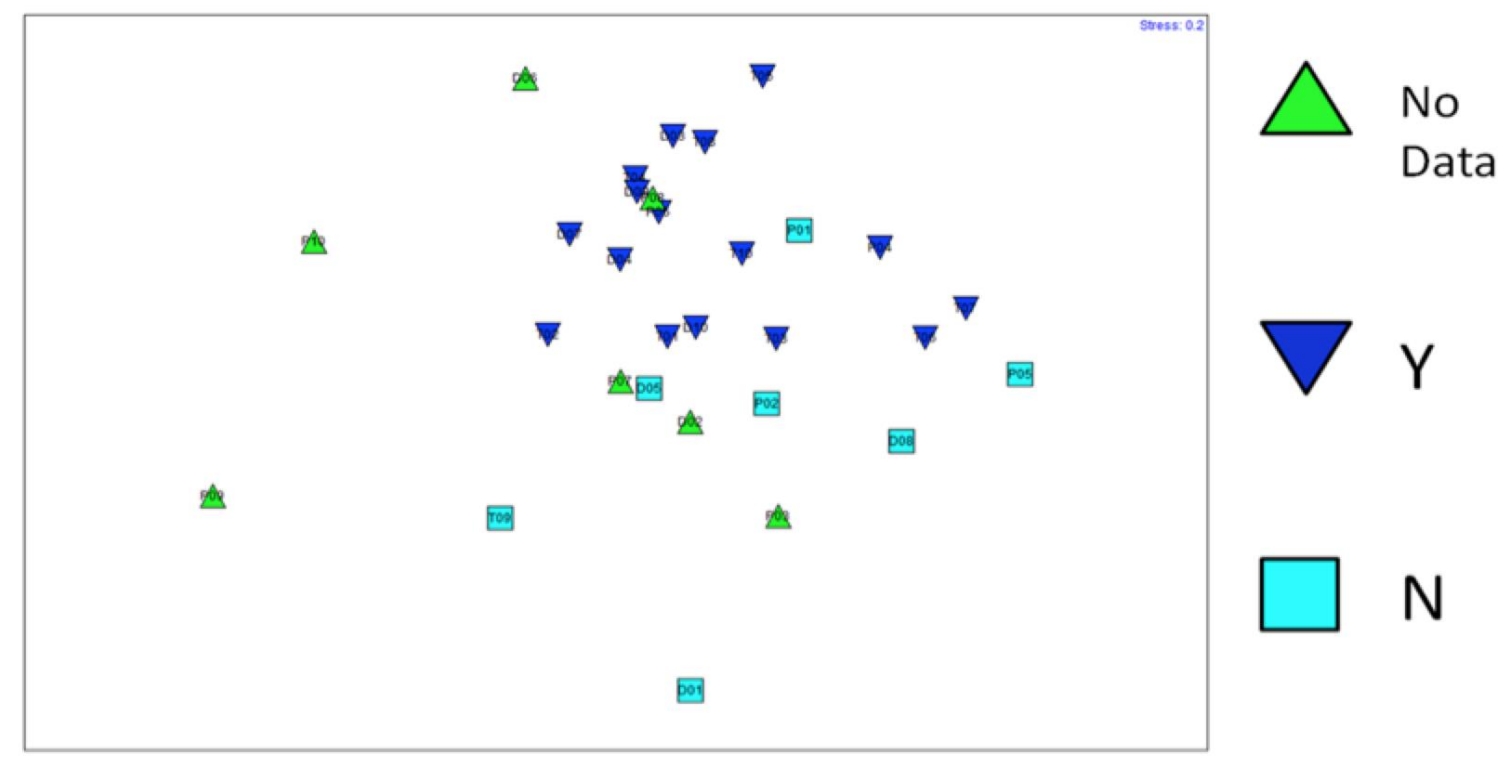

Figure B.12: MDS from fungal fragments using presences/absence of PAHs based on a threshold of $2.5 \mathrm{ppm}$. 


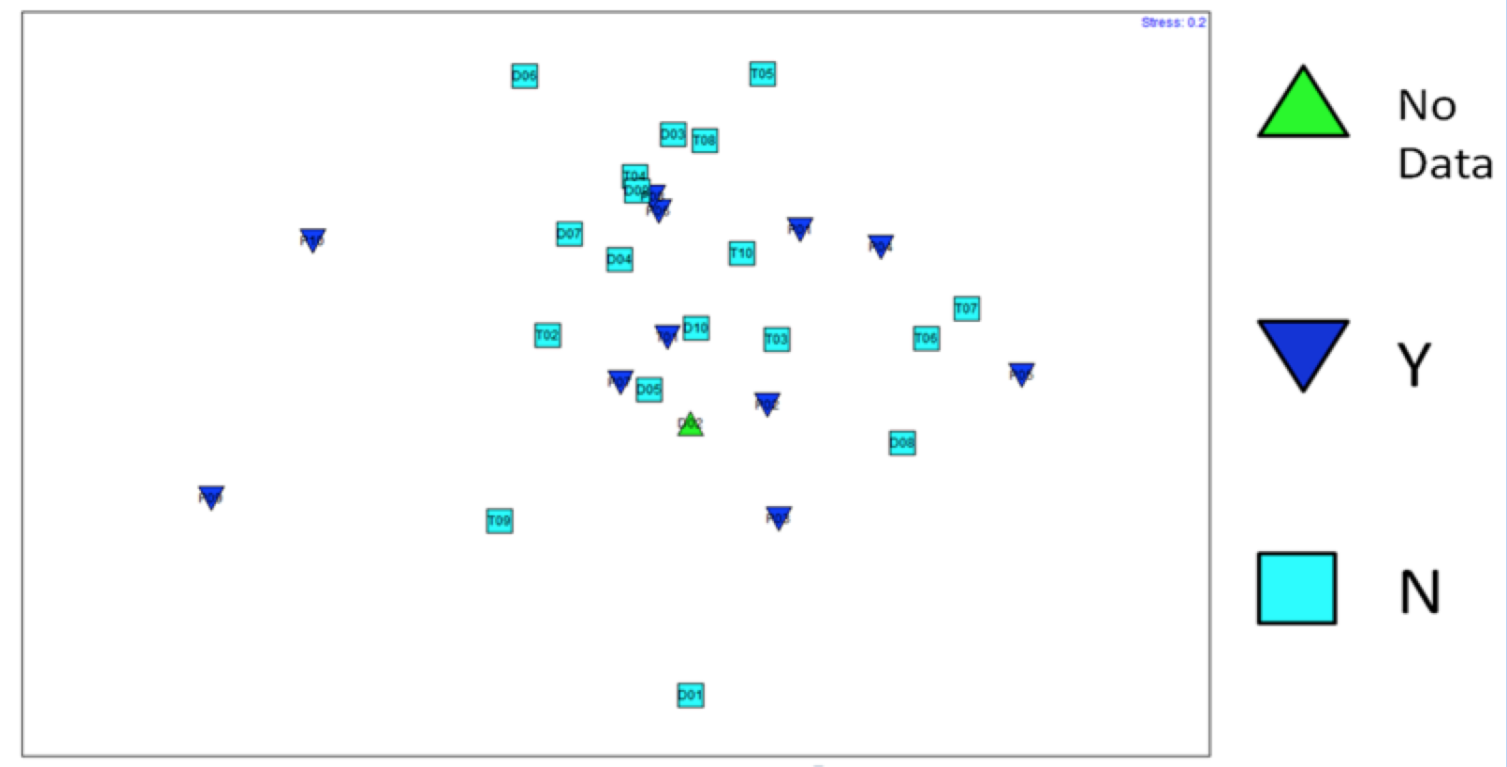

Figure B.13: MDS from fungal fragments using presences/absence of PCBs based on a threshold of $450 \mathrm{ppb}$.

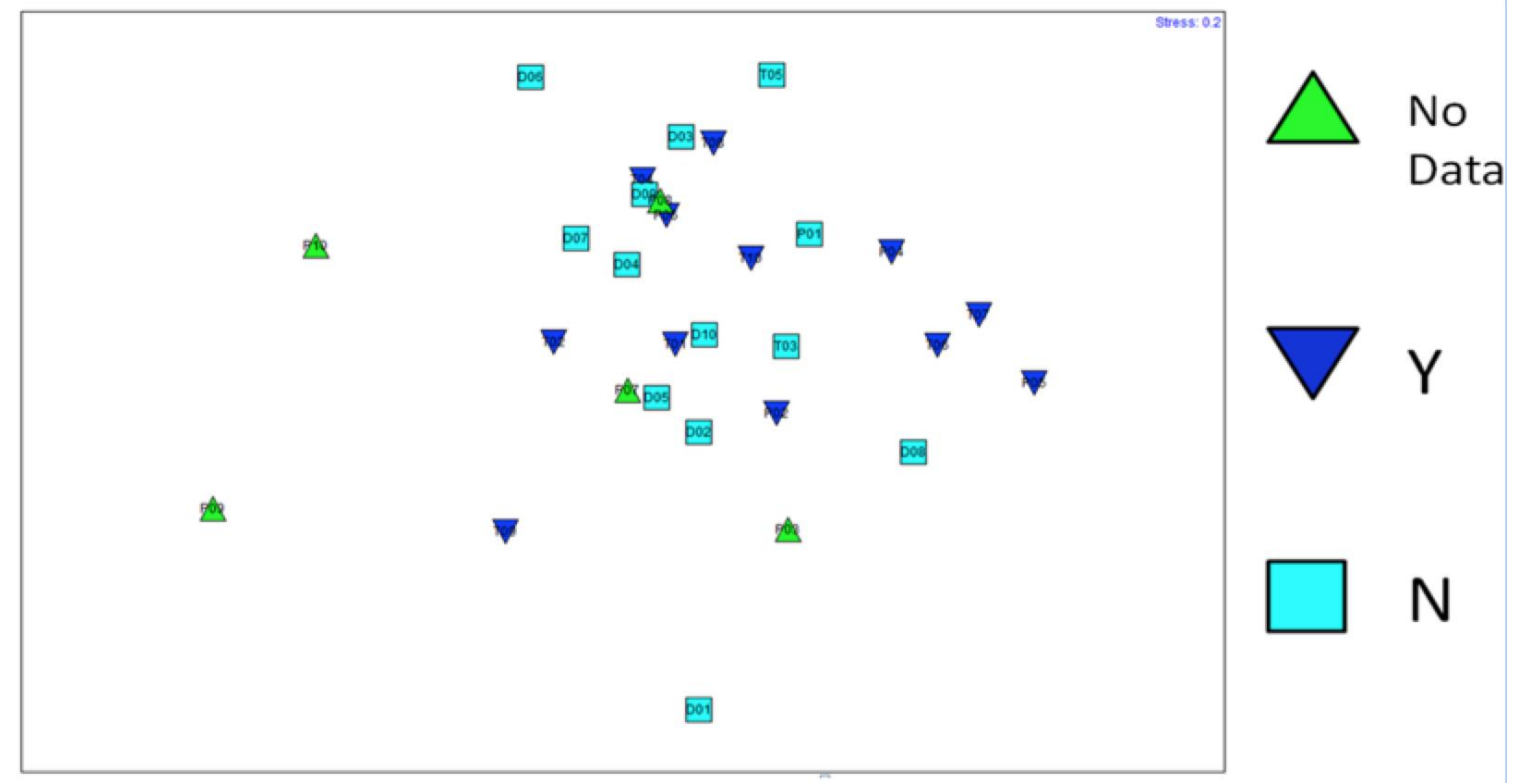

Figure B.14: MDS from fungal fragments using presences/absence of Dioxin based on a threshold of 5 ppb. 